\author{
by \\ Victoria Emily Barbosa Hipolito \\ B.Sc., Biology, Ryerson University, 2014
}

\author{
A dissertation \\ presented at Ryerson University \\ in partial fulfillment of the \\ requirements for the degree of \\ Doctor of Philosophy \\ in the program of \\ Molecular Science
}

Toronto, Ontario, Canada, 2019

(C) Victoria E. B. Hipolito, 2019 


\section{AUTHOR'S DECLARATION FOR ELECTRONIC SUBMISSION OF A DISSERTATION}

I hereby declare that I am the sole author of this dissertation. This is a true copy of the dissertation, including any required final revisions, as accepted by my examiners.

I authorize Ryerson University to lend this dissertation to other institutions or individuals for the purpose of scholarly research

I further authorize Ryerson University to reproduce this dissertation by photocopying or by other means, in total or in part, at the request of other institutions or individuals for the purpose of scholarly research.

I understand that my dissertation may be made electronically available to the public. 


\title{
MECHANISMS OF ORGANELLE IDENTITY AND ADAPTATION IN IMMUNE CELLS
}

\author{
Victoria E. B. Hipolito \\ Doctor of Philosophy, 2019 \\ Molecular Science, Ryerson University
}

\begin{abstract}
Cells are exposed to diverse extracellular and intracellular cues, and coopt subcellular responses depending on their cellular state and functional demand; including upregulating signalling pathways or adapting organelle function and physiology. The immune system is a tightly regulated cohort of specialized cells with heterogeneous functions. Phagocytes, a type of immune cell, are challenged with disparate environmental stimuli and can adapt intracellularly to promote immunity. Due to their cellular plasticity, we aim to understand the molecular machinery that controls organelle identity and adaptation in immune cells, when challenged with immunostimulatory agents.
\end{abstract}

First, we used a long tubular phagocytic cup, which provides the spatiotemporal resolution necessary to study the stages of phagocytosis. Using this model, we observed the sequential recruitment of early and late endolysosomal markers to the growing cup. Surprisingly, the early endosomal lipid, phosphatidylinositol-3-phosphate [PtdIns(3)P] persisted. We determined a novel pH-based mechanism that induces the dissociation of the Vps34 Class III phosphatidylinositol-3kinase from tubular cups as they progressively acidify, when reaching $20 \mu \mathrm{m}$ in length or upon phagosome closure. The detachment of Vps34 stops the production of $\operatorname{PtdIns(3)P,~allowing~for~its~}$ turnover by PIKfyve. Given that PtdIns(3)P dependent signalling is important for multiple cellular pathways, this mechanism for $\mathrm{pH}-$ dependent regulation of $\mathrm{Vps} 34$ could be at the center of many PtdIns(3)P-dependent cellular processes. 
Additionally, we examined how lysosomes, a kingpin organelle essential for pathogen killing, and antigen processing and presentation, adapt in response to phagocyte activation. During phagocyte activation, lysosomes are remodelled from dozens of globular structures to a tubular network, in a process that requires the PI3K-AKT-mTOR signalling pathway. We showed that lysosome tubulation is coupled with an increase in volume and holding capacity. Lysosome remodelling was dependent on de novo synthesis of lysosomal proteins, but independent of TFEB and TFE3 transcription factors, known to scale-up lysosome biogenesis. We demonstrate a novel mechanism of acute organelle expansion via mTORC1-S6K-4E-BP-dependent increase in lysosomal mRNA translation. This process was necessary for efficient and rapid antigen presentation to T-cells by dendritic cells (DCs). Moreover, lysosome remodelling was conserved in DCs activated with select adjuvants, additives used in vaccines to boost efficacy, providing evidence for its possible clinical applicability.

Together, we have identified two novel mechanisms controlling organelle identity and adaptation in immune cells. 


\section{Lay Abstract}

Our immune system is an exquisitely organized system made-up of specialized cells and regulatory networks that respond to the environment to promote immune-health and to protect us from infection. When immune cells recognize an invader, such as a molecular signal derived from pathogenic bacteria, a cascade of cellular and molecular events are initiated to fight the infection. Upon invader-recognition, every stage of the activated subcellular pathway is coordinated by the action of protein and lipid mediators that act as 'guards and check-points' to ensure that each proceeds normally. Understanding how these mediators work in healthy immune cells, can help us better understand what happens in unhealthy immune systems. One pathway that is activated in specialized immune cells called phagocytes, is phagocytosis. During phagocytosis, the cell 'eats' the invading particle whereby it's ingested and then processed within the cell's digestive pathway. Ultimately, the invading particle is degraded inside the stomach-like organelle called the lysosome.

We identified a novel pathway where the acidic environment of the phagosome, the invader-containing compartment, acts like a switch to regulate the activity of key lipid 'guards' that promote particle digestion. Additionally, we also identified a novel pathway within immune cells where lysosomes adapt in size and shape to increase in volume and enhance holding capacity. We determined that this adaptation increases the immune cell's capacity to take up more invading particles and promote T-cell immunity. Together, these two pathways provide us with novel insights into the foundational biology of immune cells that are challenged with invading particles, which may help us better understand how dysregulated immune systems lead to disease, or how dangerous pathogens may exploit these pathways to survive. 


\section{Acknowledgements}

First and foremost, I would like to thank my supervisor and mentor, Dr. Roberto Botelho. Rob, you entrusted me with several opportunities that have and continue to support my professional and personal growth. Thank you for always believing in me and supporting me when it became difficult. Your advice and words of wisdom will continue to motivate me. Additionally, I would like to thank Dr. Costin Antonescu. Both you and Rob have continuously supported me throughout my endeavours in and outside of research, I am forever grateful.

I wish to thank my research and transfer committee members Drs. Antonescu, Fillingham and $\mathrm{McPhee}$ for your valuable feedback and research advice. I appreciate all my collaborators who afforded me the opportunity to develop my research and professional skills when working on their projects. I would especially like to thank Dr. Terebiznik and Amriya Naufer, for inviting me on the PtdIns(3)P-story, which challenged me early-on in my career and prepared me for the highs and lows of research, but, most importantly taught me the true meaning of perseverance.

Importantly, I would like to thank all members of the Botelho lab, past and present, undergrad to post-doc, who have contributed directly or indirectly to my research and time in grad school. With special thanks to those who trained and mentored a novice scientist with no cell biology experience: Drs. Amra Saric, Christopher Choy, Monica Dayam and Matthew Gray.

Thank you to the "backpack club" - AJ, Jee in, Nakita and Tamara, for making my time in MolSci and Ryerson so enjoyable. I am grateful for my MolSci family who have been there with me through all the highs and lows of grad school: Stefanie, Farnaz, Christopher, Stephen, Nora, Yasmin, Monica, ... I can’t wait to see where life brings us all! I truly cherish our friendship.

Special thanks to the "Dream Three" aka "Team tubules", Jacqueline Diaz and Neha Chauhan. As undergrad students, you tackled every new experiment and challenge head-on, your determination and loyalty to me and this project meant so much. And your friendship means more, thanks for keeping me young.

To my close friends AJ, Homai and Chardee, thank you for sticking by me when I seemingly disappeared for months at a time and for the emotional support I needed to complete grad school. Special thanks to my Mom, Dad, Sister and Brother for their endless love and support. And to my partner, Sami, thank you for supporting me through literally everything. 


\section{Table of contents}

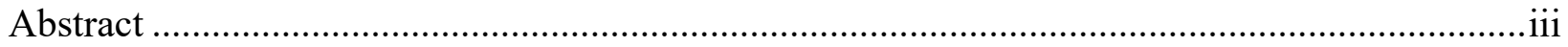

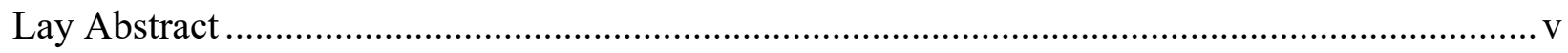

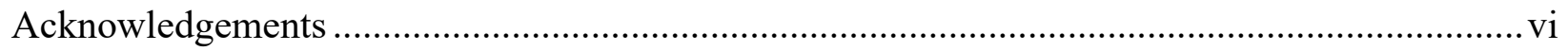

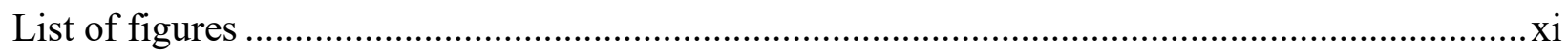

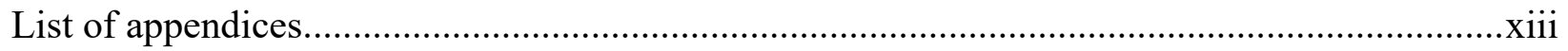

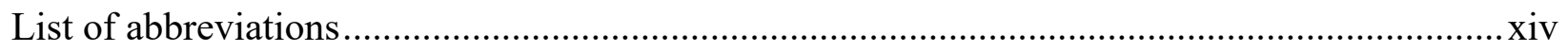

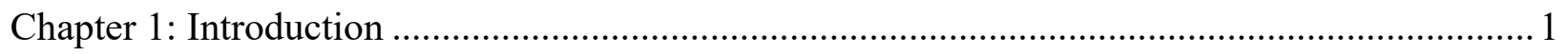

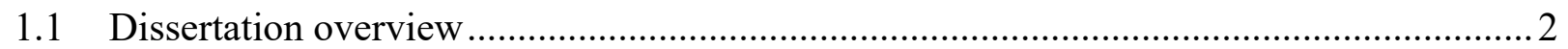

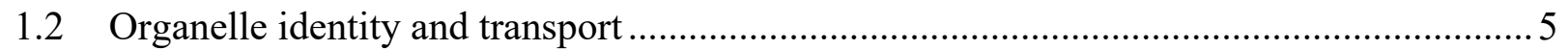

1.2.1 Membrane trafficking through the endo-lysosomal system ..................................5

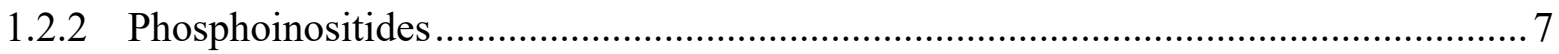

1.2.3 Small GTPases and their effectors ................................................................ 10

1.2.4 Subcellular response to intrinsic and extrinsic stimuli ....................................... 12

1.3 Mammalian Target of Rapamycin nutrient sensing hub .......................................... 14

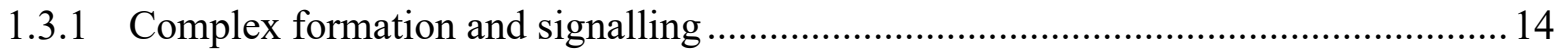

1.3.2 mTORC1 promotes cell growth and proliferation .............................................. 16

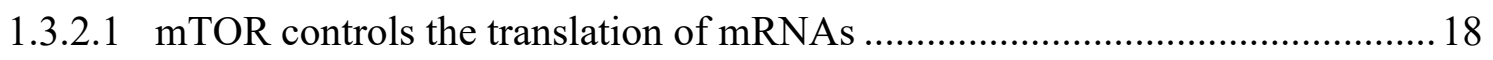

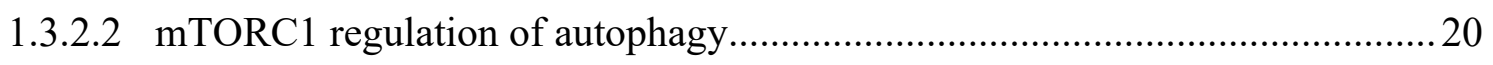

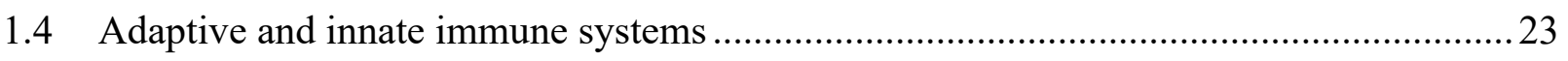

1.4.1 Dendritic cells and macrophages in innate immunity .......................................25

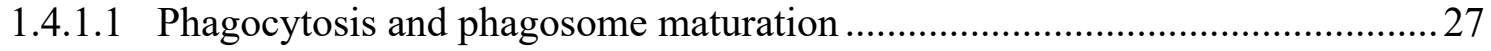

1.4.2 Differentially shaped targets of phagocytosis ....................................................29

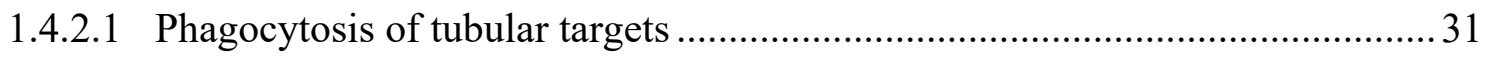

1.4.2.2 Phagocytosis of filamentous Legionella pneumophilia ..................................32

1.4.3 Antigen presentation and processing................................................................... 33

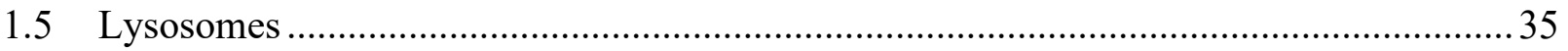

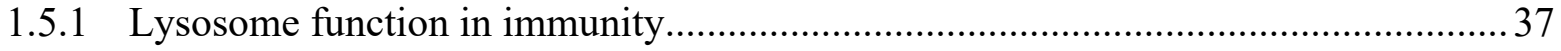

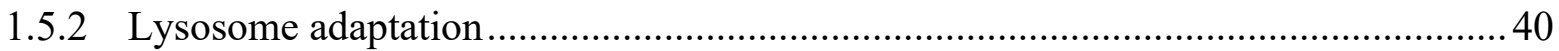

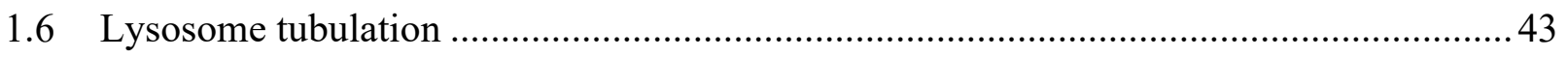

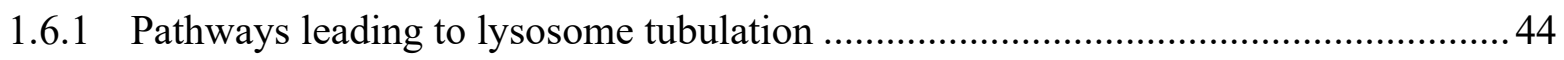


1.6.2 Motors and molecular machinery driving lysosome tubulation............................. 45

1.6.3 Functional implications of lysosome tubulation .......................................... 47

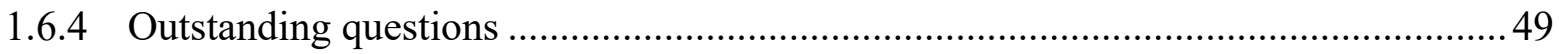

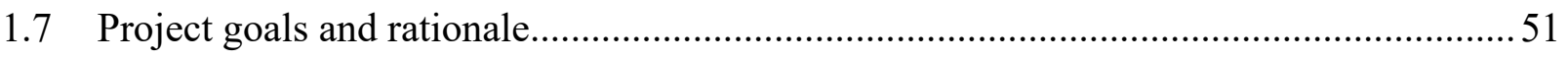

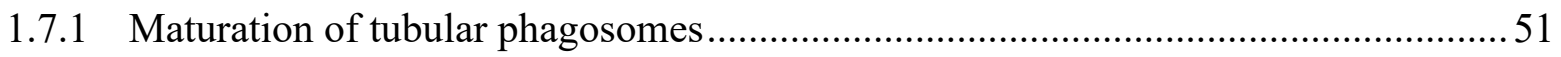

1.7.2 Lysosome adaptation and remodelling in activated phagocytes .............................52

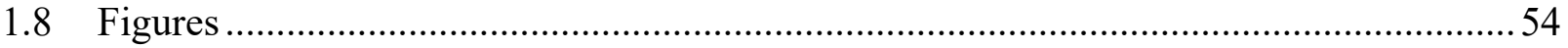

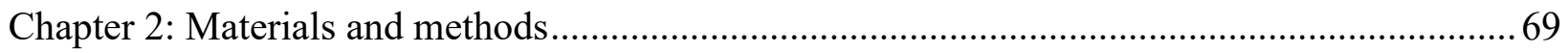

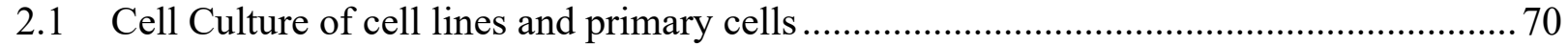

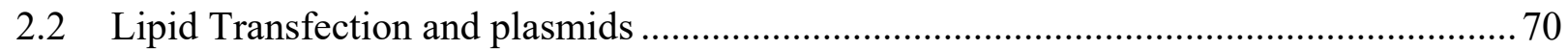

2.3 Preparation of pHrodo-conjugated filamentous bacteria........................................... 71

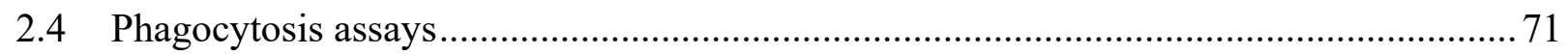

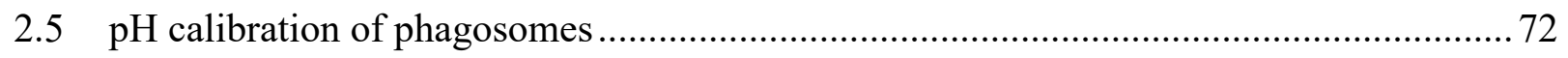

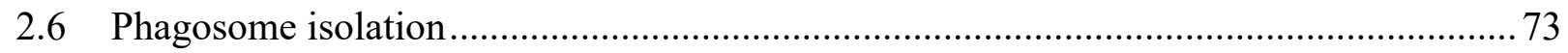

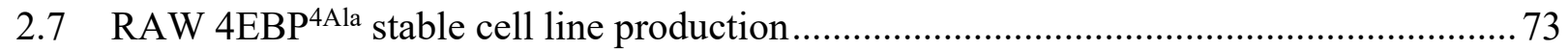

2.8 Rate, retention and accumulation of pinocytic probes ............................................. 74

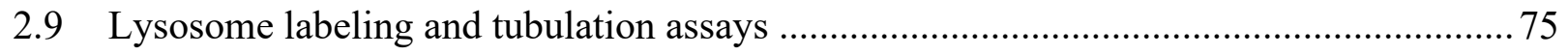

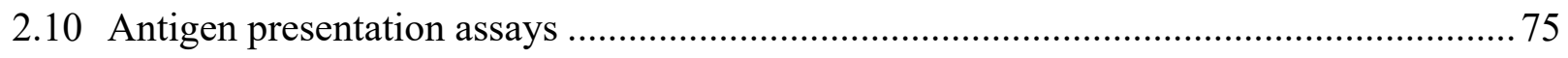

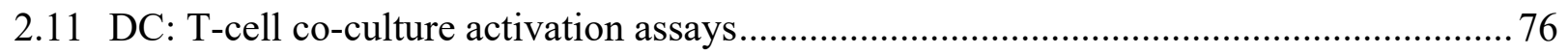

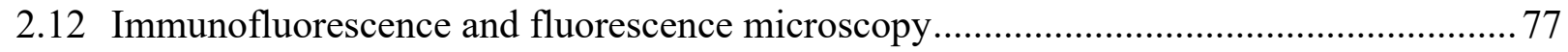

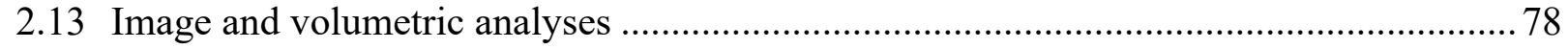

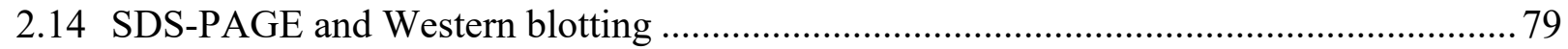

2.14.1 Determination of phagosome-associated proteins and Phos-tag ${ }^{\mathrm{TM}}$ gel assays .......... 79

2.14.2 Puromycylation assay and lysosome biogenesis markers ...................................... 80

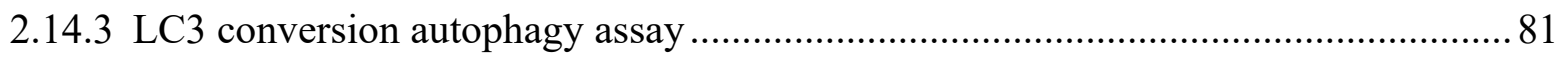

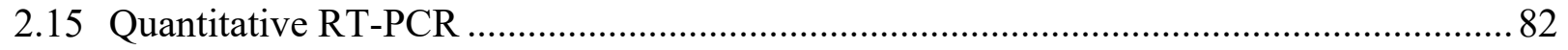

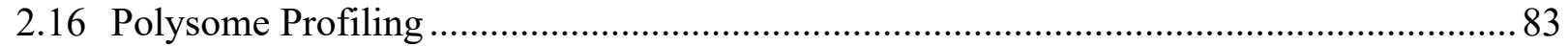

2.17 Phosphoinositide labeling and HPLC-coupled flow scintillation ................................ 84

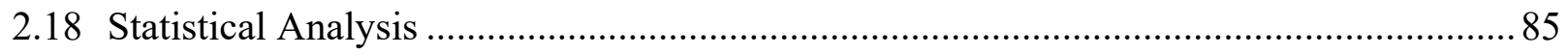

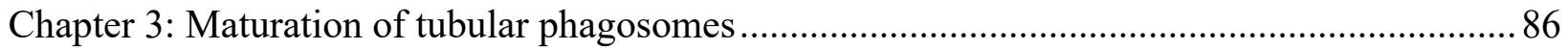

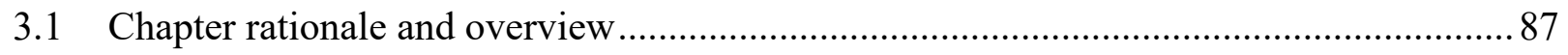

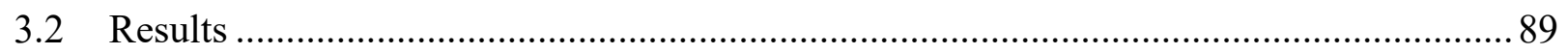


3.2.1 Vps34 controls PtdIns(3)P persistence on tPCs during maturation .........................89

3.2.2 PtdIns(3)P loss at distal tips of tPCs correlates with local acidification .................. 91

3.2.2.1 Acidification of tPCs correlates with the loss of PtdIns(3)P ........................ 92

3.2.2.2 Luminal $\mathrm{pH}$ of 6.2 controls PtdIns(3)P turnover on tPCs.............................. 93

3.2.3 pH controls the lifespan of PtdIns(3)P on canonical phagosomes ......................... 95

3.2.3.1 Luminal $\mathrm{pH}$ between 6.1-6.3 controls PtdIns(3)P turnover on phagosomes .... 96

3.2.4 Vps34 association onto membranes controls PtdIns(3)P cellular levels ...................98

3.2.4.1 Luminal $\mathrm{pH}$ controls Vps34 association onto membranes ............................98

3.2.4.2 Vps34 controls PtdIns(3)P cellular levels on endosomes ............................. 99

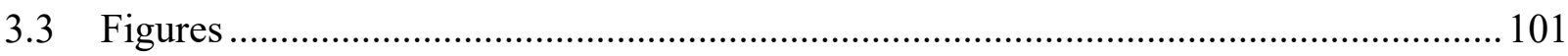

Chapter 4: Lysosome adaptation and remodelling in activated phagocytes ............................ 118

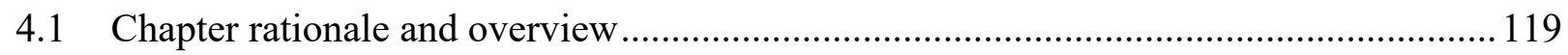

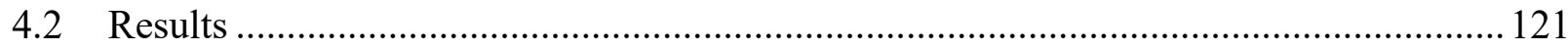

4.2.1 Acute LPS-activation of phagocytes remodels the lysosome population ............... 121

4.2.1.1 Activation of macrophage and dendritic cells expands lysosome volume ..... 121

4.2.1.2 Phagocyte activation increases lysosomal holding capacity ......................... 122

4.2.2 mTOR modulates LPS-induced lysosome remodelling ...................................... 125

4.2.2.1 Activated macrophages express higher levels of lysosomal proteins ............ 125

4.2.2.2 Rapid lysosome expansion is not dependent on TFEB and TFE3 ................. 126

4.2.2.3 Rapid lysosome expansion depends on AKT and mTOR activity ................ 128

4.2.3 mTOR bolsters immunity by enhancing translation of lysosomal transcripts ......... 130

4.2.3.1 LPS boosts global protein synthesis in primary macrophages through mTOR 130

4.2.3.2 mTOR increases translation of mRNAs encoding lysosomal proteins........... 130

4.2.3.3 4E-BP1 and S6 kinase regulate lysosome expansion in activated phagocytes 131

4.2.3.4 LPS promotes Ag-presentation and T-cell activation through mTOR and S6K

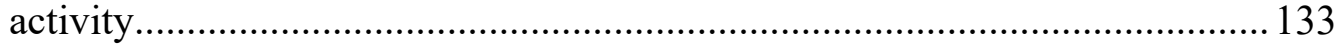

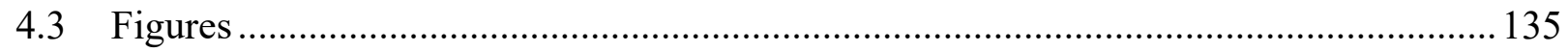

Chapter 5: Lysosome adaptation in adjuvant-activated antigen presenting cells...................... 157

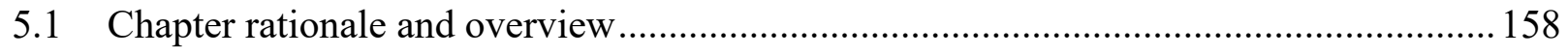

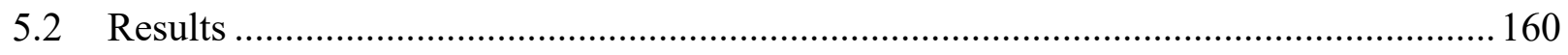

5.2.1 Select adjuvants can remodel the lysosome population ...................................... 160

5.2.2 Adjuvants bolster antigen presentation through mTOR and S6K........................ 162 
5.2.3 Adjuvants bolster T-cell activation through mTOR and S6K 164

5.3 Figures. 166

Chapter 6: Discussion. 175

6.1 The tubular phagocytic cup as a model to study phagosome maturation

176

6.1.1 PtdIns(3)P remains on tPCs regardless of other early and late endosomal markers 176

6.1.2 Phagosome acidification controls Vps34 complex II association and PtdIns(3)P levels 178

6.1.3 How can luminal $\mathrm{pH}$ control Vps34 association onto phagosomal membranes? .... 180

6.1.4 The tubular phagocytic cup as a model to study phagosome maturation 183

6.2 Lysosome remodelling in activated phagocytes. 186

6.2.1 Acute phagocyte activation bolsters lysosomal volume and holding capacity 187

6.2.2 mTOR-S6K-4E-BP axes control lysosome expansion 190

6.2.3 Lysosome remodelling during vaccine response. 194

6.2.4 Biochemical properties and function of lysosomal tubulation. 196

6.3 Figures 202

Chapter 7: Conclusion 213

Appendix 216

References 232 


\section{List of figures}

Figure 1.1: The lysosome is a convergence point for multiple membrane trafficking pathways in

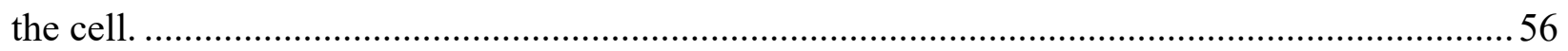

Figure 1.2: Phosphoinositides and GTPases confer organelle identity .......................................58

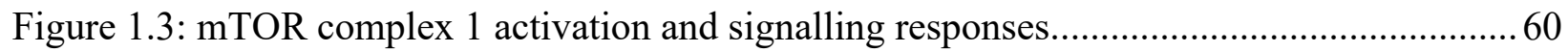

Figure 1.4: Lipopolysaccharide signals through the toll-like receptor 4 (TLR4) signalling pathway.

Figure 1.5: Phagosome maturation of canonical and tubular targets. 64

Figure 1.6: Schematic of antigen-major histocompatibility complex II presentation in dendritic cells to $\mathrm{CD} 4^{+} \mathrm{T}$-cells. 66

Figure 1.7: Proposed model of the known signalling pathway and mediators of lysosome tubulation. .68

Figure 3.1: Non-canonical persistence of PtdIns(3)P on tPCs during maturation. 103

Figure 3.2: Vps34 activity is responsible for PtdIns(3)P persistence on tubular phagocytic cups.

Figure 3.3: PtdIns(3)P dynamics during tPC internalization. 106

Figure 3.4: Luminal $\mathrm{pH}$ and V-ATPase activity control PtdIns(3)P dynamics on tPCs. 108

Figure 3.5: tPC luminal $\mathrm{pH}$ of 6.2 causes the dissociation of PtdIns(3)P probes. 109

Figure 3.6: $\mathrm{pH}$ neutralization causes PtdIns(3)P to persists in phagosomes. 110

Figure 3.7: $\mathrm{p} 40 \mathrm{PX}-\mathrm{mCh}$ recruitment to zymosan-containing phagosomes is $\mathrm{pH}-$ dependent. .... 112 Figure 3.8: Phagosomal luminal pH between 6.1-6.3 causes dissociation of PtdIns(3)P probes.

Figure 3.9: The Vps34 complex II association to phagosomes is controlled by $\mathrm{pH}$. 115

Figure 3.10: PtdIns(3)P on endosomes is controlled by $\mathrm{pH}$. 117

Figure 4.1: LPS-activation augments lysosome volume in phagocytes. 137

Figure 4.2: Super-resolution microscopy showing expanded lysosome network. 138

Figure 4.3: Macrophage activation increases lysosomal holding capacity.

Figure 4.4: Activated RAW macrophages have a larger lysosome holding capacity. 141

Figure 4.5: Lysosome remodelling requires protein biosynthesis. 143

Figure 4.6: Lysosome remodelling is independent of TFEB and TFE3 activation. 145

Figure 4.7: mTOR stimulates lysosome volume and holding capacity. 147 
Figure 4.8: LPS stimulates global protein synthesis in primary macrophages.

Figure 4.9: LPS increases translation of mRNAs encoding lysosomal proteins in an mTORdependent manner.

Figure 4.10: S6 kinase is required for the LPS-mediated lysosome expansion.

Figure 4.11: Active 4E-BP1 suppresses LPS-mediated lysosome expansion.

Figure 4.12: mTOR and S6 kinase control E $\alpha_{52-68}$ peptide presentation in activated BMDCs. . 156

Figure 5.1: Select adjuvants induce lysosome remodelling through mTOR. 167

Figure 5.2: Compound A and B enhance HEL-MHCII presentation through mTOR.

Figure 5.3: Compound A and B enhance E $\alpha$-MHCII presentation through mTOR and S6K... 171

Figure 5.4: Adjuvant complexing can enhance Ag-presentation efficacy. 173

Figure 5.5: Lysosome remodelling in adjuvant-activated DCs enhances T-cell activation. 174

Figure 6.1: Schematic illustrating stages of tubular phagocytic cup maturation and PtdIns(3)P dynamics. 204

Figure 6.2: Schematic illustrating signalling pathways and molecular mediators that control lysosome remodelling in activated phagocytes. 206

Figure 6.3: Fluid-phase cargo traffic to tubular and punctate lysosomes at the same rate. .208

Figure 6.4: Luminal properties in tubular and punctate lysosomes differ in activated phagocytes. 210

Figure 6.5: Tubular lysosomes may be more proteolytic than punctate lysosomes in macrophages. 


\section{List of appendices}

Figure A1: PI3K and tPC length-dependent recruitment of 2FYVE-GFP and p40PX-GFP.....217

Figure A2: Loss of PtdIns(3)P is correlated with the acidification of distal tPCs..................218

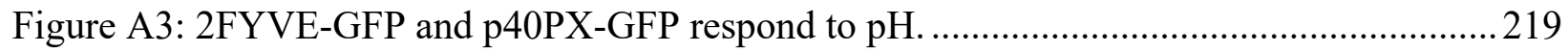

Figure A4: LPS increases lysosomal protein synthesis through mTOR and S6K..................221

Figure A5: Basal lysosome properties and trafficking is indistinguishable in wild-type RAWs and

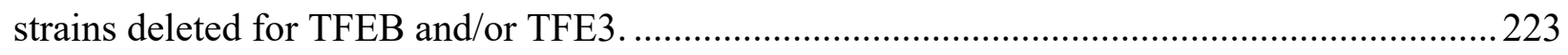

Figure A6: Impact of pharmacological inhibitors on autophagy induction and LY258470 treatment on S6K activity during and general protein synthesis.....................................................225

Figure A7: The effects of Torin1 on RAW macrophage protein synthesis. ...........................226

Figure A8: Polysome profiling of RAW macrophages: additional replicate data. ...................228

Figure A9: Effect of LY258470 and Torin1 treatments on HEL presentation by BMDCs........229

Figure A10: Adjuvant-induced lysosome remodelling supporting data. ................................231 


\section{List of abbreviations}

4E-BP1 - Eukaryotic initiation factor 4E (eIF4E)- binding protein 1

5'TOP -5 ' terminal oligopyrimidine

Ag - Antigen

AKT/PKB - RAC-alpha serine/threonine-protein kinase/ Protein kinase B

ALR - Autophagic lysosome reformation

AMP - Adenosine monophosphate

AMPK - AMP-activated protein kinase

APC - Antigen presenting cell

Arl - Arf-like

ATG13/9 -Autophagy related protein 13/9

ATP - Adenosine triphosphate

ATP6V1D/H - ATPase $\mathrm{H}^{+}$Transporting V1 Subunit D/H

$\mathrm{BMDC}$ - Bone marrow-derived dendritic cell

BMDM - Bone marrow-derived macrophage

cDNA - Complementary DNA

CLEAR - Coordinated lysosomal enhancement and regulation

CLIP - Class II-associated invariant chain peptide

CMA - Chaperone mediated autophagy

ConA - Concanamycin A

CTL - Cytotoxic/ $\mathrm{CD}^{+} \mathrm{T}$-cell

DAMPS - Damage-associated molecular patterns

DC - Dendritic cell

ECM - Extracellular matrix

EEA1 - Early endosome antigen 1

eIF3 - Eukaryotic initiation factor complex 3

eIF4E/G/2/4G/4A/4F/4B - Eukaryotic initiation factor 4E/G/2/4G/4A/4F/4B

ER - Endoplasmic reticulum

F-Lp - Filamentous legionella pneumophila

FYCO1 - FYVE and coiled-coil domain-containing protein 1

FYVE - (Fab1, YOTB, Vac1, EEA1) 
GAP - GTPase-activating protein

GEF - Guanyl exchange factor

GFP - Green fluorescent protein

GTPase - Guanosine triphosphatase

HEL - Hen egg lysozyme

IgG - Immunoglobulin G

I $\mathrm{B}$ - Inhibitor of kappa B

IKK - IкB kinase

IL - Interleukin

KIF5 - Kinesin family member

LAMP - Lysosome associated membrane protein

LC3 - Light Chain 3

LIMP - Lysosome integral membrane protein

Lp - Legionella pneumophila

LPS - Lipopolysaccharide

LY - Lucifer yellow

LY2 - LY258470

MAMPS - Microbe-associated molecular patterns

$\mathrm{mCh}-\mathrm{mCherry}$ fluorescent protein

MHCII - Major histocompatibility complex II

MIIC - MHC class II compartment

mRNA - Messenger ribonucleic acid

mTOR - Mechanistic target of rapamycin

mTORC $1 / 2$ - mTOR complex $1 / 2$

MVB - Multivesicular body

MyD88 - Myeloid differentiation primary response protein 88

NFкB - Nuclear factor kappa-light-chain-enhancer of activated B cells

NK - Natural killer cells

NLRP3 - Nod-like receptor family pyrin domain containing 3

NLS - Nuclear localization sequence

NOD - Nucleotide-binding oligomerization domain 
pHRodo-Lp - pHRodo conjugated- legionella pneumophila

PI3K - Phosphatidylinositol 3-kinase

PIKfyve - Phosphoinositide kinase, FYVE-type zinc finger containing

PM - Plasma membrane

PMA - Phorbol myristate acetate

PRR - Pattern recognition receptors

PtdIns - Phosphatidylinositol

PtdInsP - Phosphoinositide

PX - Phox homology

qRT-PCR/qPCR - quantitative real--time polymerase chain reaction

$\mathrm{Rab}$ - Ras- related protein

Raptor - Regulatory associated protein of mTOR

Rheb - Ras homolog enriched in the brain

Rictor - Rapamycin insensitive component of TOR

RILP - Rab7-interacting lysosomal protein

RNA - Ribonucleic acid

RNA-seq-RNA-sequencing

ROS - Reactive oxygen species

S6K - S6 kinase

SD - Standard deviation

SEM - Standard error of the mean

Ser/Thr - Serine/threonine

SLC15A3 - Solute carrier family 15 member 3

SLC38A9 - Solute carrier family 38 member 9

SREBP1 - Sterol regulatory element-binding transcription factor 1

TCR - T-cell receptor

TFEB/3 - Transcription factor EB or 3

TL - Tubular lysosome

TLR - Toll-like receptor

TNF $\alpha$ - Tumor necrosis factor $\alpha$

tPCs - Tubular phagocytic cup 
TRPML1 - Transient receptor potential mucolipin-1

TSC $1 / 2$ - Tuberous sclerosis protein $1 / 2$

ULK1 - UNC-51-like kinase 1

UPR - Unfolded protein response

UTR - Untranslated region

UVRAG - UV radiation resistance-associated gene protein

V-ATPase - Vacuolar- $\mathrm{H}^{+}$ATPase

Vps15/34 - Vacuolar protein sorting 15 or 34 
Chapter 1: Introduction 


\section{$1.1 \quad$ Dissertation overview}

In this thesis document I present and discuss the findings of two major research projects that identify novel mechanisms of organelle identity and adaptation in phagocytic immune cells.

In Chapter 1, I begin the introduction by presenting key membrane proteins and lipids that help to establish organelle identity in eukaryotic cells. I focus on their role in endomembrane pathways and feature the mammalian target of rapamycin complex 1 (mTORC1) as an important signalling complex that coordinates cellular homeostasis in response to cellular stress, energy levels and more. I follow-up with the concept that cells are highly adaptive in response to environmental cues modulating cellular state and function. I introduce phagocytic immune cells as an example cell-type that is highly adaptive and describe their role in innate immunity. I explain how phagocytes recognize extracellular cargo, how they respond intracellularly by adapting the endomembrane system; including upregulating phagocytosis and antigen presentation. I describe how in nature, immune cells are challenged with particles of disparate shapes and how this impacts phagocytosis. I then highlight the importance of the lysosome, a central organelle in phagocyte immune function, describing its biochemical properties and function in immunity. Finally, I discuss the phenomenon of lysosome tubulation, proposed functions in innate immune cells and current knowledge of their biogenesis and regulation. Chapter 1 concludes with the two main rationales and objectives for this thesis.

In Chapter 2, I provide descriptions of the experimental procedures involved in carrying out this research. The techniques used include but are not limited to culturing cell-lines, cells derived from murine bone-marrow and generating stable cell lines. I describe various live- and fixed-cell fluorescence microscopy techniques and equipment used to carry out this work. In addition, I describe the molecular biology techniques, like lipid-transfection, western blotting and 
quantitative polymerase chain reaction (qPCR) used to overexpress transgenic proteins and detect protein and gene expression levels, respectively. Finally, various analysis protocols are outlined in combination with image and statistical analysis software.

My first main project is described in Chapter 3, beginning with a brief overview of previous work elucidating the mechanisms of tubular phagocytic cup (tPC) maturation and the rationale for my work. I present data uncovering a novel $\mathrm{pH}$-dependent mechanism that controls phosphatidylinositol-3-phosphate (PtdIns(3)P) levels on the maturing tPC, which contravenes the canonical pathway of spheroidal targets. We identify that phagosome luminal $\mathrm{pH}$ determines Vps34 complex II association onto the membrane and PtdIns(3)P dynamics. This project was completed in collaboration with Amriya Naufer from University of Toronto, Scarborough, and resulted in a first co-authorship article published in the Journal of Cell Biology (Naufer, Hipolito, et al., 2018).

My second major project is described in Chapter 4, which I begin by providing a brief background on lysosome tubulation, their function in immune cells and discuss the rationale for my research. The data I present elucidate a novel mechanism controlling lysosomal adaptation and remodelling in immune cells. First, we show that acute lipopolysaccharide (LPS) activation in phagocytes, bolsters lysosomal holding capacity and volume. Second, we identify a novel mTORdependent mechanism whereby lysosome remodelling is driven through enhanced lysosomal mRNA translation. We show that the mTOR-S6K axis is required for enhanced antigen presentation in activated phagocytes. A manuscript of this work was submitted to PLOS Biology, and we are currently completing the revisions requested by reviewers.

In Chapter 5, I investigate lysosome remodelling in antigen presenting cells in the context of vaccine response. I begin with a brief overview and discussion of the role of adjuvants in 
vaccines, and their link towards natural antigens. I provide preliminary work identifying a novel role for lysosome remodelling in modulating the immunostimulatory effects of adjuvants currently used in vaccines. I show that select adjuvants are able to induce lysosome remodelling and antigen presentation, which may depend on the mTOR-S6K pathway.

Finally, I culminate the thesis with a discussion of the major projects described in Chapters 3 and 4. In addition to describing the clinical implications of lysosome tubulation and remodelling based on the preliminary work presented in Chapter 5, I propose and outline future experiments that may begin to clarify the protein players that regulate these pathways and recommend investigating the biochemical properties of lysosomal tubulation to better elucidate their function. Ultimately, I provide concluding remarks on the impact of my research in the immune cell biology field and its potential impact in vaccine design. 


\section{Organelle identity and transport}

The cytoplasm of eukaryotic cells is complex, containing an array of functional microenvironments. The cytoplasm achieves functional order through compartmentalization of protein complexes and signalling networks into defined organelles. Organelles can be membranebound, enclosed in a membrane bilayer such as the mitochondria and the endoplasmic reticulum (ER), or membraneless ordered complexes, achieved through liquid-liquid phase separation, such as nucleoli and P-bodies (Gomes \& Shorter, 2018; Shorter, 2016). Compartmentalization not only achieves cellular order, but it also helps to separate competing cellular reactions such as protein synthesis and degradation in the ER and lysosomes or proteasomes, respectively. Organelles are not functionally exclusive and static, they are constantly exchanging content and communicating to maintain cellular homeostasis. Inter-organelle communication is achieved through regulated mechanisms like vesicular trafficking, organelle-organelle contact sites and protein signalling networks. Next, I will introduce key topics at a high-level and follow with detailed sections on key topics. Described here is endocytosis, an example of vesicular trafficking, where plasma membrane receptors and content from the extracellular milieu (together called cargo) are transported into the cell (Figure 1.1).

\subsubsection{Membrane trafficking through the endo-lysosomal system}

The process of endocytosis encompasses a series of membrane fission and fusion events. Plasma membrane receptors and extracellular material are internalized through invaginations of the plasma membrane. This may or may not occur with the membrane coat protein clathrin, and an abundance of adaptor proteins. Following inward membrane invagination, a vesicle will pinch off forming an endosome containing cargo destined for a variety of subcellular functions. The endosome is 
trafficked and fuses with the early endosome. The early endosome, is a vesicular sorting intermediate, which forms tubular microdomains of the internalized cargo destined for recycling back to the plasma membrane or intracellular degradation (Abrahamson \& Rodewald, 1981). The tubular extensions fission, forming new vesicular intermediates that can be trafficked back to the plasma membrane, as is the case with transferrin receptor (Harding, Heuser, \& Stahl, 1983), or marked for degradation.

Early endosomal cargos destined for degradation are post-translationally modified with ubiquitin proteins onto the cytosolic portion of the cargo molecules, through covalent addition onto lysine residues. Proteins can be mono- or poly-ubiquitinated, yielding differential fates; typically, proteins destined for degradation are poly-ubiquitinated. This ubiquitin tag serves as a marker to recruit the Endosomal Sorting Complexes Required for Transport (ESCRT) machinery. Upon recruitment, the ESCRT machinery deforms the endosomal membrane by forming a supramolecular complex which induces membrane invagination of cargo, destined for degradation, into intraluminal vesicles (ILVs) within the endosome (Katzmann, Babst, \& Emr, 2001; Migliano $\&$ Teis, 2018). During this process, early endosomal markers are shed and late endosomal markers are acquired demarcating endosomal maturation (Rink, Ghigo, Kalaidzidis, \& Zerial, 2005), expanded in Section 1.4.1.1. After, the late endosomal compartment finally fuses with the lysosome where the cargo is degraded.

The lysosome functions as the degradative hub in several endocytic and membrane trafficking pathways (Figure 1.1). The lysosomal lumen is specialized as it maintains an acidic $\mathrm{pH}$ between 4.5-5.5, through the action of the vacuolar $\mathrm{H}^{+}$pump (V-ATPase), which establishes an environment optimal for many hydrolytic enzymes that drive the degradation of a variety of macromolecules including proteins, carbohydrates, lipids and nucleic acids. The resulting 
degradation products, like amino acids, can then be reused by the cell and transported out of the lysosome through numerous transporters (Schröder, Wrocklage, Hasilik, \& Saftig, 2010). These by-products are used as substrates in several cellular pathways that maintain cellular homeostasis. As a major theme of my thesis, I provide an in-depth overview of lysosomal physiology and function in section 1.5.

During endocytosis and other membrane trafficking pathways, several vesicular intermediates and organelles undergo a multitude of membrane intermixing and coalescence. Nevertheless, the vesicular intermediates and organelles are able to maintain their distinctiveness by recruiting molecules that facilitate trafficking, in addition to, serving as organelle identity markers. Most notably are the phosphoinositide lipids and small GTPases (Figure 1.2).

\subsubsection{Phosphoinositides}

Phosphoinositides (PtdInsPs) are low abundance phospholipids involved in a variety of cellular functions such as cell motility, signal transduction and membrane trafficking. Phosphoinositides are derived from the parental species phosphatidylinositol (PI), whose inositol headgroup can be phosphorylated to give rise to a variety of species. These lipids are found on the inner and outer leaflets of the plasma membrane and a variety of organelles, constituting $10-20 \%$ of all phospholipids (Balla, 2013). Structurally, the glycerol backbone contains two fatty acid tails and a myo-inositol headgroup, with the most common fatty acid tail combination as 1-stearoyl-2aracadonyl (Figure 1.2a). Moreover, the number of carbons and/or degree of saturation or unsaturation can differ within and across species, conferring molecular heterogeneity and can impact preference of effector binding and downstream signalling (Bone et al., 2017; Schmid, et al., 2004; Shulga, et al., 2012) . 
In mammalian cells, eight and ten classes of kinases and phosphatases, respectively, give rise to seven PtdInsP species by reversible phosphorylation at the $3^{\text {rd }}, 4^{\text {th }}$, or $5^{\text {th }}$ position yielding three mono- $[\mathrm{PI}(3) \mathrm{P}, \mathrm{PI}(4) \mathrm{P}, \mathrm{PI}(5) \mathrm{P}]$, three bis- $\left[\mathrm{PI}(3,4) \mathrm{P}_{2}, \mathrm{PI}(3,5) \mathrm{P}_{2}, \mathrm{PI}(4,5) \mathrm{P}_{2}\right]$ and one trisphosphorylated species $\left[\mathrm{PI}(3,4,5) \mathrm{P}_{3}\right]$ (Balla, 2013; Takehiko Sasaki et al., 2009). Phosphoinositides can function as secondary messengers in signal transduction pathways and/or recruit and dock effector proteins to membranes, facilitating the formation of signalling complexes. Each phosphoinositide is found localized to a particular subcellular organelle to facilitate basal function, where some can also be made locally to support cellular signalling events on alternative organelles. For example, $\mathrm{PI}(4,5) \mathrm{P}_{2}$ is found localized at the plasma membrane to help facilitate clathrin mediated endocytosis (Abe, et al, 2008), but can also be transiently produced during the budding of proto-lysosomes during the process of autolysosome reformation (Y. Rong et al., 2012). Both the differential localization and quick interconversion renders these phospholipids ideal markers for membrane identity and mediators of signalling.

Phosphoinositides act as membrane markers, whereby they recruit protein effectors which often contain specific PtdInsP binding domains that bind with high affinity to the phosphorylated headgroup. For example, $\mathrm{PI}(4,5) \mathrm{P}_{2}$ localized at the plasma membrane recruits epsin via the ENTH (epsin $\mathrm{NH}_{2}$ - terminal homology) domain (Itoh et al., 2001; Legendre-Guillemin, Wasiak, Hussain, Angers, \& McPherson, 2004). Upon recruitment, epsin localization facilitates membrane deformation and the recruitment of adaptor proteins necessary for clathrin-mediated endocytosis. During endocytosis, early endosomes are decorated with PI(3)P, which recruits other endosomal markers Rab5 and EEA1 (early endosome antigen 1), via their FYVE (Fab1, YOTB, Vac1, EEA1) domain, as well as, the lipid kinase, PIKfyve, facilitating its turnover to $\mathrm{PI}(3,5) \mathrm{P}_{2}$ and ultimate maturation to a late endosomal compartment (Burd \& Emr, 1998; Gary, Wurmser, Bonangelino, 
Weisman, \& Emr, 1998) (See "Phagocytosis and phagosome maturation"). Absence of these signalling lipids impairs the progression of the pathway they activate.

The subcellular distribution of phosphoinositide species is not as simple as what is currently acknowledged. In fact, many PIs are localized promiscuously in the cell leading to potential issues in effector specificity and recruitment (Figure 1.2c). Currently the accepted model explaining effector specificity is based on coincidence detection, where effector localization and behaviour depends on PtdInsP recruitment and requires an additional secondary signal on the target membrane (Carlton \& Cullen, 2005). For instance, EEA1 requires binding to PtdIns(3)P and GTPbound Rab5 in order to associate with and tether PtdIns(3)P- and Rab5-positive endosomes to induce homotypic endosome fusion (Lawe et al., 2002). However, acyl chain composition may provide an additional layer for effector specificity. Whereby, differences in acyl chain composition may affect effector interaction and binding to the polar inositol headgroup present on target membranes. Interestingly and in support of this, one can appreciate that some of the biosensors used to detect PtdInsPs recognize certain pools of the target, such as the PH domain derived from FAPP1, which only detects PtdIns(4)P in the Golgi and not including the plasma membrane (Hammond, Machner, \& Balla, 2014). Differences in acyl chain composition of the same PtdInsP species present on different organelles may, therefore, explain the differences in detection and effector specificity.

The coordination of PtdInsP headgroup phosphorylation, acyl chain content and specificity of effector recruitment to the target membrane confers organelle identity and the controlled activity of the signalling pathways they regulate. 


\subsubsection{Small GTPases and their effectors}

Members of the Ras superfamily of monomeric guanosine triphosphatases (monomeric or small GTPases) also serve as markers for membrane identity, due to their switch-like nature. In general, most small GTPases contain a core effector binding domain and G-domain (Wittinghofer, 2014). Sequence variation within the $G$ domain is used to separate proteins into subfamilies, but the majority contain switch I and switch II motifs, which are critical for GTP/GDP binding and GTPase activity.

Small GTPases exist in an active or inactive state when bound to GTP or GDP, respectively (Figure 1.2b). In order for GTPases to turn on, guanine nucleotide exchange factors (GEFs) facilitate the displacement of GDP from the GTPase and subsequent loading of ubiquitous GTP. When GTP-bound, GTPases are able to act on downstream effector proteins through non-covalent interactions. GEFs impart their function by binding to the GDP-GTPase interface, destabilizing the interaction and permitting GTP loading onto the empty pocket. Upon GTP binding, the $\gamma$ phosphate of GTP interacts directly with the conserved switch I and switch II motifs, stimulating a conformation change which is favorable for effector binding. GTPases are inactivated when their intrinsic GTPase activity is activated by GTPase activating proteins (GAPs), which help induce the hydrolysis of GTP by stabilizing the transition state and by possibly contributing residues for the hydrolysis in the process (Scheffzek et al., 1997).

The Rab family of GTPases, a member of the Ras superfamily, plays a critical role in trafficking, organelle identity and function within the endolysosomal system (Figure 1.2c). Rab GTPases differentially localize to endocytic compartments; where Rab5, Rab11 and Rab7 are localized to early endosomes, surfaces of recycling endosomes and to late endosomes, respectively (Grosshans, Ortiz, \& Novick, 2006). These molecular markers act as molecular switches that 
control endosomal and phagosomal maturation (See "Phagocytosis and phagosome maturation" for role of Rab GTPases and their cycling during endolysosomal remodelling). Similarly, the Arf family of proteins also regulate dynamics of the endolysosomal system, such as recruiting the molecular motor machinery required for vesicular transport (Nie, Hirsch, \& Randazzo, 2003). Rab and Arf GTPase recruitment and activity on target membranes is tightly linked to their nucleotide bound state.

When GTP-bound Rab and Arf GTPases are linked to the target membrane through a prenyl and myristate modification, respectively. Following GTP hydrolysis, Arf GTPases undergo a conformational change which displaces the myristate group, leading to its dissociation from the membrane. When Rab GTPases are GDP-bound they are susceptible to membrane removal by GDP dissociation inhibitor (GDI) (Sasaki et al., 1990). The GDI protein binds to Rab-GDP, masking the prenyl modification and solubilizing the inactive form into the cytosol. Both inactive forms of Rab and Arf GTPases provide a pool of inactive switches for the next round of activation. The localization and switch-like nature of GTPases are important for coordinating effector recruitment to target organelles, initiating signalling complex formation, coordinating the recruitment of molecular motor machinery and more (Figure 1.2c). The ability of GTPases to act as 'molecular switches' is critical for membrane trafficking and for conferring identity to the compartments they bind. In order to fine-tune cellular function, the cell must harmonize both internal and external cues, by modulating the molecular identity of organelles and coordinating the activity of protein complexes. 


\subsubsection{Subcellular response to intrinsic and extrinsic stimuli}

In addition to synchronizing molecular switches and protein signalling pathways in response to signalling cues, cells can adapt their fate depending on context. Extrinsic signals, such as growth factors, signal through growth factor receptors found on the plasma membrane (e.g. epidermal growth factor receptor) promoting cellular growth and proliferation. Likewise, the cell can respond to its intracellular state by activation of cytoplasmic signalling networks, such as those that promote cell migration during instances of cell growth. In contrast, cells may receive a signal promoting programmed cell death when infected or under a compromised energy state. Together extrinsic and intrinsic signals can promote a variety of signalling cascades that dictates a cell's response.

These signals can also dictate organelle adaptation and function. For example, mitochondria in yeast cells grown in glycerol medium increase the volume and undergo tubulation, forming a homogenized network to compensate for their increased demand in oxidative phosphorylation. Inhibition of this remodelling greatly impacts mitochondrial DNA integrity, cellular responses to stress and respiratory capacity (Chen et al., 2010; Egner, Jakobs, \& Hell, 2002). Another example of organelle adaptation is the reorganization of the endoplasmic reticulum during B-cell maturation. As B-cells undergo differentiation into plasma cells, which are largely secretory, the ER increases in biogenesis and volume to compensate for the increased demand in protein synthesis and secretion (Kirk, Cliff, Thomas, \& Ward, 2010).

Cells are constantly making decisions that coordinate cellular fate and function in response to intrinsic and extrinsic cues. Within the cell there are a variety of sensors that coordinate responses to cellular states by adapting the function of signalling pathways and organelles based on signal context. Described next, in detail, is the mammalian target of rapamycin (mTOR), a 
cellular master switch that integrates the nutrient and energy state of the cell with environmental cues, to control cellular signalling pathways and function. 


\subsection{Mammalian Target of Rapamycin nutrient sensing hub}

The mammalian target of rapamycin (mTOR) is a serine/ threonine kinase that integrates the nutrient status of a cell, and signals for cell growth and proliferation (reviewed in: Guertin \& Sabatini, 2007; Valvezan \& Manning, 2019). mTOR is the catalytic subunit of two major regulatory complexes known as mTOR complex 1 (mTORC1) and 2 (mTORC2). This $280 \mathrm{kDa}$ protein is considered a PI3K-related kinase, as its $\mathrm{C}$ terminal kinase domain resembles the catalytic domain of PI3K. mTORC1 and 2 complex formation are highly regulated and controlled by a number of signalling pathways. Due to the focus of this thesis, I focus on mTORC1 complex formation, regulation and function. See Figure 1.3 for a detailed schematic of known signalling pathways that feed into and regulate mTORC1 activity, including its downstream targets. Below I highlight some of the major signals and pathways that control mTORC1 activity.

\subsubsection{Complex formation and signalling}

The mTORC1 five-subunit complex is composed of the core mTOR kinase, associated with Raptor (regulatory associated protein of mTOR), PRAS40 (proline-rich AKT1 substrate of $40 \mathrm{kDa}$ ), mLST8 (mammalian lethal with Sec13 protein 8) and Deptor (DEP-domain-containing mTORinteracting protein). The function of all the regulatory subunit remains to be fully understood, but they each participate as positive or negative regulators. Raptor is known as a TOR-scaffold, supporting substrate recruitment that drives mTORC1-dependent pathways (Zoncu, Efeyan, \& Sabatini, 2011). Recently, it was shown that Raptor can also control mTOR complex formation and recruitment to the lysosomal membrane via a leucine-sensitive mechanism (Son et al., 2019). The role $\mathrm{mLST} 8$ plays in $\mathrm{mTORC} 1$ regulation is unclear, but is predicted to positively regulate mTOR kinase activity (D.-H. Kim et al., 2003). In contrast, PRAS40 and Deptor are negative 
regulators of mTORC1 function, Deptor directly interacts and blocks the core mTOR kinase (Peterson et al., 2009). Whereas, PRAS40 competes with the binding of mTORC1 and its substrate, under conditions of nutrient deprivation, when its levels are elevated (L. Wang, Harris, Roth, \& Lawrence, 2007).

The mTORC1 complex integrates signals from growth factors, energy status, oxygen levels, amino acids and stress pathways, like immune signalling (Figure 1.3a). Together, these signals coordinate mTORC1 complex formation, localization and signalling. When active, mTORC1 is intimately associated with the endolysosomal organelle, the lysosome. Complex recruitment at the lysosome is influenced by amino acid levels by acting on the heterodimeric Rag GTPases, which bind to nucleotides in opposite states (Sancak et al., 2008). When active, GTPRag A/B heterodimerize with GDP-Rag C/D, this binding is coordinated by amino acid availability. Specifically, amino acid sensors Sestrin2 and CASTOR sense cytosolic leucine and arginine, inhibiting GATOR1 (GAP activity towards Rags), a Rag A/B GAP (L. Bar-Peled et al., 2013; Chantranupong et al., 2016; Wolfson et al., 2016). Additionally, lysosomal amino acids are also sensed and coordinate mTORC1 localization to the lysosomal membrane. Lysosomal arginine levels are sensed by SLC38A9 (Rebsamen et al., 2015; S. Wang et al., 2015) and general lysosomal amino acid levels are sensed by the V-ATPase (Zoncu, Bar-Peled, et al., 2011). Together, they activate the Ragulator complex (Bar-Peled, Schweitzer, Zoncu, \& Sabatini, 2012), a GEF for Rag $\mathrm{A} / \mathrm{B}$, which also serves as a docking site for activated Rag GTPases onto the lysosomal membrane. Lastly, activated Rag GTPases brings mTORC1 to the lysosome allowing for further interactions with Raptor and Ragulator (Sancak et al., 2010).

Following mTOR's localization to the lysosomal membrane, mTORC1 can be activated by Rheb (Ras homolog enriched in the brain) GTPase in response to a variety of signals, such as 
depleted energy and oxygen levels, growth factor stimulation and inflammation (reviewed in: Laplante \& Sabatini, 2009). These pathways lead to mTORC1 activation in a Rheb GTPasedependent manner by negatively regulating the Rheb GAP, tuberous sclerosis complex $1 / 2$ (TSC1/2). Inhibition of TSC1/2 leads to GTP-loading of Rheb and activation of mTORC1 (Garami et al., 2003). Once activated, mTORC1 can elicit a number of responses which stimulate cell growth and proliferation. In doing so, mTORC1 promotes many anabolic processes like the biosynthesis of proteins, lipids and organelles, while limiting catabolic processes such as autophagy.

\subsection{2 mTORC1 promotes cell growth and proliferation}

Under conditions promoting cell growth and proliferation, lipid synthesis is enhanced by mTOR (Figure 1.3d). Active mTORC1 promotes the nuclear translocation of the transcriptional regulators SREBP1 (sterol regulatory element binding protein 1) and PPAR $\gamma$ (peroxisome proliferatoractivated receptor- $\gamma$ ), driving the expression of genes encoding proteins involved in lipid and cholesterol homeostasis (J. E. Kim \& Chen, 2004; Porstmann et al., 2008). The mTORC1SREBP1 pathway is predicted to be driven by the phosphatidic acid phosphatase, lipin-1 (Huffman, Mothe-Satney, \& Lawrence, 2002). Lipin-1 is known to be directly involved in

glycerolipid synthesis and was also shown to promote SREBP1 nuclear translocation, following phosphorylation by mTOR (Peterson et al., 2011). How Lipin-1 mediates SREBP1 activity in the nucleus remains an enigma.

During hypoxic conditions and low energy levels (increased AMP:ATP ratio) the cell must switch from signals promoting cell growth and proliferation (Figure 1.3b). Low energy signals promote the activity of AMPK (AMP-activated protein kinase), which senses the intracellular 
energy status. Activated AMPK phosphorylates both TSC1/2 and Raptor, positively and negatively regulating their functions, respectively (Gwinn et al., 2008; Inoki, Zhu, \& Guan, 2003). Together, these signals decrease mTORC1 signalling, cell growth and proliferation. Additionally, mTORC1 coordinates ATP synthesis in the cell by balancing the degree of oxidative phosphorylation and glycolysis through the transcriptional regulator Hif-1 $\alpha$ (hypoxia-inducible factor $1 \alpha$ ) (Land $\&$ Tee, 2007). Hif- $1 \alpha$ is a transcription factor that enhances the expression of genes for anaerobic glycolysis, such as the glucose transporter GLUT1, and glycolytic enzymes (Semenza, Nejfelt, Chi, \& Antonarakis, 1991). Although mTORC1 is inactive during states of hypoxia, mTORC1 fine tunes this response by driving the translation of Hif-1 $\alpha$ transcripts under normoxic conditions. Researchers are now interested in understanding how tumors have co-opted the AMPK-mTOR-HIF1 $\alpha$ pathway to survive under hypoxic conditions and to drive tumor progression (Semenza, 2010).

Furthermore, mTORC1 activity is upregulated under conditions favouring cell growth, such as growth factor stimulation (e.g. insulin signalling) or inflammation (e.g. TNF- $\alpha$ and LPS) (Inoki, Li, Zhu, Wu, \& Guan, 2002; Lee et al., 2007) (Figure 1.3a). During growth factor stimulation, mTORC1 is activated through the canonical insulin and Ras signalling pathways, which converge onto protein kinase $\mathrm{B}$ (PKB or AKT), extracellular-signal-regulated kinase $1 / 2$ (ERK1/2) and p90 ribosomal S6 kinase 1 (RSK1), all of which negatively regulate TSC1/2 activity through phosphorylation. Inflammation is another signal that positively regulates mTORC1 activity, promoting cell differentiation and growth. Pro-inflammatory cytokines such as TNF $\alpha$ can activate IкB kinase- $\beta$ (IKK- $\beta$ ), which physically interacts with and inactivates TSC1/2 (Lee et al., 2007). Under these conditions, mTORC1 enhances protein synthesis to drive pathways that promote immunity and cellular growth. 


\subsubsection{1 mTOR controls the translation of mRNAs}

mTOR signalling promotes protein synthesis at several levels; by direct or indirect regulation of translation initiation machinery, ribosome biogenesis, ribosomal activity and promoting the translation of a subset of mRNA pools (Figure 1.3c). The most well characterized downstream targets of mTOR that mediate mRNA translation are the eukaryotic initiation factor 4E (eIF4E)binding protein 1 (4E-BP1) and the p70 ribosomal S6 kinase 1 (S6K1), which are essential for translation initiation.

The translation initiation complex is under tight regulation by mTOR. The multi-subunit eukaryotic initiation factor complex 3 (eIF3), composed of the small ribosomal subunit eIF2, MettRNA and GTP, acts as a dynamic scaffold for mTORC1 and S6K1 binding (Holz, Ballif, Gygi, \& Blenis, 2005). When mTORC1 is inactive, S6K1 is associated with the eIF3 complex, reducing mRNA translation rates. Additionally, eIF4E, which associates to the 5'-end, is less efficient at recruiting the eIF3 complex for initiation, as 4E-BP1 is bound to it.

Upon activation, mTORC1 is recruited to eIF3 near its two substrates S6K1 and 4E-BP1. Active mTORC1 phosphorylates 4E-BP1 on positions Thr37/46, priming it for subsequent phosphorylation on Ser65 and Thr70, and S6K1 on position Thr389 (Burnett, Barrow, Cohen, Snyder, \& Sabatini, 1998; Fadden, Haystead, \& Lawrence, 1997; Gingras et al., 1999). Following 4E-BP1 phosphorylation, it dissociates from the cap and eIF4G and eIF4A are recruited to eIF4E, assembling the eIF4F complex. Finally, eIF3 (small ribosomal subunit and ternary complex) is recruited, resulting in the complete assembly of the $48 \mathrm{~S}$ translation pre-initiation complex, promoting ribosome scanning and translation initiation for global protein synthesis. Upon complex recruitment, mTORC1 also phosphorylates eIF4G, thus promoting translation, where it acts as a scaffold for the pre-initiation complex and promotes mRNA circularization through interaction 
with 3'-end bound Poly-A binding protein (PABP) (Kahvejian, Svitkin, Sukarieh, M'Boutchou, \& Sonenberg, 2005).

S6K1 priming by mTORC1 is necessary for downstream phosphorylation and activation by phosphoinositide-dependent kinase 1 (PDK1). mTOR-dependent activation of S6K increases ribosome biogenesis and phosphorylation of ribosomal S6 (RS6), which in turn increases ribosomal activity and efficient translation (Jastrzebski, Hannan, Tchoubrieva, Hannan, \& Pearson, 2007). S6K negatively regulates elongation factor 2 kinase (eE2K) through phosphorylation in an mTOR-dependent manner (X. Wang et al., 2001). eE2K phosphorylates and inhibits elongation factor 2 (eEF2), which mediates the translocation step of translation elongation. Together, the several targets of mTOR-dependent phosphorylation promote translational efficiency.

Not only does mTOR control general protein synthesis, there is evidence linking mTOR activity towards selective translation of mRNA pools. One of the pools is known to be "eIF4Fsensitive" mRNAs with long unstructured 5'UTRs. The translation of these mRNAs is sensitive to mTOR inhibition and eIF4F, as they depend on the helicase activity of eIF4A to unwind their complex 5'UTRs (Koromilas \& Sonenberg, 1992; Svitkin et al., 2001). Moreover, it has been shown that S6K phosphorylates eIF4B, which promotes the helicase activity of eIF4A (Holz et al., 2005; Shahbazian et al., 2006). A second pool of mRNAs sensitive to mTOR harbour a terminal oligopyrimidine (TOP) sequence at their 5' end known as a 5'TOP sequence (Avni, Biberman, \& Meyuhas, 1997; Jefferies, Reinhard, Kozma, \& Thomas, 1994). Most 5'TOP mRNAs encode ribosomal and elongation factor proteins, although work has also shown some mitochondrial mRNAs harboring this sequence. It is known that selective regulation of TOP mRNAs by mTORC1 depends on the regulation of eIF4E by 4E-BPs (Miloslavski et al., 2014). It is 
hypothesized that these mRNAs are regulated under stress response and growth conditions and behave as an "all or nothing" phenomenon.

The multifaceted regulation of mTOR and its impact on downstream pathways to promote cellular homeostasis render mTOR a master hub in cellular signalling and homeostasis. Not only does mTOR positively regulate pathways promoting growth and progression, it also negatively regulates catabolic processes like autophagy.

\subsubsection{2 mTORC1 regulation of autophagy}

During nutrient deprived and low energy conditions, autophagy is activated. Autophagy is a cellular degradation and recycling process that is conserved in all eukaryotes and is intimately linked with mTOR. In mammalian cells, there are a three main types of autophagy; microautophagy, macroautophagy and chaperone mediated autophagy (CMA); which all encompass the controlled delivery of cellular cargo into the lysosome for degradation and recycling. During microautophagy, cytosolic cargo is captured through the invagination or protrusion of the lysosomal membrane (Mijaljica, Prescott, \& Devenish, 2011). During CMA, cytosolic chaperone proteins are used to identify cargo proteins containing a consensus motif, directly translocating them into the lysosomal membrane by lysosomal-associated membrane protein 2 (LAMP2) (Bandyopadhyay, Kaushik, Varticovski, \& Cuervo, 2008). Together, these two pathways liberate protein products and energy during instances of cellular starvation and stress.

Macroautophagy, the most well characterized process of autophagy, is regulated by mTOR but differs significantly from microautophagy and CMA. The initiation stage of autophagy occurs within the cytosol, away from the lysosomal membrane and requires de novo synthesis of an intermediate vesicle called an autophagosome. Under nutrient-rich conditions mTORC1 associates 
with the multi-subunit induction complex (ULK1/2-ATG13-RB1CC1) and phosphorylates key autophagy inducers ULK1/2 and ATG13, inactivating them. Under conditions of nutrient starvation whereby mTORC1 is blocked by TSC1/2, mTORC1 dissociates from the induction complex and autophagy proceeds with nucleation (Hosokawa et al., 2009; Kraft et al., 2012). During phagophore nucleation, an array of proteins and lipids are recruited to the phagophore, such as the transmembrane protein ATG9 and the VPS34 complex (Vps34, Vps15, beclin-1 and ATG14L). VPS34 generates localized PtdInsP(3)P on the phagophore, which recruits downstream proteins necessary for initiating phagophore elongation. Next, the cargo is recruited to the inner core of the growing phagophore by autophagy receptors that are associated with the autophagic marker LC3-II (Parzych \& Klionsky, 2014). The phagophore fully expands and encloses the cargo in a double membrane organelle called an autophagosome. Subsequently, autophagosomes matures as they fuse with late endosomes and lysosomes, acquiring a luminal environment that favours macromolecule degradation. The smaller precursors are then retrieved when released from lysosomal transporters and channels, replenishing nutrients during the starvation period.

During autophagy, the lysosomal pool is also upregulated to support the degradative demand by regulation of the transcription factor EB (TFEB), a member of the MiTF/TFE transcription factor family. When amino acids are present, mTORC1 directly phosphorylates TFEB on at least three residues: S122, S142, and S211 (Roczniak-Ferguson et al., 2012). Phospho211 creates a binding site for the chaperone 14-3-3/YHWH, masking TFEB's nuclear localization sequence. As a result, TFEB is restricted to the cytosol and cannot control transcription. Conversely, when amino acids are withdrawn, mTORC1 is inactive and cannot phosphorylate TFEB. Starvation also leads to lysosomal $\mathrm{Ca}^{2+}$ efflux from the lysosomal lumen through the calcium-gated channel TRPML1, into the cytosol (Medina et al., 2015). Release of $\mathrm{Ca}^{2+}$ activates 
the protein phosphatase calcineurin, responsible for the dephosphorylation of TFEB at S211. Dephosphorylation of S211 allows TFEB to enter the nucleus where it can activate the transcription of genes of the coordinated lysosomal enhancement and regulation (CLEAR) network (Roczniak-Ferguson et al., 2012). The CLEAR network is composed of genes encoding lysosomal and autophagy proteins, and is under tight regulation by TFEB and other members of the MiTF/TFE transcription factor family (Palmieri et al., 2011; Sardiello et al., 2009). The boost in transcription and translation of lysosomal and autophagic genes enhances the degradative capacity of the cell to sustain cellular survival during starvation conditions.

Once the cell has returned to a nutrient-rich state, $\mathrm{mTORC} 1$ is reactivated and initiates the process of autophagosome-lysosome reformation (ALR). During ALR, lysosomes used for autophagy begin to bud off from autolysosomes through tubular intermediates, reforming and replenishing the total lysosomal cellular pool, that it had prior to starvation. mTOR catalytic activity is required for this process, as inhibition with rapamycin blocks the formation of tubular lysosomal intermediates, preventing the reformation of lysosomes (Munson et al., 2015; Li Yu et al., 2010).

Markedly, autophagy not only plays an important role in regulating nutrient levels upon states of starvation, but is also intimately linked to pathways controlling the cellular response to stress. One example is mTOR's role in coordinating autophagy during is immunity, whereby, mTOR-autophagy axis is intimately linked to coordinating the immune system response to infection, adapting lymphocyte function and differentiation and more (reviewed in: Deretic \& Levine, 2018). Immune cells like lymphocytes and phagocytes need to constantly adapt and respond to external stimuli, serving as a model for understanding the pathways and protein switches that coordinate cellular survival and adaptation. 


\section{4 $\quad$ Adaptive and innate immune systems}

Organisms have evolved mechanisms of protection from foreign invading particles. Immune defenses can range from simple pathways like the CRISPR/CAS9 system in bacteria to complex systems like mammalian immunity. Virtually all organisms have adapted some mode of protection from foreign material. In higher eukaryotes, including mice and humans, the immune system can be separated into two branches that differ in specificity and outcome but work in concert to defend the host from infection and disease; the innate and adaptive branches.

The innate immune system is thought to be the first line of defense, comprised of physical barriers like epithelial and mucosal layers and non-specific cellular responses mediated by phagocytic leukocytes. Upon infection and breach of the epithelium, an array of chemical signals called cytokines and chemokines are released to recruit leukocytes, including, neutrophils, macrophages and dendritic cells to the site of infection, where they elicit a non-specific immune response. Here, these cells can secrete harmful chemical products like reactive oxygen species to eliminate the invader or internalize the particle and degrade them intracellularly through the process of phagocytosis. The innate branch is intimately linked with the adaptive immune system, whereby it can relay the inflammatory signal to lymphocytes like T-cells and B-cells. The adaptive immune branch contains immunological memory acquired through previous infections, during vaccination or through maternal inheritance. Immunological memory allows for specific defenses upon incoming pathogens through humoral or cell-mediated immunity. Briefly, humoral immunity comes from serum antibodies produced by plasma cells (B-cells) that directly recognize and bind to the target to mark it for clearance. Cell-mediated immunity, also specific, is controlled by cytotoxic T-cells that directly recognize the target and secrete cytotoxic chemicals and mediators 
for clearance. Together, both immune systems work in concert to amplify the signal of infection and the stimulate an inflammatory response (Chaplin, 2010; Iwasaki \& Medzhitov, 2015).

In addition to promoting inflammatory responses, the immune system is made up of regulatory responses that coordinate immune tolerance and promote wound healing after infection. For example, both the adaptative and innate branches work together to fine-tune our immune responses by building tolerance against self-peptides (Bluestone, 2011). Central tolerance is achieved through negative selection of autoreactive $\mathrm{T}$ cells, protecting us from uncontrolled autoimmunity. Moreover, peripheral tolerance is achieved primarily through activation-induced cell death, promoting cell anergy, and inducing regulatory $\mathrm{T}$ cells. In addition, following infection, immune cells (such as phagocytes) are reprogrammed to produce anti-inflammatory responses and promote the circulation and recruitment of specialized cells to the wound. Collectively, they orchestrate and initiate tissue remodelling and healing; reviewed in (Cañedo-Dorantes \& CañedoAyala, 2019). Altogether, the cells of the immune system form a complex system that checks and balances both inflammatory and regulatory responses to maintain homeostasis, when dysregulated or unbalanced diseases such as autoimmunity or chronic infections can arise (Fleisher, 2012; Lehman, 2015; Verbsky \& Chatila, 2014).

To help build immune memory and protection from infection and disease, we have figured out ways to exploit these natural responses using engineered vaccines. In general, vaccines normally contain an inactivated/non-harmful form of the natural antigen, which when introduced stimulate a natural immune response by activating antigen presenting cells and an adaptive response against the pathogen antigen. Vaccines can also contain adjuvants, which are designed to further enhance the immunogenicity of highly purified antigens that have insufficient immunostimulatory capabilities (Di Pasquale, Preiss, Tavares Da Silva, \& Garçon, 2015). 
Adjuvants contribute to the initiation of the innate immune response induced by antigens and can be categorized into carrier or immunomodulatory, the former carrying or creating a depot of antigens and the latter engaging immune receptors directly to recruit and stimulate immune cells (Gutjahr, Tiraby, Perouzel, Verrier, \& Paul, 2016). Although many deadly infectious diseases such as measles and diphtheria have been successfully treated using well-characterized vaccines, there are still many that have not. Therefore, it is still of interest to identify improved antigen-adjuvant pairs that are effective at activating cells of our innate immune system, such as dendritic cells and macrophages.

\subsubsection{Dendritic cells and macrophages in innate immunity}

Dendritic cells (DCs) and macrophages are specialized leukocytes that surveil the host for infection while resident in peripheral tissue and as they distribute through the circulatory system. Upon activation with pro-inflammatory stimuli such as the gram-negative bacterial cell wall component lipopolysaccharide (LPS), or interferon gamma, immune cells undergo reprogramming to boost cellular function and immunity. Activation of classical macrophages and DCs leads to a metabolic switch from oxidative phosphorylation to glycolysis, to increase antimicrobial factors like reactive oxygen species. Increase rates of glycolysis generate energy in the form of ATP and the intermediates necessary to drive processes such as cytokine production, biogenesis of cellular material, increased nitric oxide production, reorganization of the actin cytoskeleton and more (Bauerfeld et al., 2012; Corradin et al., 1992; Covarrubias, Aksoylar, \& Horng, 2015; Kleveta et al., 2012). Altogether, the remodelling of activated innate immune cells drives enhanced microbial killing and the overall immune defense. 
The plasma membranes of phagocytic immune cells are decorated with an array of receptors that recognize key evolutionarily conserved structural signatures of bacterial and viral molecules such as flagellin, oxidized lipids and carbohydrate-protein repeats, called microbeassociated molecular patterns (MAMPs). These signature patterns are recognized by receptors known as pattern recognition receptors (PRRs), the Toll- like receptor (TLR) family is the most well-studied PRRs. Within this family of proteins, there are 10 members in humans and 13 in mice (O’Neill \& Bowie, 2007), some are plasma membrane receptors (i.e. TLR4, TLR5) and others are endosomal (i.e. TLR7, TLR8, TLR9) (Gay, Symmons, Gangloff, \& Bryant, 2014). Upon MAMP recognition via their extracellular leucine-rich-repeat (LRR) domain, TLR receptors can homo- or hetero-dimerize depending on the receptor type and ligand. Receptor dimerization leads to the interaction of the cytosolic TIR (Toll-IL-1 receptor) domains of each TLR and recruitment of downstream adaptor proteins, which can include MyD88 (Myeloid differentiation primary response protein 88), MAL (MyD88 adaptor-like), TRIF (TIR domain containing adaptor inducing IFN- $\beta$ ) and TRAM (TRIF-related adaptor molecule), depending on the MAMP-TLR receptor combination (reviewed here (O’Neill \& Bowie, 2007)). The MAMP-TLR receptor investigated in this thesis is of TLR4 and LPS.

Lipopolysaccharide (LPS) is recognized by TLR4 and can signal via MyD88-dependant or independent pathway (Figure 1.4). Depending on the pathway, LPS can initiate a plethora of responses. For example, the LPS induced MyD88-dependant pathway can lead to increased production and release of the pro-inflammatory cytokine TNF- $\alpha$, and upregulation of membrane trafficking to enhance particle uptake (Beg, Finco, Nantermet, \& Baldwin, 1993; Muta \& Takeshige, 2001; Shimazu et al., 1999; R M Steinman \& Swanson, 1995). Macrophages and DCs are referred to as "professional phagocytes" as they are constantly sampling their environment for 
invading material. Activated DCs and macrophages enhance their membrane trafficking rates thus promoting the process of phagocytosis to allow for antigen clearance.

\subsubsection{Phagocytosis and phagosome maturation}

Protozoa and certain cells within Metazoa have the ability to selectively internalize particles and degrade them intracellularly through a process known as phagocytosis (Figure 1.5a). In this process, plasma membrane remodelling promotes the engulfment of particles greater than $0.5 \mu \mathrm{m}$ in diameter into enclosed vacuoles called phagosomes, where the targets are degraded. Phagocytic cells express receptors, like lectins and scavenger receptors, that can recognize distinct molecular signatures endogenous to the target (Brubaker, Bonham, Zanoni, \& Kagan, 2015; Naik \& Harrison, 2013). Alternatively, phagocytes also express opsonic receptors that bind to opsonins deposited on the target, like complement and $\mathrm{Fc} \gamma$ receptors that bind to complement fragments and immunoglobulin G (IgG), respectively (Brubaker et al., 2015; Naik \& Harrison, 2013). Receptorligand clustering triggers changes in the composition of plasma membrane phosphoinositide lipids and the activation of small Rho GTPases, like RhoA, Rac1/2 and Cdc42, to stimulate the formation of actin-rich, membrane pseudopods, that surround the target, thereby forming a phagocytic cup (Herre et al., 2004; Swanson et al., 1999; Tomasevic et al., 2007).

The closure of the phagocytic cup results in the formation of a new phagosome, which undergoes maturation through a stepwise process that promotes the degradation of the internalized particle. The internal environment of the phagosome changes from neutral to acidic and acquires hydrolases through the fusion with endolysosomal compartments (early endosomes, late endosomes and lysosomes). In the initial stage of maturation, the nascent phagosome acquires the small GTPase Rab5, whose recruitment and activity is presumed (based on endosomal maturation) 
to be promoted through its cognate GEF Rabex-5, as well as through a positive feedback loop established by the Rab5/Rabaptin-5 complex (Horiuchi et al., 1997; Lippé, Miaczynska, Rybin, Runge, \& Zerial, 2001). Rab5 activity promotes early endosomal fusion through the recruitment of classical early endosome marker Early Endosome Autoantigen 1 (EEA1) and the p150/Vps34 complex (Christoforidis, Miaczynska, et al., 1999; Mu et al., 1995; Vieira, Botelho, \& Grinstein, 2002). In turn, the acquisition of Vps34 increases the localized synthesis of phosphatidylinositol3-phosphate [PtdIns(3)P], which then further enhances EEA1 recruitment (Christoforidis, Miaczynska, et al., 1999). EEA1 acts like a tether to coordinate and promote fusion between Rab5positive organelles, like early phagosomes and early endosomes (Christoforidis, McBride, Burgoyne, \& Zerial, 1999).

Following endosomal fusion, the early phagosome transitions into the phagolysosome. The Mon1-Ccz1 complex facilitates the loss of Rab5 and subsequent acquisition of Rab7, by displacing Rabex-5 and Rabaptin-5 (Kinchen \& Ravichandran, 2010; Poteryaev, Datta, Ackema, Zerial, \& Spang, 2010). The recruitment of active Rab7 primes the phagosome for fusion with the late endosomes and lysosomes through as yet unclear mechanisms. It is known that Rab7-interacting lysosomal protein (RILP) and oxysterol-binding protein-related protein 1L (ORP1L) work together to mediate microtubule-dependent trafficking of the phagosome towards the lysosome via the dynein-dynactin complex (Harrison, Bucci, Vieira, Schroer, \& Grinstein, 2003; Johansson et al., 2007). Moreover, tubular phagosome extensions are formed in a RILP-dependent manner and allow the phagosome to fuse with late endosomes and lysosomes (Harrison et al., 2003). The homotypic fusion and protein sorting (HOPS) complex promotes fusion through two Rab7interacting subunits Vps39 and Vps41, which tether two Rab7-positive compartments together to initiate and facilitate fusion via trans-SNARE complexes (Balderhaar \& Ungermann, 2013; 
Krämer \& Ungermann, 2011). The GTPase, ADP Ribosylation Factor Like 8B (Arl8B), also appears to coordinate HOPS complex association via kinesin-1 (Garg et al., 2011). In addition, the lysosomal $\mathrm{Ca}^{2+}$ channel, transient receptor potential mucolipin 1 (TRPML1), and phosphatidylinositol-3,5-bisphosphate $\left[\operatorname{Ptd} \operatorname{Ins}(3,5) \mathrm{P}_{2}\right]$ are also necessary for maturation (Dayam, Saric, Shilliday, \& Botelho, 2015; Dayam et al., 2017; G. H. E. Kim, Dayam, Prashar, Terebiznik, \& Botelho, 2014). The synthesis of PtdIns(3,5) $\mathrm{P}_{2}$ by the lipid kinase PIKfyve helps to deplete PtdIns(3)P from the early phagosome and promote its conversion to a late-stage phagosome (Hazeki et al., 2012; G. H. E. Kim et al., 2014). Moreover, PtdIns(3,5) $\mathrm{P}_{2}$ is thought to activate TRPML1, thus releasing $\mathrm{Ca}^{2+}$ from lysosomes and facilitating phagosome-lysosome fusion (Dayam et al., 2015).

As a consequence of the maturation process, the luminal content of the phagosome changes profoundly. The acquisition of vacuolar-ATPases establishes an acidic $\mathrm{pH}$ (4-4.5), which is optimal for the action of a number of hydrolytic enzymes, such as proteases, peptidases, phosphatases, nucleases, glycosidases, sulfatases and lipases (Appelqvist, Waster, Kagedal, \& Ollinger, 2013). The hydrolases function to degrade the target particle, which provides metabolites to the cell and produces antigen to prime the adaptive immune response through the process of antigen presentation. The stages of phagosome maturation have been investigated largely using spheroidal targets like latex beads or bacillus and coccus bacterium. However, in nature immune cells are challenged with an array of targets with disparate structures and morphologies.

\subsubsection{Differentially shaped targets of phagocytosis}

Bacteria have evolved a variety of mechanisms to escape the stages of phagocytosis (reviewed in: Lancaster, Ho, Hipolito, Botelho, \& Terebiznik, 2019). These mechanisms range from modifying 
their surface molecules to avoid recognition by immune cells, to hijacking the molecular mediators required for phagosome closure and maturation. For example, Salmonella can express different outer membrane O-antigens (components of LPS), to evade recognition by intestinal protozoans within the human body (Wildschutte, Wolfe, Tamewitz, \& Lawrence, 2004) and can modify their endotoxins to escape bacterial killing by the innate immune system (Matsuura, 2013). In addition to the molecular modifications, bacteria can also increase their size above the maximal uptake dimensions of their predator. This is seen with the bacterium Flectobacillus whom shifts from medium-sized bacteria to a filamentous form that is resistant to feeding by the protozoan Ochromonas (Corno \& Jürgens, 2006; Posch et al., 2001).

Moreover, bacterial filamentation is observed in pathogenic bacteria like Escherichia coli, Proteus mirabilis and Legionella pneumophila as their filamentous morphology poses a hurdle for phagocytosis by macrophages (Horvath et al., 2011; S. S. Justice, Hunstad, Seed, \& Hultgren, 2006; Prashar \& Terebiznik, 2015). Bacteria and fungi can also form clustered colonies challenging the phagocytic capacity of immune cells (Gregory J Cannon \& Swanson, 1992). However, immune cells have adapted mechanisms to deal with evolved tactics of these pathogens. For instance, macrophages can fuse together to form multi-nucleated cells with a larger capacity for phagocytic uptake and neutrophils can secrete antimicrobial factors through the process of degranulation to target extracellular microbes (Lacy, 2006; Milde et al., 2015). Apart from the differentially shaped microbial targets, immune cells are also challenged with a variety of abiotic particles in nature (Champion \& Mitragotri, 2006). These targets include natural and industrial materials like asbestos and glass fibers. Understanding how immune cells respond to these targets and how cell shape and size impacts phagocytic efficiency and uptake will help us better understand how researchers can design less harmful agents used in industrial settings or exploit 
these parameters to design better particles used for drug delivery (Champion \& Mitragotri, 2009; Lin, Hsu, Lo, Tsai, \& Hsiue, 2011; Mathaes, Winter, Besheer, \& Engert, 2014).

\subsubsection{Phagocytosis of tubular targets}

Upon immune cell recognition, target shape can also influence whether the cell and target correctly engage for phagocytosis to proceed. Champion \& Mitragotri (2006) showed that the local shape of a target and point of contact with the membrane of a macrophage determines whether phagocytosis will proceed or fail. Specifically, phagocytosis is only engaged when targets form a $45^{\circ}$ angle or less with the plasma membrane, as is observed with canonical spheroidal particles. Whereas, particles forming larger angles, stall membrane remodelling and uptake, so the target remains engaged at the plasma membrane. Using filamentous bacteria, Möller et al. (2012) also showed that filamentous targets that engage with the plasma membrane at one pole, form a small contact angle and are readily internalized, whereas, those that engage on their long-axis form a larger contact angle and engulfment does not proceed. This often occurs with longer targets like filamentous bacterium or fungi.

Target morphology does not only impact particle binding and engulfment, as target length directly impacts the duration and velocity of phagocytosis, as was shown using filamentous Escherichia coli (Möller et al., 2012) and Legionella pneumophila (L. pneumophila) (Prashar et al., 2013). The uptake of long filamentous microbes occurs gradually via the formation of a tubular phagocytic cup (tPC), where full particle engulfment and tubular phagosome closure can take up to 30 minutes. As the target is engulfed, F-actin strongly accumulates at the proximal region of the tPC forming a jacket-like structure (Gerisch et al., 2009; Heinsbroek et al., 2009; Maxson et al., 2018; Prashar et al., 2013). The actin jacket forms a diffusion barrier at the neck creating a membrane fence which helps to maintain and separate the molecular identity of the plasma 
membrane and the growing tPC (Golebiewska et al., 2011; Prashar et al., 2013). The decreased degradative lumen of the tPC and the delayed period of phagosome sealing likely promotes the survival of invading bacterium such as L. pneumophila which can maximize the number of microbial effectors injected into a host cell. Interestingly, in contrast to canonical phagocytosis, phagosome maturation occurs before full closure of the tPC, as demonstrated by Prashar et al. (2013) using PFA-killed filamentous L. pneumophila.

\subsubsection{Phagocytosis of filamentous Legionella pneumophilia}

L. pneumophila is a pathogenic bacterium that gives rise to the respiratory Legionnaires disease, a severe form of pneumonia. L. pneumophila thrives in fresh water environments in free living forms, biofilms and as an intracellular parasite. Infection by L. pneumophila occurs when the bacterium is in an aerosolized droplet inhaled through the alveolar mucosa. Upon invasion of macrophages, L. pneumophila delivers microbial effectors in the host cell to promote replication and survival. Its long filamentous morphology has been linked to its survival (Prashar et al., 2013). Due to its morphology, L. pneumophila (Lp) has been used as a model to study the maturation of tPCs, illustrating that the pathway deviates from the canonical spheroidal targets, giving rise to spatiotemporal alterations that affect maturation of the phagosome.

Using paraformaldehyde-killed Lp to block the activity of microbial factors, Prashar et al. (2013) demonstrated that even before tPC closure and scission from the plasma membrane, phagosomes begin to mature opposed to the canonical pathway (Figure 1.5b). Specifically, tPCs undergo similar actin remodelling, where $\operatorname{PtdIns}(4,5) \mathrm{P}_{2}$ is locally produced at the proximal portion of the tPCs but not at the base, consistent with its the biphasic appearance in the canonical pathway. Interstingly, after 5 mins of internalization, the early endosomsal markers EEA1 and probe for 
PtdIns(3)P, 2FYVE-GFP, localize to the tip. After 20 minutes, the lysosomal markers Rab7 and lysosome-associated membrane protein 1 (LAMP1) are also recruited, even before phagosome closure (Prashar et al., 2013). Contrary to the canonical pathway, as early and late endosomal compartments are recruited to the growing tPC, the luminal environment does not become degradative and acidic despite recruiting V-ATPases, instead this only occurs after phagosome closure. Likely the actin-jacket creates a low-molecular weight diffusion barrier, allowing for the passage of small molecular weight proteins, like endosomal cathepsins, and ions like protons and calcium to leak out into the extracellular milieu. As a result, the luminal properties of the most proximal region of the tPC are less acidic and degradative, and the microbicidal capacity of the phagosome is compromised (Maxson et al., 2018; Prashar et al., 2013). This tubular phagosome model has provided clear evidence that tubular morphology can impact phagocytic cup formation and timing of maturation (Figure 1.5b).

These data suggest that other molecular switches are likely at play to coordinate the turnover of phagosomal markers and complete phagosome maturation. Possibly more time is needed for proper acidification of the phagosome due to the diffusion barrier. Moreover, there could be a luminal gradient associated with the length of filaments and the stages of phagocytosis. By using PFA-killed Lp and exploiting the spatiotemporal resolution of the $\mathrm{tPC}$, it may be possible decipher the intricate mechanisms of tubular phagosome maturation and its regulators.

\subsubsection{Antigen presentation and processing}

Following efficient phagocytosis and break-down of antigenic material, the innate immune system relays the immune signal to the adaptive immune system through antigen presentation (Figure 1.6). Macrophages and DCs are also known as professional antigen presenting cells (APCs) and can 
relay inflammatory signals to the adaptive immune system. Both cells synthesize major histocompatibility complex type II (MHCII) molecules that are composed of $\alpha$ and $\beta$ heterodimers and contain a highly polymorphic region known as the antigen-binding groove, which binds to an array of antigens (Blum, Wearsch, \& Cresswell, 2013; Watts, 1997). Newly synthesized MHCII is trafficked to a compartment known as the lysosome-related MHCII compartment (MIIC). To avoid premature antigen loading during MHCII production and trafficking, the peptide binding groove is blocked by the invariant chain (Roche \& Cresswell, 1990; Stumptner \& Benaroch, 1997). When exposed to the lysosomal lumen, $\mathrm{pH}$-sensitive hydrolases cleave the invariant chain, leaving the small CLIP (Class II associated invariant chain peptide) fragment within the groove. Next, the MHCII like molecule, HLA-DM (human leukocyte antigen- DM) catalyzes the release of CLIP from MHCII and the antigen binding groove (Kropshofer, Arndt, Moldenhauer, Hämmerling, \& Vogt, 1997). The empty groove is quickly occupied by a small digested antigen fragment (15 to 24 amino acids in length) forming the antigen-MHCII complex.

Upon antigen loading in the lysosome, mature DCs deliver large amounts of antigenMHCII complexes to their plasma membrane for recognition by $\mathrm{CD}^{+} \mathrm{T}$ helper cells (Cella, Engering, Pinet, Pieters, \& Lanzavecchia, 1997). Trafficking of this complex is known to be regulated by extension and tubulation of the MIIC compartment (Marianne Boes et al., 2002; Chow, Toomre, Garrett, \& Mellman, 2002; Vyas et al., 2007). Once displayed at the surface of the plasma membrane, the antigen-MHCII complex is recognized by CD4+ T helper cells, directly interacting with the T-cell receptor and the co-receptor CD4 (Askew, Gatewood, Olivas, Havenith, \& Walker, 1995; Banchereau \& Steinman, 1998). Antigen presentation is critical for T-cell activation and priming an adaptive immune response. 


\section{$1.5 \quad \underline{\text { Lysosomes }}$}

The antimicrobial activity of phagocytic immune cells is attributed to the function of the acidic and degradative organelle called the lysosome. Lysosomes are small globular organelles $(<0.5$ microns) and can occasionally appear tubular (Boes et al., 2003; Swanson, Yirinec, \& Silverstein, 1985). Lysosomal size and number can range depending on cell-type, where lysosomal diameter can exceed several microns depending on the accumulation of undigested material (Steinman, Brodie, \& Cohn, 1976). Lysosomes are enclosed in a lipid bilayer and decorated with membrane proteins that are heavily $\mathrm{N}$-glycosylated at their luminal end, yielding to about $60 \%$ of the total protein mass (E. L. Eskelinen, Tanaka, \& Saftig, 2003; Neiss, 1984). It is thought that lysosomal membrane protein glycosylation forms a protective layer called a glycocalyx, which helps to preserve the integrity of the lysosomal membrane and its proteins from the degradative lumen, however, it likely also regulates luminal lysosomal signalling and cargo recognition.

Fifty-percent of the membrane protein population, is made up of two major groups of proteins, the lysosome-associated membrane protein 1 and 2 (LAMP1/2) and lysosomal integral membrane protein 2 (LIMP2) (Eskelinen, 2006; Hunziker, Simmen, \& Höning, 1996). As previously described, LAMP2 is important for chaperone mediated autophagy whereas, LAMP1 is involved in plasma membrane binding to the cellular substratum and is also hypothesized to interact with microtubule motors (Andrews, 2002; Jensen, Aaberg-Jessen, Christensen, \& Kristensen, 2013). LIMP2 is essential for sorting and targeting of de novo lysosomal enzymes (Reczek et al., 2007). Alongside those receptors, other integral membrane proteins are important for lysosomal cargo transport such as Niemann-Pick disease type C1 (NPC1) and SLC38A9 which facilitate the transport of lipids and amine-substrates, and the amino acid arginine, respectively. Mutations or absence of some of these proteins can lead to deleterious diseases such as Neimann 
Pick Disease (Millat et al., 2001) and embryonic lethality (Eskelinen, 2006), thus illustrating the importance of functional lysosomes. Apart from the role of structural membrane proteins in maintaining lysosomal homeostasis, luminal properties are also vital for its function.

Lysosomes contain an acidic lumen $(\mathrm{pH} 4.5-5.5)$ that is established by the action of vacuolar-type $\mathrm{H}^{+}$-ATPase (V-ATPase) (Ohkuma, et al., 1982; 1983). The V-ATPase actively transports protons from the cytosol into the lysosomal lumen. Concurrently, counter-ion efflux such as potassium, or influx with chlorine is predicted to dissipate the voltage difference generated upon proton transport (Graves, Curran, Smith, \& Mindell, 2008; Xiong \& Zhu, 2016). Apart from the role of protons and other counter-ions, calcium has been shown to play an important role in lysosome homeostasis and signalling. Lysosomal calcium levels can be as high as 400-600 $\mu \mathrm{M}$ (Christensen, Myers, \& Swanson, 2002; Seto, Bellen, \& Lloyd, 2002) similar to the cell's calcium sink, the endoplasmic reticulum (100-800 $\mu \mathrm{M})$ (Bygrave \& Benedetti, 1996). Although the regulation of lysosomal calcium is not fully understood, the ion-channel mucolipin TRP channel subfamily (TRPML1) (Yamaguchi et al., 2011) plays an important role in several pathways, and a candidate calcium pump has also been reported (Adachi, Arai, \& Ohkuma, 1996; Ezaki, Himeno, \& Kato, 1992; Melchionda, Pittman, Mayor, \& Patel, 2016) but not yet characterized. Lysosomal calcium is important for phagosome maturation, lysosome motility and structure and bacterial clearance (Dayam et al., 2015; X. Li et al., 2016). Deficits in lysosomal calcium have been linked to impaired bacterial killing and may contribute to lysosomal storage disease like Mucolipidosis type IV (Lloyd-Evans \& Platt, 2011). Altogether the luminal ion content of the lysosome is optimal for the degradation of macromolecules by activating the enzymatic function of $\mathrm{pH}$-sensitive hydrolytic enzymes (Coffey \& De Duve, 1968). 
The lysosomal protein catalysts that breakdown macromolecules are called hydrolases, and function optimally at an acidic $\mathrm{pH}$ (Appelmans, Wattiaux, \& De Duve, 1955). Approximately 60 resident hydrolases that include proteases, peptidases, phosphatases, nucleases, glycosidases, sulphatases and lipases are responsible for target-specific degradation of molecules, and collectively function to breakdown complex macromolecules into small peptides of 15-25 amino acids long (Appelqvist et al., 2013). A large family of proteases called cathepsins, are specific to the lysosome and facilitate the breakdown of proteins (Rossi, Deveraux, Turk, \& Sali, 2004).

The lysosome serves as a convergence point for all the major macromolecule degradative pathways: endocytosis, phagocytosis and autophagy, as previously discussed (Figure 1.1). Additionally, lysosomes are known to function in a variety of processes such as membrane repair (Reddy, Caler, \& Andrews, 2001), bone reabsorption by osteoclasts (Lacombe, Karsenty, \& Ferron, 2013), promote secretion needed for cell migration (Bretou et al., 2017) and have been implicated in the progression of metastatic cancer (Fennelly \& Amaravadi, 2017).

\subsubsection{Lysosome function in immunity}

Lysosomes are kingpins in macrophages and DCs, driving an array of immune signalling pathways that are imperative for immunity including macropinocytosis, phagocytosis and antigen presentation (Figure 1.1). As previously described, lysosomes are the degradative hub in these cells, driving the killing and clearance of invading particles like bacteria and viruses (Deretic \& Levine, 2018; Fairn \& Grinstein, 2012; M. Gray \& Botelho, 2017; Korolchuk et al., 2011; Levin, Grinstein, \& Canton, 2016; Pauwels, Trost, Beyaert, \& Hoffmann, 2017). Dendritic cells, in particular, must orchestrate lysosome degradative capacity to preserve antigen peptides for antigen presentation (Pauwels et al., 2017; Savina \& Amigorena, 2007). In addition to their degradative 
role, they are also important for lymphocyte function, control cellular migration and can coordinate cytokine signalling.

Lysosome positioning and function regulate cytotoxic CD8 ${ }^{+} \mathrm{T}$ lymphocytes (CTLs) and natural killer (NK) cells' ability to kill tumor or virally-infected cells. Upon immune synapse formation between a CTL and a target cell, lysosomal-related organelles called lytic granules, are secreted, releasing their contents onto the target cell. Lytic granules contain cytotoxic mediators in addition to lysosomal luminal proteins like LAMP1 and hydrolases (Blott \& Griffiths, 2002; Dell'Angelica, Mullins, Caplan, \& Bonifacino, 2000). Moreover, lytic granule targeting to the periphery of CTLs and NKs prior to release depends on the well-established microtubule dependent lysosome-positioning machinery Rab7-RILP-dynein and Arl8-SKIP-kinesin-1 ensembles, (Daniele et al., 2011; Tuli et al., 2013). Interference of these microtubule motor complexes reduces the ability of CTLs and NKs to kill their targets.

Lysosomal function has also been associated with the activation of the Nod-like receptor3 (NLRP3) inflammasome upon infection with Mycobacterium tuberculosis (M. tuberculosis). The NLRP3 inflammasome is a cytosolic receptor that recognizes DAMPs and MAMPs that have evaded the phagosome, akin to TLR activation at the plasma membrane. Activation of the multisubunit complex is multifaceted and requires priming, often by TLR controlled NFK-B signalling. Secondary to priming, an array of microbial factors further activates NLRP3, such as the earlysecreted antigen (ESAT-6) which is released from M. tuberculosis facilitating its escape from the phagosome (Amaral et al., 2018). The adaptor protein ASC (apoptosis-associated speck-like protein containing a carboxy-terminal CARD), is then recruited, which is required for caspase-1 recruitment and activation. Recruitment and polymerization of these mediators forms a giant multisubunit complex culminating in the formation of the NLRP3-inflammasome complex (Guo, 
Callaway, \& Ting, 2015). Once assembled, the NLRP3-inflammasome complex activates the maturation of pro-IL-1 $\beta$ and release of its mature form, in a caspase-1-dependent manner. Interestingly, Amaral et al. (2018) demonstrated that increased cytosolic ESAT-6 levels induced by M. tuberculosis overexpression led to lysosomal membrane permeabilization and leakage of lysosomal cathepsin B. Increased cytosolic cathepsin B was required for sufficient NLRP3 activation and amplification of IL- $1 \beta$ to exacerbate inflammation. Likely in addition to cathepsin $\mathrm{B}$, other lysosomal cathepsins may also control inflammasome complex formation, imperative for immunity.

Lysosomal function has also been implicated in phagocyte cell migration. Bretou et al. (2017) demonstrated that when dendritic cells are exposed to LPS, lysosomal calcium is released by TRPML1 into the cytosol remodelling the actin cytoskeleton. First, there is a burst of filamentous actin structures at the trailing end of the cell and decreased at the leading edge, secondly, activating actin-based motor myosin II. Together, TRPML1-mediated lysosomal calcium release promotes localized actin reorganization and directional migration. Moreover, the lysosome has also been linked to cancer cell metastasis. Where, lysosomal exocytosis results in the localized release of acid hydrolases into the extracellular space and insertion of late endosomal/lysosomal membrane proteins onto the plasma membrane (Mohamed \& Sloane, 2006; Monteiro et al., 2013; Poincloux et al., 2009). Secretion of acid hydrolases promotes ECM breakdown facilitating cell migration and invasion, whereas plasma membrane lysosomal proteins help to form attachments to the ECM. Although not yet elucidated, it is possible that a similar mechanism is being used by phagocytes while they surveil tissues for infection.

Additionally, lysosomes can negatively and positively regulate cytokine release from activated phagocytes. Pro-inflammatory and anti-inflammatory cytokines are discharged into the 
extracellular space to prime nearby immune cells promoting or downregulating inflammation, respectively. Similar to the role the lysosome plays in promoting IL-1 $\beta$ production following inflammasome activation, IL- $1 \beta$ release is controlled by secretory lysosomes. Cytosolic calcium release mobilizes secretory lysosomes to the plasma membrane discharging IL-1 $\beta$ and other lysosomal components into the extracellular space (Andrei et al., 2013, 2004). The lysosome is also linked to the negative regulation of cytokine signalling. For example, in the presence of antiinflammatory signals, cytokine receptors such as TGF- $\beta$ receptor I and gp130 (Hao et al., 2011; Radtke et al., 2010) are trafficked from the plasma membrane to the lysosome for degradation, downregulating TGF- $\beta$ and IL-6 signalling. In addition to receptor downregulation, the lysosome mediates the release of the anti-inflammatory protein, flightless. Flightless blocks cytokine signalling and release by preventing caspase-1 dependent maturation of pro-cytokines, as it behaves as a pseudo-substrate for caspase-1 hindering its catalytic activity (Lei et al., 2012; J. Li, Yin, \& Yuan, 2008).

Evidently the lysosome is linked to and regulates an array of immune responses in lymphocytes and phagocytic cells. Apart from their importance for immune cell function, we are only beginning to appreciate that lysosomes respond and adapt to infection and telltale signals of infection.

\subsubsection{Lysosome adaptation}

Lysosomes have classically been viewed as a static and homogeneous population of acidic and degradative organelles. However, this perception is giving way to a more sophisticated view where lysosomes are heterogeneous, dynamic and adaptable (Martina \& Puertollano, 2017; Settembre \& Ballabio, 2014; Xu \& Ren, 2015). For example, lysosome number, which can differ 
greatly depending on cell-type, is reduced upon starvation (Xu \& Ren, 2015; Li Yu et al., 2010). Additionally, a single cell may have some lysosomes positioned at the cell periphery and others at the perinuclear region (Heuser, 1989; X. Li et al., 2016; Pu et al., 2015) depending on cellular state and stress. In non-polarized cells, lysosomes are clustered towards the microtubule organizing center (Jongsma et al., 2016), whereas, in polarized cells like neurons, lysosomes are distributed within the soma, across axons and dendrites.

Lysosomes are dynamic and display a "stop-and-go" motion, suggesting that motility can be regulated. Lysosomes can be forced to displace all together to the cell periphery or centrally upon alkalinization or acidification of the cytosolic $\mathrm{pH}$, respectively (Heuser, 1989). Moreover, cellular stress and signalling can control lysosomal distribution, where starvation (Korolchuk et al., 2011), drug-induced apoptosis (Le Yu et al., 2016) and expression of pathogenic mutant forms of huntingtin (Erie, Sacino, Houle, Lu, \& Wei, 2015) cause perinuclear clustering and impact cellular function. Differential positioning of the lysosome has also been shown to have functional implications, where peripheral lysosomes are more alkaline than perinuclear lysosomes (D. E. Johnson, Ostrowski, Jaumouillé, \& Grinstein, 2016). This distribution is thought to be a result of decreased access to newly synthesized proteins necessary for lysosomal function. Together these insights provide evidence that lysosomes are not static and can respond and adapt to cellular cues.

Collectively, these parameters are under the control of a complex regulatory network that can sense the various needs of the cell and remodel and adapt lysosomes to suit those needs. Lysosomes are bona fide signalling platforms that sense, integrate and inform the cell about its nutrient levels through coordinating the activities of the antagonistic kinase hubs mTORCland AMPK (Carroll \& Dunlop, 2017; Inpanathan \& Botelho, 2019; Lim \& Zoncu, 2016; Sardiello et al., 2009; Settembre et al., 2012; Zhang et al., 2014). Where upon recruitment to the lysosome, 
AMPK activates the TSC1/2 complex, which promotes GTP hydrolysis by the Rheb GTPase, therein arresting Rheb, an mTORC1 stimulant (Inoki et al., 2003; Lim \& Zoncu, 2016; Long, Lin, Ortiz-Vega, Yonezawa, \& Avruch, 2005; Zhang et al., 2014). mTORC1 and AMPK then govern the balance of anabolism and catabolism to serve the cell's requirements, which includes adapting lysosome function by regulating TFEB and TFE3, as previously described (Martina \& Puertollano, 2017; Roczniak-Ferguson et al., 2012; Settembre et al., 2011, 2012) . In the context of immunity, mTORC1 has been shown to coordinate lysosomal biogenesis to boost bacterial killing, phagosome maturation and promote clearance (Gray et al., 2016; Pastore et al., 2016; Sardiello et al., 2009). 


\subsection{Lysosome tubulation}

Lysosomal adaptation can be appreciated to boost immunity and bolster lysosomal function. Interestingly, lysosome morphology can be quite heterogenous, ranging from globular structures with a diameter of $0.5-1 \mu \mathrm{m}$, to a highly tubular network in activated macrophages and dendritic cells (DCs) (Heuser, 1989; Medina et al., 2015; Pu, Guardia, Keren-Kaplan, \& Bonifacino, 2016; Joel Swanson, Bushnell, \& Silverstein, 1987).

Resting macrophages and DCs are enriched in spheroidal lysosomes of about 0.5-1 $\mu \mathrm{m}$ in diameter (Appelqvist et al., 2013; Lullmann-Rauch, 2005). However, stimulation of these cells with several agonists causes a dramatic reorganization of lysosomes into a tubular network. In macrophages, lysosomes labelled with pinocytic fluid-phase markers undergo tubulation upon exposure to phorbol 12-myristate 13-acetate (PMA) (J. A. Swanson et al., 1985; J Swanson, Burke, \& Silverstein, 1987) or to lipopolysaccharides (LPS) (X. Li et al., 2016; Mrakovic, Kay, Furuya, Brumell, \& Botelho, 2012; Saric et al., 2016). In DCs, MHC-II compartments, the lysosomerelated organelle, undergo extensive tubulation during DC maturation with LPS (Barois, De SaintVis, Lebecque, Geuze, \& Kleijmeer, 2002; Bertho et al., 2003; Chow et al., 2002; Kleijmeer et al., 2001; Nakamura et al., 2014), during immune synapse formation (Boes et al., 2002; Chow et al., 2002), and upon fungal phagocytosis (Vyas et al., 2007). These tubules are positive for the classical lysosomal markers LAMP1 and CD63, and can be marked with the acidotropic dye Lysotracker (Vyas et al., 2007). Lysosome tubules are likely heterogeneous depending on cell type and activation state.

While the above represents a near complete transformation of lysosomes into a tubular network, there is also localized lysosome tubulation. For example, lysosome tubules emanate from and towards phagosomes during phagosome maturation and during autophagosome-lysosome 
reformation (Harrison et al., 2003; Mantegazza et al., 2014; Sun-Wada, Tabata, Kawamura, Aoyama, \& Wada, 2009; Li Yu et al., 2010). Moreover, lysosome tubulation has also been observed in resting macrophages cultured from Beige mice, an orthologous disorder for human Chediak-Higashi syndrome. Resting lysosomes from beigej cells were significantly more tubular than wild-type counterpart and was attributed to decreased lysosomal fissioning rates in these cells (Durchfort et al., 2012). In addition to their manifestation in immune cells, lysosomal tubulation has also been observed in cardiac smooth muscle cells, Drosophila muscle cells and exocrine acinar cells, contributing to the degradative and secretory capacities of these cell-types (Bendayan \& Gisiger, 2001; A. E. Johnson, Shu, Hauswirth, Tong, \& Davis, 2015; Oliver, 1983; Robinson, 1986). Clearly understanding how lysosome tubulation is regulated is critical to understanding their seemingly important functional roles.

\subsubsection{Pathways leading to lysosome tubulation}

Exceptionally little is known about the signalling pathways that allow triggers such as LPS to communicate and comprehensively remodel lysosome morphology in macrophages and DCs. It was only in 2007 when Vyas et. al described the necessity for TLR adaptor protein MyD88, in response to LPS, almost two decades after their initial discovery (Joel Swanson et al., 1987). LPSinduced lysosome tubulation signals through Toll-like receptor-4 (TLR4) and requires MyD88 since MyD88-defective DCs and macrophages fail to tubulate lysosomes in response to LPS (Boes et al., 2003; Saric et al., 2016; Vyas et al., 2007). Additionally, other receptors have also been shown to modulate lysosome function since phagocytosis of the fungal pathogen Cryptococcus neoformans also induces tubulation. C. neoformans engages both TLR2 and TLR4, but lysosomal 
tubulation occurred even in MyD88 ${ }^{-/-}$DCs (Vyas et al., 2007), suggesting that many stimuli may feed into a common signalling network to induce lysosomal tubulation.

Focusing on LPS-induced lysosome tubulation, our group showed that lysosome tubulation requires the phosphatidylinositol-3-kinase-Akt-mTOR signalling axis independent of autophagy (Saric et al., 2016) (Figure 1.7). Inhibition of MyD88 and any component of the PI3K-AKT-mTOR axis, pathway greatly abates lysosomal tubulation, while inhibition of other MyD88 downstream effectors such as IRAK1/4 and TBK had no effect on lysosomal tubulation. As previously described, mTOR activity is also required for the initiation of lysosomal tubulation during the specialized process of autolysosome reformation (Munson et al., 2015; Li Yu et al., 2010), therefore, mTOR is a likely candidate to serve as a convergence point for modulating lysosomal tubulation and remodelling.

How might mTOR activity induce tubulation upon LPS exposure? We showed that LPS treatment increases the levels of Arl8b on lysosomes in an mTOR-dependent manner (Saric et al., 2016). Interestingly, the Arl $8 \mathrm{~b}$ GTPase has emerged as a lysosome master regulator that rivals the canonical Rab7 GTPase, controlling a variety of parameters including lysosome positioning, trafficking and tubulation itself(Garg et al., 2011; Hofmann, 2006; Michelet et al., 2015; Mrakovic et al., 2012). Understanding the molecular machinery required for the extension of lysosomal membranes is important for us to better understand the function of this remodelling process.

\subsubsection{Motors and molecular machinery driving lysosome tubulation}

The molecular machinery required for lysosome tubulation has been increasingly recognized over the last few decades (Figure 1.7). Lysosome tubulation depends on an intact microtubule network and the antagonistic motor activities of kinesin-1 and dynein (Boes et al., 2002, 2003; Hollenbeck 
\& Swanson, 1990; Li et al., 2016; Mrakovic et al., 2012; Swanson et al., 1987; Vyas et al., 2007). A reasonable model is that microtubules provide a physical template to extend lysosomes via the opposing forces generated by kinesin and dynein. These motors are linked to lysosomes via the lysosomal Rab7 and Arl8b GTPases. Specifically, the Rab7 GTPase links lysosomes to dynein and kinesin-1 via RILP and FYCO1, respectively (Jordens et al., 2001; Pankiv et al., 2010). In comparison, the Arl8b GTPase recruits kinesin-1 via SKIP to lysosomes, which catalyzes lysosome movement to the periphery (Rosa-Ferreira \& Munro, 2011). Strikingly, our lab showed that LPS-induced lysosome tubulation in macrophages requires all these factors (Mrakovic et al., 2012).

How Rab7 and Arl8b GTPases and their effectors coordinate dynein and kinesin-1 to productively generate lysosome tubules is an enigma. One possibility is that the dynein branch is stimulated during LPS exposure by selective $\mathrm{Ca}^{2+}$ efflux from lysosomes via the lysosomal calcium channel TRPML1 (Li et al., 2016). The release of $\mathrm{Ca}^{2+}$ then activates ALG2, a positive modulator of dynein-dynactin complexes (Li et al., 2016). In comparison, the augmented levels of Arl8b GTPase on lysosomes may boost kinesin activity to pull lysosomes to the cell periphery, generating tension (Saric et al., 2016). The mTOR-dependent boost in Arl8b membrane deposition may be regulated by the multi-subunit complex BORC1 (BLOC-1 related complex) (Pu et al., 2015). BORC1 is important for Arl8b to recruit kinesin motors, including kinesin-1 and kinesin3, to move lysosomes to the cell periphery (Guardia, Farías, Jia, Pu, \& Bonifacino, 2016; Pu et al., 2016). However, the Ragulator complex of mTOR also negatively modulates BORC1 and Arl8b activity during amino acid starvation (Filipek et al., 2017; Pu, Keren-Kaplan, \& Bonifacino, 2017), suggesting that the mTOR machinery differentially governs Arl8b depending on cell-context. 
Overall, given that lysosome tubules grow outward and track with EB1 (plus-TIP endbinding protein 1), a positive end microtubule tracking protein, Arl8b-kinesin-1 may be relatively more active than dynein in LPS-exposed cells (M. Boes et al., 2003; Chow et al., 2002; Vyas et al., 2007). Since dynein is required for tubulation, we can speculate that dynein may act as an anchor, while kinesin aids to extend the lysosomal membrane. However, lysosome stretching alone cannot explain how lysosomal length increase as tubules can reach to greater than $10 \mu \mathrm{m}$ in length. Therefore, there must be a balance between motor activity and coordinated membrane influx and/or lysosome coalescence to enlarge lysosomes to produce lengthy lysosome tubules; indeed, enlarged lysosomes are observed in LPS-treated macrophages disrupted for motor activity (Mrakovic et al., 2012).

\subsubsection{Functional implications of lysosome tubulation}

The function of lysosomal tubulation remains elusive. In macrophages stimulated with PMA, lysosome tubulation is associated with increased retention of fluid-phase endocytosis, which suggests an expanded lysosome volume (Swanson, Burke, \& Silverstein, 1987). In DCs, lysosome tubules are proposed to help deliver peptide-MHC-II to the plasma membrane, including immune synapses (Barois et al., 2002; Marianne Boes et al., 2002; Chow et al., 2002; Saric et al., 2016). Specifically, when dendritic cells are stimulated with LPS, MIIC compartments, become tubular and are required for efficient antigen presentation (Chow et al., 2002). Furthermore, this type of MIIC tubulation was also reported when DCs form an immunological synapse with T-cells (Bertho et al., 2003; Boes et al., 2003; Boes et al., 2002), illustrating that MIIC tubulation functions to enhance antigen delivery to the immunological synapse during antigen presentation. Tubulation may also facilitate fusion between phagosomes and lysosomes and mediate exchange between 
phagosomes to help homogenize antigenic content (Harrison et al., 2003; Mantegazza et al., 2014; Stephen et al., 2007; Sun-Wada et al., 2009).

More recently, Nakamura et al (2014) showed that endo-lysosomal tubulation in macrophages and DCs may also serve as signalling platforms for triggering adaptive immune responses. Extensive tubulation was observed in cells overexpressing two immune cell $\mathrm{H}^{+}$/oligopeptide symporters, SLC15A3 and SLC15A4. Interestingly, LPS-induced tubulation in BMDCs was blocked in slc15a3-/- cells, but rescued upon retroviral overexpression of SLC15A3GFP (Nakamura et al., 2014). These channels were required for the transport of bacterial compound muramyl dipeptide (MDP) and NOD2-dependent responses. It was also determined that tubule generation was required for proper recruitment of NOD2 signalling effectors and downstream immune signalling. The authors hypothesized tubulation enhances the surface area of lysosomes to boost MDP escape and detection by lysosome-associated NOD2.

Aside from the role of lysosome tubulation in immunity, it has also been implicated in the degradative and trafficking capacity of other cells. Lysosomal tubulation has been observed in cultured smooth muscle cells as early as the 1980's (Robinson, 1986). Muscle cells are unique in that basal autophagy is required to maintain muscle mass and homeostasis (Masiero et al., 2009). It is predicted that the constitutive levels of autophagy is likely related to the large energy demand of muscles and that they serve as a source of metabolic energy for other organs (Sandri, 2010). Interestingly, within the muscle sarcoplasm, lysosomes form a dynamic tubular network. Johnson et al. (2015) predicted that lysosome tubulation facilitates the local recycling of nutrients to meet local autophagic demands. Moreover, they found that tubulation was dependent on the AAAATPase Valosin-containing protein (VCP) (Johnson et al., 2015). Mutations in VCP have also been associated with a number of neurological and muscle degeneration diseases. Lysosomal 
tubulation has also been observed in exocrine acinar cells. Although not fully understood, it is predicted that tubulation of the lysosomal population helps to facilitate secretion by these cells through tubular tracks (Bendayan \& Gisiger, 2001; Oliver, 1983).

Altogether these findings support the importance of lysosomal tubulation in the context of immunity and other cellular responses. However, there are still many questions surrounding the function and context leading to lysosome tubulation.

\subsubsection{Outstanding questions}

Lysosome tubulation has been increasingly investigated in the context of immunity, yet it still remains unclear how other cell-types may utilize lysosomal remodelling to boost cell survival and function. By understanding the differential cellular contexts that induce lysosome tubulation, it may help us understand the possible cell-types and contexts that utilize this phenomenon.

Additionally, while lysosome tubules accumulate acidotrophic dyes, it is unclear if tubular and punctate lysosomes have the same luminal $\mathrm{pH}$, cargo transport rates, and degradative power. Understanding how tubular and punctate lysosomes compare in luminal and functional properties may shed light on how cells may differentially regulate the population to counterbalance cellular needs and extrinsic factors. Moreover, it is also unknown how lysosome tubules compare between macrophages and DCs, and in what other context do lysosome tubules form. For example, one interesting possibility is that lysosome tubulation may help explain changes in lysosome acidification during DC maturation since lysosomes in resting DCs are generally less-acidic than those in activated DCs are, a process that affects antigen survival (Delamarre, Pack, Chang, Mellman, \& Trombetta, 2005; Trombetta, Ebersold, Garrett, Pypaert, \& Mellman, 2003). Moreover, reactive oxygen species is another unexplored area in the context of lysosome 
tubulation that may impact lysosome properties. To illustrate, the NADPH oxidase prevents acidification of phagosomes in DCs even after fusion with lysosomes, affecting antigen processing (Jancic et al., 2007; Mantegazza et al., 2008; Savina et al., 2006). Overall, the function and properties of lysosome tubules remains a nebulous area of lysosome and innate immune biology. 


\subsection{Project goals and rationale}

The cells of our immune system have evolved elaborate and coordinated processes to identify, target and clear our body of invading particles. Cellular signalling pathways are tightly regulated and adapt in response to the signalling molecule present and the cellular state and functional demand of the cell. By understanding which molecular machines control these processes and how they are adapted, we may better understand how we can exploit these processes to enhance our immune responses. For my Ph.D. thesis I have two main projects (outlined in sections 1.7.1 and 1.7.2) that investigate different aspects of innate cell immunity, but together elucidate two novel mechanisms that control organelle identity and adaptation in immune cells.

\subsubsection{Maturation of tubular phagosomes}

When challenged with foreign particles immune cells elicit a variety of responses. One of these is engaging in phagocytosis, whereby the internalized particle undergoes maturation within the phagosome that progressively acidifies and becomes degradative upon fusion with the lysosome. In the canonical pathway, acidification precedes and primes the phagosome for fusion. Interestingly, upon internalization of tubular targets, recruitment of late endosomal markers precedes acidification. Due to the temporal and spatial resolution of the long phagocytic cup formed upon internalization of Legionella pneumophila, we hypothesize that the tubular phagocytic cup (tPC) can be used as a model to study phagosome maturation. Furthermore, we hypothesize that tPC luminal properties dictate maturation. This project was completed in collaboration with Amriya Naufer from University of Toronto. Using the two research aims listed next, I investigated the role of $\mathrm{pH}$ in tubular phagocytic cup maturation. 
1) Investigate membrane identity dynamics on growing tPCs

2) Uncover the mechanisms controlling membrane identity on tPCs

\subsubsection{Lysosome adaptation and remodelling in activated phagocytes}

Lysosomes are important organelles in several cell-types and cellular states. Lysosomes are linked to a variety of lysosomal storage diseases, neurological diseases, cancer and more (Fennelly \& Amaravadi, 2017; Platt, Boland, \& van der Spoel, 2012; Vellodi, 2005). In the context of immunity, lysosomes are kingpins in macrophages and dendritic cells. They serve as the degradative centre for the clearance of invading particles through the MIIC complex, and cellular debris via autophagy. Lysosomes are also important for relaying information to the adaptive immune system and play a direct role in T-cell mediated immunity. It is essential to understand how this organelle responds and adapts to various immune stimuli when considering its regulation and role in immunity.

Lysosomal tubulation evidently plays important roles in the context of immunity. Though some functional insights and the molecular machines that coordinate tubulation have begun to be identified, many remain elusive. Saric et al. (2016) showed that stimulation of dendritic cells and macrophages with LPS for 2 hours results in striking morphological changes. Interestingly, the majority of publications investigating lysosome remodelling and activation of immune cells characterize long-term activation, from 6 hours up to 48 hours (e.g. Pastore et al., 2016). My main research goal is to elucidate the overall significance and function of lysosome remodelling following acute activation of phagocytes, and to shed light on the molecular mediators that control this phenomenon. I hypothesize that acute activation of phagocytes remodels the lysosomal population to increase lysosomal volume and holding capacity. Furthermore, I hypothesize that 
lysosomal remodelling increases the ability of phagocytes to activate adaptive immunity. To address these hypotheses, I set out the following research aims and objectives.

Aim 1: To elucidate the molecular mechanisms that govern lysosome remodelling in activated phagocytes and its impact on boosting immunity

a) Determine if acute activation remodels the lysosomal population and increases biogenesis

b) Characterize the molecular machinery that boosts lysosome volume and biogenesis

c) Determined if lysosomal remodelling boosts antigen presentation and T-cell activation

Aim 2: To understand the role of lysosome remodelling during vaccination to boost immunity

a) Determine if adjuvants currently used in vaccines activate lysosome remodelling

b) Determine if adjuvant-induced lysosome remodelling boosts antigen presentation and Tcell activation 
$1.8 \quad$ Figures 
Figure 1.1

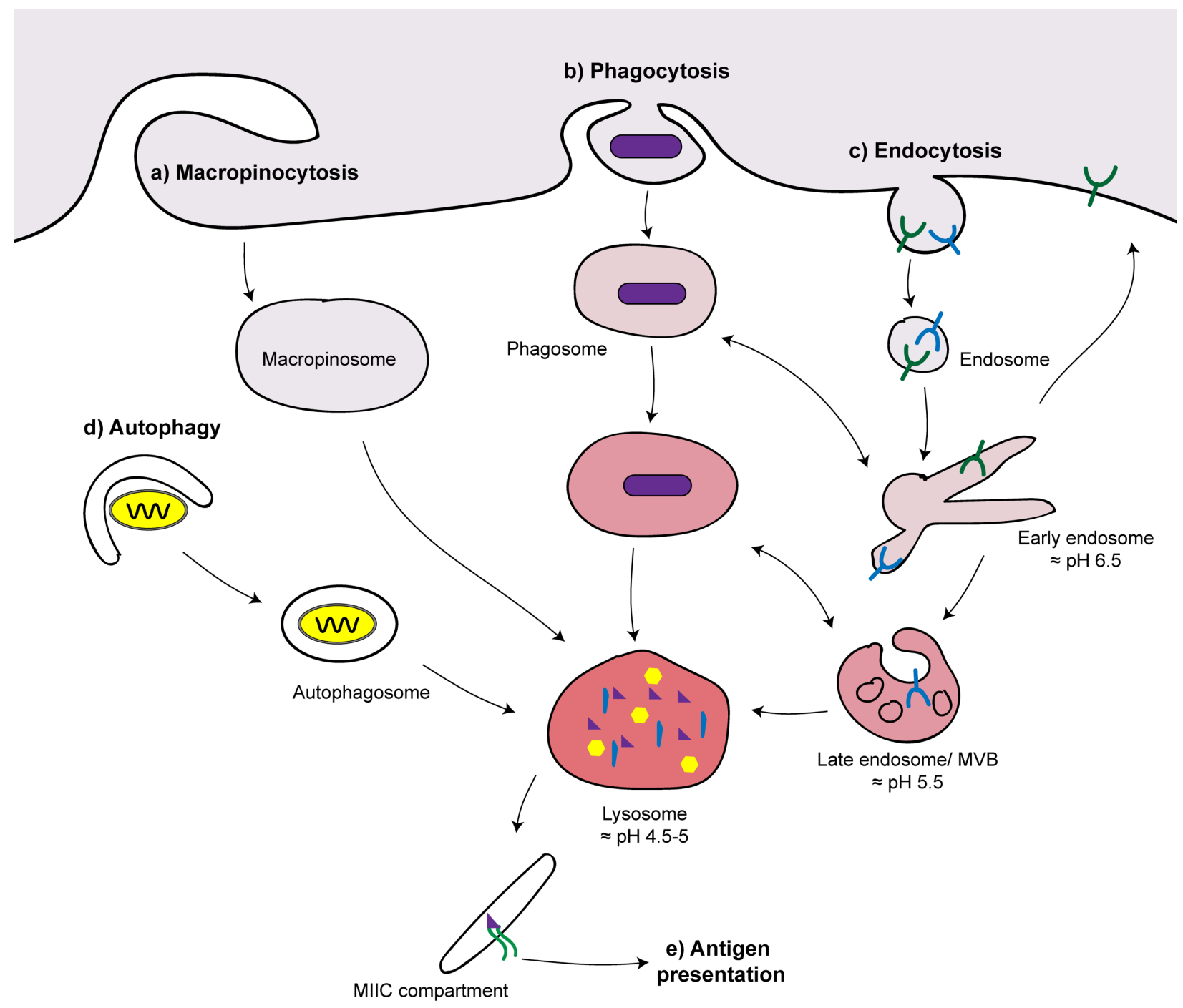


Figure 1.1: The lysosome is a convergence point for multiple membrane trafficking pathways in the cell.

(a) Macrophages and DCs perform micropinocytosis, whereby large extensions of plasma membrane (PM) ruffles collapse and fuse to non-specifically collecting extracellular material. This allows macrophages and DCs to constantly sample their extracellular environments for soluble antigens. (b) Macrophages and DCs are known as professional phagocytes, thus undergo phagocytosis. During phagocytosis, receptor bound material is internalized, via extensions of the PM called pseudopodia, into an intracellular compartment called the phagosome. Subsequently, the phagosome undergoes maturation via fusion with early and late endosomes, ultimately fusing with the lysosome forming the phagolysosome for antigen killing. (c) Endocytosis occurs in all cells, whereby small particulate material such as receptors, receptor-bound cargo and fluid-phase molecules are internalized via PM invagination into an endosome. This endosome will then fuse to the early endosome, where material can be recycled back to the PM, or trafficked to the lysosome for degradation via the late endosome. Each compartment is unique as they acquire various organelle markers and progressively acquire an acidic $\mathrm{pH}$. (d) Autophagy is an intracellular membrane trafficking pathway that occurs under various stimuli, such as when the cell is nutrient poor. Here, intracellular material is sequestered into the autophagosome and trafficked to the lysosome for degradation, and the metabolites are used as precursor molecules. (e) Antigen presentation by DCs and macrophages occurs via the formation of tubular extensions of the lysosome-related MHCII compartment (MIIC), where the antigen-MHCII complex is trafficked to the PM and presented to the adaptive immune system 
Figure 1.2

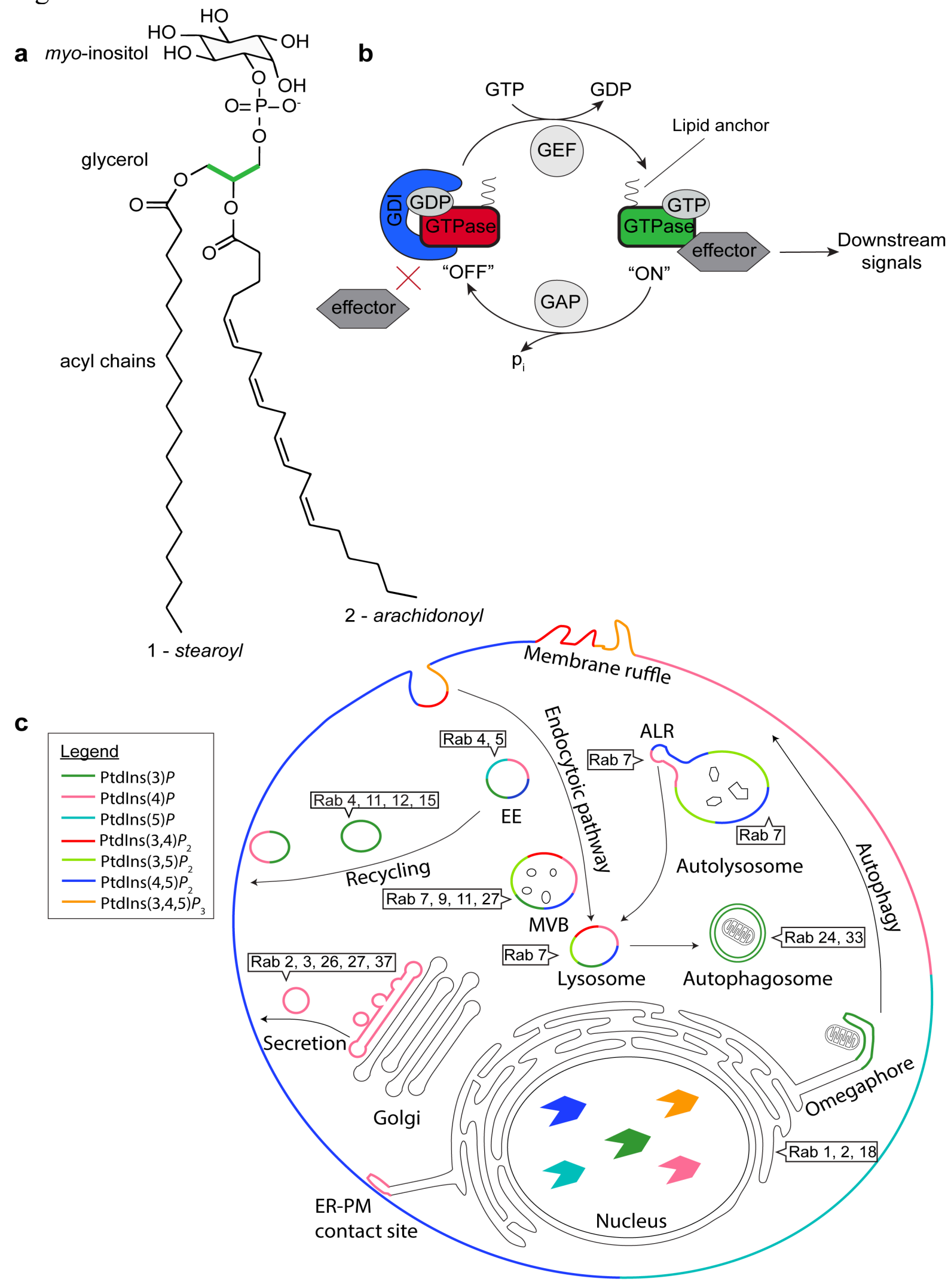


Figure 1.2: Phosphoinositides and GTPases confer organelle identity.

(a) Phosphatidylinositol is composed of three main components; a myo-inositol head group, glycerol backbone (highlighted in green) and two fatty acid tails. The fatty acids are most commonly 1-stearyol-2-aracadonyl. Phosphorylation of the myo-inositol headgroup can occur at positions 3 and/or 4 and/or 5, yielding 7 phosphorylated derivatives or PtdInsPs. (b) Small Rab GTPase activation cycle from an active GTP-bound "on" state, a conformation where they are capable of recruiting and effecting downstream molecules, to an inactive GDP-bound "off" state incapable of binding to and activating effector molecules. Cycling from GDP to GTP bound state, requires the localization of the GTPase to the target membrane through a lipid anchor which is normally masked by binding of GDI (guanine dissociation inhibitor) to the GDP-GTPase. Upon activation of GDI dissociation factor, GDI falls off, promoting the action of GEF (guanine nucleotide exchange factor), which facilitates the dissociation of GDP and quick acquisition of ubiquitous GTP, activating the GTPase. Conversely, switching to an "off" state requires the activation of the intrinsic enzymatic activity of the GTPase, which hydrolyzes the terminal phosphate group from GTP converting it to GDP and releasing an inorganic phosphate in the process. This enzymatic activity is slow and made efficient by GAP (GTPase activation protein). (c) The localization of Rab GTPase and PtdInsP species confers organelle identity and regulates basal function of a variety of organelles. Differential localization of these membrane markers allows for a diverse set of functions and regulates many signalling pathways in the cel. Please refer to (Choy, Han, \& Botelho, 2017; Zhen \& Stenmark, 2015) for reviews on localization and function of PtdInsPs and Rab GTPases, respectively. (c) was adapted from (Choy et al., 2017). 
Figure 1.3

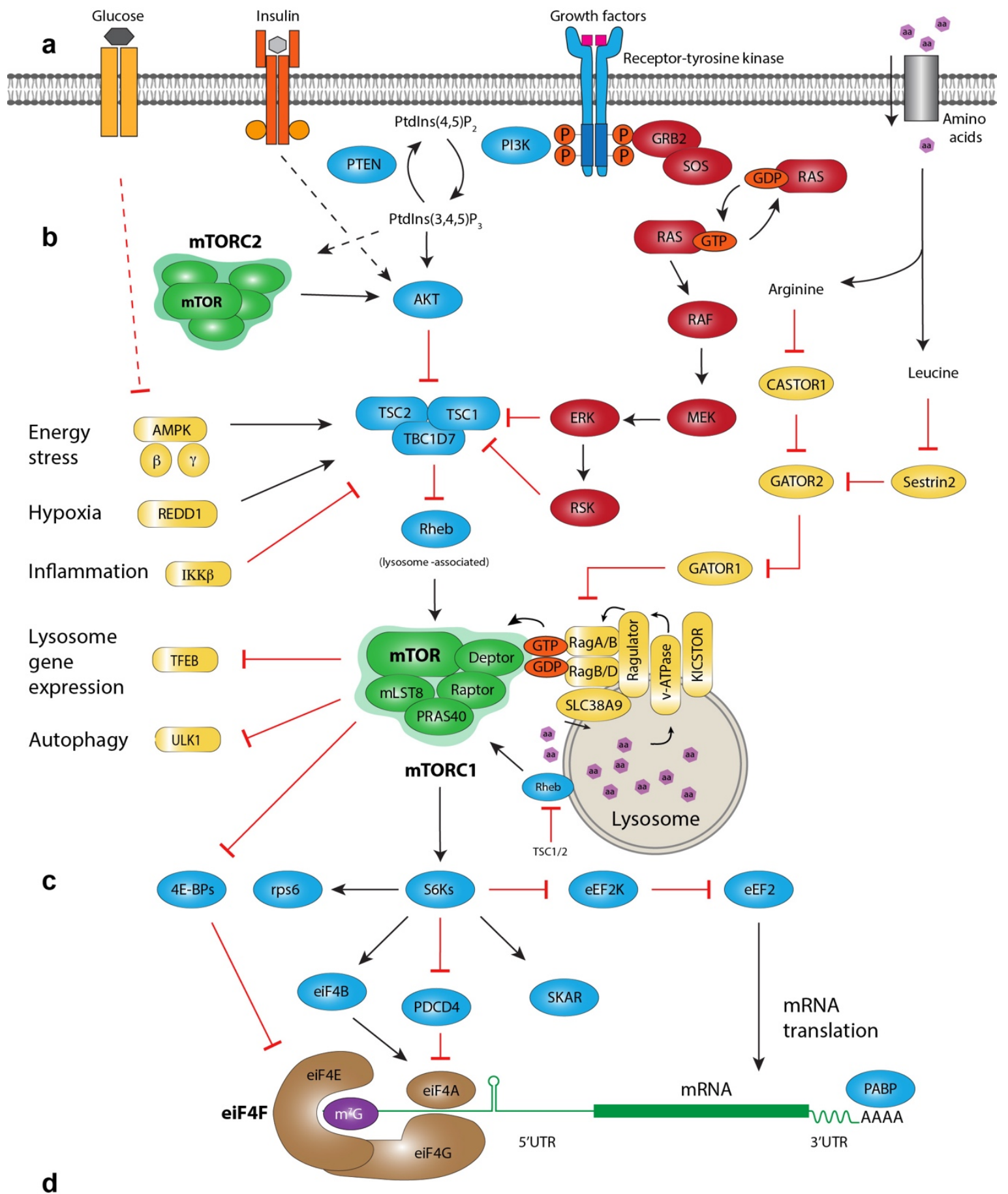

Protein synthesis

Translation initation

Translation elongation

Ribosome biogenesis

Selective translation

tRNA synthesis

\section{Anabolism}

Energy storage

Energy consumption

Lipogenesis

Mitochondria biogensis
Cell cycle

Nucleotide synthesis

G1/S phase promotion

Cell survival

\section{Catabolism}

Autophagy

Lysosome adaptation 
Figure 1.3: mTOR complex 1 activation and signalling responses.

Schematic illustrating mTORC1 activation and signalling to the mRNA translational machinery. (a) Growth factors, insulin, high glucose and amino acids all stimulate mTORC1 signalling. (b) Numerous intracellular adaptor proteins convert the extracellular signals by stimulating the PI3K/AKT and Ras/ERK signalling pathways. Additional cues promote mTORC1 activation, including inflammation and amino acids. The small Rag GTPases facilitate the translocation of mTORC1 to the surface of lysosomes for activation by Rheb GTPase, via an amino acid-dependent 'inside-out' lysosome signalling axis. Insufficient energy resources (energy stress) and hypoxia inactivate mTORC1 via the AMPK pathway and REDD1, respectively. mTORC2 also responds to agonists that stimulate the production of phosphatidylinositol-3,4,5-triphosphate $\left(\operatorname{PtdIns}(3,4,5) \mathrm{P}_{3}\right)$ and promotes the activity of AKT. (c) mTORC1 modulates mRNA translation by promoting the phosphorylation of its downstream substrates, 4E-BPs and S6Ks. S6K further promotes mRNA translation by phosphorylating a number of its own downstream targets (e.g. eIF4B, rpS6, PDCD4, and SKAR). (d) mTORC1 positively (green boxes) and negatively (red box) regulates a number of cellular signalling pathways and responses. Red inhibitory bars represent inhibitory signals, whereas black arrows indicate stimulatory signals. Solid lines indicate a direct effect on the target molecule, whereas, dotted lines indicate an indirect or not yet understood effect on the target molecule. Detailed explanations about these signalling networks and their responses are reviewed in (Inpanathan \& Botelho, 2019; Roux \& Topisirovic, 2018). Image adapted from (Roux \& Topisirovic, 2018). 
Figure 1.4

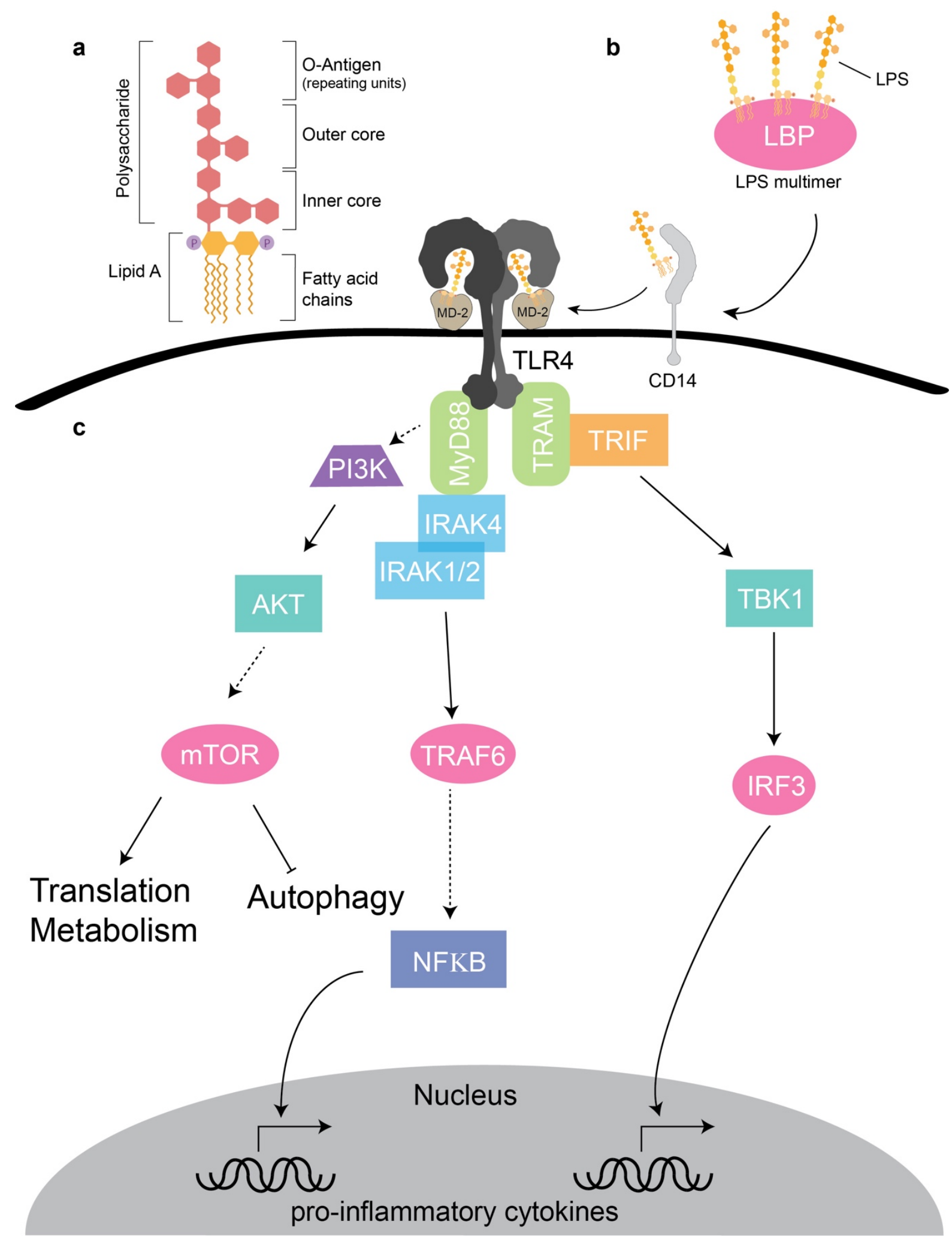


Figure 1.4: Lipopolysaccharide signals through the toll-like receptor 4 (TLR4) signalling pathway.

(a) General structure of lipopolysaccharide (LPS). LPS is composed of polysaccharide and lipid components. The lipid core can contain a range of fatty acid tails depending on strain and modification. The inner core is composed of KDO repeats that are often modified with phosphate containing groups. The outer core contains more common hexoses and is structurally more diverse than the inner core. The $\mathrm{O}$-antigen is a repeating oligosaccharide unit with one to six sugars. This is the primary structural component of LPS that differentiates bacterial strains. (b) LPS binding protein (LBP) binds to LPS in serum and is thought to help isolate LPS from the bacterial cell wall. CD14 facilitates the transfer of LPS from LBP to the TLR4/MD-2 receptor complex and modulates LPS recognition. Upon LPS recognition, TLR4 undergoes dimerization inducing a variety of intracellular signalling pathways to activate immunity. (c) LPS can trigger signalling through the MyD88-independent pathway (right) via recruitment of various adaptor proteins that lead to the activation of the transcription factor, interferon regulatory factor 3 (IRF3). Active IRF3 translocates into the nucleus and induces the production of type I interferons. Alternatively, LPS can trigger signalling through the MyD88-dependant pathway (right) via recruitment of various adaptor proteins that ultimately lead to the activation of nuclear factor kappa $\mathrm{B}(\mathrm{NF \kappa B})$ and/or mammalian target of rapamycin (mTOR). Active $\mathrm{NF \kappa B}$ translocates into the nucleus and induces the production of pro-inflammatory cytokines. Whereas, active mTOR can control autophagy progression, translation and metabolism. 
Figure 1.5

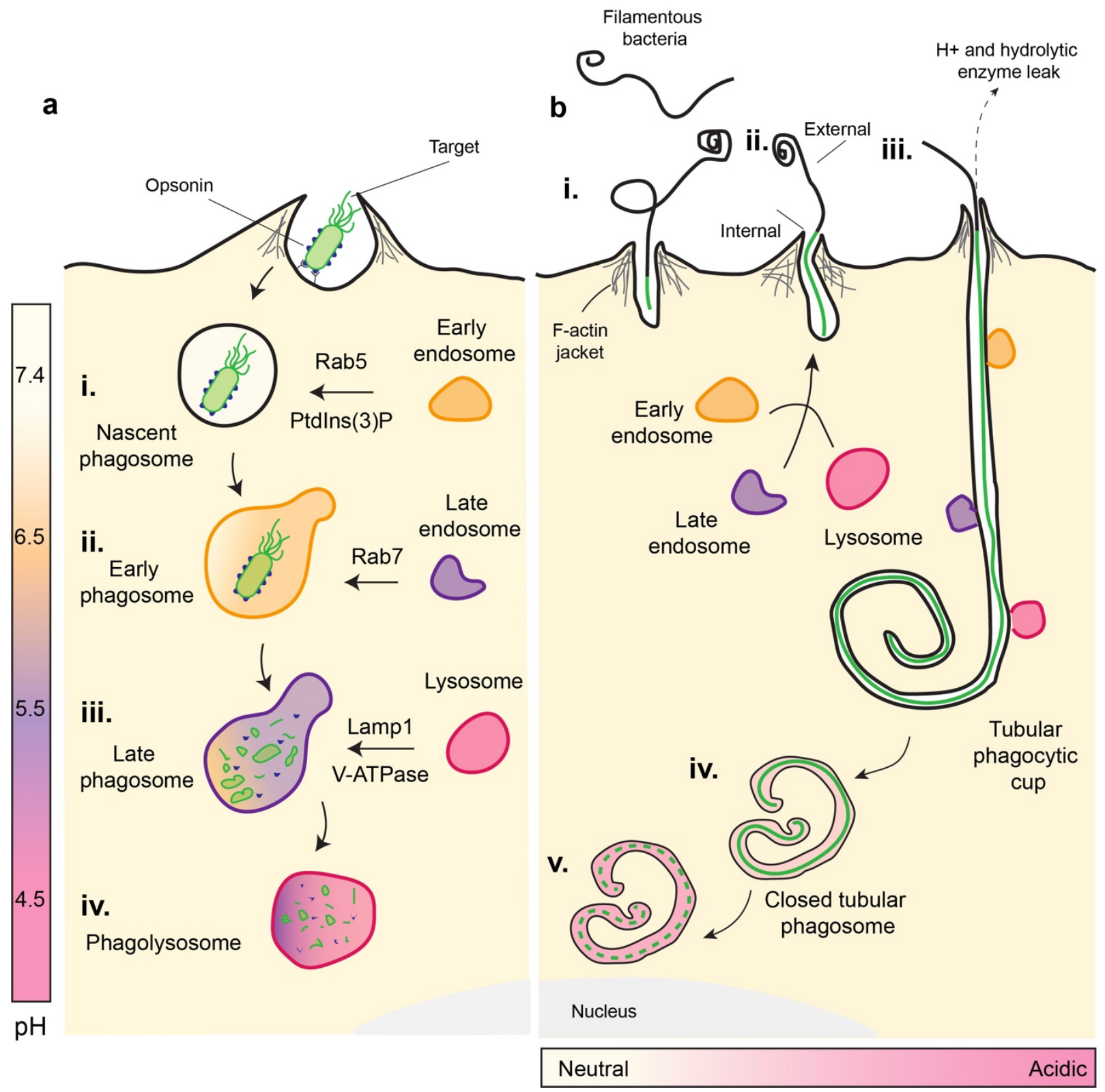


Figure 1.5: Phagosome maturation of canonical and tubular targets.

(a) Foreign particles like bacteria are recognized by the immune system and undergo phagocytosis when opsonized with antibodies like IgG. Macrophages and dendritic cells recognize the opsonin and initiate internalization. (i) Once internalized, the membrane of the nascent phagosome resembles that of the plasma membrane but loses actin and $\operatorname{PtdIns}(4,5) \mathrm{P}_{2}$, while the lumen resembles the extracellular environment. (ii) In the early phagosomal stage, phagosomes fuse with early endosomes and acquire early endosomal markers while the lumen undergoes mild acidification. (iii) Following this, early endosomal markers are lost, and late endosomal markers are acquired when fusing with late endosomes. The lumen is then further acidified. (iv) In the final stage of maturation, lysosomes fuse with phagosomes to produce a phagolyosome, where cargo degradation occurs as a result of the acidic and proteolytic environment. (b) In nature, immune cells are often challenged with targets with disparate shapes. (i) Phagocytosis of filamentous bacterial targets proceeds through a tubular phagocytic cup stage, which can last for longer than 30 minutes (Internal bacteria: green, External bacteria: black). (ii) The phagocytic cup, which is enriched in actin near the plasma membrane, undergoes maturation by fusing with endosomes and lysosomes, despite not sealing into a phagosome. (iii) The unsealed phagocytic cup and diffusion barrier established by the actin jacket, causes the leakage of protons and low molecular weight hydrolytic enzymes. As such, it is a relatively neutral structure. (iv) Eventually, the phagocytic cup seals into a tubular phagosome, where only now does the lumen acidified and become hydrolytic. (v) This antimicrobial environment allows for complete degradation of the target. 
Figure 1.6

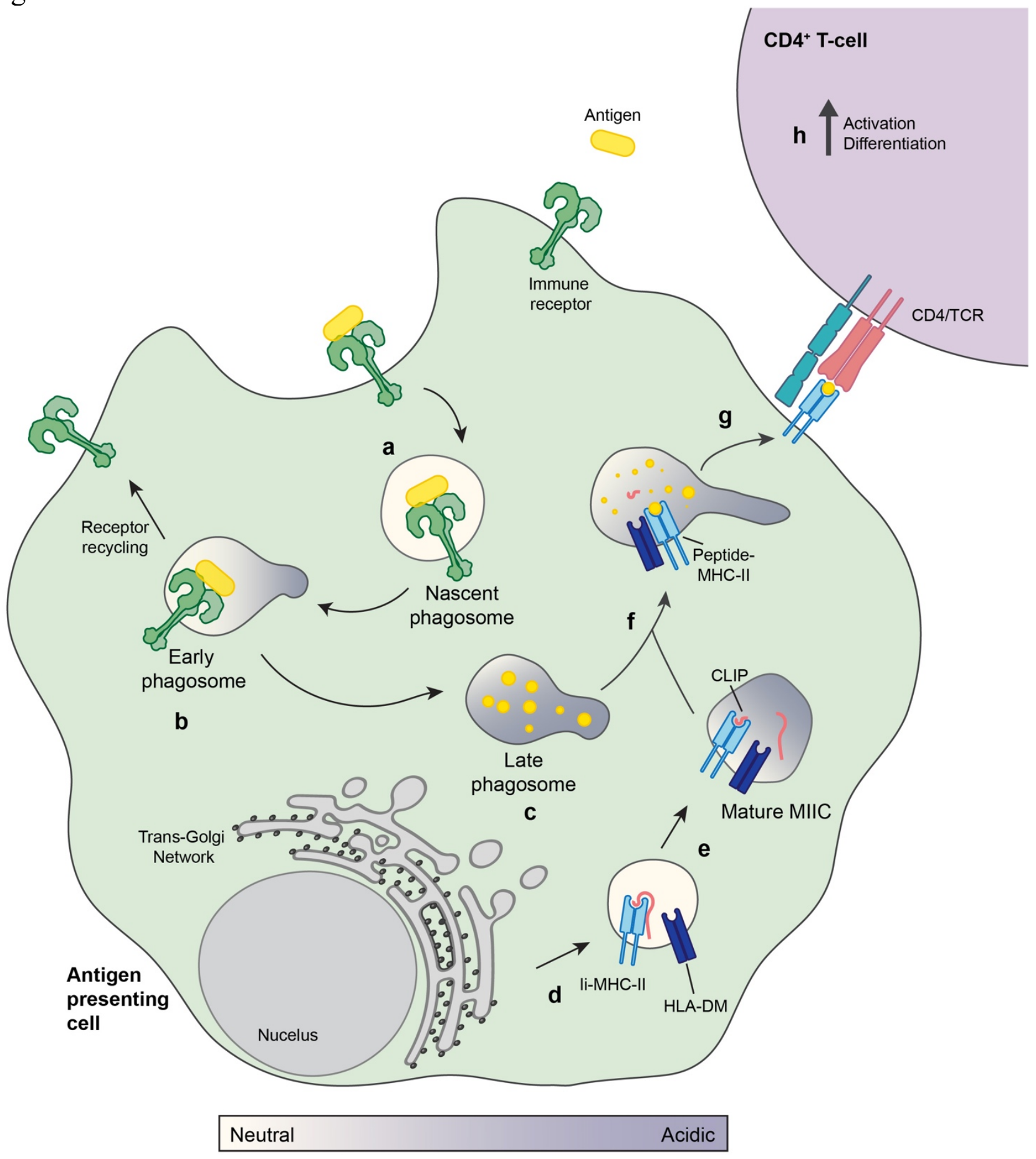


Figure 1.6: Schematic of antigen-major histocompatibility complex II presentation in dendritic cells to $\mathrm{CD} 4^{+} \mathrm{T}$-cells.

(a) Upon antigen binding to an immune receptor, antigen presenting cells like dendritic cells, internalize the particle through phagocytosis forming a nascent phagosome. (b) Within the early phagosome the immune receptor is sorted and can be recycled back to the plasma membrane. The antigen containing phagosome proceeds through the stages of maturation, progressively acquiring an acidic and degradative lumen as in (c). (d) The newly synthesized major histocompatibility complex type II (MHC-II) is trafficked from the ER-Golgi network into a lysosome-related organelle called the MHCII compartment (MIIC). To avoid premature peptide loading, the peptide binding groove is occupied by the invariant chain (Ii). (e) Upon maturation of the MIIC compartment, $\mathrm{pH}$-sensitive hydrolases cleave the invariant chain leaving the small CLIP fragment within the groove. (f) As phagosome maturation proceeds, the late phagosome fuses with the MIIC compartment. Following fusion and acquisition of a mildly acidic environment, HLA-DM catalyzes the release of the CLIP fragment from MHC-II. The empty binding groove of the nascent MHC class II molecule subsequently binds to and is loaded with an antigenic peptide. (g) The peptide antigen-MHCII complex is trafficked via tubulovesicular extensions of the MIIC compartment and is inserted into the plasma membrane for recognition by $\mathrm{CD} 4^{+} \mathrm{T}$ cells. T-cell recognition occurs through direct interaction of the antigen-MHCII complex, with the T-cell receptor (TCR) and its co-receptor, CD4. (h) Antigen presentation to T-cells is critical for T-cell priming and differentiation, translating the innate immune signal to an adaptive immune response. 


\section{Figure 1.7}

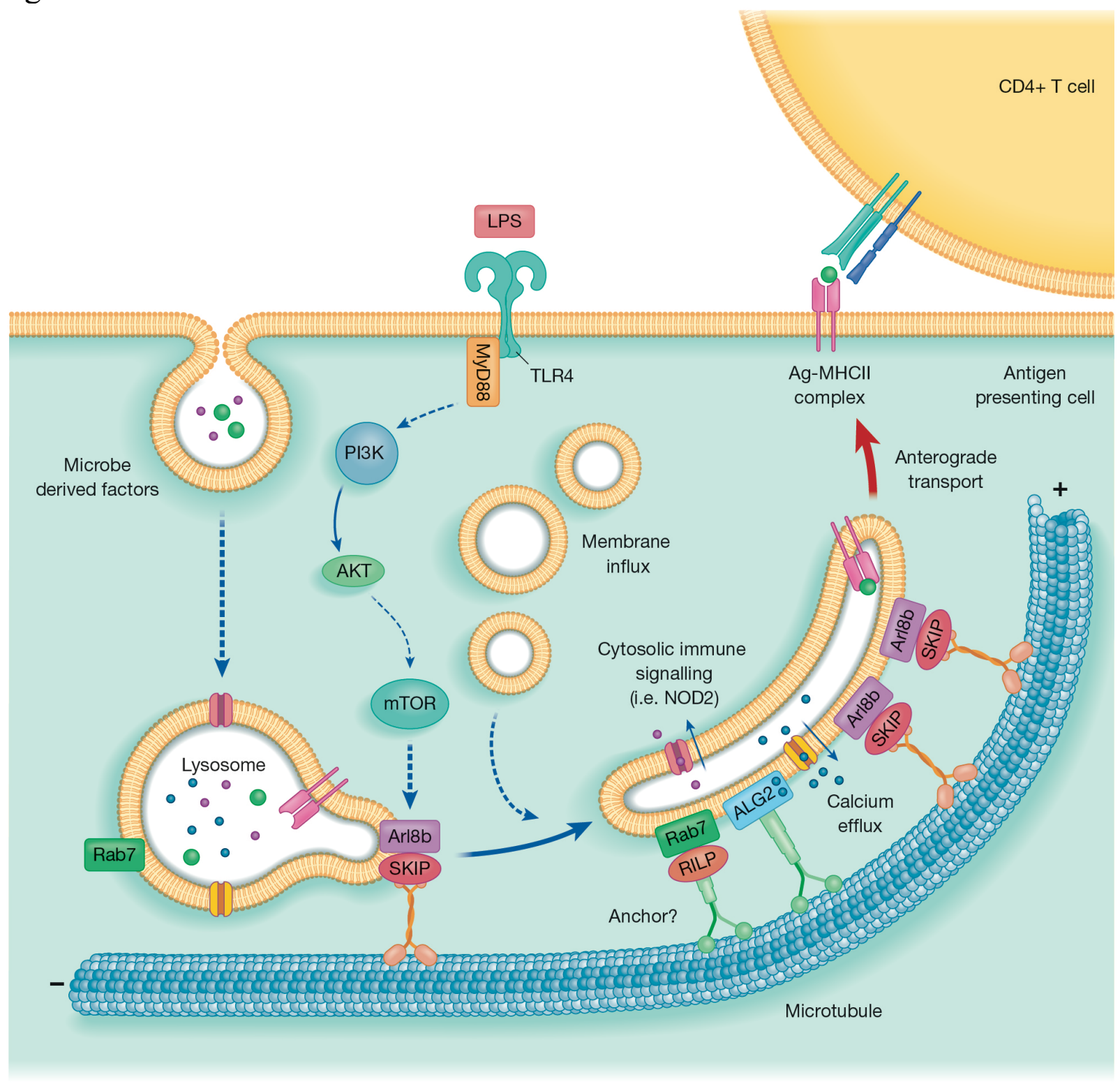

Key

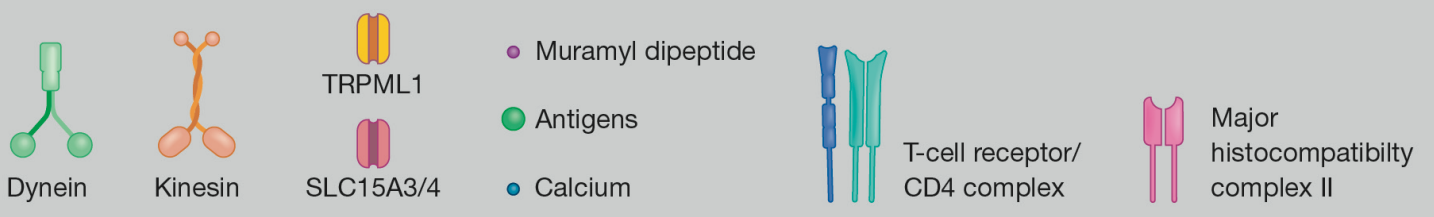


Figure 1.7: Proposed model of the known signalling pathway and mediators of lysosome tubulation.

LPS engages the PI3K-AKT-mTOR signal axis downstream of TLR4. mTOR then enhances the levels of the Arl8b GTPase on lysosomes, which likely boosts kinesin-1 activity and lysosome extension. Dynein activity is also necessary for lysosome tubulation through stimulation of the Rab7-RILP pathway and the ALG2 dynein adaptor, which is stimulated by TRPML1-mediated $\mathrm{Ca}^{2+}$ release from lysosomes. Dynein may function as a lysosomal anchor while kinesin-1 drives membrane extension towards the cell periphery, coupled with membrane influx to grow long tubules. Lysosome tubulation enhances antigen $(\mathrm{Ag})$ presentation to $\mathrm{CD}^{+} \mathrm{T}$-cells and release of microbial factors (i.e. muramyl dipeptide) into the cytosol to increase immune signalling. Solid arrows indicate established interactions or outcomes, while dashed arrows indicate predictions or extrapolated from non-immune cell systems. As described and published in (Hipolito, OspinaEscobar, \& Botelho, 2018). 
Chapter 2: Materials and methods 


\subsection{Cell Culture of cell lines and primary cells}

Murine RAW macrophage cell lines carrying CRISPR-mediated deletion of TFEB, TFE3 or both were a kind donation from Dr. Rosa Puertollano, NIH, and were previously described (Pastore et al., 2016). These cells and the wild-type RAW264.7 (TIB-71 from ATCC, Manassas, Virginia) were grown in DMEM supplemented with 5\% heat-inactivated fetal bovine serum (Wisent, St. Bruno, Canada) at $37^{\circ} \mathrm{C}$ with $5 \% \mathrm{CO} 2$. BMDCs and bone marrow-derived macrophages (BMDMs) were harvested from wild-type 7-9-week-old female C57BL/6J mice or $\mathrm{C} 3 \mathrm{H} / \mathrm{HeN}$ mice (Charles River Canada, Montreal, QC) as previously described with minor modifications (Inaba et al., 1992; Weischenfeldt \& Porse, 2008). Briefly, bone marrow was isolated from femurs and tibias through perfusion with phosphate-buffered saline (PBS) using a 27G syringe. Red blood cells were lysed using a hypoosmotic treatment. For BMDCs, cells were plated at $2 \times 10^{6} /$ well in $4 \mathrm{ml}$ of DMEM supplemented with $10 \%$ fetal bovine serum, $55 \mu \mathrm{M} \beta$-mercaptoethanol, $10 \mathrm{ng} / \mathrm{ml}$ recombinant mouse granulocyte-macrophage colony-stimulating factor (PeproTech, Rocky Hill, $\mathrm{NJ}$ ), and penicillin/streptomycin antibiotics (Wisent). Media was changed every 2 days by replacing half of the medium with fresh medium. For BMDMs, cells were plated according to experimental requirements in DMEM supplemented with $10 \%$ fetal bovine serum, $20 \mathrm{ng} / \mathrm{ml}$ recombinant mouse macrophage colony-stimulating factor (Gibco, Burlington, ON), and penicillin/streptomycin antibiotics. Media was changed every 2 days. Experiments were conducted on days 7-9. All animals were used following institutional ethics requirements.

\subsection{Lipid Transfection and plasmids}

RAW 264.7 cells were transfected with FuGENE HD (Promega Corp.) according to manufacturer's instructions and cells were used after overnight expression of constructs. The 
construct 2FYVE-GFP encodes two tandem copies of the FYVE domain of EEA1 fused to GFP (Gillooly et al., 2000) and the plasmid p40PX-GFP or mCherry encodes the PX domain of p40 Phox fused to GFP or mCherry (Kanai et al., 2001).

\subsection{Preparation of $\mathrm{pHrodo-conjugated} \mathrm{filamentous} \mathrm{bacteria}$}

PFA-killed filamentous bacteria were washed three times with $250 \mathrm{mM}$ glycine in PBS, pH 7.2 (quench buffer) and then conjugated to $\mathrm{pHrodo}^{\mathrm{TM}}$ Red succinimidyl ester (Life Technologies, Burlington, ON) as per manufacturer's direction. In brief, $10^{9}$ filamentous bacteria were resuspended in $100 \mathrm{mM}$ sodium bicarbonate buffer, $\mathrm{pH} 8.0$, and incubated with pHrodo at a final concentration of $0.5 \mathrm{mM}$. After $45 \mathrm{~min}$ at RT, the unbound pHrodo was removed by three washes in PBS. pHrodo-conjugated filamentous bacteria were finally re-suspended in PBS before use in phagocytic assays.

\section{$2.4 \quad$ Phagocytosis assays}

For the phagocytosis of filamentous bacteria, Legionella pneumophila expressing red fluorescent protein were prepared and killed with PFA as described in (Prashar et al., 2013). Filamentous bacteria were opsonized with $0.1 \mathrm{mg} / \mathrm{ml}$ anti-Legionella antibody for $1 \mathrm{~h}$ at room temperature (RT) and incubated with RAW 264.7 cells, pre-cooled at $15^{\circ} \mathrm{C}$ for $5 \mathrm{~min}$ at a ratio of 1 cell: 40 filamentous bacteria. Attachment was synchronized by spinning bacteria onto cells at $300 \mathrm{xg}$ for 5 min at $15^{\circ} \mathrm{C}$. Unattached filaments were removed by washing with PBS and the cells were then moved to a tissue culture incubator to allow phagocytosis to proceed to indicated time periods (Prashar et al., 2013).

For the phagocytosis of latex beads, $3 \mu \mathrm{m}$ poly(styrene/divinylbenzene) beads (Bangs 
Laboratories, Fishers, IN) were opsonized at a ratio of $1: 7$ with $2.86 \mathrm{mg} / \mathrm{mL}$ IgG from human serum (Sigma-Aldrich, Oakville, ON) in PBS, gyrating for 1 hour at RT. Beads were then centrifuged and washed 3 times with PBS. The final opsonized bead mixture in PBS was added at 1:10 per well. Phagocytosis synchronization was accomplished through centrifugation at 500xg for $2 \mathrm{~min}$ at $4^{\circ} \mathrm{C}$. Unbound beads were washed off with PBS and cells were then incubated in cell culture conditions.

For phagocytosis of pHrodo $^{\mathrm{TM}}$-conjugated zymosan bioparticles (Life Technologies), particles were suspended in culture medium as per manufacturer's instruction. Zymosan particles were added to cells at $0.125 \mathrm{mg} / \mathrm{mL}$ in culture medium. Phagocytosis was then synchronized through centrifugation at $500 \mathrm{xg}$ for $5 \mathrm{~min}$ at $4^{\circ} \mathrm{C}$. Following synchronization, cells were incubated for $5 \mathrm{~min}$ at $15^{\circ} \mathrm{C}$ to allow for zymosan particle coordination to receptors, unattached particles were washed off with PBS. Cells were then incubated at the defined cell culture conditions.

\section{$2.5 \quad \mathrm{pH}$ calibration of phagosomes}

Following synchronized phagocytosis, each internalized bioparticle pHrodo ${ }^{\mathrm{TM}}$ Red-conjugated filamentous bacteria or $\mathrm{pHrodo}^{\mathrm{TM}}$ Green-conjugated zymosan, underwent $\mathrm{pH}$ calibrations. For internal $\mathrm{pH}$ calibrations, cells were bathed in isotonic $\mathrm{pH}$ calibration buffers (ThermoFisher, Burlington, $\mathrm{ON})$, ranging from $\mathrm{pH} 7.5,6.5,5.5,4.5$, containing $20 \mu \mathrm{M}$ of valinomycin and 20 $\mu \mathrm{M}$ of nigericin. To ensure complete luminal homogenization at each $\mathrm{pH}$, buffers were incubated for 10 minutes prior to image acquisition using a spinning disc confocal microscope (Quorum Technologies) fitted with an Andor iXON 897 EMCCD camera. A calibration curve was generated relating the corrected mean fluorescence (background subtracted) at each $\mathrm{pH}$ to the corresponding $\mathrm{pH}$ value, following fitting the curve to a linear or one-phase exponential decay function (Figure 
3.5 \& Figure 3.8). The resulting function was used to transform initial phagosomal fluorescence measurements to a luminal $\mathrm{pH}$ value.

\subsection{Phagosome isolation}

Phagosomes containing latex beads were isolated from macrophages in 6-well tissue culture plates following 20 min of phagocytosis. Cells were incubated on ice and washed with ice-cold PBS and scraped in $1 \mathrm{~mL}$ of ice-cold homogenization buffer $[20 \mathrm{mM}$ Tris $\mathrm{pH} 7.4,1 \mathrm{mM} \mathrm{MgCl}, 1 \mathrm{mM}$ $\mathrm{CaCl}_{2}, 1 \mu \mathrm{g} / \mathrm{mL}$ RNase, $1 \mu \mathrm{g} / \mathrm{mL}$ DNase, $1 \mathrm{mM}$ AEBSF and 1:250 protease inhibitor cocktail (Sigma-Aldrich)] per well. Cells were pelleted at $500 \mathrm{xg}$ for $5 \mathrm{~min}$ at $4^{\circ} \mathrm{C}$, re-suspended with homogenization buffer, and lysed through a 22-gauge needle by syringing 10-15 times. Samples were centrifuged at $1200 \mathrm{xg}$ for $5 \mathrm{~min}$ at $4^{\circ} \mathrm{C}$, the resulting pellets were suspended in $200 \mu \mathrm{L}$ of ice-cold PBS and carefully transferred onto the sucrose gradients. Sucrose gradients were prepared freshly, prior to phagosome isolation, by centrifugation of $1 \mathrm{~mL} \mathrm{60 \%} \mathrm{sucrose} \mathrm{(in} \mathrm{PBS)} \mathrm{at} \mathrm{22,000xg}$ for $1 \mathrm{~h}$ at $4{ }^{\circ} \mathrm{C}$ and stored at $4^{\circ} \mathrm{C}$ until use. Cell lysates were applied onto sucrose gradients and centrifuged at $22,000 \mathrm{xg}$ for $15 \mathrm{~min}$ at $4^{\circ} \mathrm{C}$. Phagosomes were withdrawn from the sucrose layer and washed with ice-cold PBS. See 'SDS-PAGE and Western Blotting' for analysis of protein recruitment to isolated phagosomes.

\section{7 $\quad$ RAW 4EBP ${ }^{4 A l a}$ stable cell line production}

We generated RAW cells stably expressing the HA-4E-BP1 (4Ala) phosphorylation mutant or the corresponding empty pBABE vector as previously described (L. Rong et al., 2008), with minor modifications. Briefly, pBABE constructs were transiently transfected into the 293PhoenixAMPHO packaging cell line using Lipofectamine 2000 (ThermoFisher), as per manufacturer's 
guidelines. Following $48 \mathrm{~h}$, the viral titer was harvested and passed through a $0.45 \mu \mathrm{m}$ filter. The virus-containing medium was then used to infect RAWs in the presence of $8 \mu \mathrm{g} / \mathrm{mL}$ polybrene (Sigma-Aldrich) for $24 \mathrm{~h}$. Infection was repeated twice more. Twenty-four hours after the final infection, the medium was supplemented with $3 \mu \mathrm{g} / \mathrm{mL}$ puromycin (Sigma-Aldrich) and cells were selected for 1 week then harvested.

\subsection{Rate, retention and accumulation of pinocytic probes}

To measure pinocytosis rate or the accumulation of pinocytic cargo, BMDMs and RAW macrophages were pulsed with $1 \mathrm{mg} / \mathrm{mL}$ Lucifer yellow (ThermoFisher Scientific) for the indicated time with and without LPS from Salmonella enterica serotype minnesota Re 595 (SigmaAldrich), or after $2 \mathrm{~h}$ of LPS pre-stimulation. For pinocytic retention, BMDMs and RAW macrophages were maintained in resting conditions or stimulated with LPS for $2 \mathrm{~h}$, followed by a 30-min pulse with $1 \mathrm{mg} / \mathrm{ml}$ Lucifer yellow. Cells were then washed $3 \mathrm{x}$ with PBS, and fresh medium was added for the indicated chase periods. In all cases, cells were then washed in PBS, fixed with 4\% PFA for 15 minutes and washed in PBS. The amount of Lucifer yellow in RAW macrophages was then quantified using LSRFortessa X-20 cell flow cytometer (BD Biosciences, Mississauga, ON) in 10,000 cells per condition per experiment. Flow cytometry analysis was performed using FCS Express 5 (De Novo Software, Los Angeles, CA). For primary macrophages, Lucifer yellow-labelled cells were visualized using ImageXpress Micro Widefield High Content Screening System (Molecular Devices, Sunnyvale, CA), where $3 \times 4$ quadrants per well were acquired, and the level of probe was analysed using MetaXpress 6 (Molecular Devices). To analyze the pinocytic capacity of BMDCs following activation, cells were pre-stimulated with LPS for the indicated periods, followed by co-incubation with $50 \mu \mathrm{g} / \mathrm{mL}$ of fluorescent dextran in the 
remaining 30 min of the treatment. Cells were then washed 3x with PBS and fixed with $4 \%$ PFA for 15 minutes. Afterwards, dextran fluorescence was imaged by confocal microscopy and quantified with Volocity 6.3.0 image analysis software (PerkinElmer, Bolton, ON) by integrating intensity of dextran.

\subsection{Lysosome labeling and tubulation assays}

For lysosome labeling, cells were pulsed with $50-100 \mu \mathrm{g} / \mathrm{ml}$ Alexa 546-conjugated dextran (ThermoFisher) for $0.5-1 \mathrm{~h}$, followed by $3 \mathrm{x}$ wash with PBS and incubated with fresh medium for at least $1 \mathrm{~h}$. To induce lysosome remodelling, BMDMs and BMDCs were exposed to $100 \mathrm{ng} / \mathrm{mL}$ LPS, while RAW macrophages were incubated with $500 \mathrm{ng} / \mathrm{mL}$ for 2 hours (unless otherwise stated). For pharmacological inhibition, cells were pre-incubated for 15-20 minutes with $100 \mathrm{nM}$ torin1 (Tocris Bioscience, Minneapolis, MN), $10 \mu \mathrm{M}$ cycloheximide (Bio-Shop), $1 \mu \mathrm{M}$ LY2584702 (Selleck Chemicals, Houston, TX), $5 \mu \mathrm{M}$ Akti-1/2 (Akti) or equivalent volume of vehicle. Cells were then imaged live (unless otherwise indicated) in complete medium. Lysosomes were scored as tubules if their length was greater than $4 \mu \mathrm{m}$.

\section{$2.10 \quad$ Antigen presentation assays}

For presentation of $\mathrm{E} \alpha_{52-68}$ peptide, $\mathrm{C} 57 \mathrm{BL} / 6$ mice with $\mathrm{I}-\mathrm{A}^{\mathrm{b}}$ background were used to isolate monocytes for BMDC differentiation, and $\mathrm{C} 3 \mathrm{H} / \mathrm{HeN}$ mice with $\mathrm{I}-\mathrm{A}^{\mathrm{k}}$ background (Charles River Canada, Kingston, ON) were used for presentation of Hen-egg lysozyme (HEL). Immature BMDCs were plated on Poly-D-lysine coated glass coverslips prior to incubation with model antigens. On day 7 of differentiation, dendritic cells were incubated with $2 \mathrm{mg} / \mathrm{mL}$ of HEL (Sigma- 
Aldrich) or $60 \mu \mathrm{M}$ Ea52-68 peptide (MyBioSource, San Diego, CA) in the presence or absence of inhibitors and/or LPS, for the time points indicated.

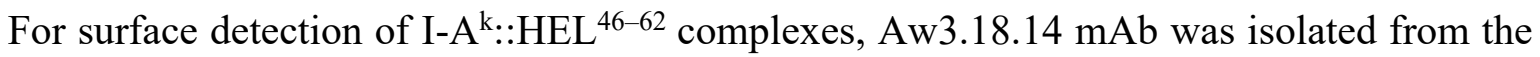
supernatant of hybridoma B-lymphocytes (ATCC, Manassas, VA). Briefly, cells were washed with ice-cold PBS 3 times, and incubated in ice-cold Aw3.18.14 for 30 minutes, then washed with PBS and fixed in 4\% PFA for 30 minutes on ice. Following, cells were incubated in Dylight-conjugated donkey polyclonal antibody against mouse (1:500; Bethyl), in standard blocking buffer for $1 \mathrm{~h}$. For presentation of I-A $:: E \alpha_{52-68}$ complexes, cells were washed 3 times with PBS and fixed in 4\% PFA for 20 mins at RT. After, cells were permeabilized in $0.1 \%$ saponin in standard blocking buffer for $1 \mathrm{~h}$. Following, cells were incubated in 1:75 mAb YAe (Santa Cruz Biotechnology, Dallas, Tx) in blocking buffer for 1 hour at RT, washed with PBS and then incubated Dylightconjugated donkey polyclonal antibodies against mouse (1:500; Bethyl), in standard blocking buffer for $1 \mathrm{~h}$. Antigen presentation of both I-A $\mathrm{A}^{\mathrm{k}:: H E L^{46-61}}$ and I-A ${ }^{\mathrm{b}:: E \alpha^{52-68}}$ complexes was visualized using confocal microscopy.

\subsection{DC: T-cell co-culture activation assays}

The I-A ${ }^{\mathrm{b}}$ restricted $\mathrm{E} \alpha$ - specific $1 \mathrm{H} 3.1 \mathrm{~T}$ cell hybridoma cell-line was used for activation assays to recognize pre-activated dendritic cells expressing $\mathrm{I}-\mathrm{A}^{\mathrm{b}}:: \mathrm{E} \alpha^{52-68}$ complexes. T-cells were cultured in RPMI-1640 medium supplemented with $10 \%$ heat-inactivated fetal bovine serum (Wisent) and $55 \mu \mathrm{M} \beta$-mercaptoethanol at $37^{\circ} \mathrm{C}$ with $5 \% \mathrm{CO}_{2}$. For activation assays, pre-activated DCs (as described in section 2.10), were mildly fixed in 1\% PFA for 15 minutes at room temperature. Following fixation cells were washed with PBS three times and then quenched thrice in complete medium for 10 minutes each, at room temperature. After, 1H3.1 T-cells and fixed- 
DCs were co-cultured at 2:1 and incubated for 40 hours at $37^{\circ} \mathrm{C}$ with $5 \% \mathrm{CO}_{2}$. Next, the tissue culture medium was collected, and T-cells were immediately isolated following centrifugation at $800 x g$ for 5 minutes. The supernatant was immediately stored in $-80^{\circ} \mathrm{C}$ for downstream IL-2 secretion analysis. To quantify T-cell activation, secreted IL-2 samples were diluted 1:10 and subsequently analyzed using Mouse IL-2 Quantikine ELISA Kit (R\&D Systems Inc, Minneapolis, $\mathrm{MN})$, as per manufacturer's specifications.

\subsection{Immunofluorescence and fluorescence microscopy}

To fix and preserve lysosome tubules in RAW cells, cells were incubated with $0.45 \%(\mathrm{v} / \mathrm{v}$ ) glutaraldehyde and $0.5 \%$ PFA (v/v) in PBS for 15 minutes at room temperature. Cells were then washed with PBS 4x, followed by incubation with $1 \mathrm{mg} / \mathrm{mL}$ ice-cold sodium borohydride (SigmaAldrich) for 5 min thrice, to abate unreacted glutaraldehyde and quench its autofluorescence.

To visualize endogenous TFEB and TFE3, cells were fixed using 4\% PFA for 15 min following treatment conditions. Cells were then treated with $100 \mathrm{mM}$ glycine in PBS to quench PFA, then in permeabilization buffer $(0.2 \%$ Triton-X, $2 \%$ BSA in PBS) for $10 \mathrm{~min}$ and then blocked for $1 \mathrm{~h}$ in 2\% BSA. Cells were incubated with rabbit anti-TFEB (1:200; Bethyl Laboratories, Montgomery, TX) or rabbit anti-TFE3 (1:500; Sigma-Aldrich) antibodies for $1 \mathrm{~h}$, followed by Dylight-conjugated donkey polyclonal antibodies against rabbit (1:500; Bethyl) for 1 h. Nuclei were counter stained with $0.4 \mu \mathrm{g} / \mathrm{mL}$ of DAPI. For LAMP1 staining, dextran-loaded cells were fixed in $0.45 \%(\mathrm{v} / \mathrm{v})$ glutaraldehyde and $0.5 \%$ PFA (v/v) in PBS for 15 minutes at room temperature. Cells were washed with PBS 3x and quenched in $25 \mathrm{mM}$ glycine for 15 mins at room temperature. Cells were permeabilized in ice-cold methanol for 3 minutes and blocked in $2 \%$ BSA for $1 \mathrm{~h}$. Cells were then incubated in primary rat anti-LAMP1 (1:100; Developmental Studies 
Hybridoma Bank) and secondary Dylight-conjugated donkey polyclonal antibodies against rat (1:500; Bethyl) for $1 \mathrm{~h}$ each. Cells were then mounted on a slide using DAKO mounting medium.

All live-cell imaging was completed at $5 \% \mathrm{CO}_{2}$ and $37{ }^{\circ} \mathrm{C}$ using environmental control chambers. Live-cell and fixed-cell imaging was done with a Quorum Diskovery spinning disc confocal microscope system equipped with a Leica DMi8 microscope connected to an Andor Zyla 4.2 Megapixel sCMOS or an iXON 897 EMCCD camera and controlled by Quorum Wave FX powered by MetaMorph software (Quorum Technologies, Guelph, ON). We also used an Olympus IX81 inverted microscope equipped with a Hamamatsu C9100-13 EMCCD camera, controlled with Volocity 6.3.0 (PerkinElmer).

For super-resolution imaging, we employed the Zeiss Elyra PS1 imaging system equipped with an Axio Observer Z1 microscope fitted with the Andor iXon3 885 detector for structure illumination microscopy (SIM) and powered by Zeiss Zen 2012 software (Zeiss Microscopy, Jena, Germany). Super-resolution image acquisition was acquired by grating for 3 rotations and 5 phases. All SIM reconstructed imaging was completed using default settings for image reconstruction; to avoid artifact formation, only images with peak/mean ratios above 20 and noise filter less then -4 were accepted. After reconstruction, Volocity 6.3.0 (PerkinElmer) image analysis software was used to measure the number of fluorescently labeled pixels. All microscopes were equipped with standard filters appropriate to the fluorophores employed in this study, optics and stage automation.

\subsection{Image and volumetric analyses}

The nuclear-to-cytosolic ratio of TFEB and TFE3 was estimated as the ratio of the mean fluorescence intensity in the nucleus over the mean intensity in the cytosol after background 
correction using ImageJ (v. 1.47 bundled with 64-bit Java). For Lamp1 and dextran colocalization, we used Mander's colocalization analysis to measure the degree of dextran colocalizing in LAMP1 structures, using the JACoP plugin in ImageJ after applying background subtraction. For volumetric analysis, we acquired confocal slices over $0.4 \mu \mathrm{m}$ z-intervals. Due to technical limitations with SIM super-resolution imaging, we sampled the area of fluorescently labeled lysosomes by acquiring 3 confocal slices in the mid-point of the cell, where we quantified the pixel area for each slice and reported an average per cell. We then used Volocity 6.3.0 image analysis software to quantify the average number of fluorescent voxels or pixels within each cell. Due to the variation in lysosomal size from experiment to experiment, we normalized the average voxel or pixel count to the corresponding control group. For lysosomal tubulation, we scored cells as positive for lysosome tubules if they displayed more than four lysosomal tubules greater than 4 $\mu \mathrm{m}$. For antigen presentation analysis, we acquired confocal slices over $0.3 \mu \mathrm{m}$ z-intervals and used Volocity to determine the total fluorescence intensity of antigen-MHCII complexes for 50100 cells per experiment. To control for background, we established a threshold fluorescence intensity measure using a no-antigen control group during each experiment. Image manipulation was done with ImageJ or Adobe Photoshop (Adobe Systems, San Jose, CA), without altering the

relative signals within images or how data may be interpreted. All figures were assembled using Adobe Illustrator (Adobe Systems).

\subsection{SDS-PAGE and Western blotting}

\subsubsection{Determination of phagosome-associated proteins and Phos-tag ${ }^{\mathrm{TM}}$ gel assays}

Proteins associated with the isolated latex beads-containing phagosomes were extracted and resolved by Western blot. To this end, phagosomes were re-suspended in Laemmli buffer 
supplemented with 1:100 protease inhibitor cocktail (Sigma-Aldrich). Proteins were then separated in a $10 \%$ SDS-PAGE, followed by protein transfer to a polyvinylidene difluoride (PVDF) membrane. Membranes were then immunoblotted using primary and secondary antibodies prepared in 5\% skim milk in Tris-buffered saline buffer with $0.1 \%$ Tween 20 (TBST) at the dilutions indicated. Proteins were detected using enhanced chemiluminescence, where protein loading was normalized to levels of human $\operatorname{IgG}$ (opsonin) in isolated phagosomes. Alternatively, we utilized Phos-tag ${ }^{\mathrm{TM}}$ gels to detect differential phosphorylation states of phagosomal markers. To this end, isolated phagosomes were re-suspended in Laemmli buffer supplemented with 1:100 protease inhibitor cocktail (Sigma-Aldrich) and PhosSTOP phosphatase inhibitor cocktail (Sigma-Alrich). SDS-PAGE gels were prepared with one fifth the strength of Phos-tag ${ }^{\mathrm{TM}}$, as recommended by the manufacturer (Wako Chemicals USA., Richmond, VA). A 5.5\% SDS-PAGE was utilized for Vps15 and Vps34, whereas UVRAG was assessed in samples run in a 7.8\% SDS-PAGE. Following the recommended electrophoresis settings, gels were agitated in $10 \mathrm{mM}$ EDTA $\mathrm{pH} 8.0$, prepared in $\mathrm{dH}_{2} \mathrm{O}$, three times for $20 \mathrm{~min}$, then rinsed three times in transfer buffer for $10 \mathrm{~min}$, after which, routine protein transfer to polyvinylidene difluoride (PVDF) membrane (EMD Millipore, Toronto, ON), immunoblotting and chemiluminescence detection conditions were used.

\subsubsection{Puromycylation assay and lysosome biogenesis markers}

For puromycylation assays, cells were treated with $10 \mu \mathrm{g} / \mathrm{mL}$ of puromycin (Sigma-Aldrich), or an equivalent water volume for the non-puromycin group, for the last 15 min of each treatment. For all western blot analysis, cells were lysed in Laemmli buffer supplemented with 1:100 protease inhibitor cocktail (Sigma-Aldrich) and PhosSTOP protease inhibitor (Roche, Mississauga, ON) 
following each treatment. Proteins were then separated in a $10 \%$ or $15 \%$ poly-acrylamide gel using standard SDS-PAGE, for high and low molecular weight proteins, respectively. Proteins were transferred to a polyvinylidene difluoride (PVDF) membrane (EMD Millipore), and blocked in 5\% skim milk or BSA, in Tris-buffered saline buffer with $0.1 \%$ Tween 20 (TBST). Membranes were then immunoblotted using the appropriate primary and secondary antibodies prepared in 5\% skim milk or BSA in TBST at the indicated dilutions. The primary antibodies used were rabbit anti-cathepsin D, ATP6V1H, ATP6V1D (GeneTex Inc., Irvine, CA), S6 ribosomal protein,

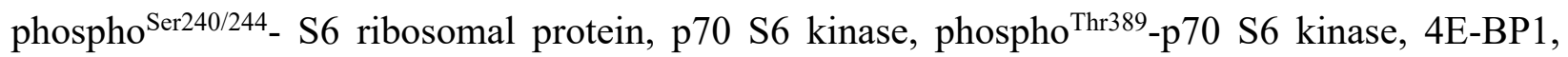

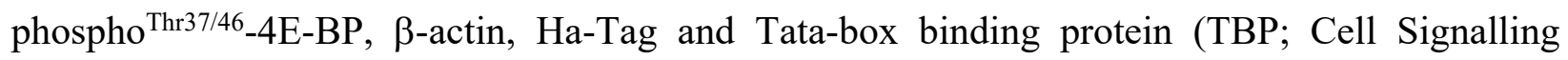
Technologies, Danvers, MA), all at 1:1,000. We also used mouse anti-puromycin clone 12D10 (1:1000, EMD Millipore), rat anti-LAMP1 and LAMP2 (abl-93) (1:200; Developmental Studies Hybridoma Bank, Iowa City, IO), rat anti-CD63 (1:1000, MBL International, Worburn, MA), rabbit anti-TRPML1 and anti-MHC Class II (I-A/I-E) (1:1000, ThermoFisher) and secondary HRP-linked antibodies raised in donkey (1:10,000, Bethyl). Proteins were detected using Clarity enhanced chemiluminescence (Bio-Rad Laboratories, Mississauga, ON) with a ChemiDoc XRS+ or ChemiDoc Touch imaging system (Bio-Rad). Protein quantification was performed using Image Lab software (Bio-Rad), where protein loading was normalized to levels of Tata box binding protein (TBP) or $\beta$-actin, and then normalized against the vehicle group.

\subsubsection{LC3 conversion autophagy assay}

We measured the conversion of LC3-I to LC3-II using immunoblotting, to measure effects on autophagy induction, in response to the pharmacological inhibitors used in our study. Primary macrophages were treated with the respective inhibitors as previously described. As a positive 
control for autophagy induction, we treated cells with Concanamycin A for 2 hours and/or cultured cells in Earle's balanced salt solution (EBSS) (Gibco) for 2 and 6 hours. Cells were lysed with Laemmli buffer (as described previously) and processed for SDS-PAGE. Proteins were separated on a $20 \%$ poly-acrylamide gel using standard SDS-PAGE. Western blotting was performed as previously described, using a primary rabbit anti-LC3 antibody (1:1000; Cell Signalling Technologies) to detect LC3-I and LC3-II abundance. For autophagy induction, immunoblots were quantified and a ratio of LC3-II to LC3-I was determined and normalized to loading control actin. Relative autophagy index was determined by comparing each treatment group to resting cells.

\subsection{Quantitative RT-PCR}

For RT-qPCR analysis in BMDMs, total RNA was extracted using the GeneJET RNA purification kit (ThermoFisher). Following RNA isolation, equal quantities of mRNA were reverse transcribed with iScript Reverse Transcription Super Mix (Bio-Rad) following manufacturer's guidelines. The subsequent cDNA was amplified for quantitative PCR using the TaqMan Fast Advanced Master Mix (ThermoFisher) with appropriate TaqMan assays. The CFX96 Touch Real-Time PCR Detection System (Bio-Rad) and CFX Manager Software (Bio-Rad) were used for amplification and analysis. The TaqMan gene expression assays (ThermoFisher) for the reference genes Abt1 (Mm00803824_m1), B2M (Mm00437762_m1) and for target genes Atp6v1h (Mm00505548_m1), Atp6v1d (Mm00445832_m1), Lamp1 (Mm00495262_m1), Mcoln1 (Mm00522550_m1), CD63 (Mm01966817_g1), Lamp2 (Mm00495267_m1) and IL-6 (Mm00446190_m1) were done in triplicate. Target gene expression was determined by relative quantification ( $\Delta \Delta \mathrm{Ct}$ method) to Abtl and the vehicle-treated control sample. 


\subsection{Polysome Profiling}

Polysome profiling was performed as detailed in Gandin et al. (Gandin et al., 2014). RAW264.7 cells were seeded in a 15-cm Petri dish and treated for $2 \mathrm{~h}$ or $6 \mathrm{~h}$ with a vehicle (DMSO), 500 $\mathrm{ng} / \mathrm{mL}$ LPS from Salmonella enterica serotype minnesota Re 595, $100 \mathrm{nM}$ torin 1 for $2 \mathrm{~h}$ only, or the combination of LPS $(500 \mathrm{ng} / \mathrm{mL})$ and torin $1(100 \mathrm{nM})$ whereby cells were pre-treated for 15 minutes with torin 1 before stimulation with LPS. Cells were harvested at $80 \%$ confluency, washed twice with ice-cold PBS containing $100 \mu \mathrm{g} / \mathrm{mL}$ cycloheximide and then lysed in hypotonic lysis buffer (5 mM Tris $\mathrm{HCl}, \mathrm{pH}$ 7.5, $2.5 \mathrm{mM} \mathrm{MgCl}, 1.5 \mathrm{mM} \mathrm{KCl}, 100 \mu \mathrm{g} / \mathrm{ml}$ cycloheximide, $2 \mathrm{mM}$ dithiothreitol (DTT), $0.5 \%$ Triton, and $0.5 \%$ sodium deoxycholate). Optical density values at 260 $\mathrm{nm}\left(\mathrm{OD}_{260}\right)$ were measured in each lysate and $15 \mathrm{OD}_{260}$ were then loaded on $5-50 \%$ sucrose gradients generated using Gradient Master (Biocomp, Fredericton, New Brunswick). Ten percent of lysates were saved as input samples for total RNA extraction. Sucrose gradients were subjected to ultracentrifugation (SW41 Ti 11E1698 rotor; Beckman at 260,000xg for $2 \mathrm{~h}$ at $4{ }^{\circ} \mathrm{C}$ ) and fractionated by displacement using $60 \%$ sucrose $/ 0.01 \%$ bromophenol blue on an ISCO Foxy fraction collector ( $35 \mathrm{~s}$ for each fraction, or $\sim 750 \mu \mathrm{L}$ per fraction) equipped with an ultraviolet lamp for continuous absorbance monitoring at $254 \mathrm{~nm}$. Fractions were flash-frozen immediately after fractionation and stored at $-80^{\circ} \mathrm{C}$. RNA was isolated with Trizol (Thermofisher) as per manufacturer's instruction. All experiments were carried out at least three independent biological replicates $(\mathrm{n}=3)$. Samples for polysome profiling were collected by Kristofferson Tandoc from the Topirisovic lab at McGill University.

Reverse transcription and RT-qPCR were performed with iScript Reverse Transcription Super Mix (Bio-Rad) and TaqMan Fast Advanced Master Mix (ThermoFisher), respectively. All experiments were carried out at least three independent biological replicates $(n=3)$. Analyses were 
carried out using relative standard curve method as instructed by the manufacturer. The following TaqMan assays were completed using the primers described above for quantitative RT-PCR and in addition to Actb (Mm0261958__g1) and Ppia (Mm02342430_g1).

\subsection{Phosphoinositide labeling and HPLC-coupled flow scintillation}

Cells were incubated in tritium labeling medium [inositol-free DMEM (MP Biomedical) supplemented with $10 \mu \mathrm{Ci} / \mathrm{mL}$ myo-[2- $\left.{ }^{3} \mathrm{H}(\mathrm{N})\right]$ inositol (Perkin Elmer, Woodbridge, ON), $4 \mathrm{mM}$ L-glutamine (Sigma-Aldrich), insulin-transferrin-selenium-ethanolamine (Gibco), 10\% dialyzed FBS (Gibco), $20 \mathrm{mM}$ Hepes (Gibco), and Penicillin-Streptomycin mix (Sigma-Aldrich)] for $24 \mathrm{~h}$. After incubation, tritium labeling medium was removed and replaced with cell culture medium to perform experimental conditions for $20 \mathrm{~min}$. Cells were lysed on ice with $4.5 \%$ perchloric acid $(\mathrm{v} / \mathrm{v})$ for $15 \mathrm{~min}$ and pelleted at $12,000 \mathrm{xg}$ for $10 \mathrm{~min}$. Pellets were washed with $0.1 \mathrm{M} \mathrm{EDTA} \mathrm{pH}$ 8.0 , and sonicated in $50 \mu \mathrm{L}$ of water. Samples were incubated in phospholipid deacylation reagent [45.7\% methanol, 10.7\% methylamine, $11.4 \% 1$-butanol $(\mathrm{v} / \mathrm{v})]$ for 50 minutes at $53^{\circ} \mathrm{C}$. Samples were subsequently dried in speed-vacuum and re-suspended in water by sonication twice before a final drying step for storing them. To resume analysis, dried samples were re-suspended in a 1.5:1 ratio of water to extraction reagent [1-butanol/ethyl ether/ethyl formate (20:4:1)], vortexed for 5 min and centrifuged for $2 \mathrm{~min}$. The aqueous layer was carefully isolated and extracted twice more, then vacuum dried and re-suspended in $50 \mu \mathrm{L}$ of water. For each sample, equal counts of tritium were separated by HPLC (Agilent Technologies) through an anion exchange 4.6 x 250-mm column (Phenomenex) and subjected to a gradient of water and $1 \mathrm{M}\left(\mathrm{NH}_{4}\right)_{2} \mathrm{HPO}_{4} \mathrm{pH}$ 3.8. For HPLC separation protocol see (Ho, Choy, \& Botelho, 2016). The radiolabeled eluate was detected with a 1:2 ratio of eluate to scintillation fluid by $\beta$-RAM 4 (LabLogic) and analyzed by Laura 4 software. PtdIns(3)P and PtdIns(4,5) $\mathrm{P}_{2}$ levels were normalized against the parent PtdIns peak. 


\subsection{Statistical Analysis}

Data shown are mean \pm SEM or SD from at least three independent experiments, unless otherwise stated. Using GraphPad Prism software (GraphPad Software, Inc.), statistical analysis was performed using two-tailed, Student's $t$ test, analysis of variance (ANOVA) or analysis of covariance (ANCOVA). Appropriate post-hoc tests following ANOVA or ANCOVA were also performed to determine particular differences between group means. A 95\% confidence interval was used to determine statistical significance and $p \leq 0.05$ was considered to be statistically significant. 
Chapter 3: Maturation of tubular phagosomes 


\subsection{Chapter rationale and overview}

Immune cells have adapted a variety of mechanisms that enhance their degradative capacities. The process of phagocytosis allows phagocytes to internalize an invading particle to process and degrade it internally. The stages of phagocytosis have largely been elucidated through studies using canonical spheroidal phagosomes. A significant amount of research has identified the necessary stages of sequential organelle fusion, transient acquisition of molecular traits characteristic to each stage, which ultimately leads to the transformation of a nascent inert phagosome into a phagolysosome where it is digested. What are less understood however, are the mechanisms that coordinate the timing of the association and dissociation of the markers.

In nature phagocytes are challenged with a variety of targets with different shapes and sizes. Targets of filamentous morphology can present a hurdle for phagocytosis. In fact, some bacterial species adopt a filamentous morphology to evade phagocytosis (Justice et al., 2008; Yang, Blair, \& Salama, 2016) and macrophages fail to efficiently internalize filamentous targets when they are engaged by their long axis (Champion, Walker, \& Mitragotri, 2008). Nevertheless, phagocytosis of filamentous bacteria advances successfully when macrophages capture and engulf filaments by one of their ends (Möller et al., 2012). Using filamentous L. pneumophila Prashar et al (2013) discovered that upon internalization, a tubular phagocytic cup forms and its closure only occurs once the whole target is enclosed, which often surpasses the time needed for uptake of model spheroidal targets. Interestingly, they found that the pericytoplasmic portions of the tPC sequentially fuse with endosomes and lysosomes prior to sealing, and recommend it as a suitable model to study phagosome maturation.

In this chapter, I investigated the role that $\mathrm{pH}$ plays in the turnover of the early endosomal marker PtdIns(3)P, during phagosome maturation using both tubular phagosomes and canonical 
spherical targets. Understanding the intricate steps of this process can help us better understand how pathogens may co-opt phagocytes to evade phagolysosome degradation and clearance from the immune system. We first set out to investigate the temporal and spatial recruitment of early and late phagosomal markers on the tubular phagocytic cup through overexpression of markers and fluorescence microscopy. Due to the presence of a possible $\mathrm{H}^{+}$gradient established by the actin-jacket diffusion barrier; I investigated the role of $\mathrm{pH}$ in the recruitment of early endosomal markers. A combination of biochemical, fluorescence microscopy and radiolabeling techniques were used. Ultimately, I identified a novel pH-based mechanism which modulates the association of Vps34 complex II with endomembranes controlling PtdIns(3)P dynamics.

This work was completed in collaboration with the Terebiznik lab at University of Toronto Scarborough resulting in a first co-authorship manuscript published in the Journal of Cell Biology (Naufer, Hipolito et al., 2018). As a result, I present the work described in the cited article in totality to maintain a flow of the story and narrative. Unless otherwise indicated in the captions of the presented figures, all data presented was produced, analyzed and put together by me. 


\subsection{Results}

\subsubsection{Vps34 controls PtdIns(3)P persistence on tPCs during maturation}

During canonical phagocytosis, the phagosome acquires the early endosomal markers Rab5 and localized synthesis of PtdIns(3)P, by the turnover of PtdInsP to the monophosphorylated form by Class III PI3K Vps34. Localization of these markers allows for the recruitment of early endosomal marker 1 (EEA1), necessary for subsequent tethering and fusion with later compartments. We first investigated the localization of the early and late markers on the growing tPC.

To test this, we used PFA-killed filamentous L. pneumophila (f-LP) to avoid the effect of its secreted toxins on phagocytosis. Following 5 minutes of phagocytosis and the onset of a small tPC, both Rab5 and EEA1 were recruited but the region was devoid of LAMP1 (Figure 3.1a, c). By 30 minutes of internalization, the entire tPC lacked both Rab5 and EEA1, and was decorated with LAMP1 (Figure 3.1b, c), mimicking the canonical phagosome whereby earlier markers precede the acquisition of late markers in a temporally-regulated process. Next we investigated the dynamics of PtdIns(3)P on the growing tPC using 2FYVE-GFP and p40PX-GFP probes (Ellson et al., 2001; Gillooly et al., 2000; Kanai et al., 2001; Vieira et al., 2001). Interestingly, both probes stayed on tPCs after 45 minutes of phagocytosis (Figure 3.1d, e). Remarkably, even after 5 minutes, both probes co-localized with late endosomal markers LAMP1, Rab7 and RILP-c33 (a probe for active GTP-Rab7) on distal portions of the tPCs (Figure 3.1f, g). Both 2FYVE-GFP and p40PX-GFP probes were only stripped from tPCs after phagosome closure (Figure 3.1f - right panel), as is characteristic with canonical phagosomes. Thus far our results indicate that PtdIns(3)P co-localizes with LAMP1-positive tPCs following loss of early markers, providing a unique temporal and spatially resolved tool to study the signals that may coordinate PtdIns(3)P turnover during phagosome maturation. 
Next, we sought to investigate the source of PtdIns(3)P maintenance on tPCs. Increased lipid biosynthesis or decreased lipid turnover could increase levels of PtdIns(3)P. We investigated the role of the different classes of PI3Ks, first we blocked all classes with a pan-PtdIns 3-kinase inhibitor, LY294002 (Gharbi et al., 2007) and then used ZSTK474, a Class I PI3K inhibitor (Kong \& Yamori, 2007). Treatment with LY294002 abrogated all PtdIns(3)P production on tPCs, whereas, ZSTK474 did not affect 2FYVE-GFP recruitment (Figure A1a, b).

Subsequently, we investigated the role of the Class III PI3K, Vps34, using the established Vps34-IN1 inhibitor (Bago et al., 2014). Treatment of RAW cells with Vps34-IN1 during phagocytosis of f-LP completely blocked 2FYVE-GFP recruitment onto tPCs, indicating Ptdns(3)P synthesis is likely being regulated by Vps34. To dissect if Ptdns(3)P present on tPCs undergoes turnover, we allowed phagocytosis to proceed and accumulation of 2FYVE-GFP for 20 minutes on tPCs before treatment with Vps34-IN1. Twenty minutes post treatment, cells were fixed and imaged. Despite the delayed addition of Vps34-IN, 2FYVE-GFP significantly reduced onto tPCs, demonstrating that PtdIns(3)P was undergoing turnover during tPC biogenesis (Figure 3.2a, b, c). To examine PtdIns(3)P turnover, we investigated the role of PIKfyve, which phosphorylates PtdIns(3)P to produce PtdIns(3,5) $\mathrm{P}_{2}$ (Ho, Alghamdi, \& Botelho, 2012; McCartney, Zhang, \& Weisman, 2014). Treatment of RAW cells with apilimod, a specific inhibitor of PIKfyve (Cai et al., 2013), strongly increased the accumulation of 2FYVE-GFP onto tPCs (Figure 3.2d). Together, these data suggest that PtdIns(3)P maintenance onto tPCs is due to increased synthesis by Vps34 and not due to a defect in turnover. Therefore, this suggests that the role of PtdIns(3)P in $\mathrm{PC}$ phagocytosis is similar to canonical phagocytosis, but it appears to be temporally regulated. 


\subsubsection{PtdIns(3)P loss at distal tips of tPCs correlates with local acidification}

After, we differentiated the temporal fate of PtdIns(3)P on growing tPCs using live-cell time-lapse microscopy. As depicted in the time-lapse micrographs in (Figure 3.3a), 2FYVE-GFP was present all along the growing tPC within 3:00 (mm:ss), whereby, once a threshold was met following 3:15 (mm:ss) and thereafter, 2FYVE-GFP began to disappear from the most distal end, while staying at the most promixal portion. Interestingly, we determined a length threshold of $20 \mu \mathrm{m}$ (Figure 3.3b) using fixed cell imaging and correlating the total length of the tPC to its length that was positive for 2FYVE-GFP or p40PX-GFP. As shown in Figure A1c-d during early phagocytosis, the tPC length tends to be decorated with the PtdIns(3)P probes, however, the strong correlation between these parameters is lost as GFP-fluorescence began to disappear from the most internalized portion of tPCs, usually longer than $20 \mu \mathrm{m}$ (Figure A1c-d \& Figure 3.3b). These data suggest that the dynamics of PtdIns(3)P is regulated by differential signalling depending on the length of tPC.

Prashar et al. (2013), previously hypothesized that the actin jacket formed near the proximal portion of the growing tPC may act as a diffusion barrier for the transfer of luminal products of the tPC, such as $\mathrm{H}^{+}$, as they reported that tPCs do acquire V-ATPases but do not acidify until phagosome closure. Since acidification is considered a mark of PtdIns(3)P-negative phagolysosomes, we wanted to investigate if acidification of the tPC may affect PtdIns(3)P dynamics. To this end, we conjugated the fluorescent $\mathrm{pH}$-sensor, pHRodo, onto f-LP (as described in methods) and employed live-cell imaging of a macrophage internalizing the $\mathrm{pH}$-sensitive $\mathrm{f}$-LP to determine if a $\mathrm{pH}$ gradient was present. As illustrated in Figure A2, acidification of tPCs, depicted by the increase in pHRodo intensity, occurred at the most distal portion of the internalized filament and corresponded with the loss of PtdIns(3)P as shown by the decrease in p40PX-GFP. 
Whereas, the most proximal portion contained augmented levels of p40PX-GFP and decreased pHRodo intensity. These data suggest that it may be possible that tPC acidification may be a result of a balance between $\mathrm{H}^{+}$leakage across the actin-jacket diffusion barrier and the activity of $\mathrm{V}$ ATPase pumps.

\subsubsection{Acidification of tPCs correlates with the loss of $\operatorname{PtdIns}(3) \mathrm{P}$}

To confirm this, we investigated the dynamics of PtdIns(3)P and changes in pHRodo intensity, by forcing luminal acidification and neutralization of the tPC. Due to the presence of the actin-jacket diffusion barrier, we suspect that we can force luminal acidification of the tPC in macrophages that are engaged in early phagocytosis of a f-LP particle. Using live-cell time-lapse microscopy, we bathed macrophages already engaged in early phagocytosis of a pHRodo-conjugated f-Lp in media adjusted to a $\mathrm{pH}$ of 4.5 and observed the fate of p40PX-GFP over time (Figure 3.4a). First, we observed that upon internalization of a $\mathrm{pH}$-sensitive f-Lp, pHRodo fluorescence intensity increased over time, indicating a successful decrease in $\mathrm{pH}$ of the luminal tPC. We also tracked the fate of proximally localized PtdIns(3)P on the tPC upon forced acidification. The rise in luminal acidity corresponded to the disappearance of p40PX-GFP from the membrane. Next, we quantified the proportion of internal tPCs positive for PtdIns(3)P in resting macrophages and those bathed in the acidic media and observed a significant decrease in p40PX-GFP as early as 10 minutes following media addition (Figure 3.4b). These data suggest that luminal $\mathrm{pH}$ of the tPC affects the fate of PtdIns(3)P.

Afterwards, we investigated if V-ATPase function may dictate PtdIns(3)P dynamics. We treated cells with the proton pump inhibitor concanamycin A (ConA) to hinder acidification and tracked p40PX-GFP on internalized fragments. Treatment with ConA stabilized PtdIns(3)P in the 
most distal regions of tPCs (Figure 3.4c), as filamentous phagosomes of similar length in ConAtreated cells were positive for p40PX-GFP at the distal region compared to control. Additionally, filamentous phagosomes of similar lengths in ConA-treated cells, were still positive for p40PXGFP following closure, compared to the control group (Figure 3.4d). Therefore indicating that ConA treatment abated turnover of PtdIns(3)P.

\subsubsection{Luminal $\mathrm{pH}$ of 6.2 controls PtdIns(3)P turnover on tPCs}

Thus far, our data suggest that luminal acidification of tPCs controls PtdIns(3)P dynamics. We were interested in determining the $\mathrm{pH}$ at which the lipid was eliminated on tPCs. We used an internal $\mathrm{pH}$-calibration system on live-macrophages containing bacteria of $10-15 \mu \mathrm{m}$ in length. This defined length allows for uniform distribution and dissociation of PtdIns(3)P markers from the tPC membrane. Following synchronized phagocytosis and internalization of pHRodoconjugated f-Lp, we acquired confocal fluorescent images of cells before and after internal $\mathrm{pH}$ calibration. For $\mathrm{pH}$ calibration, we bathed cells for 10 minutes in each isotonic $\mathrm{pH}$ calibration buffer, as described in the methods section, and generated a calibration curve for each filament (Figure 3.5a). To determine the $\mathrm{pH}$ at which PtdIns(3)P falls from membranes, we clustered pHRodo f-Lp's positive and negative for $\mathrm{p} 40 \mathrm{PX}-\mathrm{GFP}$ and measure its resting $\mathrm{pH}$ through $\mathrm{pHR}$ odo calibration. Interestingly, we determined that p40PX-GFP-positive phagosomes had a mean $\mathrm{pH}$ of 6.6, while p40PX-GFP-divested phagosomes had a $\mathrm{pH}$ mean of 5.7. Comparing the range of quantified phagosomes from three independent experiments, we estimate that the phagosomes devoid of PtdIns(3)P tend have a pH of 6.2 (Figure 3.5c).

In order to ensure lipid loss was not due to the effect of $\mathrm{pH}$ on p40PX-GFP or 2FYVEGFP binding to the lipid, our collaborators, Suriakarthiga Ganesan and Vanina Zaremberg, from 
the University of Calgary, used lipid overlay assays to determine the effect of $\mathrm{pH}$ on probe binding (Figure A3a-c). They discovered that both probes bound to PtdIns(3)P more effectively in media set to pHs less then 6.5 (Figure A3d). These data is consistent with (He et al., 2009) and (Lee et al., 2005). In addition, p40PX-GFP remained associated with enclosed phagosomes in apilimodtreated cells despite significant acidification (Figure 3.5c). Together, these data show a causality between $\mathrm{pH}$ and the fate of PtdIns(3)P on tPCs. 


\subsection{3 pH controls the lifespan of PtdIns(3)P on canonical phagosomes}

Using the spatiotemporal resolution achieved along the tubular phagocytic cup, we were able to identify a novel $\mathrm{pH}$-based mechanism that controls PtdIns(3)P dynamics during maturation. We next were interested in investigating if the same phenomenon occurs during canonical phagocytosis of spheroidal targets such as phagosomes containing latex beads.

We first fed IgG-opsonized latex beads to macrophages and investigated the dynamics of PtdIns(3)P on phagosomes over time. As expected, we saw a decrease in 2FYVE-GFP recruitment in a time-dependent manner (Figure 3.6). We then used $\mathrm{ConA}$ and $\mathrm{NH}_{4} \mathrm{Cl}$ to neutralize the luminal environment of phagosomes and lysosomes, impeding phagosome acidification. Upon neutralization, 2FYVE-GFP levels were significantly augmented on phagosomal membranes overtime. These results support a role for a $\mathrm{pH}$-dependent mechanism controlling PtdIns(3)P dynamics.

To complement this experiment, we were interested in following PtdIns(3)P dynamics on another biologically relevant phagocytic pathway that does not engage the Fc $\gamma$ receptor pathway. We employed pHRodo-conjugated zymosan bioparticles, which engage an array immune receptors such as Dectin-1 and TLR2, and G-protein signalling upon phagocytosis (Gantner, Simmons, Canavera, Akira, \& Underhill, 2003; Huang et al., 2014; Ozinsky et al., 2000). To this end, we followed the dynamics of $\mathrm{p} 40 \mathrm{PX}-\mathrm{mCh}$ recruitment and pHRodo-zymosan intensity over time (Figure 3.7a). As expected, there was an inverse relationship between PtdIns(3)P recruitment and pHRodo intensity in the control group, where phagosomal pHRodo fluorescence increased over 10 minutes, upon progressive depletion of $\mathrm{p} 40 \mathrm{PX}-\mathrm{mCh}$ (Figure 3.7b). Similar to our experiments with IgG-opsonized latex beads, when we blocked phagosome acidification using ConA, p40PX-

$\mathrm{mCh}$ recruitment drastically persisted over time (Figure 3.7b). Although $\mathrm{p} 40 \mathrm{PX}-\mathrm{mCh}$ did 
eventually fall off of the phagosomal membrane, indicating there may be additional mechanisms which signal PtdIns(3)P depletion.

In contrast, when we forced acidification in the ConA-treated group by bathing cells in

media set to $\mathrm{pH} 4$, we forced the dissociation of $\mathrm{p} 40 \mathrm{PX}-\mathrm{mCh}$ from phagosomal membranes (Figure 3.7b). Although one may argue that an acidic $\mathrm{pH}$ may quench fluorophore fluorescence as is the case with GFP (Haupts, Maiti, Schwille, \& Webb, 1998), the mCherry fluorophore has been shown to be stable under acidic conditions (Doherty, Bailey, \& Lewis, 2010; Stoddard \& Rolland, 2019). Additionally, mCherry fluorescence did not appear to be affected, as observed by the strong cytosolic fluorescence still evident following forced acidification. When we take into account that ConA-alone prolonged the accumulation of $\mathrm{p} 40 \mathrm{PX}-\mathrm{mCh}$ onto phagosomes, together the data suggest that luminal $\mathrm{pH}$ controls PtdIns(3)P depletion from the membranes of canonical phagosomes.

\subsubsection{Luminal $\mathrm{pH}$ between 6.1-6.3 controls PtdIns(3)P turnover on phagosomes}

Next, we were interested in identifying the luminal $\mathrm{pH}$ range at which PtdIns(3)P falls from the membrane of canonical phagosomes and see if it is similar to that of tubular phagocytic cups. To this end, we carried out internal calibration assays similar to those for tPCs. Using live-cell confocal microscopy, we tracked p40PX-mCh fate on pHRodo-zymosan phagosomes over time and then performed internal $\mathrm{pH}$ calibrations to each phagosome (Figure 3.8a). After grouping phagosomes positive and negative for $\operatorname{PtdIns(3)P~we~found~that~the~median~} \mathrm{pH}$ for $\mathrm{p} 40 \mathrm{PX}-\mathrm{mCh}$ positive and negative phagosomes was 6.7 and 5.5, respectively (Figure 3.8b). By examining the boundary of the standard deviation between these conditions, we estimate that phagosomes tend to become negative for the lipid between a $\mathrm{pH}$ of 6.1-6.3. 
Additionally, we tracked the live dynamics of p40PX-mCh and pHRodo-zymosan fluorescence over time in three individual phagosomes. We found that PtdIns(3)P accumulates onto newly formed phagosomes as they acidify to a $\mathrm{pH}$ of 6.5 and begins to dissociate as the $\mathrm{pH}$ decreases (Figure 3.8c). Indeed, PtdIns(3)P gradually disappeared below this $\mathrm{pH}$ until it could no longer be detected at $\mathrm{pH}$ 5.5. Consequently, our data thoroughly demonstrates that $\mathrm{pH}$ controls the lifespan of PtdIns(3)P in both tubular phagocytic cups and canonical phagosomes. 


\subsubsection{Vps34 association onto membranes controls PtdIns(3)P cellular levels}

\subsubsection{Luminal $\mathrm{pH}$ controls Vps34 association onto membranes}

We were interested in elucidating the mechanism by which $\mathrm{pH}$ could be coordinating the turnover of PtdIns(3)P, possibly impacting the association of synthesis and turnover machinery on the phagosomal membrane. Since PtdIns(3)P can be depleted from Vps34-inhibited tPCs and further stabilized by PIKfyve inhibition, we suspect $\mathrm{pH}$ is not regulating the association of turnover machinery. Rather, since we ablated PtdIns(3)P on tubular phagocytic cups when blocking the Class III PI3K Vps34, we investigated whether $\mathrm{pH}$ may play a role in its inactivation or association with the phagosomal membrane. The Vps34 complex binds to endosomal and phagosomal membranes through the association of the Vps15, Beclin-1 and UVRAG subunits (Backer, 2016; Rostislavleva et al., 2015). Thus, we investigated whether forced acidification or alkalization affected the association of these proteins and other endosomal markers to the phagosomal membrane.

First, we fed RAW cells with IgG-opsonized latex beads and synchronized phagocytosis through centrifugation. We then allowed IgG-Fc $\gamma$ receptor engagement and phagosome internalization for 5 minutes, prior to forced acidification. After, we bathed cells in acidified media ( $\mathrm{pH}$ 4.0) and allowed for phagocytosis to proceed for 20 minutes. We isolated and purified phagosomes containing latex beads through differential centrifugation on a sucrose gradient, as described in the methods section, and immunoblotted for Vps34, Vps15 and UVRAG. Interestingly, following exposure to acidic media all three components of the complex dissociated from the phagosomal membrane (Figure 3.9a, b) compared to resting cells. Conversely, when we forced alkalization using $\mathrm{NH}_{4} \mathrm{Cl}$ during phagocytosis, all three subunits were found to be intensely bound to the phagosomal membrane. We attempted to investigate the role of the V-ATPase 
through ConA treatment, however, pre-treatment greatly inhibited phagosome formation and sufficient phagosome isolation needed for western blot analysis (data not shown). In comparison, the association of late endosomal markers Rab7 and Raptor remained unaffected by acidic media and alkalization, suggesting that the $\mathrm{pH}$-mediated mechanism is specific to the PtdIns(3)P machinery and not due to non-specific effects of $\mathrm{pH}$ on protein-membrane interactions (Figure 3.9a, b).

Next, we investigated whether $\mathrm{pH}$ can affect the biochemical state of the Vps34 complex II, such as through regulating the phosphorylation state of the complex subunits. To investigate protein phosphorylation, we used Phos-tag mobility shift gels to resolve the phosphorylation forms of each subunit (Kinoshita, Kinoshita-Kikuta, Takiyama, \& Koike, 2006; Kinoshita, Takahashi, Takeda, Shiro, \& Koike, 2004). Interestingly, acidic media and alkalization affected the phosphorylation pattern of UVRAG species relative to the resting counterpart, but did not affect Vps34 and Vps15 phosphorylation levels (Figure 3.9c, d). These data are consistent with a model whereby Vps34 complex association may be controlled through luminal $\mathrm{pH}$ changes that alters UVRAG activity or association with the cytoplasmic leaflet of phagosomes, although the mechanism still isn't clear.

\subsubsection{Vps34 controls PtdIns(3)P cellular levels on endosomes}

Lastly, we speculated whether the $\mathrm{pH}-$ dependent dissociation of Vps34 from phagosomal membranes may also apply to endosomes and modulate cellular levels of PtdIns(3)P levels. To test this hypothesis, we bathed RAW cells in acidic media ( $\mathrm{pH} 4.0)$ and investigated the impact of the recruitment of PtdIns(3)P probe 2FYVE-GFP onto endosomes and total cellular levels of PtdIns(3)P. However, using the membrane permeant $\mathrm{pH}$-sensor, $\mathrm{pHRodo}-\mathrm{AM}$, we determined that 
forced acidification affects both cytosolic and endosomal pH (Figure 3.10a). Forced acidification did impact endosomal PtdIns(3)P, as 2FYVE-GFP dissociated from endosomal membranes in a time dependent manner. Additionally, we measured the cellular levels of PtdIns(3)P by metabolically labeling phosphoinositides. We incubated cells with ${ }^{3} \mathrm{H}-m y o$-inositol to label PtdInsPs, followed by lipid extraction and deacylation, where the amount of PtdIns(3)P species compared to total phosphatidylinositol was measured by flow scintillation. Forced acidification greatly decreased PtdIns(3)P levels, whereas, PtdIns $(4,5) \mathrm{P}_{2}$ suffered a smaller non-significant decrease. In comparison, alkalization using $\mathrm{ConA}$ and $\mathrm{NH}_{4} \mathrm{Cl}$ slightly augmented or preserved total PtdIns(3)P lipid levels in the cells (Figure 3.10c).

Altogether, our data demonstrate that endosomes and canonical and tubular phagosomes employ a proton gradient across their membranes to signal PtdIns(3)P depletion from their surface through the action of Vps34 complex II and its differential regulation by luminal $\mathrm{pH}$. This mechanism couples the slow acidification of the endosomal and phagosomal lumen with the loss of PtdIns(3)P from the maturing organelle. Conversely, due to the presence of the actin-jacket diffusion barrier, the changes in luminal $\mathrm{pH}$ are affected by proton leakage from the proximal area of the tPC, which allows for the maintenance of PtdIns(3)P on the tPC membrane regardless of the loss of other early endosomal makers like EEA1 and association of late endosomal markers like Rab7 and LAMP1. As the tPC becomes longer and seals into a phagosome, a proton gradient sufficient to signal for $\operatorname{Ptd} \operatorname{Ins}(3) \mathrm{P}$ is generated allowing for the continuation of canonical phagosomal maturation. In section 6.1, I present an overall model, discuss possible mechanisms controlling this pathway and suggest future directions to identify and further our understanding of the possible regulatory machinery. 
3.3 Figures 
Figure 3.1

a

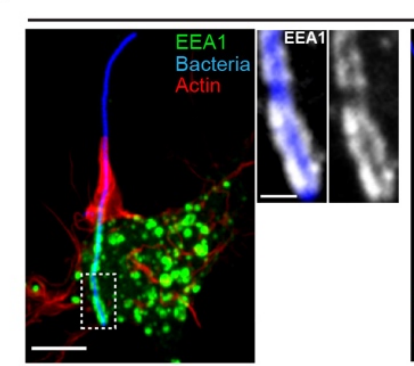

b

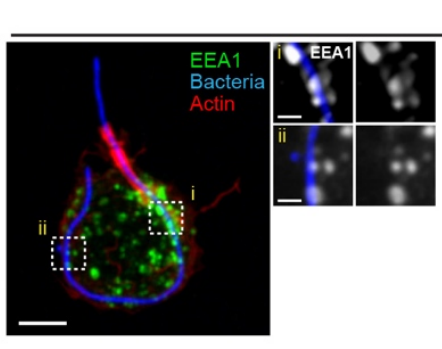

d

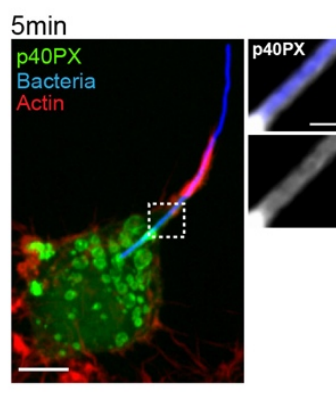

5 minutes

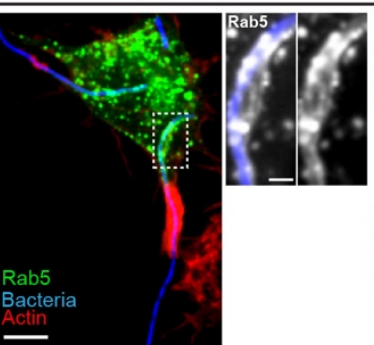

30 minutes
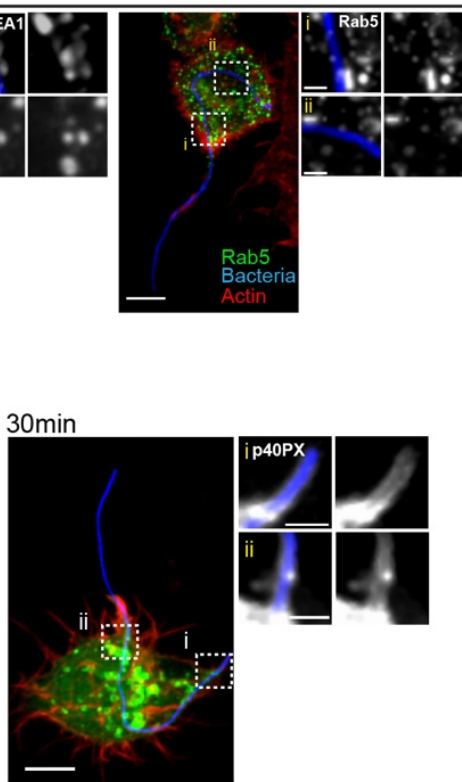

f
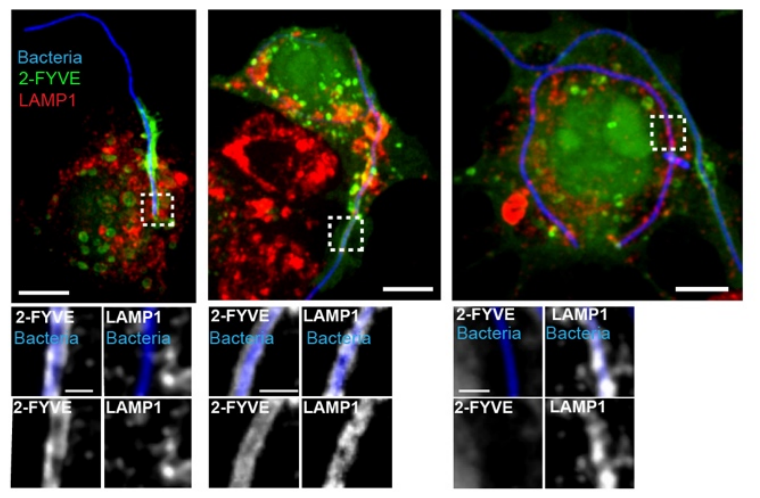

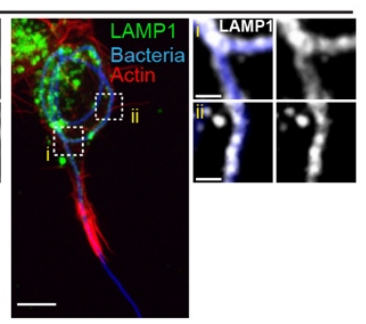

e

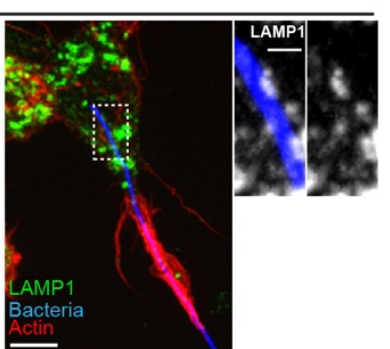

Bacteria

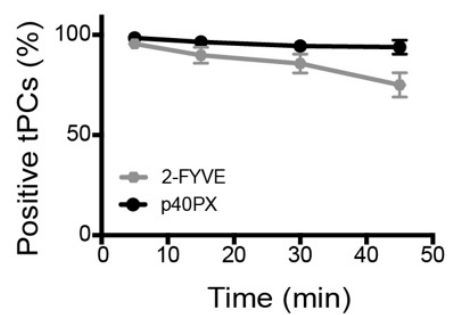

g

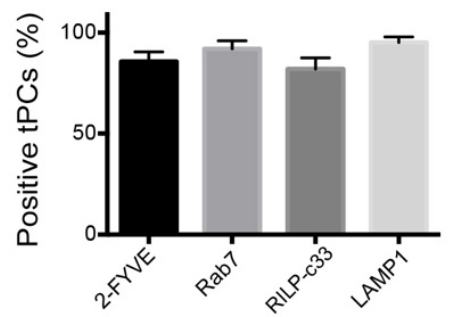

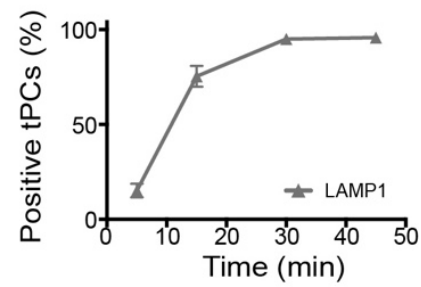


Figure 3.1: Non-canonical persistence of PtdIns(3)P on tPCs during maturation.

RAW macrophages challenged with f-LP overtime. (a, b) tPCs fuse with early (EEA1, Rab5) and late (LAMP1) endocytic compartments in a time-dependant manner. Actin jacket denoted by Factin accumulation (red). Images in main panels show merged z-stacks and smaller insets (right), show magnified single planes from framed regions showing marker recruitment. (c) Quantification of marker recruitment to tPC, determined positive if marker present throughout entirety of cup. (d, e) Recruitment and quantification of p40PX-GFP and 2FYVE-GFP to tPCs. (f, g) Recruitment of late endosomal markers (LAMP1, Rab7, RILPc33) to proximal and distal portions of tPC. (g) Number of tPCs positive for 2-FYVE, LAMP1, Rab7, and RILP-C33 recruitment at 30 min after the onset of phagocytosis. tPCs were scored positive for markers if labeling was observed along the entirety of the cup. Data shown are mean \pm SEM of percentages from three independent experiments ( $n=30-50$ at each time point). Scale bars are $5 \mu \mathrm{m}$ and $1 \mu \mathrm{m}$ for the enlarged areas. (Note: Figure experiment and data completed by Amriya Naufer). 
a

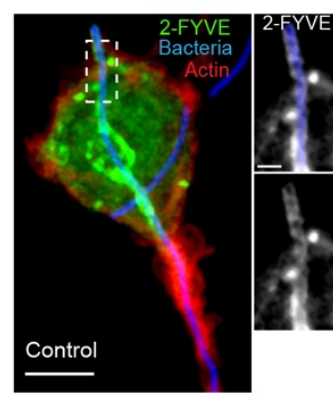

C

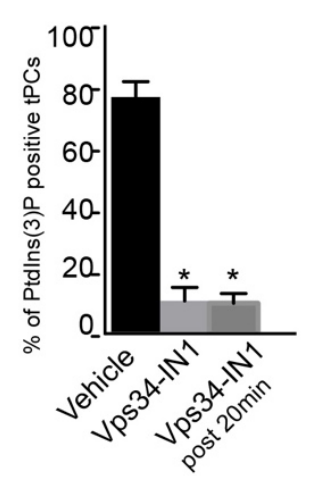

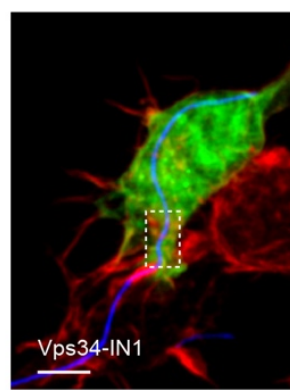

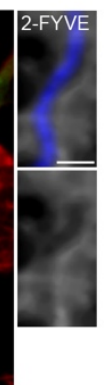

d

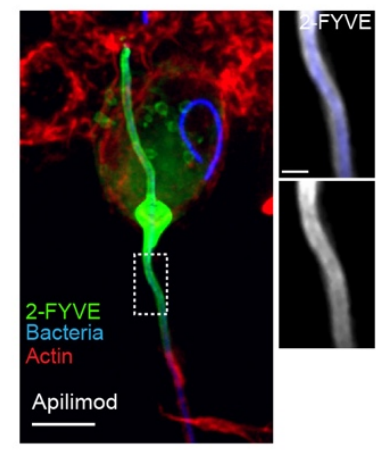

b
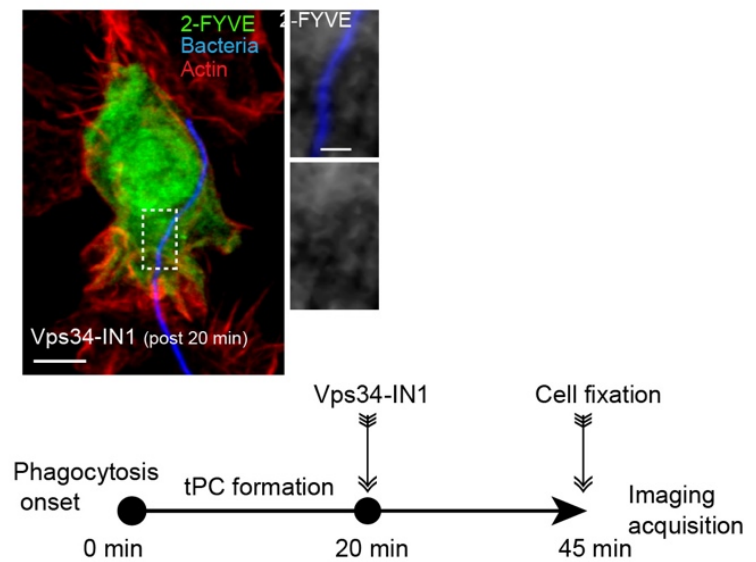

Figure 3.2: Vps34 activity is responsible for PtdIns(3)P persistence on tubular phagocytic cups.

RAW cells expressing 2FYVE-GFP (green) were treated with (a) DMSO (control) or $1 \mu \mathrm{M}$ VPS34-IN1 $1 \mathrm{~h}$ prior to the onset of phagocytosis or (b) 20 minutes following the onset of phagocytosis. (c) 2FYVE-GFP recruitment to tPCs were scored in all treatments. Data shown are mean \pm SEM from three independent experiments $(n=35$ for each). $\mathrm{p}<0.05$. Bars, $5 \mu \mathrm{m}$ and 1 $\mu \mathrm{m}$ for the enlarged areas. (d) Representative phenotype observed in cells pre-treated with $10 \mathrm{nM}$ apilimod, $1 \mathrm{~h}$ prior to and during phagocytosis. (Note: Figure experiment and data completed by Amriya Naufer). 
Figure 3.3

a
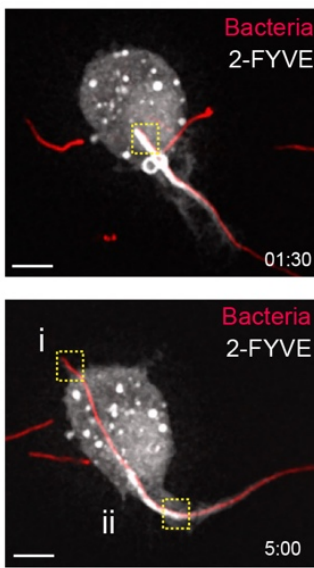

b
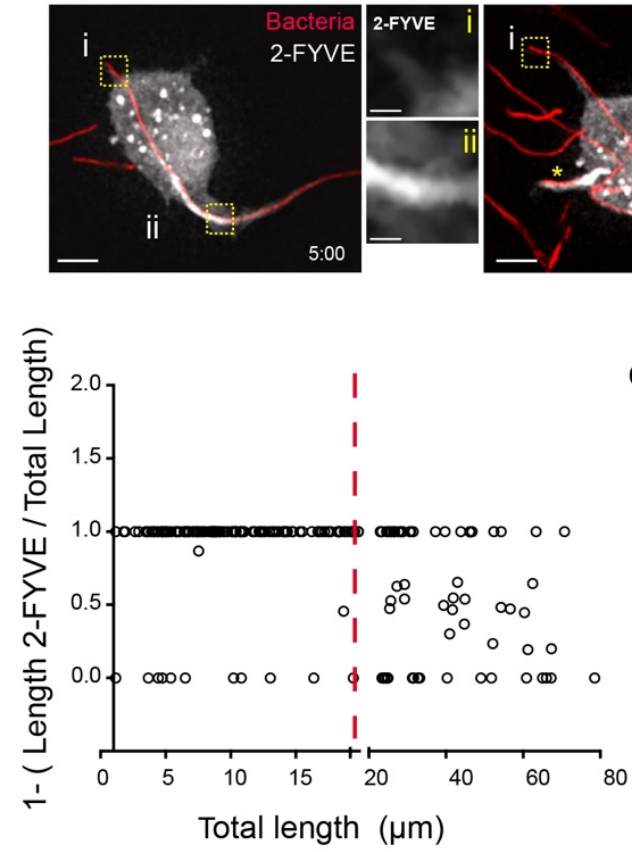
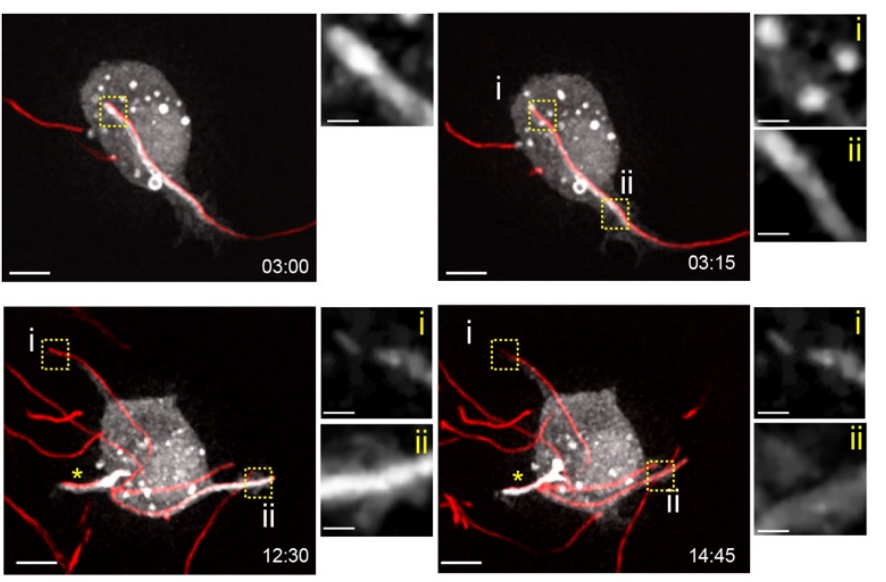

C

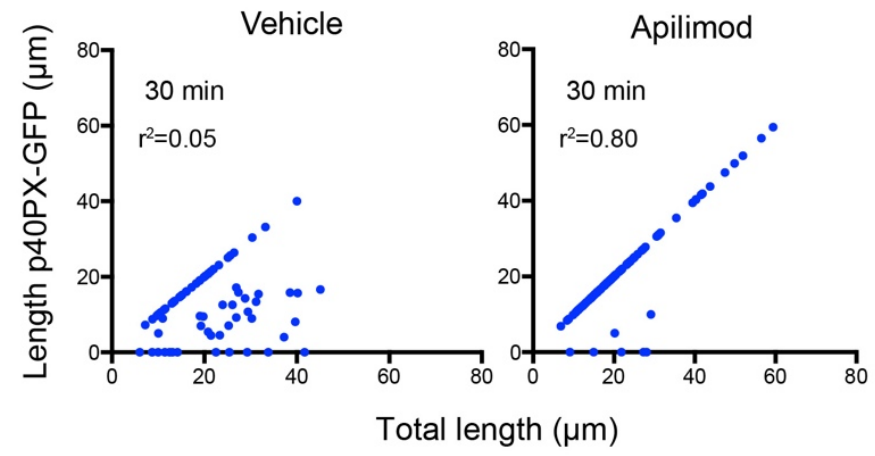


Figure 3.3: PtdIns(3)P dynamics during tPC internalization.

RAW cells expressing 2FYVE-GFP (white) were challenged with f-LP (red) over-time. (a) Selected frames of f-LP phagocytosis over time. Insets to the right of main panels are magnified regions highlighted in yellow. First two magnified-frames demarcate distal regions of tPCs, where, last four magnified-frames high-light (i) distal and (ii) proximal regions of tPC. Scale bars are 5 $\mu \mathrm{m}$ and $1 \mu \mathrm{m}$ in main panels and enlarged areas, respectively. (b) Regions of tPCs that surpass a length of $20 \mu \mathrm{m}$ (red line) tend to be devoid of 2FYVE-GFP. The ratio of 2FYVE-GFP-positive length to the total length of the IPC is shown. Ratio of 1 indicates that the entire tPC is decorated with 2 FYVE-GFP, ratio $<1$ indicates a portion is devoid of probe, and ratio of 0 indicates that the tPC is completely divested of probe. Data from three independent experiments $n=30$. (c) RAW macrophages expressing p40PX-GFP, pre-treated with DMSO (vehicle) or $10 \mathrm{nM}$ apilimod, internalized pHrodo-conjugated f-LP for 30 mins. The length of f-LP positive for $\mathrm{p} 40 \mathrm{PX}-\mathrm{GFP}$ was measured in 3 independent experiments ( $n=40$ for each condition) and plotted against total length internalized. The corresponding correlation coefficients $\left(\mathrm{r}^{2}\right)$ were calculated. (Note: a-b was completed by Amriya Naufer \& $\mathbf{c}$ by Victoria Hipolito). 
Figure 3.4

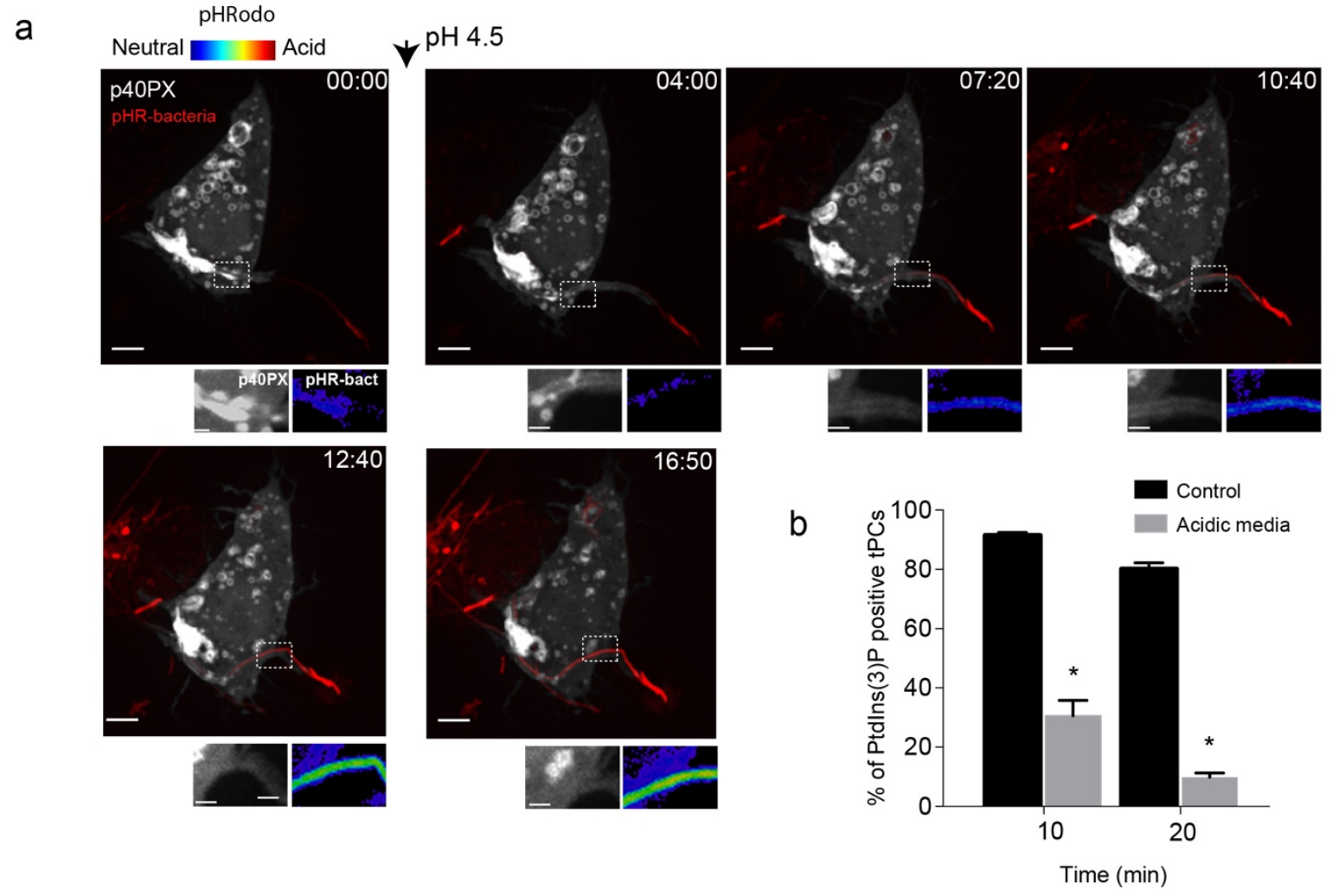

C
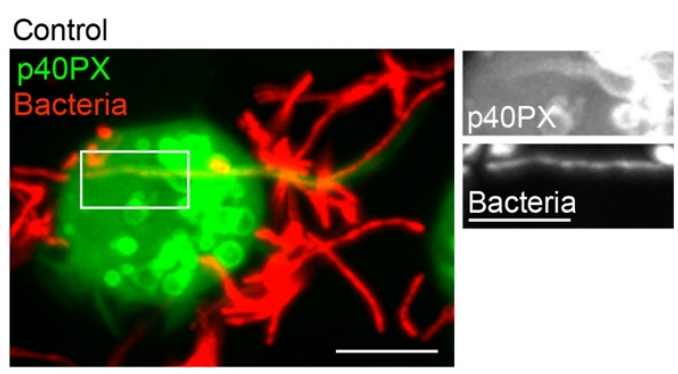

ConA

d
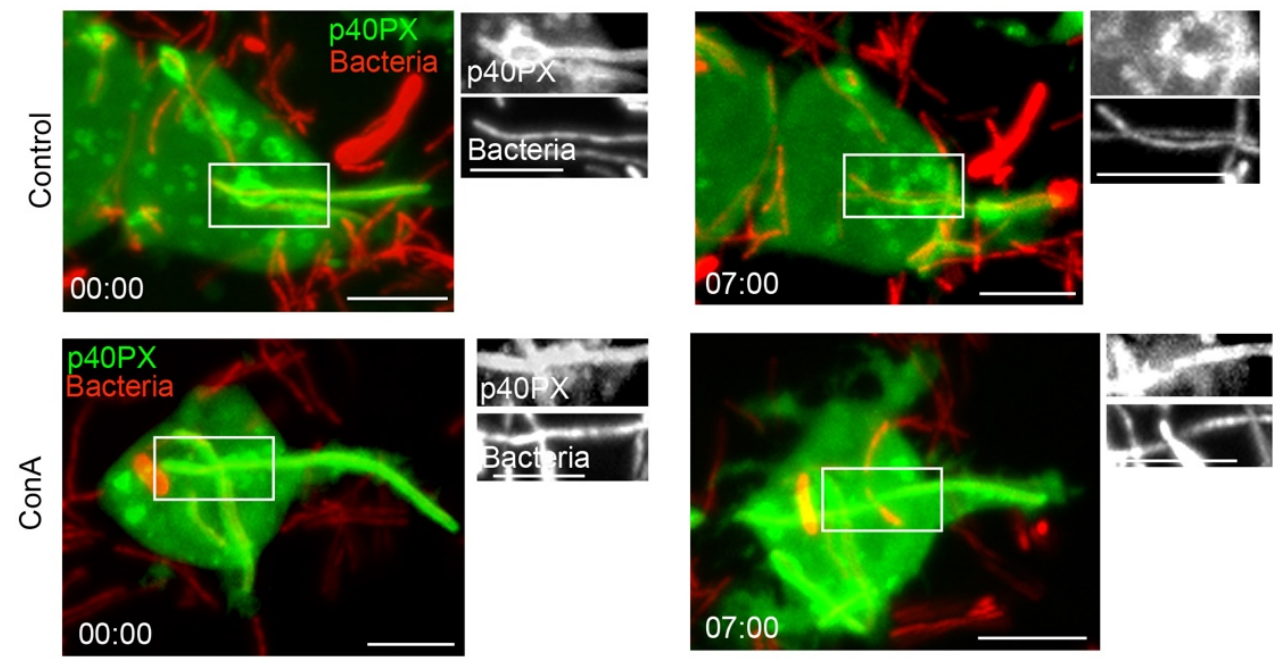
Figure 3.4: Luminal $\mathrm{pH}$ and V-ATPase activity control PtdIns(3)P dynamics on tPCs.

(a) Selected frames from a time-lapse sequence showing a RAW macrophage expressing p40PXGFP (white) engulfing a pHrodo-conjugated f-LP. Arrowhead indicates the time at which cells were exposed to culture media at $\mathrm{pH}$ 4.5. Images at the bottom of main panels show magnifications of the framed areas. Right panels show pHrodo signal (false-rainbow palette) and left panels show p40PX-GFP. Scale bars $=5 \mu \mathrm{m}$. (b) RAW cells expressing 2FYVE-GFP were challenged with $\mathrm{f}$ LP in neutral or acidic conditions. Following 10 and $20 \mathrm{~min}$ of phagocytosis, cells were imaged and quantified for the accumulation of 2FYVE-GFP at tPCs. Data shown are mean \pm SEM from three independent experiments $\left(n=30\right.$ for each, $\left.{ }^{*} \mathrm{p}<0.05\right)$. (c-d) RAW macrophages expressing p40PX-GFP (green) were left untreated or exposed to $1 \mu \mathrm{M}$ ConA before being challenged with f-LP (red). (c) Elongated tPCs remained labelled with p40PX-GFP in ConA-treated cells, while distal tPCs were divested of the probe in control cells. (d) In control cells, p40PX-GFP was recruited to tPCs and dissociated upon enclosure of the bacteria into a phagosome (marked at 7 min). In ConA-treated macrophages, phagosomes remained labelled with p40PX-GFP (marked at $7 \mathrm{~min})$. Scale bars $=5 \mu \mathrm{m}$. 

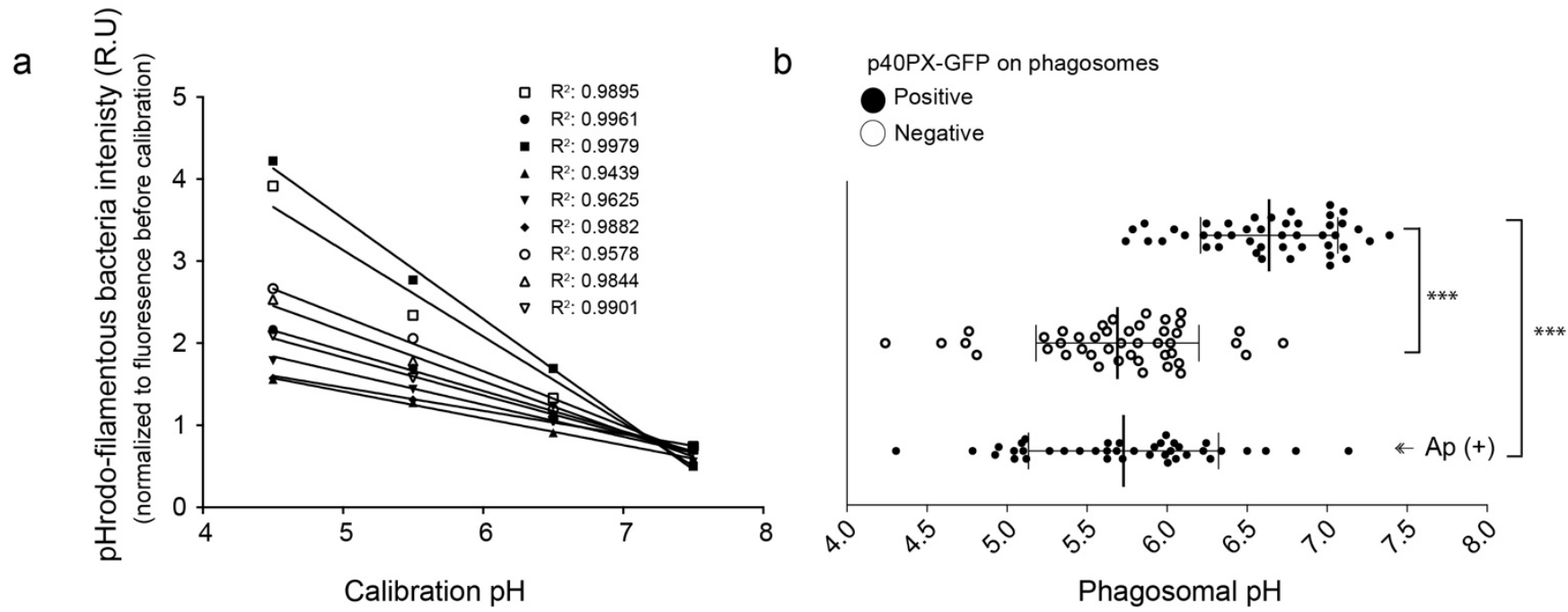

Figure 3.5: tPC luminal $\mathrm{pH}$ of 6.2 causes the dissociation of PtdIns(3)P probes.

(a) Representative $\mathrm{pH}$ calibration curves for 8 independent legionella-containing phagosomes. $\mathrm{pH}$ calibrations were performed as described in the materials and methods. The corresponding fluorescence emission for pHRodo-conjugated filamentous bacteria at each $\mathrm{pH}$, was normalized to the initial phagosome's fluorescence intensity (each value was corrected by background subtraction). The normalized values were plotted against their corresponding $\mathrm{pH}$ value and fit to a linear function, the corresponding $\mathrm{R}^{2}$ - values were determined. (b) RAW cells expressing p40PXGFP were challenged with pHrodo-conjugated filamentous bacteria. The phagosomal $\mathrm{pH}$ was determined for p40PX-GFP -positive and -negative phagosomes following 15 and 30 minutes of phagocytosis, respectively. $(\mathrm{Ap}+)$ Cells were treated with $10 \mathrm{nM}$ apilimod, 1 hour prior to phagocytosis and $\mathrm{pH}$ measured after 30 mins of phagocytosis. Data represents 15-20 phagosomal $\mathrm{pH}$ values $\pm \mathrm{SD}$ from three independent experiments. ANOVA test was employed to compare each group; $* * * p<0.001$. 


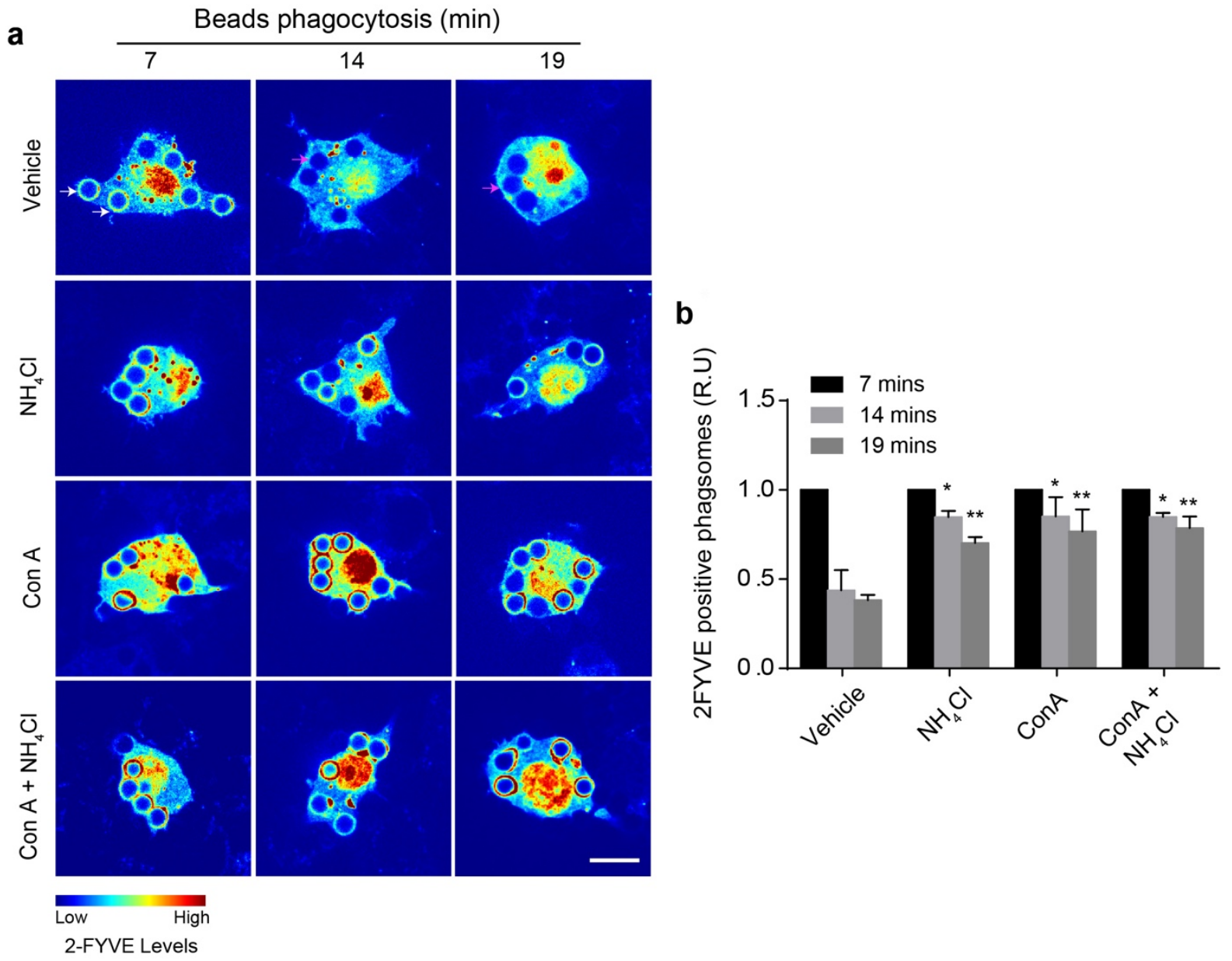

Figure 3.6: $\mathrm{pH}$ neutralization causes PtdIns(3)P to persists in phagosomes.

(a) RAW cells expressing 2FYVE-GFP (rainbow) were treated with either $0.1 \%$ DMSO (vehicle), $1 \mu \mathrm{M}$ concanamycin $\mathrm{A}$ (ConA), $10 \mathrm{mM} \mathrm{NH}_{4} \mathrm{Cl}$, or with both ConA and $\mathrm{NH}_{4} \mathrm{Cl}$ for $15 \mathrm{~min}$, prior to the phagocytosis of IgG-opsonized beads. White and magenta arrows depict examples of 2FYVE -positive and -negative phagosomes, respectively. (b) The phagosome: cytosol ratio of 2FYVE-GFP fluorescence for each time point indicated, normalized to $7 \mathrm{~min}$. Data shown are mean \pm SEM from three independent experiments ( $n=30$ for each). ANOVA test was employed to compare each treatment condition to vehicle, for each time point; ${ }^{*} \mathrm{p}<0.05$ and ${ }^{*} \mathrm{p}<0.01$. Scale bars $=5 \mu \mathrm{m}$. 
Figure 3.7

a
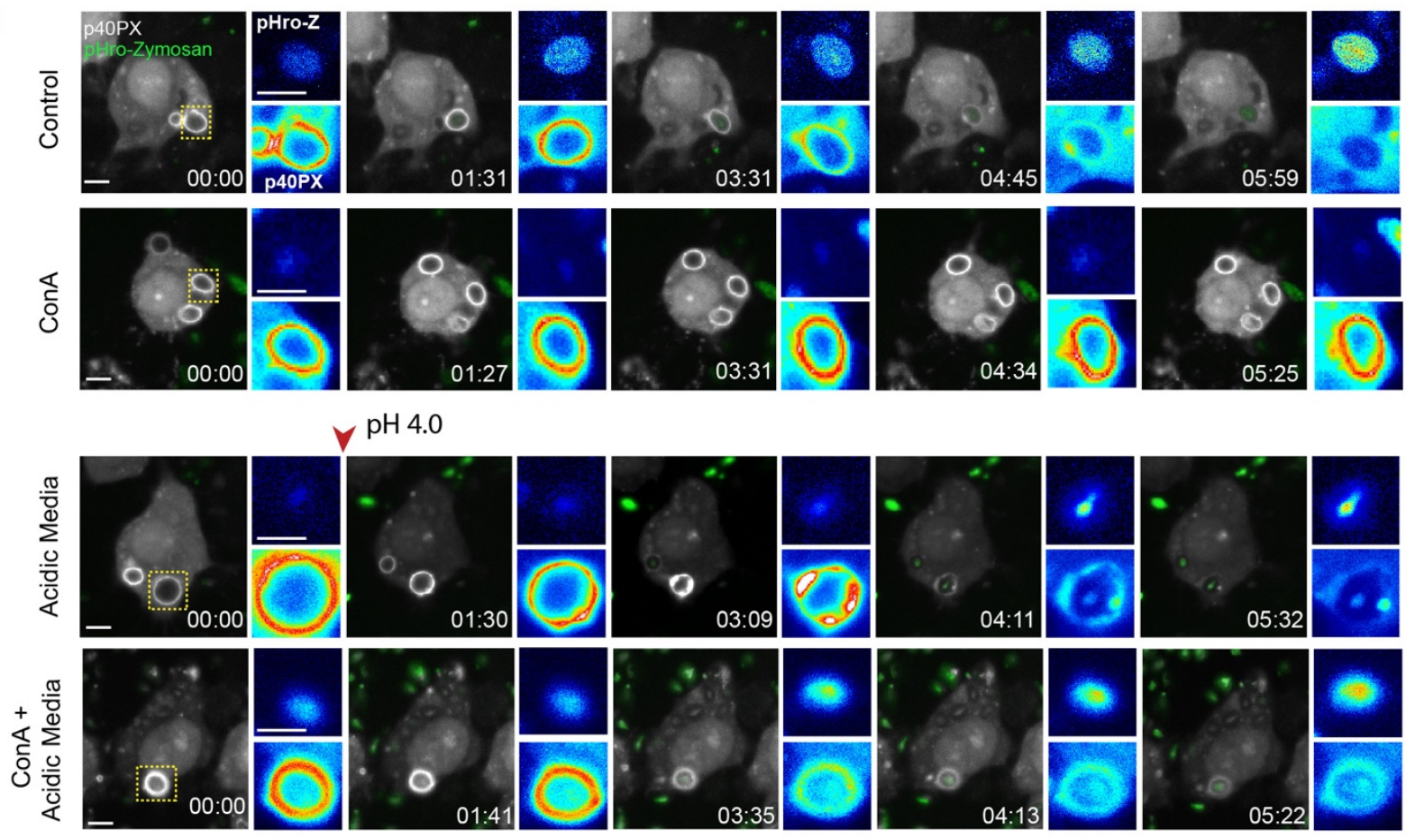

p40PX

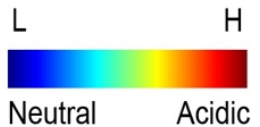

b
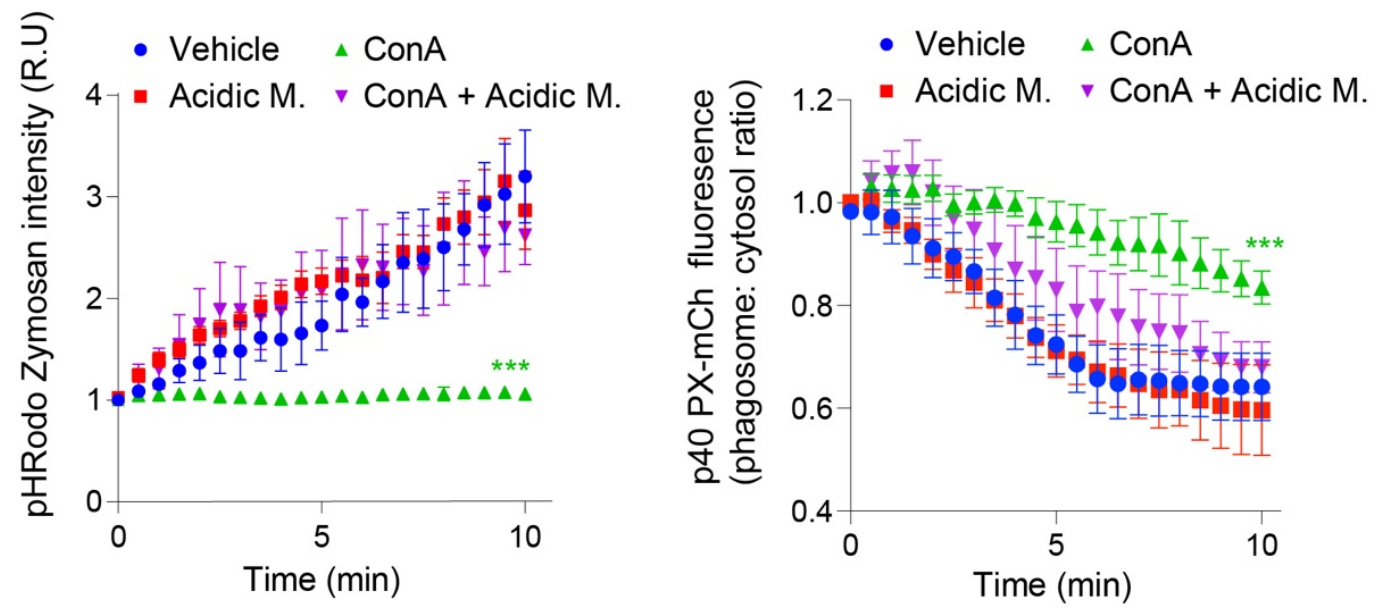
Figure 3.7: $\mathrm{p} 40 \mathrm{PX}-\mathrm{mCh}$ recruitment to zymosan-containing phagosomes is $\mathrm{pH}$-dependent.

(a) Live-cell imaging of p40PX-mCh-expressing (white) RAW cells, internalizing pHrodoconjugated zymosan particles (green), either in culture media (vehicle) or in acidic media (pH 4.0), with or without $1 \mu \mathrm{M}$ ConA. The arrowhead indicates the switch to acidic media, $30 \mathrm{~s}$ after the onset of the experiment. Images were acquired every 15-30 sec. Images to the right of the main panels show fluorescence intensities (rainbow) for pHrodo-zymosan (top) and p40PX-mCh (bottom) phagosomes framed in the main panels. Blue indicates low (L) fluorescence levels for p40PX-mCh and pHrodo (neutral $\mathrm{pH}$ ), while red indicates high $(\mathrm{H})$ emission signal for $\mathrm{p} 40 \mathrm{PX}-$ $\mathrm{mCh}$ and $\mathrm{pHrodo}$ (acidic pH). (b) Progression of pHRodo and p40PX-mCh fluorescence intensities in zymosan-containing phagosomes for the experimental conditions described in $a$. Data shown are normalized mean \pm SEM from 3 independent experiments ( $n=10-15$ phagosomes for each). ANOVA test was employed to compare each treatment condition to control; ***p $<0.001$. Scale bars $=5 \mu \mathrm{m}$. 
a

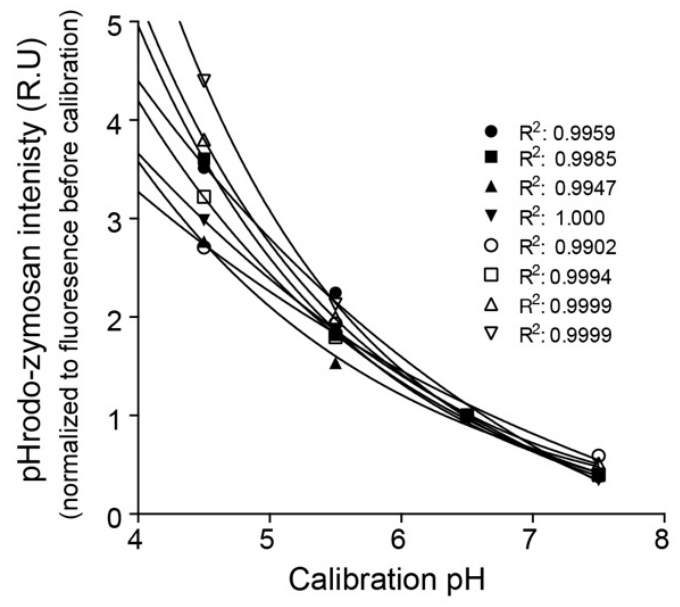

b

p40PX-mCh on phagosomes

C

- Positive

Negative
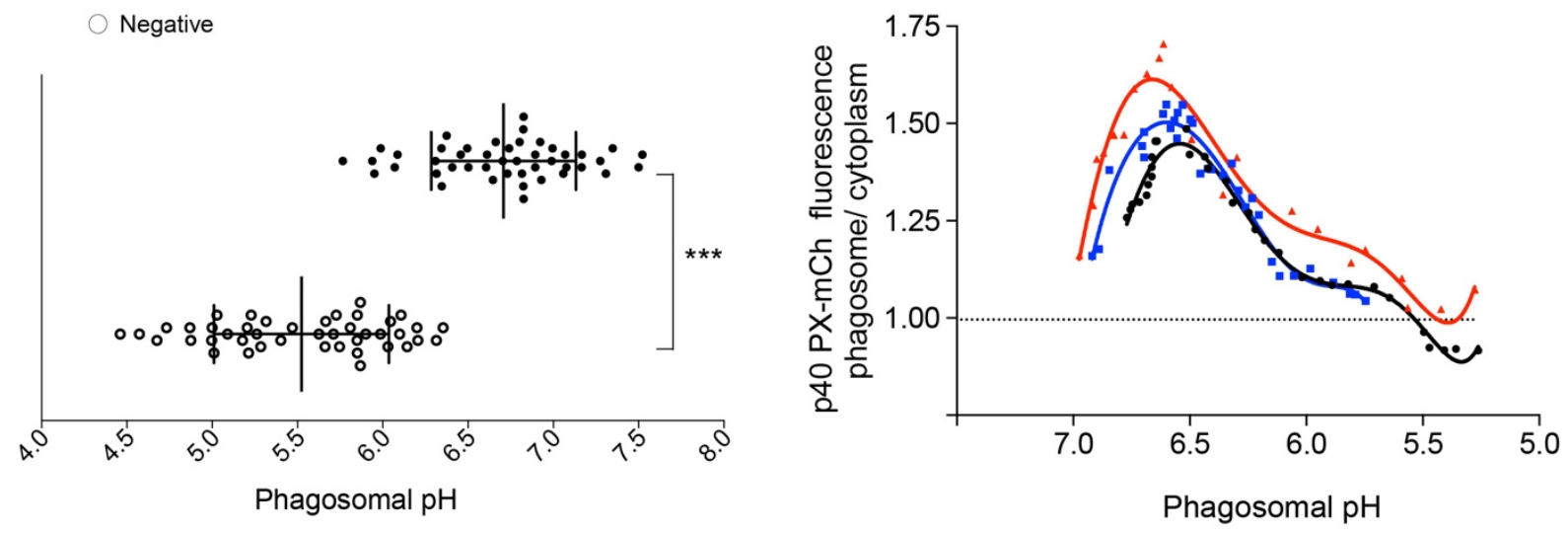

Figure 3.8: Phagosomal luminal $\mathrm{pH}$ between 6.1-6.3 causes dissociation of PtdIns(3)P probes.

(a) Representative $\mathrm{pH}$ calibration curves for 8 independent zymosan-containing phagosomes. $\mathrm{pH}$ calibrations were performed as described in the materials and methods. For zymosan-containing phagosome $\mathrm{pH}$ calibrations, each curve was fit to a one-phase exponential decay function, the corresponding $\mathrm{R}^{2}$ - values were determined. The function was used to determine the $\mathrm{pH}$ of the phagosome for that series. (b) RAW cells expressing p40PX-mCh were challenged with pHrodoconjugated zymosan and $\mathrm{pH}$ determined for $\mathrm{p} 40 \mathrm{PX}-\mathrm{mCh}$-positive and negative phagosomes. Data represent 15 phagosomal $\mathrm{pH}$ values $\pm \mathrm{SD}$ from three independent experiments, each phagosome was internally calibrated $(* * * \mathrm{p}<0.001)$. (c) Relationship between phagosomal $\mathrm{pH}$ and $\mathrm{p} 40 \mathrm{PX}-\mathrm{mCh}$ in zymosan-containing phagosomes. Data represents three independent phagosomes followed over 30-40 second intervals for 15 minutes. 
Figure 3.9
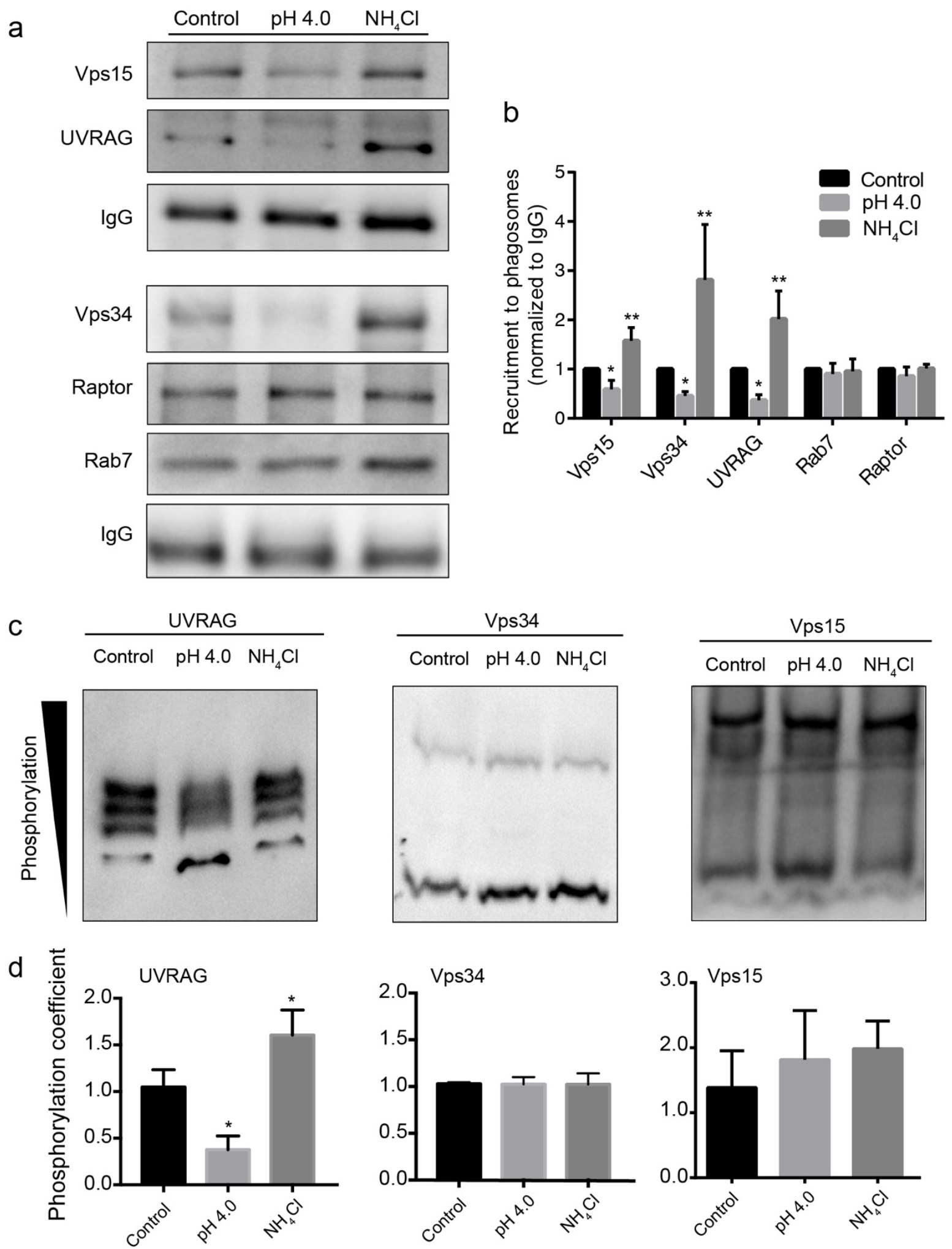
Figure 3.9: The Vps34 complex II association to phagosomes is controlled by $\mathrm{pH}$.

(a) Vps34 complex II subunits associated to isolated latex beads phagosomes. RAW macrophages either in culture media (control), acidic culture media ( $\mathrm{pH} 4.0$ ) or cell-culture medium with $10 \mathrm{mM}$ $\mathrm{NH}_{4} \mathrm{Cl}$ were allowed to internalize IgG-opsonized latex beads for $20 \mathrm{~min}$. After this period, phagosomes were isolated and immunoblotted for the presence of Vps15, UVRAG, Vps34, Raptor, and Rab7. Human IgG was employed as a control for number of phagosomes loaded. (b) Protein densitometric quantification for blots shown in $a$. Human IgG was used to normalize phagosomal loading. Data shown are mean \pm SEM from four to six independent experiments; ${ }^{*} p<0.05$ and ${ }^{* *} \mathrm{p}<0.01$. (c) Phos-Tag SDS-PAGE determination of the phosphorylation states of UVRAG, Vps34 and Vps15 in phagosomes isolated as described in $\boldsymbol{a}$. (d) The phosphorylation coefficient was used to quantify the preferred states of phosphorylation. This was done by calculating an internal ratio of the most (top) to least (bottom) phosphorylated species. Data shown are mean \pm SEM from three or four independent experiments * $p<0.05$. 
Figure 3.10

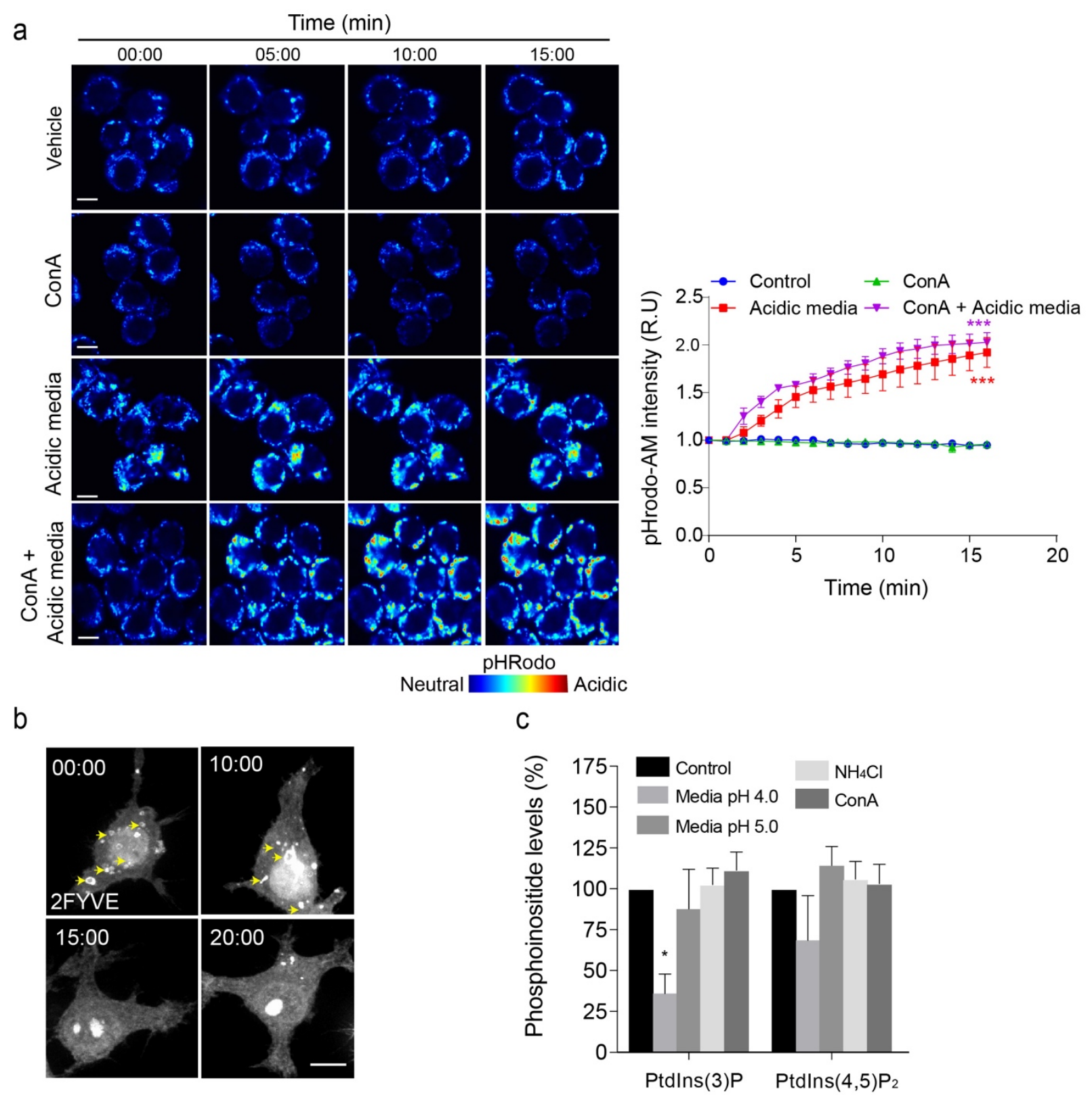


Figure 3.10: PtdIns(3)P on endosomes is controlled by $\mathrm{pH}$.

(a) The cytoplasmic $\mathrm{pH}$ of pHrodo-AM-loaded RAW macrophages over time. RAW macrophages were incubated in either culture media (control), or acidic media ( $\mathrm{pH} 4.0$ ), with or without $1 \mu \mathrm{M}$ ConA. pHrodo-AM fluorescence intensity is shown in rainbow pallet. Scale bars $=5 \mu \mathrm{m}$. Left, changes in fluorescence intensity over time. Data shown are mean \pm SEM from three independent experiments ( $\mathrm{n}>50$ cells for each condition). ANOVA test was employed to compare each treatment condition to control; $* * * \mathrm{p}<0.001$. (b) RAW macrophages expressing $2 \mathrm{FYVE}-\mathrm{GFP}$ (white) show PtdIns(3)P containing endosomes (arrows). 2FYVE-GFP positive endosomes disappeared when macrophages were exposed to acidic cell-culture medium $(\mathrm{pH} 4.0)$ for the time points indicated (representative of 3 independent experiments). Scale bars $=5 \mu \mathrm{m}$. (c) Determination of PtdIns(3)P level in RAW macrophages. Cells were incubated with ${ }^{3} \mathrm{H}-m y o-$ inositol overnight and subjected to the conditions indicated for $20 \mathrm{~min}$. Following inositol isolation and separation, levels of PtdIns(3)P and PtdIns(4,5) $\mathrm{P}_{2}$ were determined. For each treatment, PtdInsP levels were normalized to the parent PtdIns peak and compared to control. Data shown are mean \pm SEM from four independent experiments; ${ }^{*} p<0.05$. 
Chapter 4: Lysosome adaptation and remodelling in activated phagocytes 


\subsection{Chapter rationale and overview}

Organelle compartmentalization helps a cell maintain cellular order and function. Organelles can exist in a range of morphologies optimal for function, such as from individual vesicular organelles to a continuous membrane reticulum. Despite this complexity, cells must control organelle number, size, shape and activity to meet the needs of their differentiation state. In addition, cells must adapt these organellar properties in response to intrinsic and extrinsic stimuli that alter the metabolic and functional needs of cell (Behnia \& Munro, 2005; Chan, et al., 2016; Levy \& Heald, 2012; Mills \& Taghert, 2012; Mullins \& Bonifacino, 2001). Immune cells like macrophages and dendritic cells are highly plastic as they can adopt "resting", highly inflammatory, and antiinflammatory states that differ in their gene expression profiles, metabolic programming, secretory pathway activity and endolysosomal membrane system (Hipolito, et al., 2018; Janssens, et al., 2014; Kelly \& O’Neill, 2015; Porta, et al., 2015; Trombetta, et al., 2003).

The endo-lysosomal systems of phagocytes undergo striking remodelling upon lipopolysaccharide (LPS) activation through the phosphatidylinositol-3-kinase-AKT-mTOR signalling axis, converting dozens of individual globular lysosomes into a striking tubular network (Hipolito et al., 2018; Mrakovic et al., 2012; Vyas et al., 2007). Although lysosome tubulation is associated with the retention of pinocytic cargo, exchange of phagosomal cargo, and possibly antigen presentation (Boes et al., 2002; Chow et al., 2002; Mantegazza et al., 2014; Nakamura et al., 2014; J Swanson et al., 1987), it is not known how lysosome tubulation helps phagocytes function in response to stimuli.

Lysosomes also serve as signalling platforms that sense the metabolic and nutrient states of a cell through the activity of mTORC1 (Jewell, Russell, \& Guan, 2013; Lim \& Zoncu, 2016; Mony, Benjamin, \& O’Rourke, 2016). Active mTORC1 phosphorylates a variety of downstream 
targets to stimulate anabolic pathways, including mRNA translation (Buszczak, Signer, \& Morrison, 2014; Thoreen, 2017; Zoncu, et al., 2011) and repression of catabolic processes including autophagy (Ganley et al., 2009; Jung et al., 2009; Roczniak-Ferguson et al., 2012; Settembre et al., 2012). mTORC1 also plays roles beyond coordinating anabolism and catabolism, such as responding to Toll-like receptor ligands like LPS, Salmonella invasion, phagocytosis and inflammasome activators (Gray et al., 2016; Moon et al., 2015; Owen, Meyer, Bouton, \& Casanova, 2014; Saric et al., 2016), though the functional consequences of this phenomenon are not entirely clear.

In this chapter, I set out to investigate the mechanisms underlying the remodelling and reorganization of the endo-lysosomal system of phagocytes in response to LPS stimulation. Firstly, I determined that LPS-activated phagocytes increase their total lysosome volume and retention capacity. This expansion required de novo protein synthesis of key lysosomal proteins independent of transcriptional mechanisms and the action of the MiTF/TFE transcription factor family. We found instead that mTOR-dependent selective and enhanced translation of lysosomal transcripts drove lysosomal expansion and bolstered antigen presentation and T-cell activation. 


\section{$4.2 \quad \underline{\text { Results }}$}

\subsubsection{Acute LPS-activation of phagocytes remodels the lysosome population}

\subsubsection{Activation of macrophage and dendritic cells expands lysosome volume}

The phenotype of lysosome tubulation observed in phagocytes like DCs and macrophages stimulated with LPS is striking. We were interested in investigating if tubulation is coupled with an increase in lysosomal volume considering the vast reorganization of the lysosomal network. To investigate this, we employed several cell models: primary macrophages and DCs isolated from murine bone-marrow and the RAW macrophage cell-line. To this end, we pre-labelled lysosomes by feeding the cells fluid phase fluorescent marker Alexa546-conjugated dextrans, and then exposed cells to LPS or vehicle for $2 \mathrm{~h}$ to induce tubulation (Figure 4.1a). Pre-labelling cells prior to stimulation ensured that lysosomes were equally loaded with the dye in resting and activated cells. Using live-cell confocal fluorescent microscopy, we estimated the total change in lysosomal volume through three-dimensional volumetric analysis tools by comparing the total amount of fluorescent voxels in resting and activated cells. Compared to their resting counterpart, we saw a near two-fold significant increase in total lysosomal voxel count in bone-marrow derived DCs (BMDC) and macrophages (BMDM), and the RAW cell-line following LPS stimulation (Figure 4.1b).

Mrakovic et al. (2012) previously showed that tubular lysosomes are more dynamic than punctate lysosomes. In order to control for the displacement of tubules during Z-stack acquisition, we employed the same volumetric analysis in fixed cells. Though common, paraformaldehyde (PFA) fixation is well-known to break down lysosome tubules (Figure 4.1c, d), therefore we optimized a mixed-formaldehyde fixation technique that better preserved lysosomal tubules. The combination of low-percent glutaraldehyde and PFA maintained the population of cells that 
tubulated in response to LPS, when comparing to live-cells (Figure 4.1c, d). We then employed the same volumetric analysis in fixed RAW cells, where LPS stimulated cells maintained the near two-fold increase in lysosome volume compared to resting cells (Figure 4.1e). Additionally, we also measured the total lysosomal volume in fixed primary macrophages with the standard PFA protocol and the increase in total lysosomal volume was preserved (Figure 4.1f), further supporting an increase in luminal volume regardless of shape.

Furthermore, to circumvent the possibility that the limit of resolution attributed to spinningdisc confocal microscopy might contribute to the increase in lysosome volume, we employed structured illumination microscopy (SIM) which enables imaging in super-resolution (Gustafsson, 2005). Due to the limitations of the system (i.e. timing of acquisition and sensitivity of camera) we were unable to employ volumetric analysis, so instead we sampled three z-slices on the $\mathrm{x}-\mathrm{y}$ plane at the mid-points of each cell and quantified the area occupied by the fluorescent-fluid phase marker following super-resolution image reconstruction (Figure 4.2a). The super-resolution system also showed an increase in total lysosomal fluorescent area that was sampled in BMDM, BMDC and RAW cells, when comparing to resting counterparts (Figure 4.2b). Altogether, our data supports LPS-activated lysosomal tubulation is coupled with an increase in total lysosomal volume.

\subsubsection{Phagocyte activation increases lysosomal holding capacity}

One of the hallmark functions of phagocytes is to sample tissues through macropinocytosis for invading particles or to internalize them through receptor-mediated phagocytosis. Increasing lysosomal volume may therefore help to facilitate phagocyte uptake and holding capacity. Therefore, we speculated whether LPS activated phagocytes have higher lysosomal holding 
capacities. We incubated cells with lucifer yellow, a small molecular weight fluid-phase marker, and tracked its uptake to saturation in activated cells. We saw that both RAW and primary macrophages pre-activated with LPS exhibited a large increase in fluid-phase accumulation relative to resting cells at each time point (Figure 4.3a, b \& Figure 4.4a). Additionally, we tracked the rates of endocytosis by following the early accumulation of lucifer yellow. We found that preactivated primary macrophages displayed faster rates of pinocytic uptake relative to resting cells (Figure 4.3c). Interestingly, the rate of uptake was amplified within 15 minutes of LPS exposure, as indicated by macrophages concurrently undergoing pinocytosis and stimulation (Figure 4.3c). Next, we investigated whether activated cells recycle fluid-phase material at different rates compared to resting cells. To test this, we pre-activated macrophages with LPS for two hours, as in (Figure 4.3c), then introduced lucifer yellow in culture medium for 30 minutes. After internalization, we chased the probe over time in probe-free medium and followed its retention. Interestingly, there was no difference in the rate of probe depletion in pre-activated cells compared to resting cells, suggesting that rates of exocytosis were similar for both (Figure 4.3d).

Similarly, we followed the accumulation and rates of endocytosis and exocytosis of lucifer yellow in RAW macrophages. As seen with primary cells, RAW macrophages pre-stimulated with LPS or in combination with lucifer yellow uptake, accumulated more of the dye over time (Figure 4.4a). When measuring early endocytic rates, RAW cells demonstrated slightly different dynamics, where addition of LPS during LY uptake did not augment LY levels. However, after 30 minutes of uptake in the pre-treated group, we began to see an enhancement in uptake, suggesting that RAW macrophages likely required more time to bolster trafficking (Figure 4.4b). Similar to primary macrophages, exocytic rates in resting and the LPS pre-treated groups did not differ 
(Figure 4.4c). Collectively, the data in both primary and immortalized cells indicate that activated macrophages had a higher lysosome holding capacity relative to resting macrophages.

Moreover, we were curious if primary dendritic cells would benefit from increased lysosomal holding capacity, considering that DCs are professional antigen presenting cells and rely on the lysosomal network for antigen processing and presentation. Interestingly, the literature supports the idea that following dendritic cell activation and maturation, dendritic cells downregulate their endocytic capacity (Garrett et al., 2000). However, the majority of research follows DC endocytic capacity following 16 hours of maturation and fails to examine earlier time points. Therefore, we speculate that early activation of DCs may bolster lysosome remodelling and holding capacity, and determined that DCs activated with LPS maintain their endocytic capacities up to 8 hours post-activation (Figure 4.4d). The data suggest that DCs can increase pinocytic uptake and lysosomal volume early during activation to facilitate antigen uptake. Overall, our observations are consistent with previous reports suggesting that tubulation in activated macrophages may aid in retaining fluid phase material and that mature dendritic cells continue to engulf extracellular material (Drutman \& Trombetta, 2010; Platt et al., 2010; Swanson et al., 1985; Swanson, Burke, \& Silverstein, 1987). 


\subsection{2 mTOR modulates LPS-induced lysosome remodelling}

\subsubsection{Activated macrophages express higher levels of lysosomal proteins}

Our data demonstrate that lysosomes stimulated with LPS for 2 hours, a shorter time in comparison to most publications studying immune activation, caused lysosomes to reorganize into a tubular network, increasing total lysosomal volume and holding capacity. Henceforth, we call the structural and resulting functional reorganization of the lysosome network "lysosomal remodelling". Next, we sought to understand what mechanisms might contribute to the remodelling process. We postulated that lysosomal biogenesis is enhanced to accommodate for the structural reorganization and expansion.

To investigate this, we first measured the amount of endogenous LAMP1-positive structures in LPS stimulated cells, as a marker for the expansion of the lysosomal network. We saw a time-dependent increase in total LAMP1 structures per micron ${ }^{3}$, indicating an increase in total protein level regardless of cell size (Figure 4.5a, b). Next, we probed for the levels of the key lysosomal markers LAMP1, LAMP2, CD63, cathepsin D, and D and H subunits of the V1 VATPase complex in resting cells and in cells stimulated with LPS. Interestingly, we saw a significant increase in LAMP1, LAMP2, ATP6V1H, ATP6V1D, CD63 and mucolipin-1 protein levels following long-term stimulation (6 h) (Figure 4.5c, d; Figure A4), supporting previous literature (Pastore et al., 2016). When we activated macrophages with LPS for 2 hours, corresponding to when we observed an increase in lysosome tubulation and volume (Mrakovic et al., 2012; Saric et al., 2016; Figure 4.1), we saw a significant 2-fold increase in the aforementioned protein targets. Interestingly, if we activated cells with LPS for 2 hours and chased for 4 hours without LPS, the increases in lysosomal protein levels were maintained (Figure 4.5c, d; Figure A4). These data suggest that pre-stimulated cells retain the capacity to remodel their lysosomes, 
which parallels with the increased lysosomal holding capacity observed following pre-activation (Figure 4.3a-c \& Figure 4.4a, b). In all cases cathepsin D levels were unchanged, suggesting that LPS may exert differential effects on lysosomal proteins.

Next, we speculated that protein synthesis may be driving the increase in lysosomal proteins, so we treated cells with the translation elongation inhibitor cycloheximide. The increase in LAMP1, LAMP2, ATP6V1D, ATP6V1H, CD63 and mucolipin-1 protein levels was blunted, suggesting that de novo protein synthesis, rather than reduced protein turnover, augments lysosomal protein levels during LPS stimulation (Figure 4.5c, d; Figure A4). Moreover, cycloheximide treatment also blunted lysosomal tubulation and expansion in macrophages cotreated with LPS (Figure 4.5e, f). Together, our data suggest that LPS augments de novo protein synthesis to drive lysosome expansion and remodelling during phagocyte activation.

\subsubsection{Rapid lysosome expansion is not dependent on TFEB and TFE3}

Our results indicate that lysosome biogenesis plays a role in lysosomal remodelling during phagocyte activation. The MiTF/TFE transcription factor family responds to environmental stressors or signals such as starvation, phagocytosis and immune stimulation to scale up lysosomal biogenesis (Gray et al., 2016; Martina et al., 2014; Pastore et al., 2016; Settembre et al., 2012). Therefore, we investigated whether TFEB and TFE3, the most-studied of the family members, respond to LPS in activated phagocytes to drive lysosome biogenesis and remodelling through enhanced transcription of lysosomal genes.

We first probed for the localization of endogenous TFEB and TFE3 in activated and resting cells. Consistent with the literature, TFEB and TFE3 both undergo nuclear localization in response to long-term LPS (6 h) stimulation and upon treatment with Torin1 (Figure 4.6a, b). Interestingly, 
though LPS activation following 2 hours of stimulation induces lysosome remodelling, TFEB and TFE3 did not undergo nuclear translocation, consistent with the delayed response observed in Pastore et al (2016). Moreover, when we probed for the transcript levels of known lysosomal gene targets of TFEB and TFE3 (i.e. LAMP1, ATP6V1D, ATP6V1H and TRPML1), we did not observe changes in their mRNA levels, even following long-term stimulation (Figure 4.6c). In contrast, there was a substantial increase in the amount of interleukin- 6 mRNA, suggesting LPS is activating immune signalling (Figure 4.6d).

We obtained TFEB and TFE3 single and double knock-out RAW macrophage cell lines, to exclude the role these proteins may play in regulating lysosome remodelling (Figure A5a, b). To employ our lysosomal remodelling assays, we investigated if the absence of these two proteins impacted basal lysosomal number and membrane trafficking. First, we measured the levels of LAMP1 in the single and double knock-out cell lines, and saw no difference in overall expression, indicating that basal lysosomal homeostasis is likely not impacted (Figure A5c, d). Next, we determined if fluid-phase uptake and trafficking to lysosomes was impacted in these cells by quantifying the degree of co-localization between LAMP1-positive vesicles and fluorescentdextran. Using Mander's overlap coefficient, we measured no overall difference in the mutant lines compared to wild-type, suggesting that basal trafficking is not impacted (Figure A5e, f). Moreover, we measured the accumulation of fluorescent-dextran in cells following a 1 hour pulse and 1 hour chase assay, to detect possible differences in dye loss, and determined there was no difference in the mutant lines compared to resting cells (Figure A5d). Finally, to measure the possible effects LPS may have on subsequent dye trafficking following lysosome labeling with a pulse-chase assay, we measured the accumulation of fluorescent dextran following LPS and reproducibly observed no differences between dye fluorescence in the LPS and no LPS control, when compared 
to wild-type cells (Figure A5h). Together, our data demonstrate that the absence of TFEB and/or TFE3 has minimal impact on basal lysosomal biogenesis, pinocytosis and trafficking to lysosomes.

We then labeled lysosomes in wild-type and knock-out cells, and measured lysosome remodelling following acute LPS exposure. Interestingly, the lysosome population in both the single and double mutant forms of TFEB and TFE3 underwent tubulation comparable to the wildtype cells following LPS exposure (Figure 4.6e). Additionally, consistent with wild-type cells, lysosomes within cells lacking TFEB and/or TFE3 underwent expansion following LPS (Figure 4.6f). Overall, our data unexpectedly suggests that TFEB and/or TFE3-dependent transcription is not a major driver for the rapid lysosome expansion during acute macrophage activation.

\subsubsection{Rapid lysosome expansion depends on AKT and mTOR activity}

Since we did not see an increase in lysosomal mRNAs, but protein levels increased in response to LPS, we were interested in investigating whether translation may regulate lysosomal remodelling. It is well-established that activated macrophages undergo metabolic reprogramming, enhance protein synthesis, activate the unfolded protein response pathway and translation of mRNAs encoding inflammatory proteins (Janssens et al., 2014; Kelly \& O’Neill, 2015; Schott et al., 2014). We speculated that mTOR activity, a known regulator of mRNA translation and lysosome tubulation, is necessary for lysosome holding capacity (Buszczak et al., 2014; Saric et al., 2016; Thoreen, 2017).

First, we probed for the activation states of known regulators of translation that are mTOR downstream targets, S6K and 4E-BP1. The levels of both mTOR-targeted phosphorylation sites, Thr389 and Thr37/46 of p70S6K and 4E-BP1, respectively, increased following 2 and 6 hours of LPS stimulation. In the presence of the mTOR active-site inhibitor Torin1, the phosphorylation 
levels were blunted (Figure 4.7a, b) supporting a model that mTOR acts on these translational regulators in activated phagocytes.

As expected, when we directly blocked mTOR activity using Torin1, or indirectly through inactivating its upstream regulator, AKT, we blocked lysosomal tubulation (Saric et al., 2016; Figure 4.7c). Consistently, when we measure lysosomal expansion both AKT and mTOR activity were needed to augment lysosomal volume upon LPS stimulation (Figure 4.7d). We attempted to measure lysosomal protein levels upon LPS stimulation in mTOR-inhibited conditions, but observed varying effects on protein levels which may be explained by the confounding effects of slightly inducing autophagy in macrophages treated with Torin1 (Figure A4; Figure A6a, b). However, when we measured lysosomal protein levels in AKT-inhibited cells stimulated with LPS, the increase in lysosomal protein levels observed in LPS-treated cells was blocked (Figure 4.7e; Figure A4). Finally, we also probed the effects of mTOR inhibition on lysosome holding capacity following fluid-phase uptake in activated cells. We observed a significant reduction in lucifer yellow accumulation in cells co-treated with Torin1 and LPS (Figure 4.7f). Together our data suggest that both AKT and mTOR activity are needed to boost lysosome biogenesis and remodelling upon phagocyte activation. 


\subsection{3 mTOR bolsters immunity by enhancing translation of lysosomal transcripts}

\subsubsection{LPS boosts global protein synthesis in primary macrophages through mTOR}

Our data thus far suggest that LPS activation results in increased lysosome remodelling and hyperactivation of mTOR signalling. We next tested whether LPS stimulates global protein synthesis in primary macrophages using a puromycylation assay, whereby puromycin is incorporated into de novo polypeptides that are can be detected with western blotting. LPS stimulation increased puromycylation incorporation in a time-dependent manner (Figure 4.8), indicating there was an increase in de novo protein levels. Next, we looked at the impact of mTOR inhibition on LPS-mediated general protein synthesis, where Torin1 treatment abrogated the increase in puromycin incorporation. Additionally, using cyclohexamide as a positive control for protein synthesis inhibition, we saw that co-treatment with LPS blocked puromycin incorporation. Altogether the data demonstrate that LPS bolstered global protein synthesis in primary macrophages.

\subsubsection{2 mTOR increases translation of mRNAs encoding lysosomal proteins}

mTOR is a known mediator of global protein synthesis, but can also regulate selective translation of mRNA pools (Masvidal, Hulea, Furic, Topisirovic, \& Larsson, 2017). Thus, we speculated whether mTOR could be selectively increasing the translation of lysosomal mRNAs to coordinate lysosome remodelling following LPS activation. In collaboration with the Topirisovic lab at McGill University, we used polysome profiling to separate mRNA into fractions according to the number of bound ribosomes by sedimentation through a 5-50\% sucrose gradient (Gandin et al., 2014). We isolated the fractionated mRNA and measured the distribution of mRNAs encoding lysosomal proteins by RT-qPCR. For this assay, we used RAW macrophages due to the technical limitations and amount of material required for polysome profiling studies. 
Relative to control resting cells, 2 and 6 hours of LPS stimulation shifted the distribution of mRNAs encoding for LAMP1, ATP6V1H and ATPV1D mRNAs to heavier fractions, indicative of increased translation efficiency (Figure 4.9a-c; Figure A8). We then investigated the impact of mTOR inhibition on lysosomal mRNA distribution. First, Torin1 treatment exerted minimal effect on the distribution of our target mRNAs in control cells (Figure A7). However, Torin 1 treatment did reduce the loading of lysosomal mRNAs in heavy fractions. These findings indicate that LPS induced translation of mRNAs encoding LAMP1, and V-ATPase subunits $\mathrm{H}$ and D via mTOR. Translational regulation of LAMP1 and V-ATPase subunits H and D is further supported by results obtained in primary macrophages, wherein LPS treatment induced LAMP1 and V-ATPase subunit protein levels, without affecting their mRNA levels or protein stability (Figure 4.5a; Figure 4.6c). Interestingly, mRNA distribution of two heavy polysomal housekeeping proteins, $\beta$-actin and PPIA, remained unchanged upon additional of LPS and/or Torin1 (Figure 4.9d, e; Figure A8). It has been reported that both housekeeping proteins are not affected by LPS exposure (Piehler, Grimholt, Øvstebø, \& Berg, 2010). Similarly, the light polysomal housekeeping protein $\beta 2$-microglobulin (B2M) was insensitive to mTOR and LPS (Figure 4.9f, 1; Figure A8). Collectively, our data indicate that translation of mRNAs encoding lysosomal proteins was selectively stimulated during macrophage activation by LPS in an mTORdependent manner.

\subsubsection{4E-BP1 and S6 kinase regulate lysosome expansion in activated phagocytes}

mTORC1 enhances mRNA translation through multiple mechanisms (Roux \& Topisirovic, 2018).

Given that LPS stimulates mTORC1, leading to phosphorylation and activation of S6K and repression of 4E-BPs (Figure 4.7a,b), we next tested whether these translation regulatory factors play a direct role in LPS-induced lysosome expansion. To disrupt S6 kinase function in primary 
macrophages, we used LY258470 (LY2), a potent pharmacological inhibitor of S6 kinase (Figure A6c). First, we showed that LPS-increased puromycylation was abrogated upon S6 kinase inhibition (Figure 4.10a, b). Additionally, we showed that primary macrophages treated with LY258470 abrogated LPS-induced lysosome volume increase (Figure 4.10c, d), but not lysosomal tubulation (Figure 4.10e). This suggests a previously unrecognized uncoupling of lysosome tubulation and expansion. Similarly, inhibition of S6 kinases prevented the LPS-mediated increase in the levels of LAMP1, ATP6V1H and ATP6V1D proteins and other markers (Figure 4.10f, g; Figure A4). LY258470 had no effect on lysosomal transcript levels in resting cells or those coexposed to LPS (Figure A6d). Together, these data suggest that the mTORC1-S6 kinase axis promoted lysosomal protein synthesis to rapidly expand the lysosome size during phagocyte activation.

We next investigated the role of 4E-BPs in regulating lysosome expansion following LPS. For this, we generated RAW macrophages that stably expressed 4E-BP1 ${ }^{4 A l a}$, a phosphorylation deficient mutant of 4E-BP1 carrying alanine substitutions at four phosphorylation sites (Thr37, Thr46, Ser65, and Thr70), rendering it insensitive to mTORC1 regulation (Rong et al., 2008). This form of 4EBP1 constitutively binds to the cap-binding protein eIF4E, preventing the assembly of the eIF4F complex and ribosome to mRNA (Rong et al., 2008). Relative to resting RAW counterparts, LPS augmented total lysosomal volume and lysosome tubulation in RAW cells expressing an empty pBabe retroviral vector (Figure 4.11a-c). In contrast, RAW cells that stably expressed 4E-BP1 $1^{4 A l a}$, failed to boost total lysosome volume in response to LPS, though lysosome tubulation still occurred. The changes in lysosome volume were accompanied by corresponding alterations in lysosomal protein levels. LPS exposure increased lysosomal protein levels (LAMP1, ATP6V1H and ATP6V1D) in RAW cells expressing the empty retroviral vector, compared to 
resting counterparts (Figure 4.11d, e). Whereas, LPS failed to boost the levels of these proteins in RAW cells stably expressing 4E-BP1 ${ }^{4 A l a}$ (Figure 4.11d, e). Collectively, our data revealed that the effects of mTORC1 on lysosome remodelling were facilitated via orchestrated activity of S6Ks and 4E-BPs.

\subsubsection{LPS promotes Ag-presentation and T-cell activation through mTOR and S6K activity}

Our results thus far suggest that LPS induces rapid lysosomal expansion in primary phagocytes via a mTOR-dependent boost in translation of lysosomal transcripts. Next, we sought to investigate the functional implication of LPS-mediated escalation in translation and lysosomal volume following activation. We postulated that LPS-mediated lysosome expansion and increase in holding capacity may enhance antigen presentation by BMDCs. To test this, we used BMDCs from $\mathrm{C} 57 \mathrm{Bl} / 6 \mathrm{~J}$ and $\mathrm{C} 3 \mathrm{H} / \mathrm{Hen}$ mice that respectively carry MHC-II haplotypes I-A $\mathrm{A}_{\mathrm{b}}$ and $\mathrm{I}-\mathrm{A}_{\mathrm{k}}$. I- $\mathrm{A}_{\mathrm{b}}$ and $\mathrm{I}-\mathrm{A}_{\mathrm{k}}$ expressing BMDCs were then respectively fed antigenic peptides, Ea52-68 and Hen Egg Lysozyme (HEL), for 4 and 6 hours in the presence or absence of LPS. We used Aw3.18.14 monoclonal staining of fixed but unpermeabilized BMDCs to detect surface delivery of I-A $A_{k}$ : HEL48-62 complexes (Dadaglio, Nelson, Deck, Petzold, \& Unanue, 1997). However, we were unable to detect intracellular complexes, as this antibody could not detect MHC-II:peptide complex after permeabilization (data not shown). On the other hand, we could detect total I$A_{b}$ :Ea52-68 (internal and surface level) complex formation by staining fixed and saponinpermeabilized BMDCs with the monoclonal Y-Ae antibody (Murphy et al., 1992). Importantly, treatment with LPS stimulated the formation and/or delivery of the MHC-II:peptide complexes even at $4 \mathrm{~h}$ and more potently at $6 \mathrm{~h}$ (Figure 4.12a-c; Figure A9a,b). When cells were not given 
antigens, signal was reduced to background, demonstrating that the fluorescence signal was dependent on MHC-II:antigen complex formation (Figure 4.12a-c; Figure A9a,b).

We then inquired if antigen presentation was dependent on mTOR and S6K activities by co-treating cells with Torin1 and LY258470, respectively. Remarkably, these inhibitors reduced antigen presentation of both Ea52-68 and HEL48-62 in unstimulated and LPS-treated cells (Figure 4.12a-c; Figure A9a,b). These data indicate that enhanced translation controlled by the mTORC1S6K axis is necessary for efficient antigen presentation by BMDCs.

Next, we were interested in investigating whether pre-activated BMDCs whose lysosomal population is remodeled are able to strengthen T-cell priming and activation. To test this, we used the I-A ${ }^{b}$ restricted E $\alpha$ - specific $1 \mathrm{H} 3.1 \mathrm{~T}$ cell hybridoma cell-line for activation assays with preactivated dendritic cells expressing I-A ${ }^{\mathrm{b}}: \mathrm{E} \alpha_{52-68}$ complexes. Briefly, we loaded BMDCs with E $\alpha_{52-}$ 68 in the presence and/or absence of LPS and inhibitors and then treated them with a mild fixative. We co-incubated pre-activated BMDCs with T-cells at a 1:2 ratio for 40 hours and then measured T-cell dependent secretion of IL-2 to quantify T-cell activation. We saw that acute stimulation of BMDCs for 4 and 6 hours was sufficient to significantly increase T-cell dependent IL-2 secretion (Figure 4.12d). Moreover, when we blocked mTOR and S6 kinase activities in BMDCs, T-cell dependent IL-2 secretion was blunted, supporting their role in BMDC remodelling. Collectively with all our observations, we propose that this increase in immune response is in part due to rapid lysosomal expansion and remodelling. In section 6.2, I present an overall model, discuss possible mechanisms controlling lysosome remodelling, implications in cellular adaptation and suggest future directions to identify and further our understanding of the possible regulatory machinery. 
$4.3 \quad$ Figures 
Figure 4.1
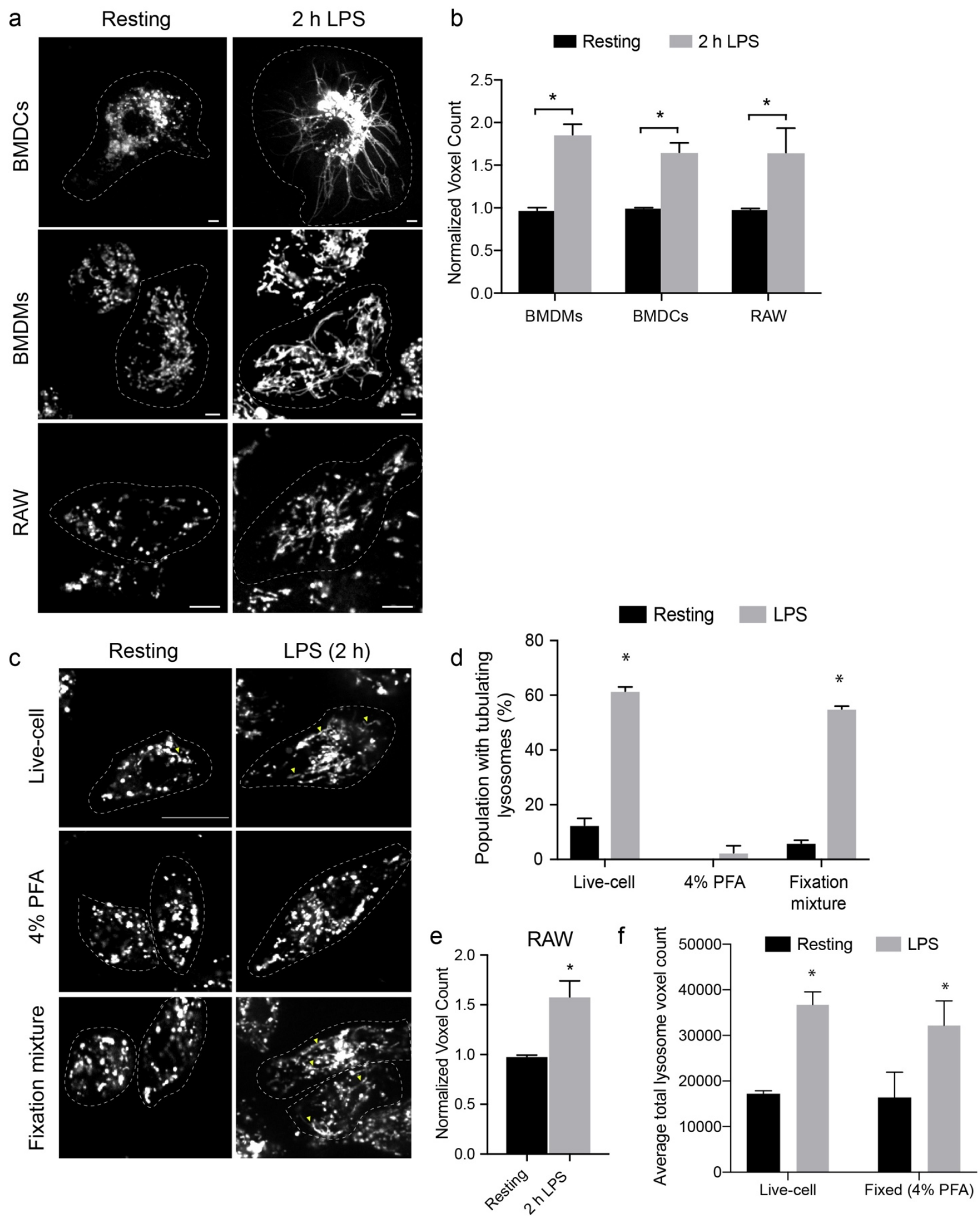
Figure 4.1: LPS-activation augments lysosome volume in phagocytes.

(a) Lysosomes in bone-marrow derived macrophages (BMDMs), bone-marrow derived dendritic cells (BMDCs), and in RAW macrophages before and after $2 \mathrm{~h}$ of LPS stimulation, the latter causing extensive lysosome tubulation. Images were acquired by live-cell spinning disc confocal microscopy. Scale bar $=5 \mu \mathrm{m}$. (b) Relative lysosome volume between counterpart resting and LPS-treated phagocytes acquired by live-cell spinning disc confocal imaging. (c) RAW macrophage lysosomes labeled with fluid phase probes fluorescent probes, were imaged live or fixed with 4\% PFA or mixture of PFA and glutaraldehyde as explained in methods. (d) Percent lysosome tubulation was recorded within the population for cells exhibiting 4 or more lysosomal tubules longer then $4 \mu \mathrm{m}$ (d) Relative lysosome volume in resting and LPS-treated RAW macrophages using fixation method to preserve lysosome tubules. (f) Lysosomal voxel comparison for cells fixed with 4\% PFA, which causes tubules to collapse into punctate lysosomes. All experiments were repeated at least three independent times. Data are based on 30-40 cells per condition per experiment and are shown as the mean \pm SEM. Statistical analysis was done using one-way ANOVA and unpaired Student's t-test, where the asterisk * indicates a significant increase in lysosome volume relative to resting phagocytes $(\mathrm{p}<0.05)$. 
a
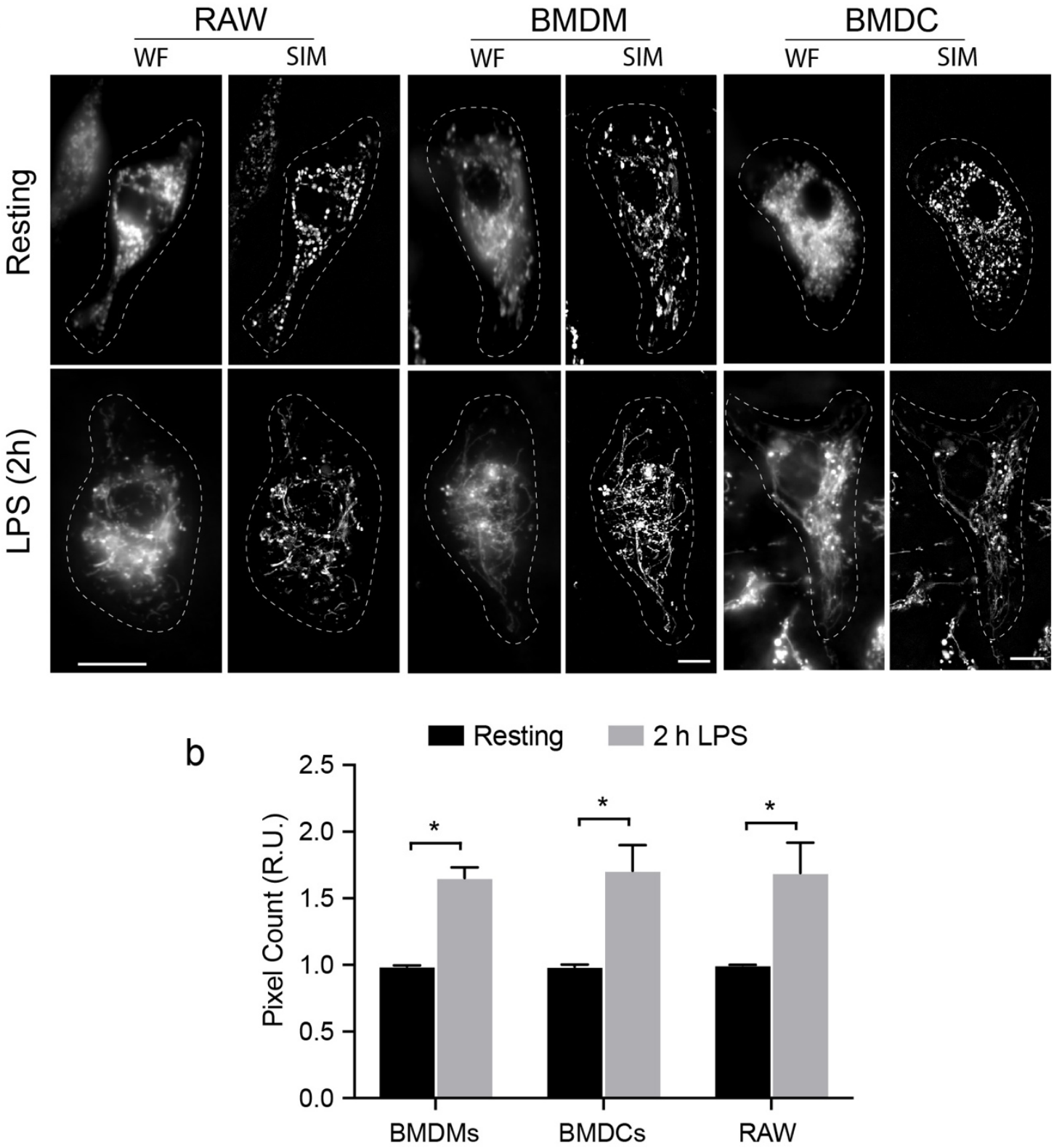

Figure 4.2: Super-resolution microscopy showing expanded lysosome network.

(a) Wide field (WF) illumination or structured illumination microscopy (SIM) images of lysosomes in a RAW macrophage, bone marrow derived macrophage (BMDM) and bone-marrow derived dendritic cell (BMDC), before and after $2 \mathrm{~h}$ of LPS stimulation. Scale bar $=5 \mu \mathrm{m}$. (b) Relative lysosome area from the mid-section of resting and LPS-activated phagocytes using images acquired by SIM-enacted super- resolution microscopy. Experiments were repeated three independent times. Data are based on 30-40 cells per condition per experiment and are shown as the mean \pm SEM. 
a
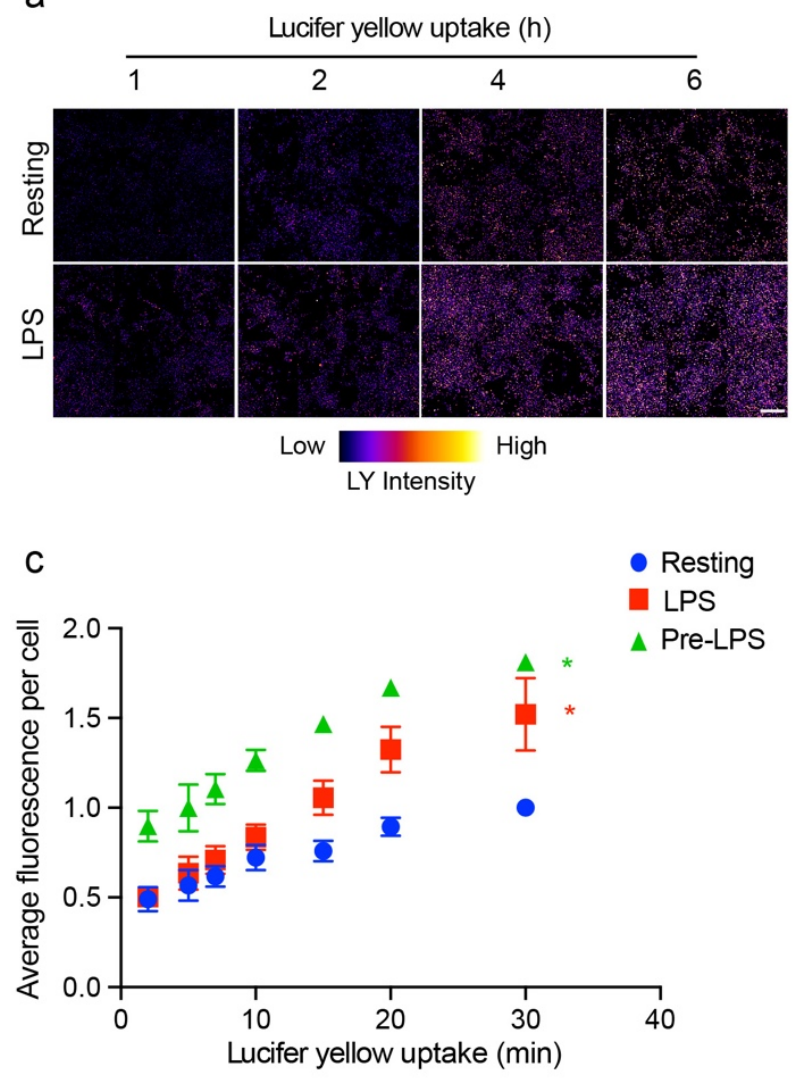

b

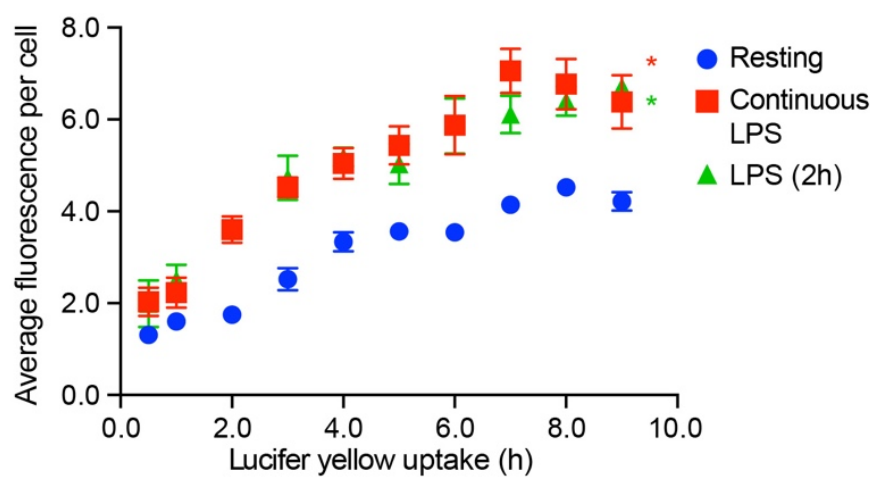

d

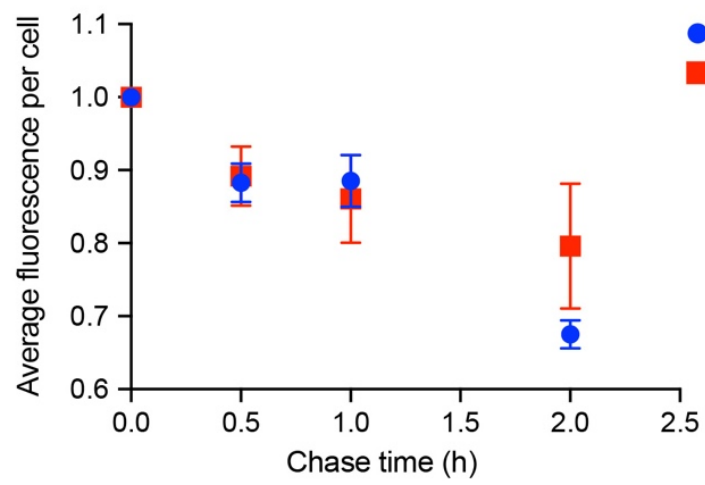

Figure 4.3: Macrophage activation increases lysosomal holding capacity.

(a) Image compilation of 6 representative fields in false-colour showing changes in intensity of Lucifer yellow acquired by endocytosis over the indicated time in resting primary macrophages or macrophages stimulated with LPS. Scale $=250 \mu \mathrm{m}$. Colour scale: $0-4095$ (low-high). (b) Accumulation of Lucifer yellow continuously endocytosed over indicated timeframe in resting cells, cells activated with LPS for $2 \mathrm{~h}$ or co-activated with LPS continuously. (c) Rate of pinocytosis of Lucifer yellow in primary macrophages treated as indicated. (d) Retention of Lucifer yellow in resting or LPS-treated primary macrophages after $0.5 \mathrm{~h}$ internalization and chase in probe-free medium over indicate times. In all cases, fluorescence measurements were acquired by confocal high-content fluorometer plate-imager. Data are shown as the mean \pm SEM from at least 3 independent experiments. Statistical analysis was done using an Analysis of Covariance, whereby controlling for time as a continuous variable. An asterisk indicates a significant increase in Lucifer yellow for that series relative to resting phagocytes $(\mathrm{p}<0.05)$. 
Figure 4.4

a

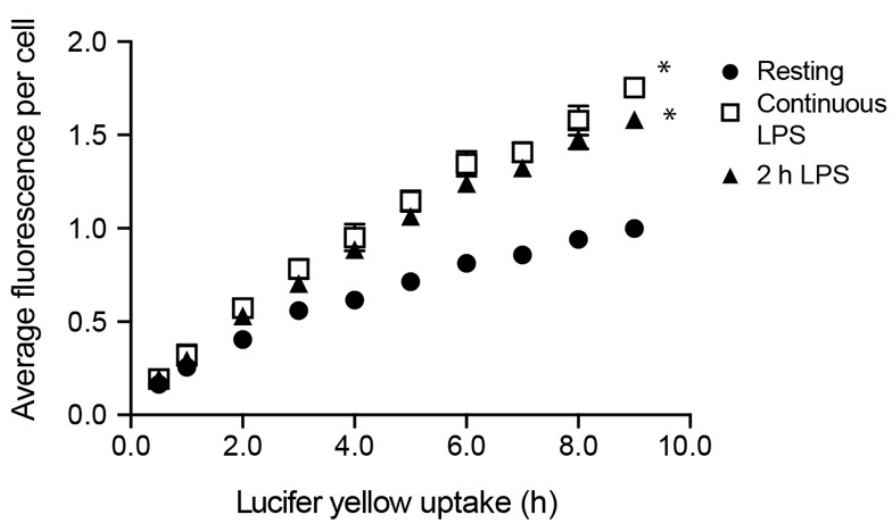

b
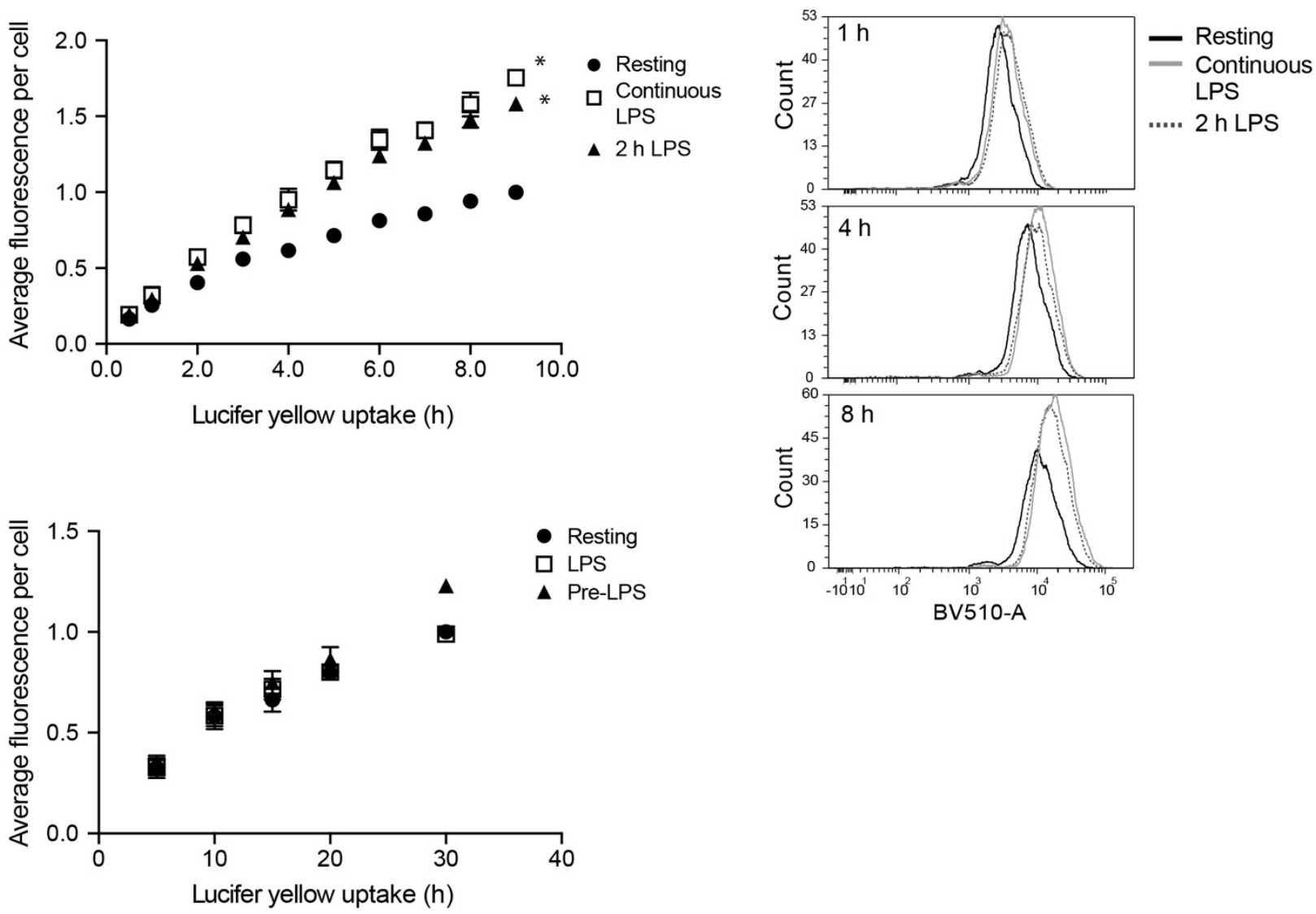

C

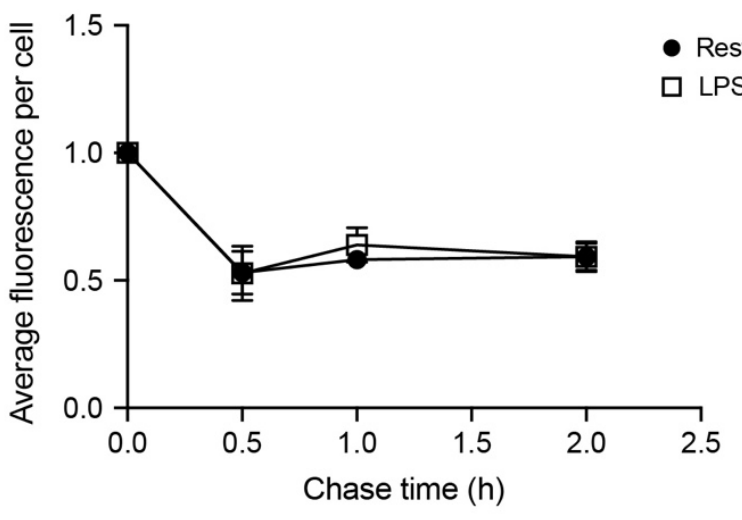

d
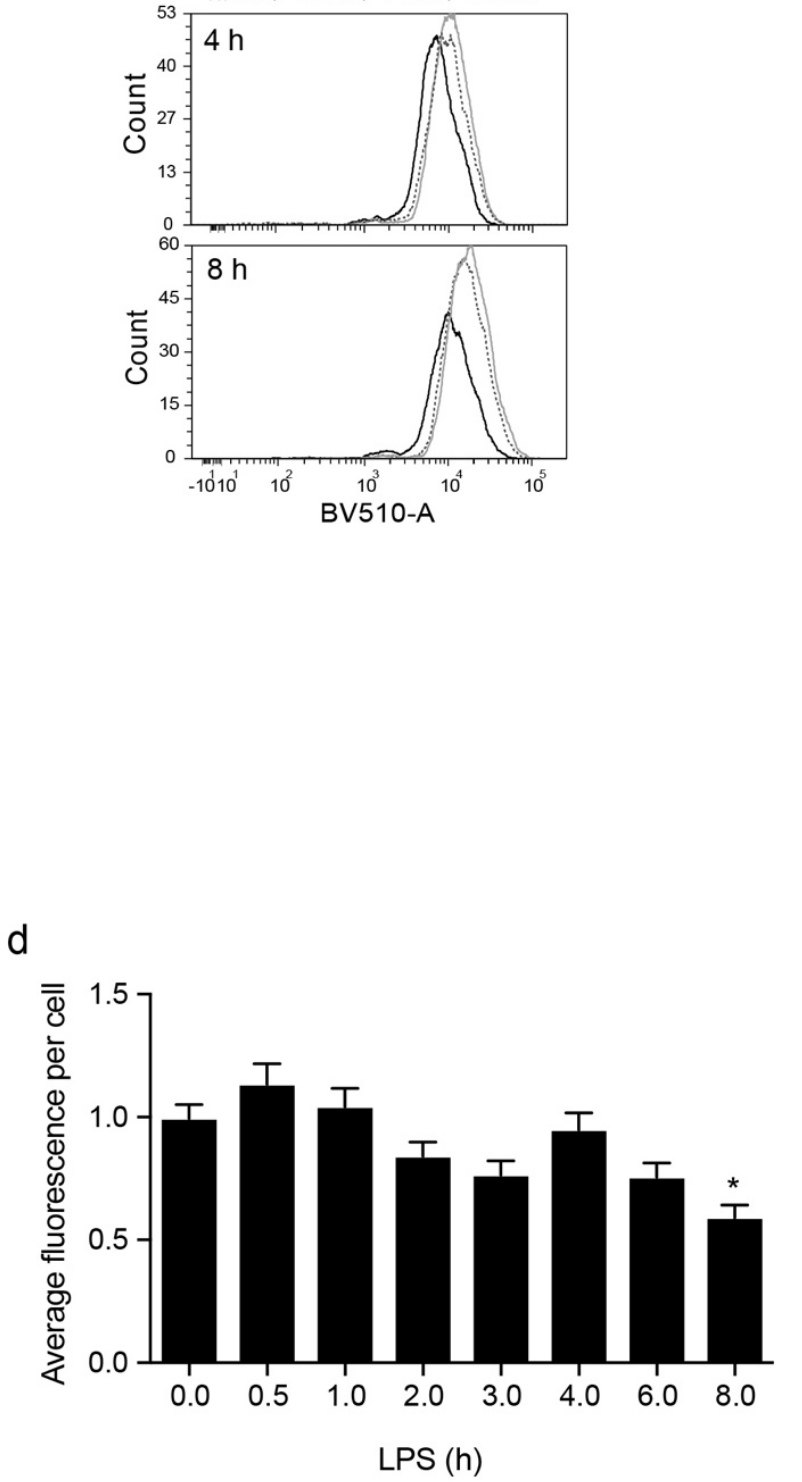
Figure 4.4: Activated RAW macrophages have a larger lysosome holding capacity.

(a) Accumulation of Lucifer yellow (LY) in resting and activated RAW macrophages. RAW cells were stimulated and then allowed to internalize LY over time. (b) Pinocytosis rate by quantifying uptake of Lucifer yellow in RAW macrophages treated as indicated. (c) Retention of Lucifer yellow chased in probe-free medium in RAW cells previously treated as indicated and pre-labelled with LY for $1 \mathrm{~h}$. In all cases, fluorescence measurements were done by flow cytometry. (d) Pinocytosis in increasingly maturing DCs exposed to LPS. Microscopy was used to measure the uptake of fluorescent dextran for $30 \mathrm{~min}$ by DCs exposed to LPS over indicated time points. Shown is the mean \pm SEM from at least 3 experiments. For statistical analysis, ANOVA or Analysis of Covariance was used, whereby an asterisk indicates a significant difference in fluorescent probe levels compared to resting $\left({ }^{*} \mathrm{p}<0.05\right)$. 
Figure 4.5

a
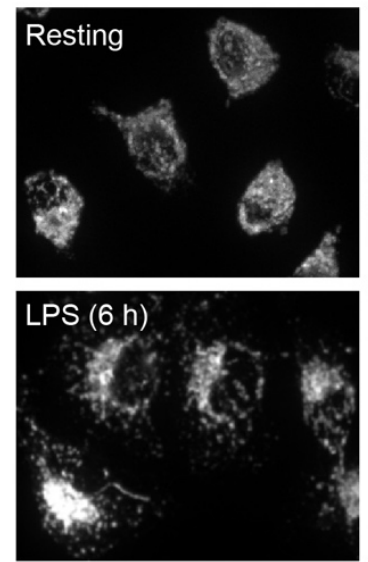

C
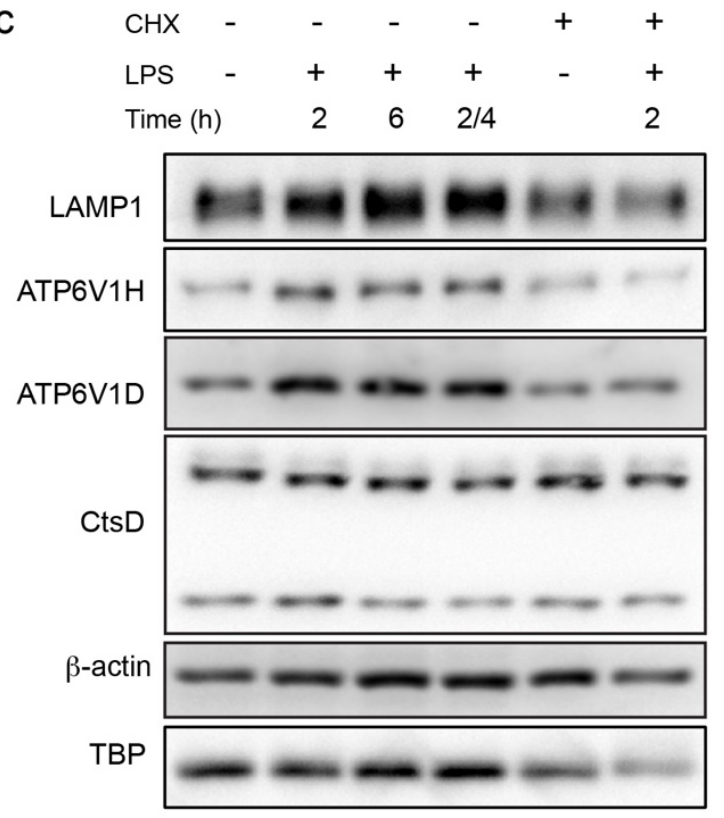

e
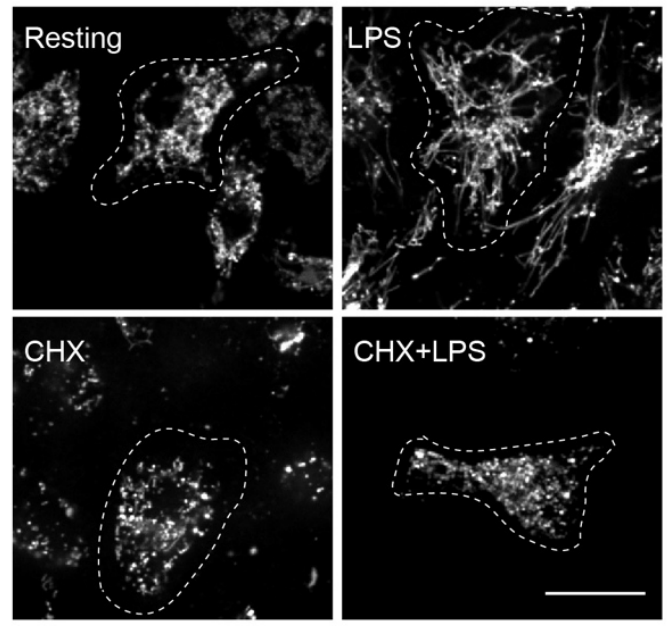

b

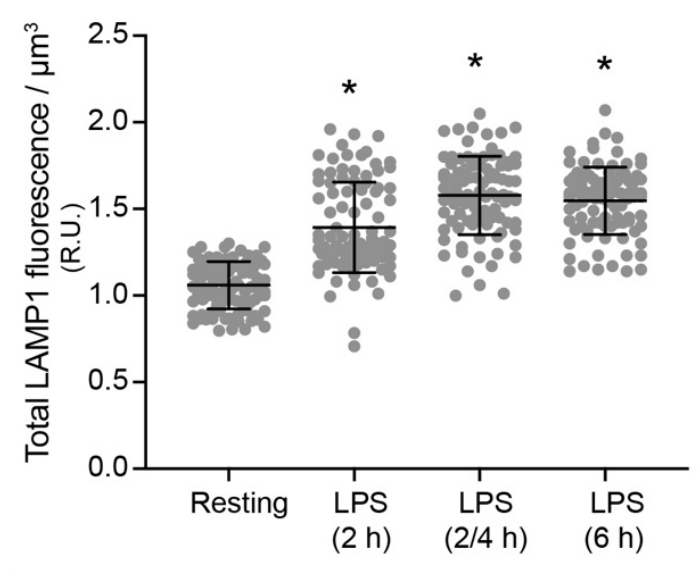

d

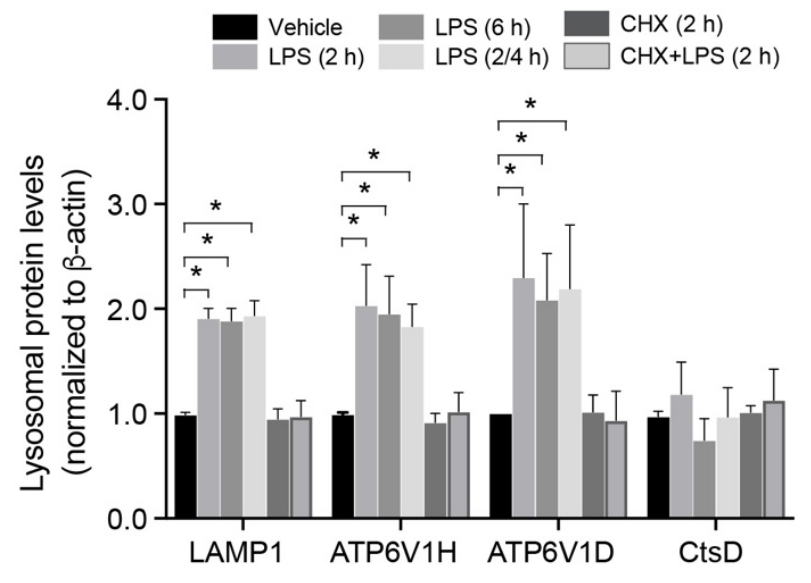

f

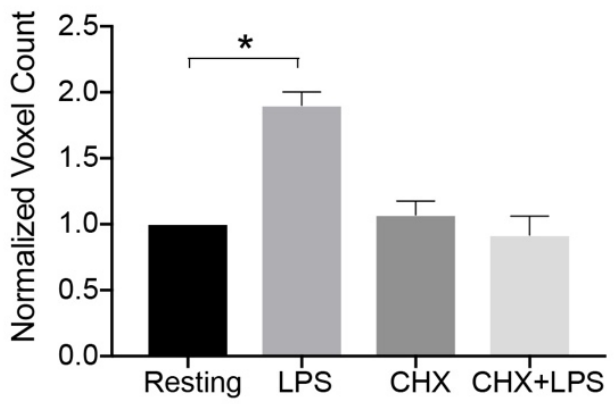


Figure 4.5: Lysosome remodelling requires protein biosynthesis.

(a) Endogenous LAMP1 structures in resting and LPS-activated BMDMs. (b) Quantification of total LAMP1 levels in BMDMS per micron ${ }^{3}$. (c) Western blot analysis of whole cell lysates from resting primary macrophages or macrophages exposed to the indicated combinations and time of LPS and cycloheximide (CHX). (d) Quantification of Western blots showing the levels of LAMP1, cathepsin $\mathrm{D}(\mathrm{CtsD})$ and the V-ATPase $\mathrm{V}_{1}$ subunit $\mathrm{H}$ and $\mathrm{D}$ normalized to actin. Data shown as the mean \pm standard error of the mean from at least 3 independent experiments. For $A$ and $B$, " $2 / 4$ " indicates cells stimulated with $2 \mathrm{~h}$ of LPS, followed by a $4 \mathrm{~h}$ chase, whereas 2 and $6 \mathrm{~h}$ represent cells continuously exposed to LPS. (e) Live-cell spinning disc confocal micrographs of prelabelled lysosomes in resting primary macrophages or those stimulated with LPS and/or cycloheximide. Scale bar $=5 \mu \mathrm{m}$. (f) Relative lysosome volume between resting primary macrophages and those exposed to specified conditions. Shown is the mean \pm SEM mean from 30-40 cells for each condition and experiment, across at least 3 independent experiments. Statistical analysis was done with ANOVA and unpaired Student's t-test. The asterisk * indicates a significant difference $(\mathrm{p}<0.05)$. 
Figure 4.6
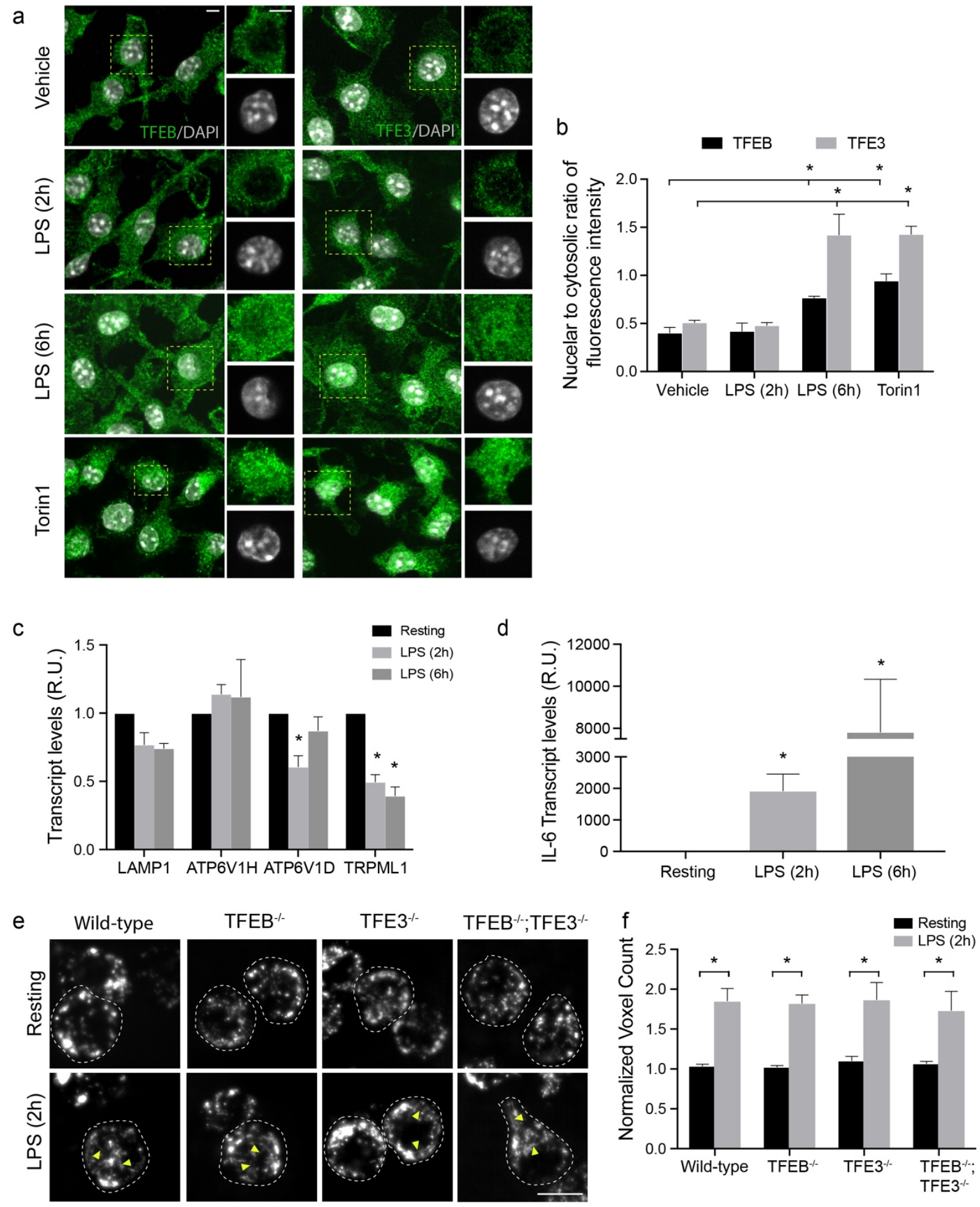
Figure 4.6: Lysosome remodelling is independent of TFEB and TFE3 activation.

(a) TFEB and TFE3 subcellular localization in resting primary macrophages (vehicle) or those treated with LPS for 2 or $6 \mathrm{~h}$, or with torin 1 . Green $=$ TFEB or TFE3 immunofluorescence signal; white $=$ nuclei stained with DAPI. Areas within dashed boxes are magnified as insets. (b) Nuclear to cytosolic ratio of TFEB or TFE3 fluorescence intensity. Shown is the mean \pm SEM from 30-40 cells per condition per experiment across at least 3 independent experiments. (c,d) Relative mRNA levels of select lysosomal genes (c) or interleukin-6 (d) in activated primary macrophages relative to Abt1 housekeeping gene and normalized against resting cells. Quantification was done with qRT-PCR by measuring the $\Delta \Delta \mathrm{Ct}$ as described in methods. Shown is the mean \pm standard error of the mean from four independent experiments. (e) Lysosomes in wild-type, $t f e b^{-/}$, $t f e 3^{-/-}$ and $t f e b^{-/} t f e 3^{-/}$RAW strains before and after $2 \mathrm{~h}$ of LPS stimulation. Images were acquired by live-cell spinning disc confocal microscopy. Yellow arrowheads illustrate tubular lysosomes. (f) Relative lysosome volume between LPS-treated and resting counterpart RAW strains acquired by live-cell spinning disc confocal imaging. Shown is the mean \pm SEM from 30-40 cells per condition per experiment across three independent experiments. All statistical analysis was done with ANOVA and unpaired Student's t-test. The asterisk * indicates a significant difference relative to resting condition $(\mathrm{p}<0.05)$. For a and $\mathrm{e}$, scale bar $=5 \mu \mathrm{m}$. 
Figure 4.7

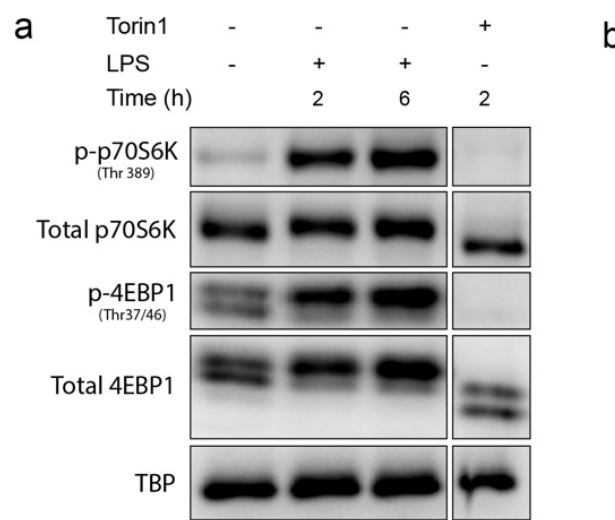

c

$$
\text { c }
$$
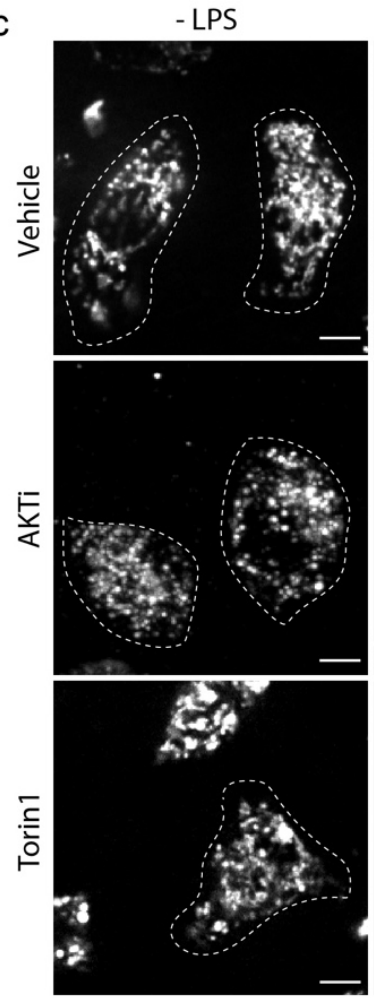

d

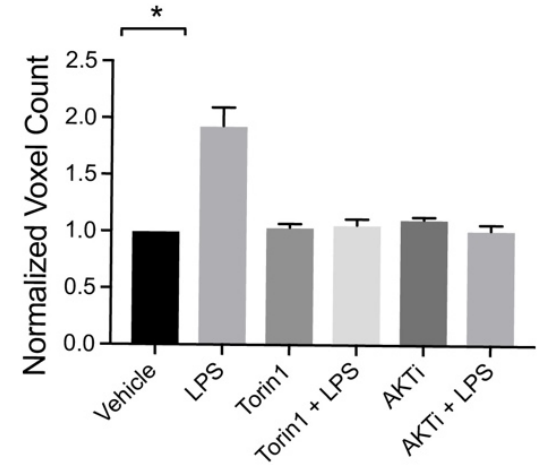

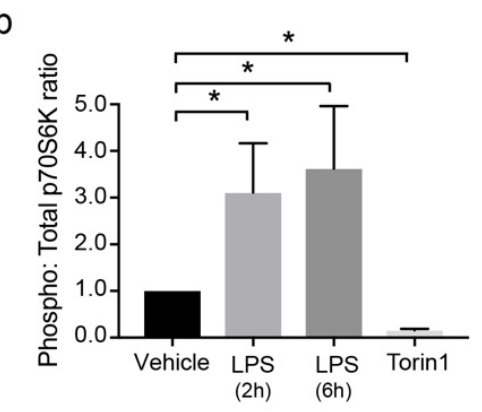

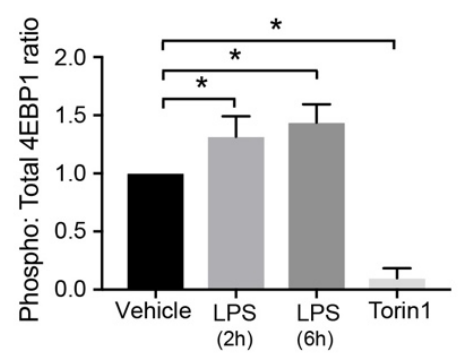

e
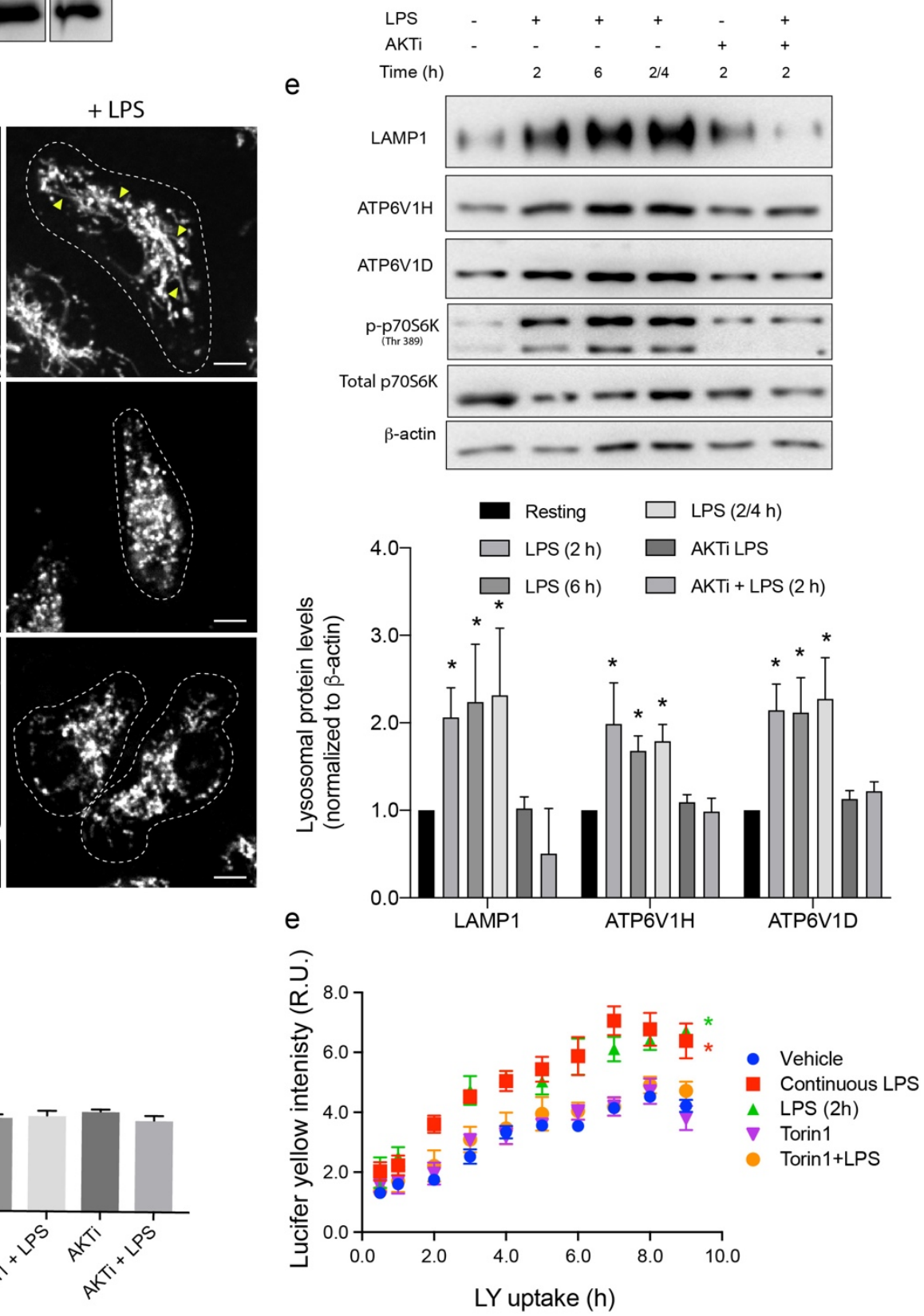
Figure 4.7: mTOR stimulates lysosome volume and holding capacity.

(a) Western blot analysis of whole cell lysates from resting and activated primary macrophages. Total levels and phosphorylation status of S6K and 4EBP1 were monitored using indicated antibodies. TBP served as a loading control. (b) Normalized ratio of p-p70S6K and p-4EBP1 to total $\mathrm{p} 70 \mathrm{~S} 6 \mathrm{~K}$ and $4 \mathrm{E}-\mathrm{BP} 1$ protein. Shown is the mean \pm standard deviation from three independent blots. (c) Lysosomes in primary macrophages were pre-treated with a vehicle (DMSO), Akti or torin1, followed by $2 \mathrm{~h}$ LPS stimulation where indicated. Images were acquired by live-cell spinning disc confocal microscopy. Scale bar $=5 \mu \mathrm{m}$. (d) Lysosome volume in primary macrophages treated as indicated normalized to resting macrophages. Shown is the mean \pm standard error of the mean from 30-40 cells per condition per experiment across three independent experiments. (e) Quantification of pinocytic capacity in macrophages treated as indicated. Shown is the mean \pm SEM from four independent experiments. For $b$ and d, data was statistically analysed with ANOVA and unpaired Student's t-test $(* \mathrm{p}<0.05)$. For E, data were statistically assessed using Analysis of Covariance, whereby controlling for time as a continuous variable. An asterisk indicates a significant increase in Lucifer yellow for that series relative to resting phagocytes $\left({ }^{*} \mathrm{p}<0.05\right)$. 


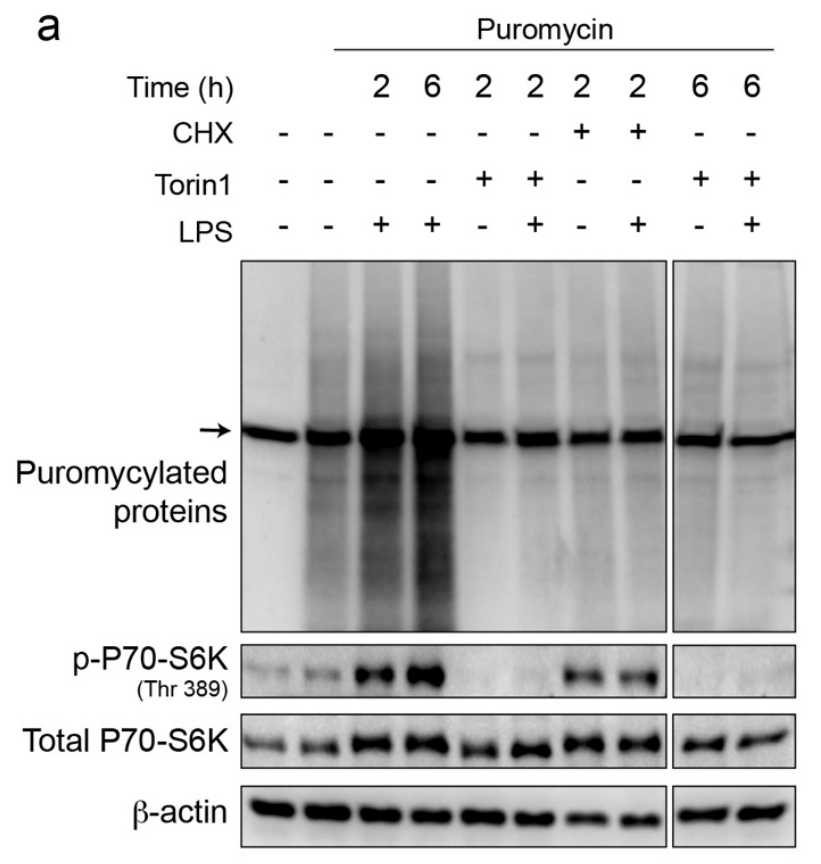

b

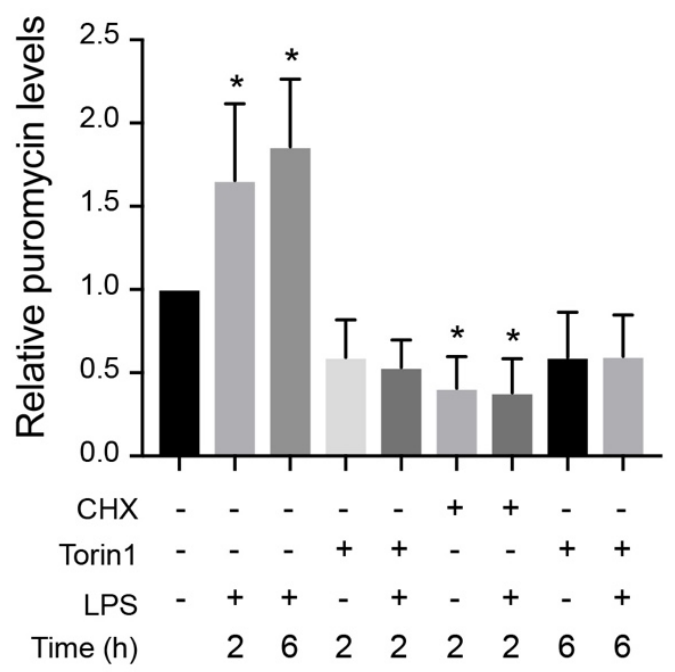

Figure 4.8: LPS stimulates global protein synthesis in primary macrophages.

(a) Western blot analysis of protein puromycylation in resting and activated primary macrophages. LPS increases the amount of puromycylation indicating a boost in global protein synthesis that is blocked by mTOR inhibitors or cycloheximide. Lane 1 are control lysates from cells not exposed to puromycin. The band indicated by arrow is a non-specific band recognized by the antipuromycin antibody. p-p70S6K and $\beta$-actin were used to monitor mTOR status and as a loading control, respectively. (b) Normalized puromycylation signal (excluding non-specific band, indicated by arrow) normalized over $\beta$-actin signal. Data is shown as the mean \pm SD from four independent experiments. Statistical analysis was done with an ANOVA, where * indicates conditions that are statistically distinct from control $(* \mathrm{p}<0.05)$. 
Figure 4.9

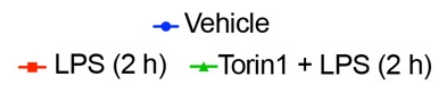$$
+ \text { LPS }(6 h) \stackrel{- \text { Vehicle }}{\rightarrow-\text { Torin1 + LPS }(6 h)}
$$

a

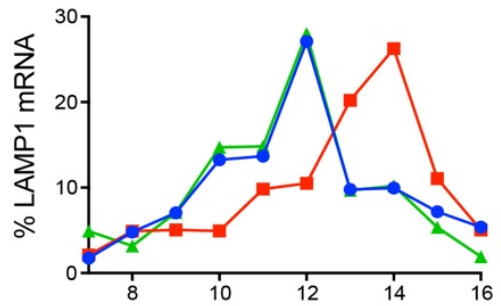

b

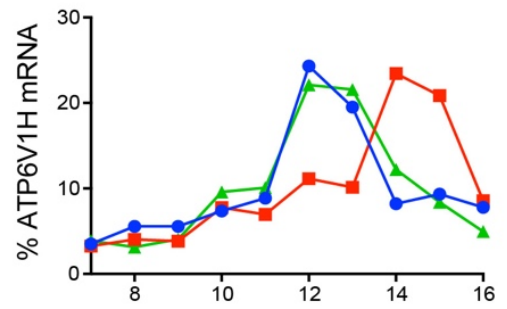

C

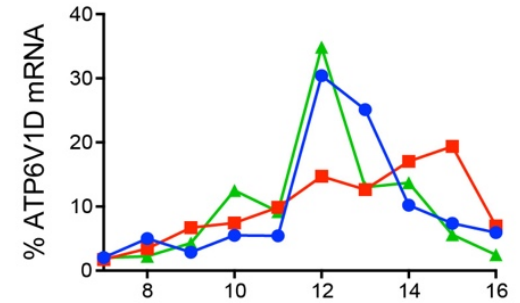

d

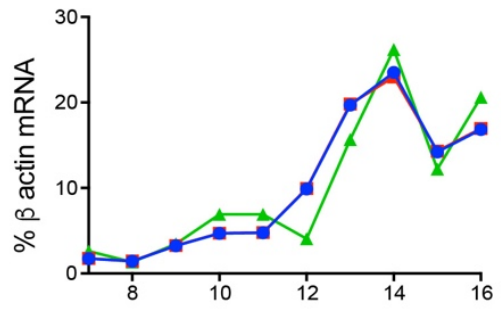

e

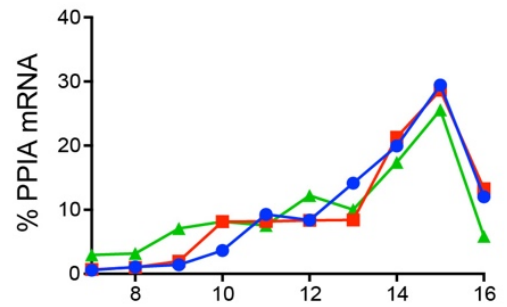

f

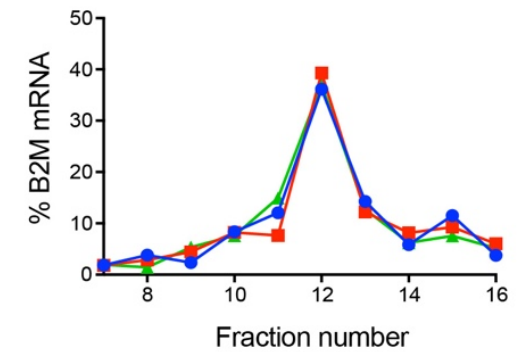

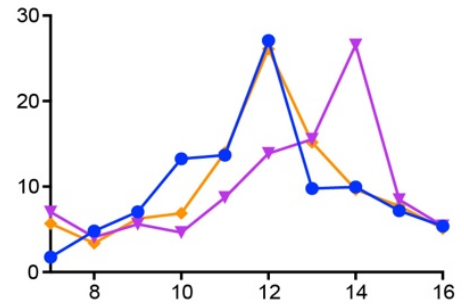
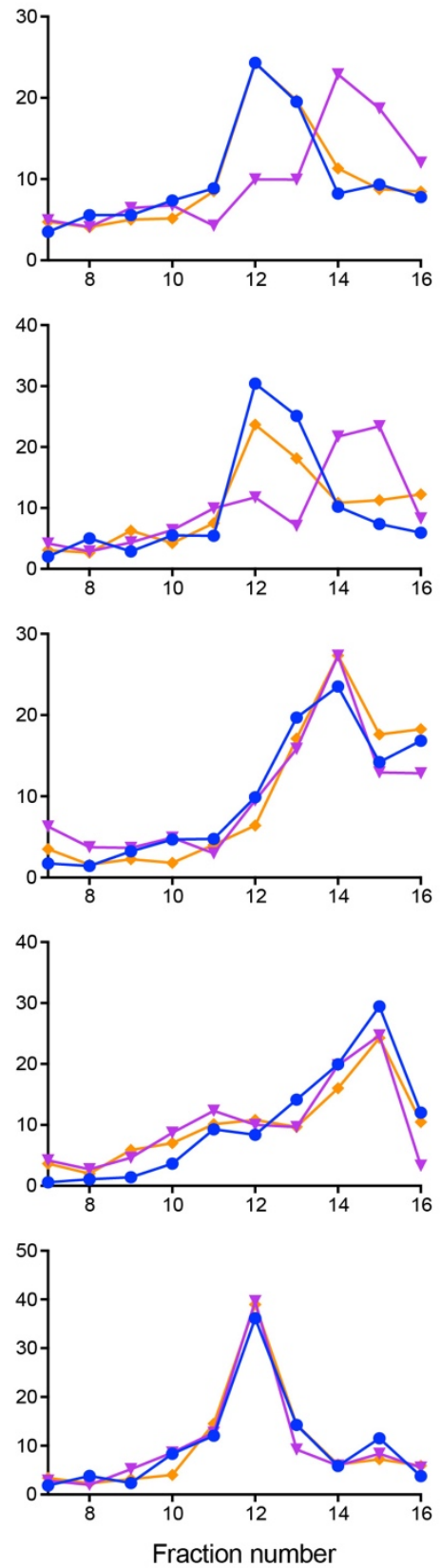
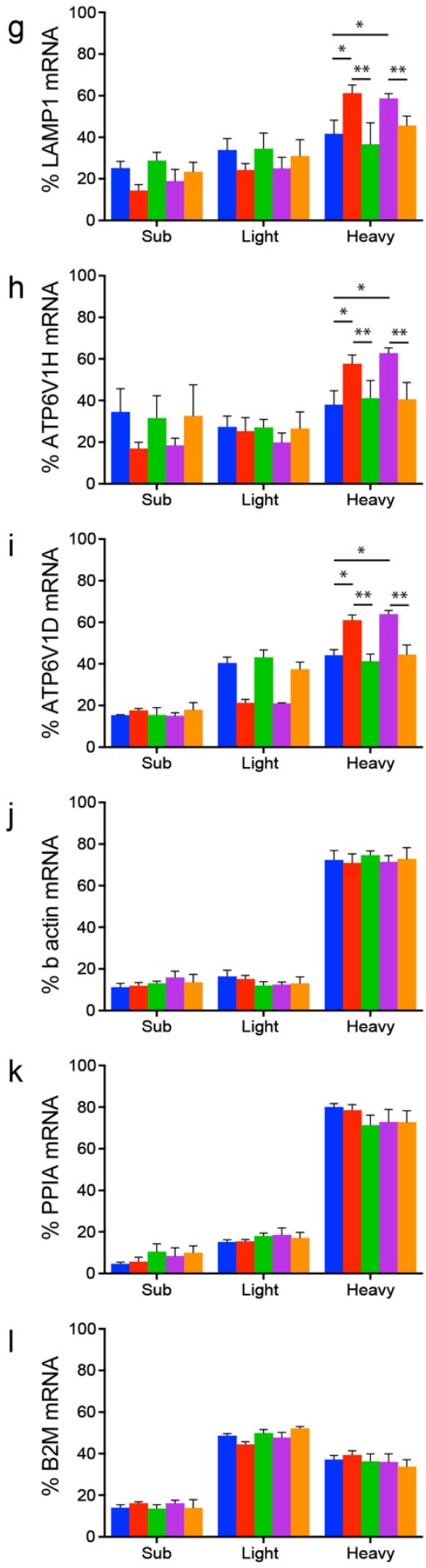
Figure 4.9: LPS increases translation of mRNAs encoding lysosomal proteins in an mTORdependent manner.

(a-f) Percent of target mRNA (a: LAMP1, b: ATP6V1H, c: ATP6V1D, d: $\beta$-actin, e: PPIA, and f: B2M) associated with each ribosome fraction in resting, LPS- or LPS/ Torin1-treated RAW cells. Left and middle panels show $2 \mathrm{~h}$ and $6 \mathrm{~h}$ treatments, respectively. Shown is a representative experiment (as the mean percentage \pm SEM) from four independent experiments, each of which contained three technical replicates. (g-l) Right panels: Pooled percent mRNA in subpolysomal (fractions 7-10), light polysome (fractions 11 and 12) and heavy polysomes (fractions 13-16). Shown is the mean percent \pm SD from four independent experiments with each point in triplicate for each experiment and mRNA. Heavy fractions were statistically analysed by ANOVA and Tukey's post-hoc test, where * indicates statistical difference from resting conditions, while ** indicates differences between LPS and LPS + Torin 1 conditions within 2 and $6 \mathrm{~h}$ exposure. 
Figure 4.10
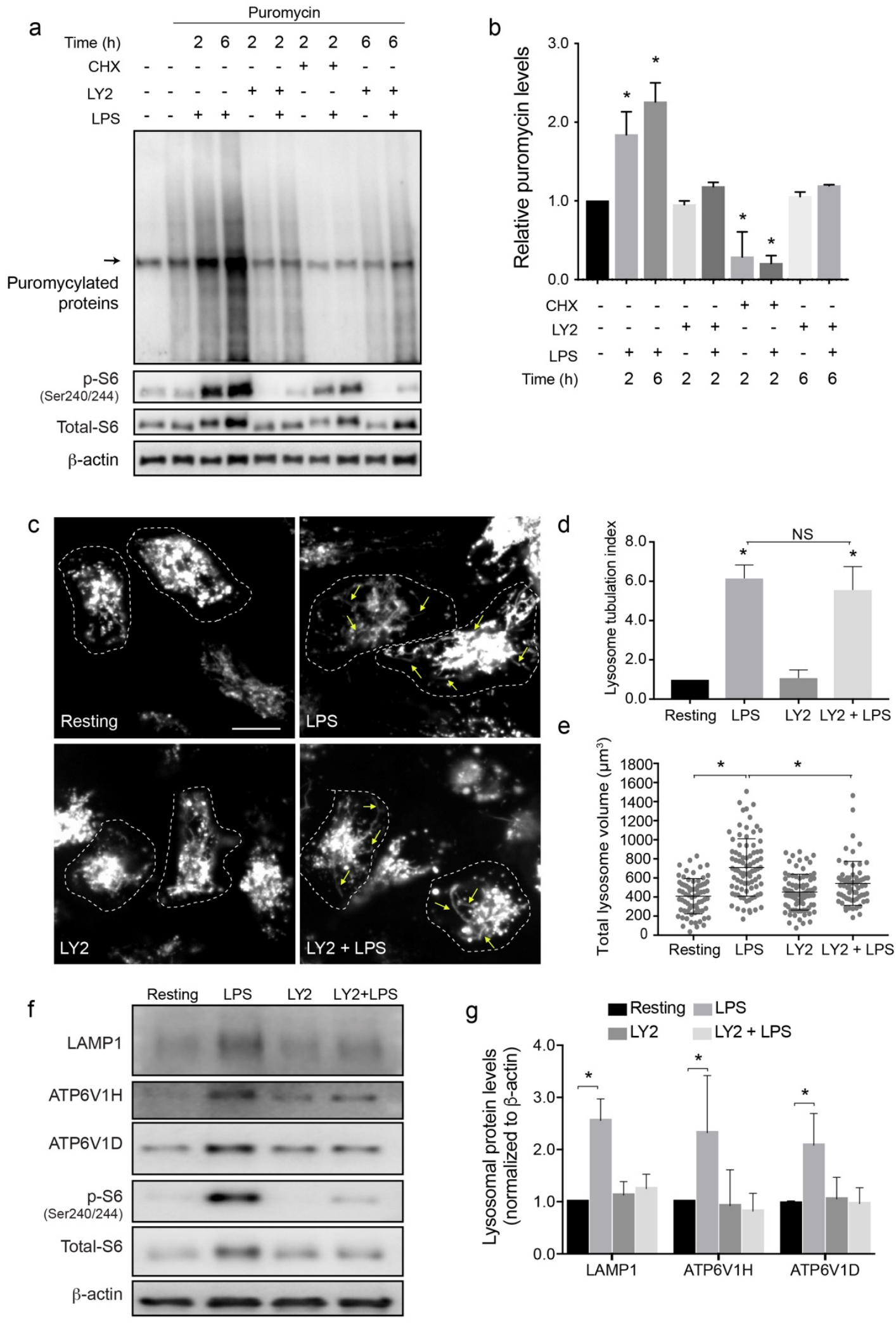
Figure 4.10: S6 kinase is required for the LPS-mediated lysosome expansion.

(a) Western blot analysis of protein puromycylation in resting and activated primary macrophages. LPS increases the amount of puromycylation indicating a boost in global protein synthesis that is blocked by S6k inhibitor or cycloheximide. Lane 1 are control lysates from cells not exposed to puromycin. The band indicated by arrow is a non-specific band recognized by the anti-puromycin antibody. p-S6 and $\beta$-actin were used to monitor S6K status and as a loading control, respectively. (b) Normalized puromycylation signal (excluding non-specific band, indicated by arrow) normalized over $\beta$-actin signal. (c) Lysosomes in primary macrophages were pre-treated with LY258470 (LY2) followed by $2 \mathrm{~h}$ LPS stimulation where indicated. Images were acquired by livecell spinning disc confocal microscopy. Scale bar $=5 \mu \mathrm{m}$. (d) Lysosomal tubulation was scored for each condition as shown, where a tubule was defined as longer than $4 \mu \mathrm{m}$ in length. Tubulation index was determined by normalizing scores to resting cells. (e) Total lysosome volume in primary macrophages treated as indicated. For $\mathbf{b}$ and $\mathbf{c}$, shown are the mean \pm SEM from 30-40 cells per condition per experiment, across three independent experiments. (f) Western blot analysis of whole cell lysates from resting and activated primary macrophages with or without LY258470. (g) Quantification of Western blots showing the levels of LAMP1 and the V-ATPase $\mathrm{V}_{1}$ subunits $\mathrm{H}$ and $\mathrm{D}$, normalized to $\beta$-actin. pS6K and total S6K blots are shown to support the effectiveness of LY258470 treatment. Shown is the mean \pm SD of the mean from five independent blots. For $\mathbf{b}, \mathbf{c}$ and $\mathbf{e}$, data were statistically analysed with ANOVA and unpaired Student's t-test $\left({ }^{*} \mathrm{p}<0.05\right)$. 
Figure 4.11
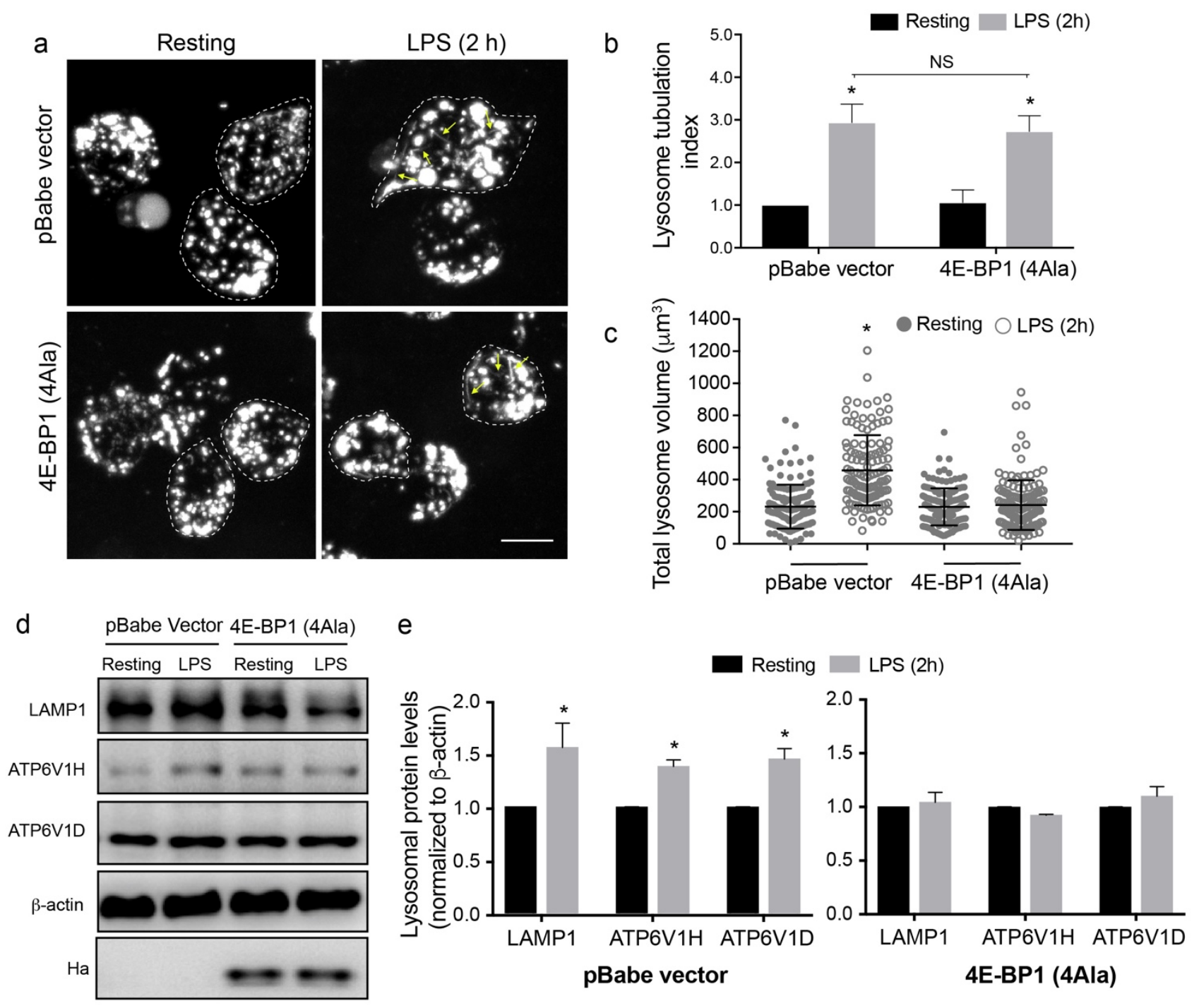
Figure 4.11: Active 4E-BP1 suppresses LPS-mediated lysosome expansion.

(a) Lysosomes in resting or LPS stimulated ( $2 \mathrm{~h}$ ) RAW cells stably expressing the 4E-BP1 (4Ala) phosphorylation mutant or empty pBabe vector. Images were acquired by live-cell spinning disc confocal microscopy. Scale bar $=10 \mu \mathrm{m}$. (b) Lysosomal tubulation was scored for each, where a tubule was defined as longer than $4 \mu \mathrm{m}$ in length. Tubulation index was determined by normalizing scores to resting. (c) Total lysosome volume in engineered RAW macrophages treated as indicated. For $\mathbf{b}$ and $\mathbf{c}$, shown are the mean \pm SEM from 30-40 cells per condition per experiment, across three independent experiments. (d) Western blot (WB) analysis of whole cell lysates from stable cell lines. (e) Quantification of blots showing the levels of LAMP1 and the V-ATPase V 1 subunits $\mathrm{H}$ and $\mathrm{D}$, normalized to $\beta$-actin for both cell lines. Anti-HA blot demonstrates expression of 4EBP $1^{4 A l a}$. Shown is the mean $\pm \mathrm{SD}$ from 3 independent blots. For $\mathbf{b}, \mathbf{c}$ and $\mathbf{e}$, data were statistically analysed with ANOVA and unpaired Student's t-test $\left({ }^{*} \mathrm{p}<0.05\right)$. 
Figure 4.12

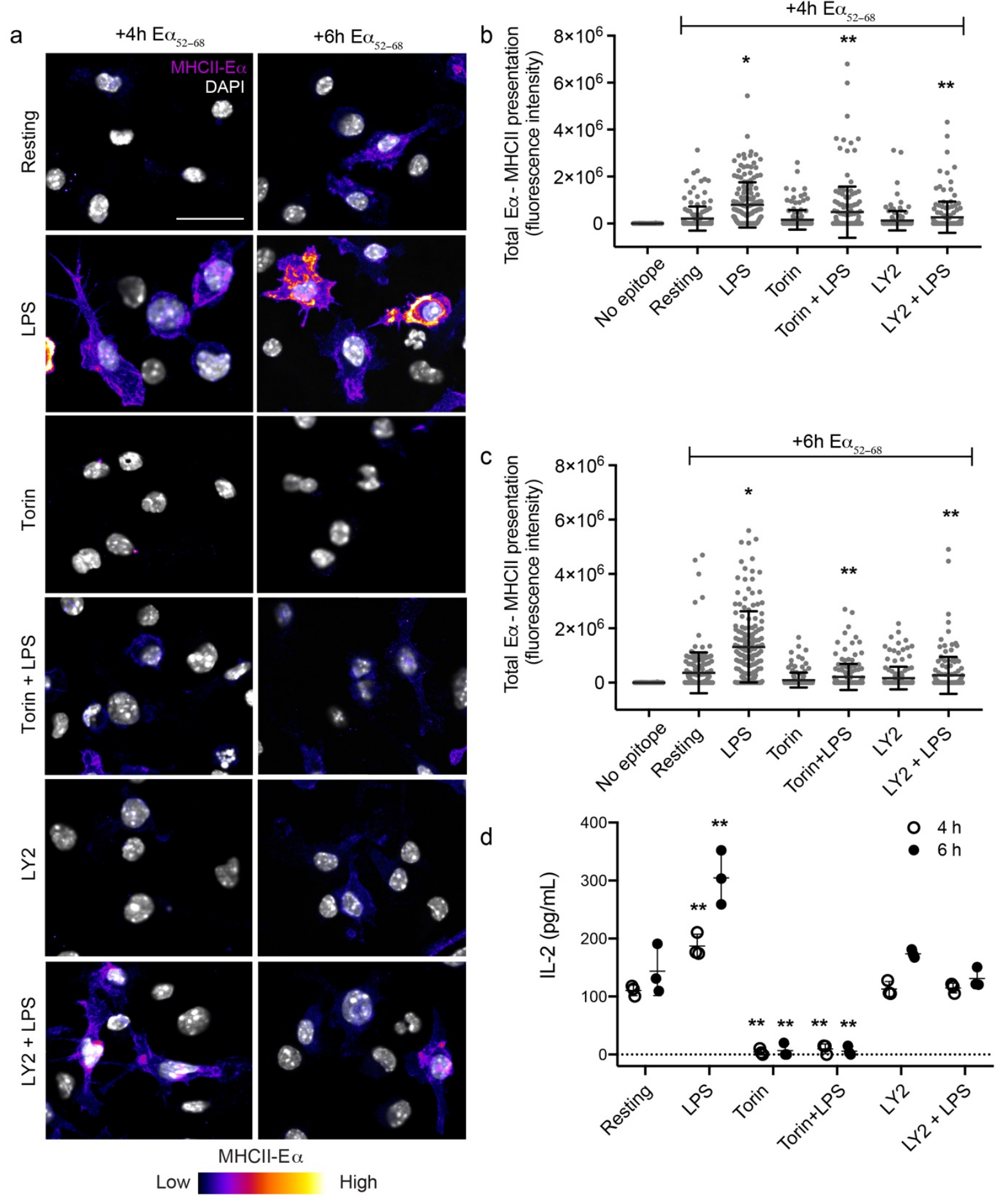


Figure 4.12: mTOR and S6 kinase control Ea $\alpha_{52-68}$ peptide presentation in activated BMDCs.

BMDCs were incubated with $\mathrm{E} \alpha^{52-68}$ peptide for 4 or 6 hours in the presence or absence LPS with or without torin 1 and LY258470. Cells were then fixed and stained with Y-Ae antibodies to detect

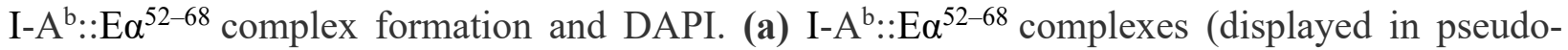
colour) and DAPI (grayscale) are shown for BMDCs treated as indicated. (b, c) Anti-I-A ${ }^{\mathrm{b}}:: \boldsymbol{E}^{52-}$ ${ }^{68}$ antibody signal was quantified by fluorescence intensity associated with each cell. Shown is the mean of the total fluorescence intensity of $\mathrm{I}-\mathrm{A}^{\mathrm{b}}:: \mathrm{E} \alpha^{52-68}$ complexes \pm SD from three experiments, where 50-100 cells were quantified for each. (d) BMDCs were fed $\mathrm{E} \alpha^{52-68}$ peptide for 4 or 6 hours in the presence or absence LPS with or without torin1 and LY258470. Following mild fixation, APCs were co-incubated with T-cells to measure I- $\mathrm{A}^{\mathrm{b}}:: \mathrm{E}^{52-68}$ complex induced T-cell activation. T-cell secreted Il-2 was measured using an ELISA system. All data were analysed using ANOVA, whereby * indicates a difference compared to unstimulated BMDCs exposed to $\mathrm{E} \alpha^{52-68}$ and ** indicates a difference compared to LPS-stimulated BMDCs fed $\mathrm{E}^{52-68}(\mathrm{p}<0.05)$. Scale bar $=30$ $\mu \mathrm{m}$. Colour scale: $0-2500$ (low-high). Figure A9 shows similar data for HEL presentation. 
Chapter 5: Lysosome adaptation in adjuvant-activated antigen presenting cells 


\subsection{Chapter rationale and overview}

Antigen presenting cells (APCs) like macrophages and dendritic cells reserve a specialized function to internalize and digest pathogens to generate antigens. Antigen processing occurs in the lysosome-related organelle referred to as the MHC-II compartment (ten Broeke, et al., 2013). Together with the work I presented in Chapter 4 and research from our lab and others, it is well supported that the endo-lysosomal system in APCs undergo dramatic reorganization upon activation with bacterial products, interferons or phorbol esters. Research shows that tubular lysosomes deliver MHC-II-antigen complexes to the plasma membrane for presentation to $\mathrm{T}$ cells (Boes et al., 2003; Chow et al., 2002; Mrakovic et al., 2012; Saric et al., 2016; Swanson et al., 1985; Vyas et al., 2007). Inhibition of tubulation blunts MHC-II on the surface of APCs (Saric et al., 2016). The work I present in Chapter 4 illustrates that activation of BMDCs expands the lysosome holding capacity, volume and increases antigen presentation and T-cell priming. Together with the data presented in Chapter 4, we speculate that lysosome tubulation and expansion in APCs enhances antigen uptake and retention, processing and presentation, and boosts $\mathrm{T}$ cell priming.

We speculate that this remodelling process in APCs may play an important role for the immunostimulatory effects of adjuvant molecules used in vaccines today. Adjuvant formulations can be broadly categorized into carrier or immunomodulatory, the former carrying or creating a depot of antigens and the latter directly engaging immune receptors to recruit and stimulate immune cells (Gutjahr et al., 2016). Chemically, adjuvants can be aluminum salts, emulsion-based, lipid-based, or agonists of pattern-recognition receptors such as toll-like receptors (Fox, et al., 2014; Gutjahr et al., 2016; Reed, et al., 2016). Within the vaccine discovery sector, there is great interest in natural and synthetic toll-like receptor agonists as adjuvants since they strongly induce 
antibody titer and quality, immune memory, type of immunity, and can reduce the antigen dose within a vaccine needed to develop immunity (Gutjahr et al., 2016). Adjuvant research is limited, there is a poor mechanistic understanding of how adjuvants actually trigger immunogenicity, how to classify adjuvants based on their signalling properties, and how to best couple specific antigens to specific adjuvants. However, there is growing interest in adjuvants that engage patternrecognition receptors like toll-like receptors, since they mimic natural MAMPs that the innate immune system recognizes as foreign (Fox et al., 2014; Gutjahr et al., 2016). We hypothesize that adjuvant molecules that are analogous to natural antigens may induce lysosome remodelling in APCs to boost the immunogenicity of vaccines and may serve as better adjuvant targets for vaccine design.

In this chapter, we present data that was completed in fulfillment of an OCE grant with an industry partner. All the data presented in this chapter was designed, completed and analyzed by me, unless otherwise stated. Due to possible non-disclosure agreements and patents, I am unable to disclose the names of the adjuvant molecules we tested. As a result, I will not be able to have in-depth discussions of the possible subcellular machinery and pathways that these molecules might trigger, other than what has already been introduced in Chapter 4 . The molecules we tested are currently found in vaccines used today, thus are of interest for our investigation to understanding the role of lysosome remodelling in phagocytes, and in identifying other agonists of lysosome remodelling. 


\section{$5.2 \quad$ Results}

\subsubsection{Select adjuvants can remodel the lysosome population}

We demonstrated that lysosome remodelling in phagocytes stimulated with LPS enhanced fluid phase uptake and accumulation, and bolstered antigen processing and presentation. To this end, we were interested in investigating whether lysosomal remodelling occurs in phagocytes stimulated with common adjuvants found in vaccines. We first tested whether select adjuvant compounds available to us were able to induce lysosome tubulation and remodelling. We obtained four compounds denoted as $\mathrm{A}, \mathrm{B}$ and $\mathrm{C}$, which are adjuvant molecules that mimic naturally occurring antigens, and compound $\mathrm{D}$, which is commonly used as an additive to complex with other adjuvant molecules.

We found that compounds $\mathrm{A}$ and $\mathrm{B}$ were able to induce lysosome tubulation in a dose dependent manner (Figure A10 a-d; Figure 5.1a). Whereas, compounds C and D did not induce lysosome tubulation (Figure A10 e; Figure 5.1a). Additionally, we tested whether these compounds signaled through mTOR near the optimal dose for lysosomal tubulation, and noticed a dose-dependent increase in mTOR activity through phosphorylation of its downstream target p70 S6K (Figure A10 e, f). Next, we were interested in whether the resulting increase in lysosomal tubulation corresponded with an enhancement in lysosomal volume. We found that both compounds A and B increased lysosomal volume in an mTOR-dependent manner, whereas compound C did not (Figure 5.1b). Unfortunately, we could not measure lysosome volume in cells treated with compound $\mathrm{D}$, as it was auto-fluorescent in all channels, which would confound the volumetric analysis we employ (Figure A10g).

Since both compounds A and B increased lysosomal tubulation through mTOR, we next measured whether these cells were able to accumulate more fluid phase material as we saw in LPS stimulated cells (Figure 4.3). We found that cells stimulated with compound A internalized more 
fluid phase material compared to resting cells consistent with LPS stimulated cells (Figure A10h). Interestingly, we did not see the same increase in fluid phase accumulation when using compound B (Figure A10i). This could be due to the differential functions of remodelling between the two compounds, temporal differences in activation and/or the assay we used for this experiment. Nonetheless, our data thus far suggest that both compounds A and B were able to induce lysosomal remodelling through mTOR, which provides insight into the possible cellular mechanism by which these adjuvants bolster immunity upon vaccination. 


\subsubsection{Adjuvants bolster antigen presentation through mTOR and S6K}

Thus far, we have shown that compounds $\mathrm{A}$ and $\mathrm{B}$ can induce lysosome remodelling by inducing lysosome tubulation and increasing lysosomal volume through mTOR activity. We were next interested in whether these compounds boost antigen presentation through mTOR activity. It is has already been extensively reported that many adjuvants can boost antigen presentation, albeit most literature investigates this response following long-term activation. To this end, we fed BMDCs with Hen-egg lysozyme (HEL) for 6 hours in the presence and absence of each adjuvant. For

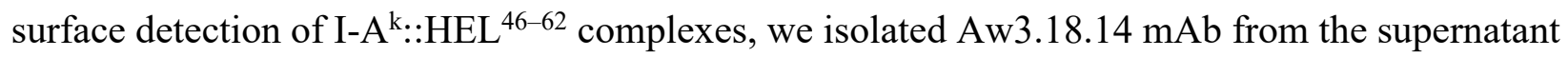
of hybridoma B-lymphocytes. Briefly, we incubated treated cells with Aw3.18.14 mAb on ice, to only detect surface levels of the antigen-MHCII complex, then fixed cells and continued with standard immunofluorescence protocols. We found that both compounds A and B significantly increased MHCII I-A ${ }^{\mathrm{k}:: H E L}{ }^{46-62}$ complexes on the plasma membrane compared to resting cells (Figure 5.2a, b). Moreover, this process was mTOR-dependent, as treatment with Torin1 blocked HEL-MHCII complex accumulation at the plasma membrane (Figure 5.2a, b).

In addition to using HEL as model antigen, we also used purified Ea52-68 peptide and the corresponding YAe antibody, which specifically recognizes MHCII- I-A $A^{\mathrm{b}}:: \mathrm{E} \alpha_{52-68}$ complexes. As we found with HEL presentation, compounds A and B significantly bolstered MHCII- I-A $A^{\mathrm{b}:: E \alpha_{52}-}$ 68 complex formation in cells (Figure 5.3a, b). When we blocked mTOR or S6K activity using Torin1 and LY258470, we found that antigen complex formation in cells was blunted, further supporting a role for mTOR and lysosomal remodelling in antigen presentation following adjuvant stimulation.

Compound $\mathrm{D}$ is commonly used in vaccines in combination with other adjuvants to further enhance the immunostimulatory effect of adjuvants. To this end, we tested whether compound D 
alone enhanced antigen presentation in BMDCs. We found that there was no change in MHCII- I-

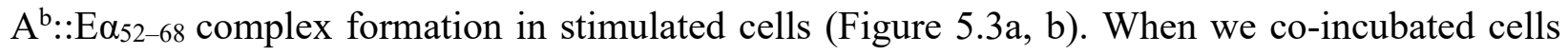
with compounds $\mathrm{A}$ and $\mathrm{D}$ or compounds $\mathrm{B}$ and $\mathrm{D}$, we saw an interesting effect on antigen presentation. Specifically, combining compounds A and D did not significantly enhance antigen presentation compared to compound A alone (Figure 5.4a-c). However, when combining compounds B and D, we saw a significant increase in total MHCII- I-A $\mathrm{A}^{\mathrm{b}}:$ E $\alpha_{52-68}$ complex formation compared to compound B alone (Figure 5.4a, b, d). To investigate the possible effect that combining adjuvants may have on the distribution of cells in the population undergoing MHCII- I-A ${ }^{\mathrm{b}}:: \mathrm{E}_{52-68}$ complex formation, we binned the fluorescent measurement data in (Figure 5.4b). As demonstrated with compound A and D combinations, there was no observable shift in the bin groups between the combined group and compound A alone (Figure 5.4c). However, when compounds B and D were complexed, the lower bin distribution decreased in comparison to compound B alone and the distribution of higher bins was slightly shifted towards the left (Figure 5.4d). Together, this distribution suggests that combining the adjuvants may shift the overall population towards more cells undergoing antigen presentation and further enhance those that are, in comparison to resting and those cells treated with B alone (Figure 5.4d). These data are interesting, as they suggest that when we combine adjuvants, we may have the ability to further enhances the effect of a single adjuvant. Though more insight into the mechanisms underlying adjuvant combinations is needed. 


\subsubsection{Adjuvants bolster T-cell activation through mTOR and S6K}

Thus far, we have shown that compounds A and B are able to increase antigen presentation and lysosomal remodelling and that adjuvant combination may further enhance the immunostimulatory effect of some adjuvants. Next, we were interested in investigating whether adjuvant-induced lysosome remodelling in BMDCs increased T-cell activation. As previously described, we took pre-activated BMDCs and co-incubated them with T-cells for 40 hours. We measured the amount of secreted IL-2 as a measure of T-cell activation. When BMDCs were pre-treated with compound A we saw a significant increase in the amount of secreted IL-2 compared to resting cells (Figure 5.5a). When we blocked lysosome remodelling in BMDCs during phagocyte activation using Torin1 and LY258470, we blunted the effects of compound A remodelling of BMDCs on T-cell activation (Figure 5.5a). When we co-incubated compound A and D, there was no significant increase in T-cell activation, suggesting the combination did not significantly impact the immunostimulatory effect of compound A (Figure 5.5a).

Similarly, we looked at the impact of T-cell activation in BMDCs pre-activated with compound B. Interestingly, when we fed BMDCs Eas2-68 peptide and activated them using compound B, T-cell secretion of IL-2 did not significantly increase (Figure 5.5b). However, when we co-incubated BMDCs with compounds B and D, there was a significant enhancement in secreted IL-2 compared to resting and compound B alone treatment (Figure 5.5b). These data suggest that pre-activation of BMDCs with compound B alone was not sufficient to prime and activate T-cells, rather co-stimulation with compound D bolsters the effect of compound B on T-

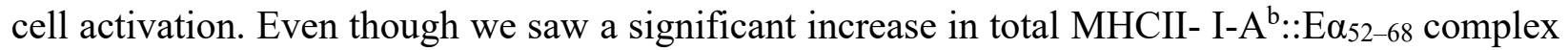
formation following compound B treatment and no effect on T-cell activation, it may be that compound B alone is increasing antigen-MHCII complex formation but it is not sufficient for 
efficient presentation at the plasma membrane, and instead compound D combination may catalyze effective antigen presentation. Altogether, our data suggests that some adjuvants induce lysosomal remodelling through mTOR and S6K activity, which is essential for optimal antigen presentation and T-cell activation. 


\section{$5.3 \quad$ Figures}


a
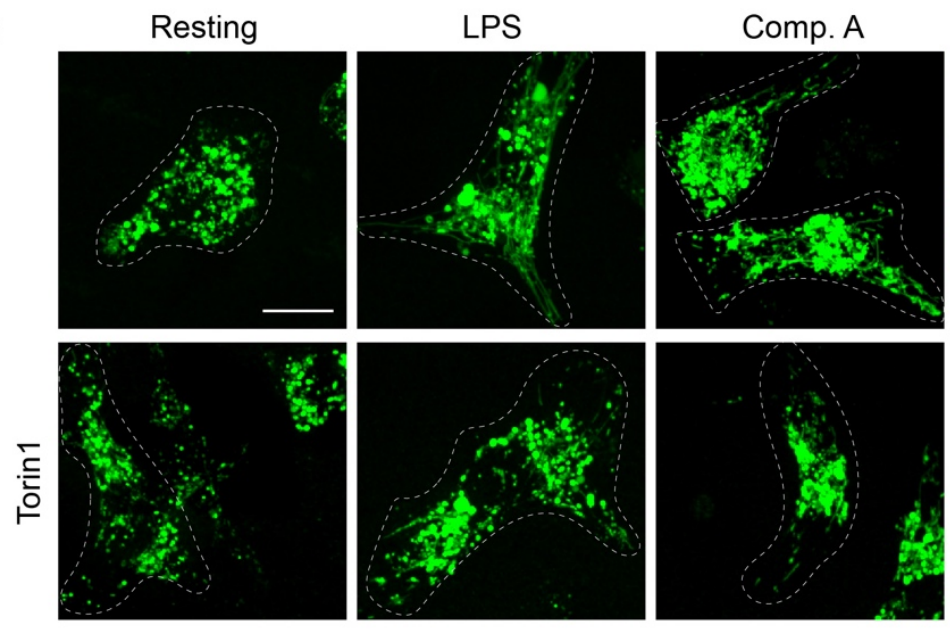

Comp. B
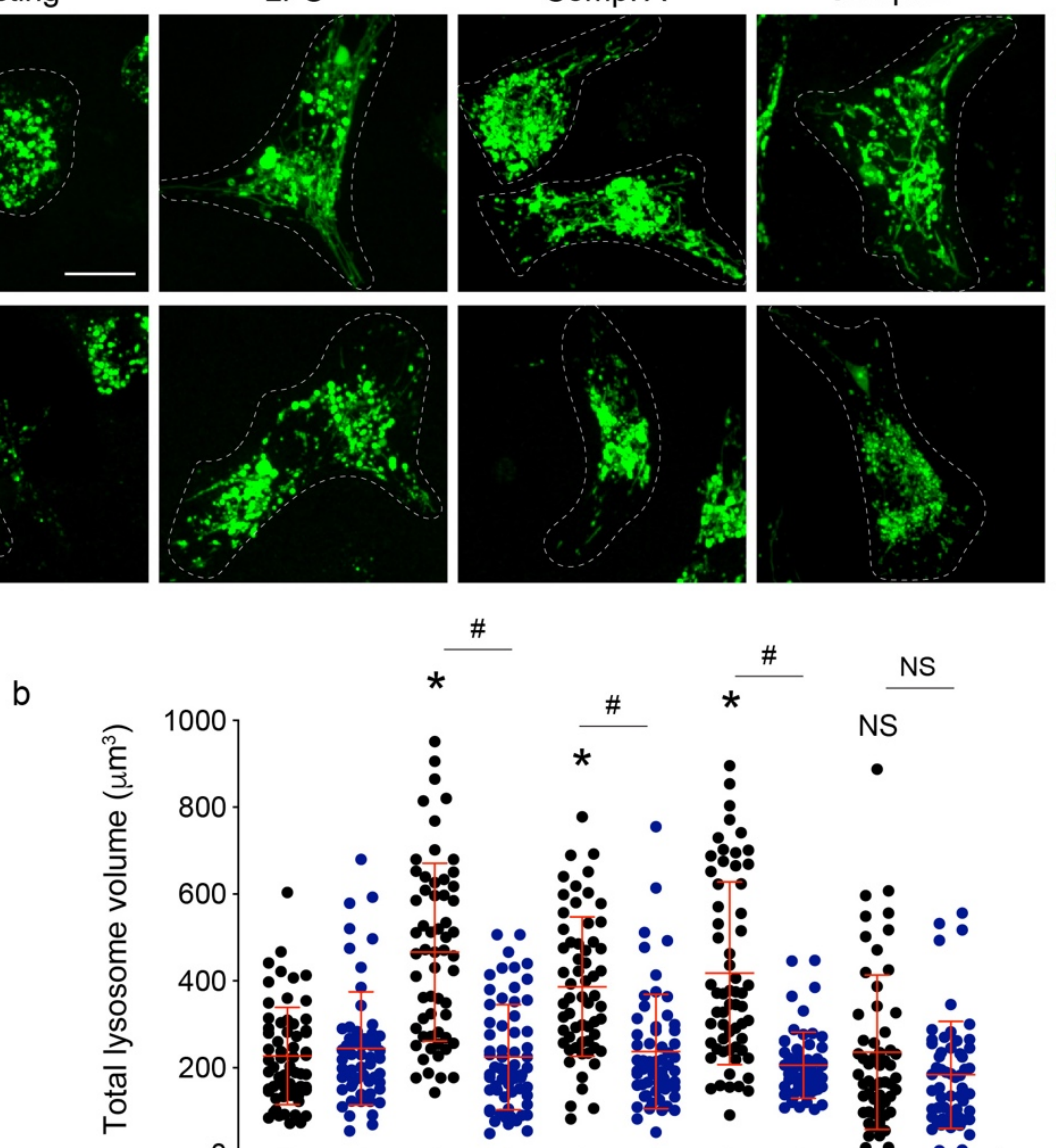
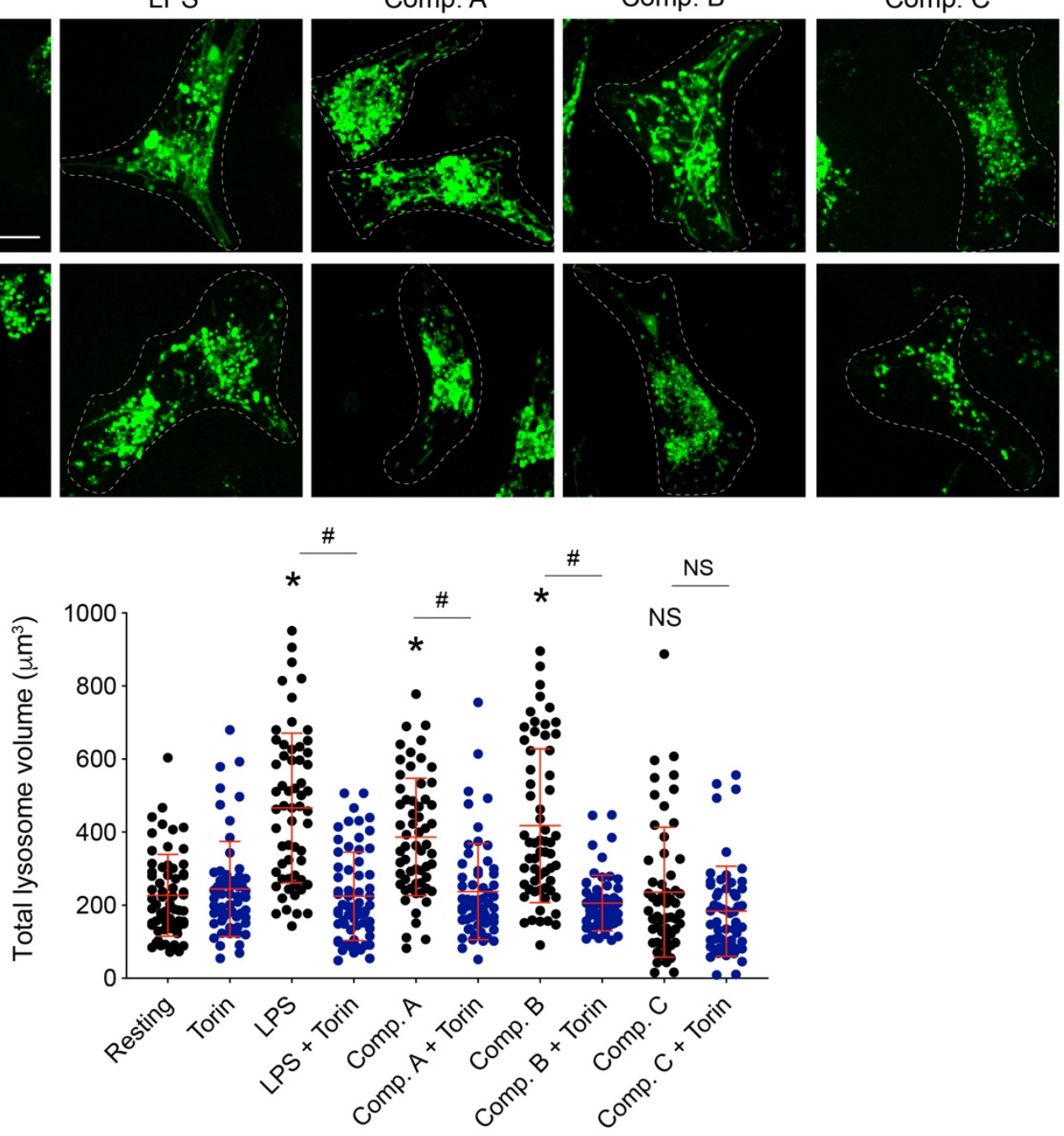

Figure 5.1: Select adjuvants induce lysosome remodelling through mTOR.

(a) Lysosomes in resting, LPS and adjuvant-stimulated BMDCs after $2 \mathrm{~h}$ stimulation and/or upon mTOR inhibition with Torin1. Compounds A and B induce lysosomal tubulation in an mTORdependent manner. (b) Total lysosomal volume in BMDCs treated in a. Adjuvants remodel the lysosome population by increasing lysosomal volume through mTOR activity. All experiments were repeated at least three independent times. Data are based on 30-40 cells per condition per experiment and are shown as the mean $\pm \mathrm{SD}$. Statistical analysis was completed using one-way ANOVA and unpaired Student's t-test, where the asterisk * indicates a significant increase compared to resting phagocytes, and \# compared to the test compound $(\mathrm{p}<0.05)$. Scale $=10 \mu \mathrm{m}$. 
Figure 5.2

a
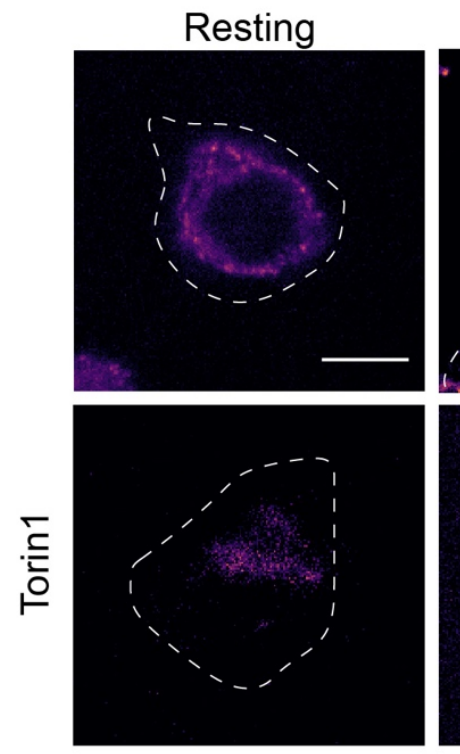
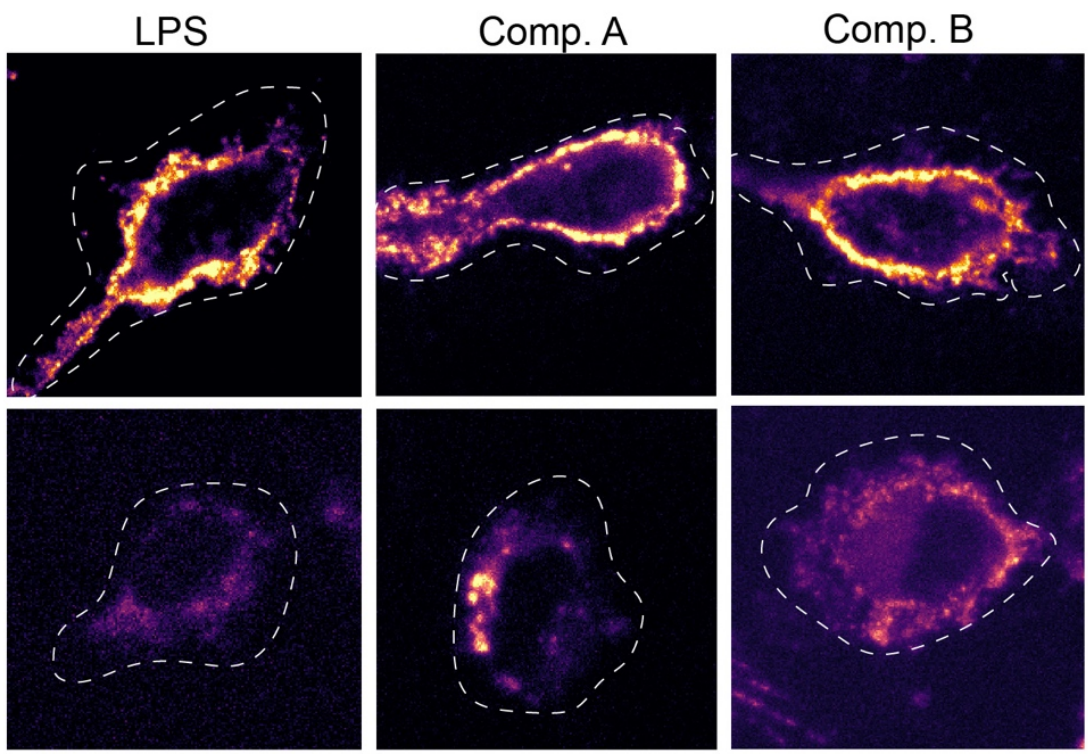

MHCII-HEL

Low

High

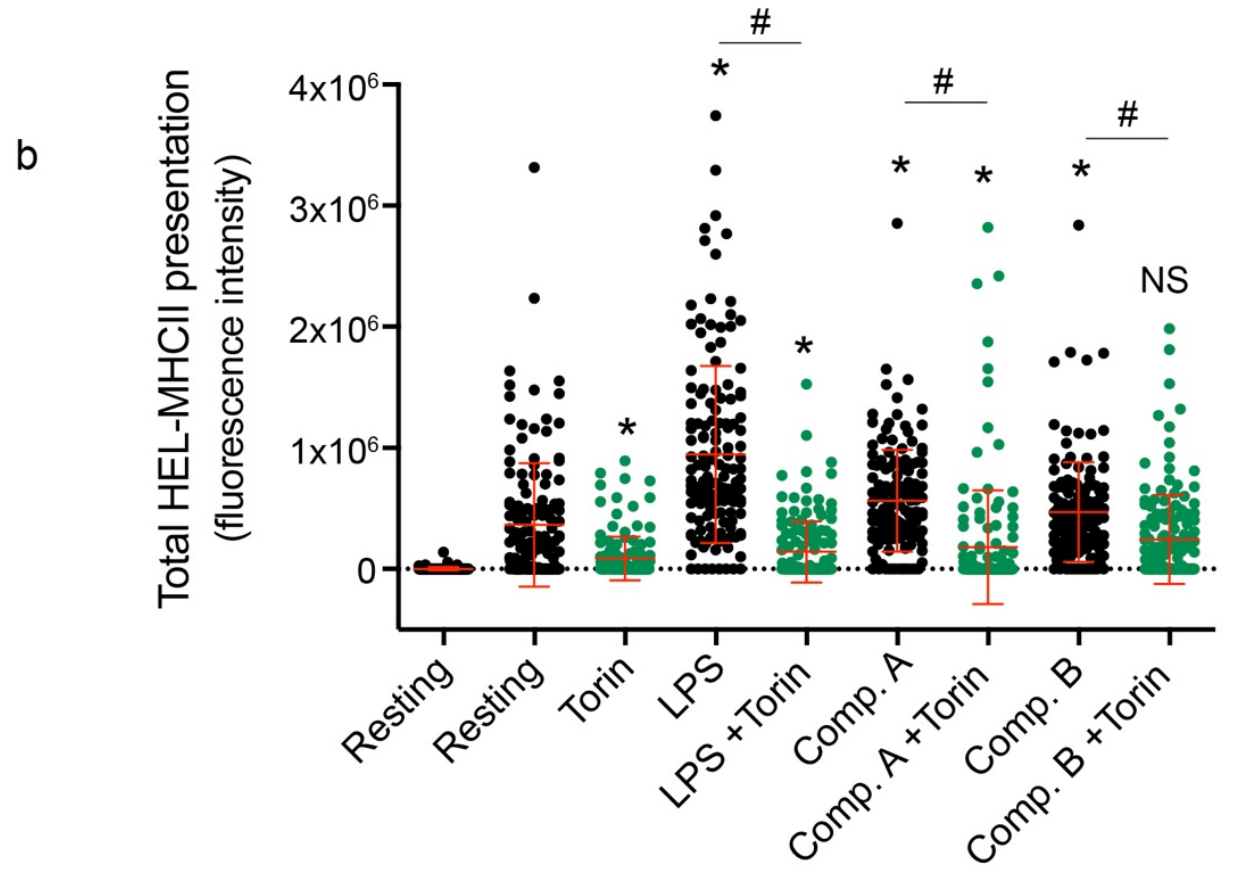


Figure 5.2: Compound A and B enhance HEL-MHCII presentation through mTOR.

(a) I-A $\mathrm{A}^{\mathrm{k}:: H E L^{46-61}}$ presentation in BMDCs after incubation with HEL for 6 hours in the presence and/or absence of LPS, adjuvant compounds and Torin1. I-A $::$ HEL $^{46-61}$ cell surface levels were detected by staining unpermeabilized cells with the monoclonal antibody Aw3.18.14 (b)

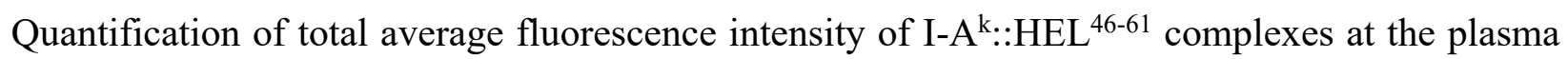
membrane. Shown is the mean \pm SD from three experiments, where 50-100 cells were quantified for each. Data was analyzed using ANOVA, whereby * indicates a difference compared to the Resting + HEL condition and \# indicates a difference compared to the corresponding test compound $(\mathrm{p}<0.05)$. Scale bar $=15 \mu \mathrm{m}$. Colour scale: $0-12000$ (low-high). 
Figure 5.3
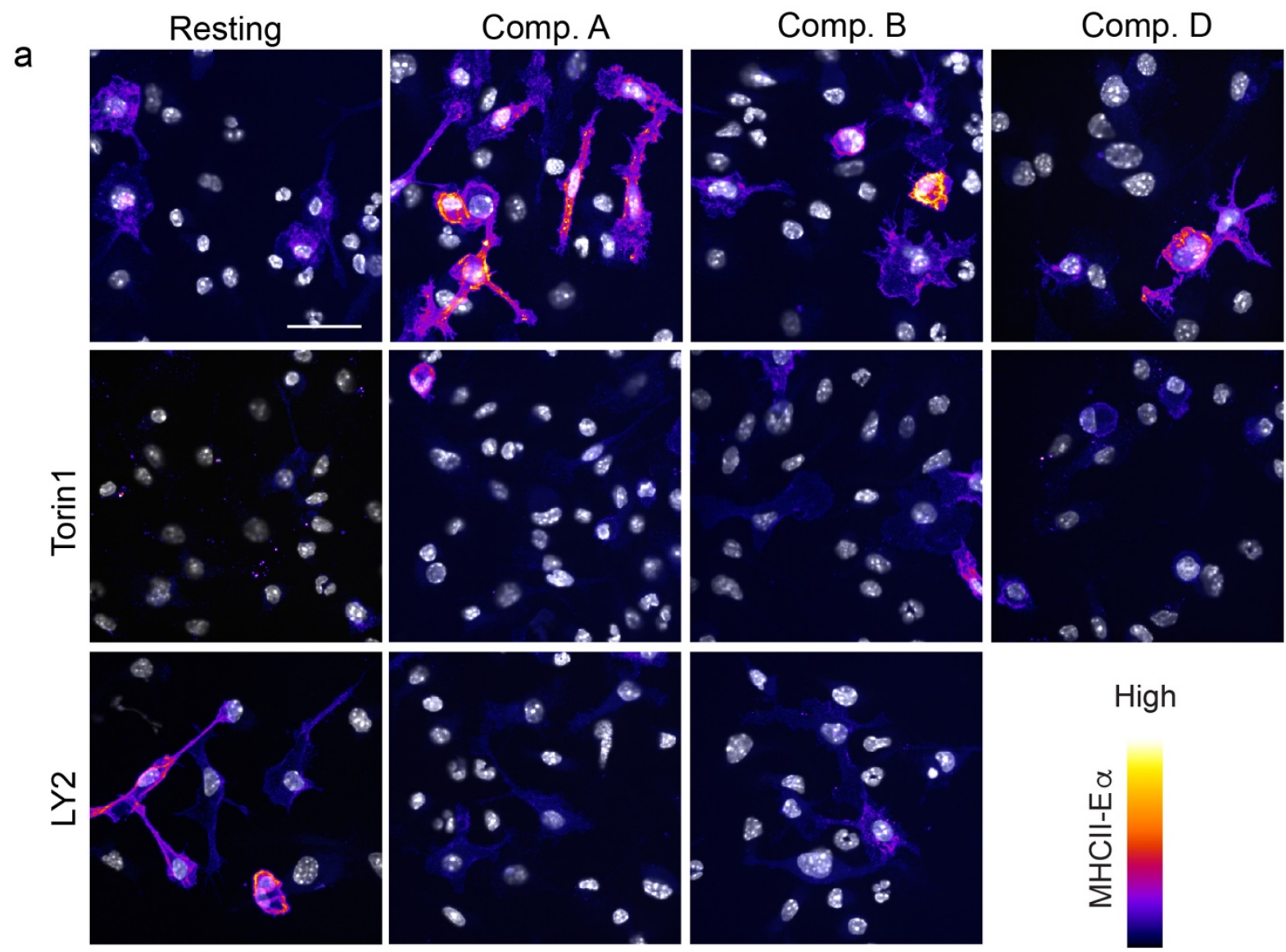

High

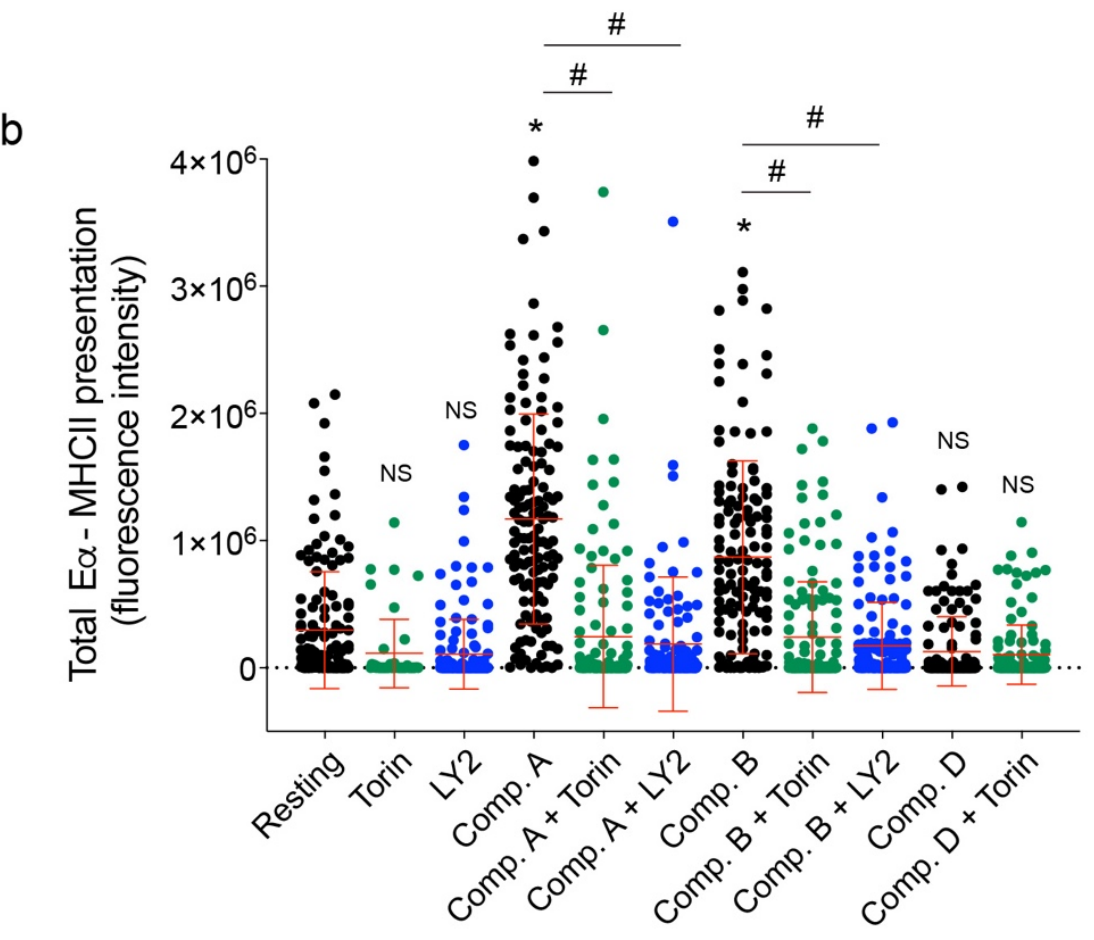




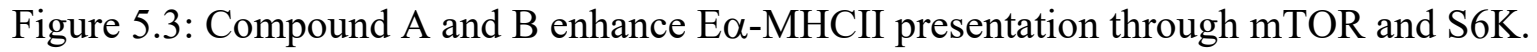

(a) BMDCs were incubated with $\mathrm{E} \alpha^{52-68}$ peptide for 6 hours in the presence or absence of adjuvant compounds, with or without torin 1 and LY258470. Cells were then fixed and stained with Y-Ae

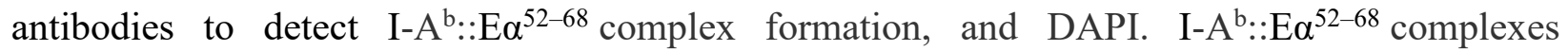
(displayed in pseudo-colour) and DAPI (grayscale) are shown for BMDCs treated as indicated. (b) Anti-I-A ${ }^{\mathrm{b}}:: \mathrm{E}^{52-68}$ antibody signal was quantified by fluorescence intensity associated with each cell. Shown is the mean of the total fluorescence intensity of $\mathrm{I}-\mathrm{A}^{\mathrm{b}}:: \mathrm{E} \alpha^{52-68}$ complexes $\pm \mathrm{SD}$ from three experiments, where 50-100 cells were quantified for each. Data was analysed using ANOVA, whereby * indicates a difference compared to stimulated BMDCs exposed to E $\alpha^{52-68}$ and ** indicates a difference between test compound treatment $(\mathrm{p}<0.05)$. Scale bar $=30 \mu \mathrm{m}$. Colour scale: $0-2500$ (low-high). 
Figure 5.4
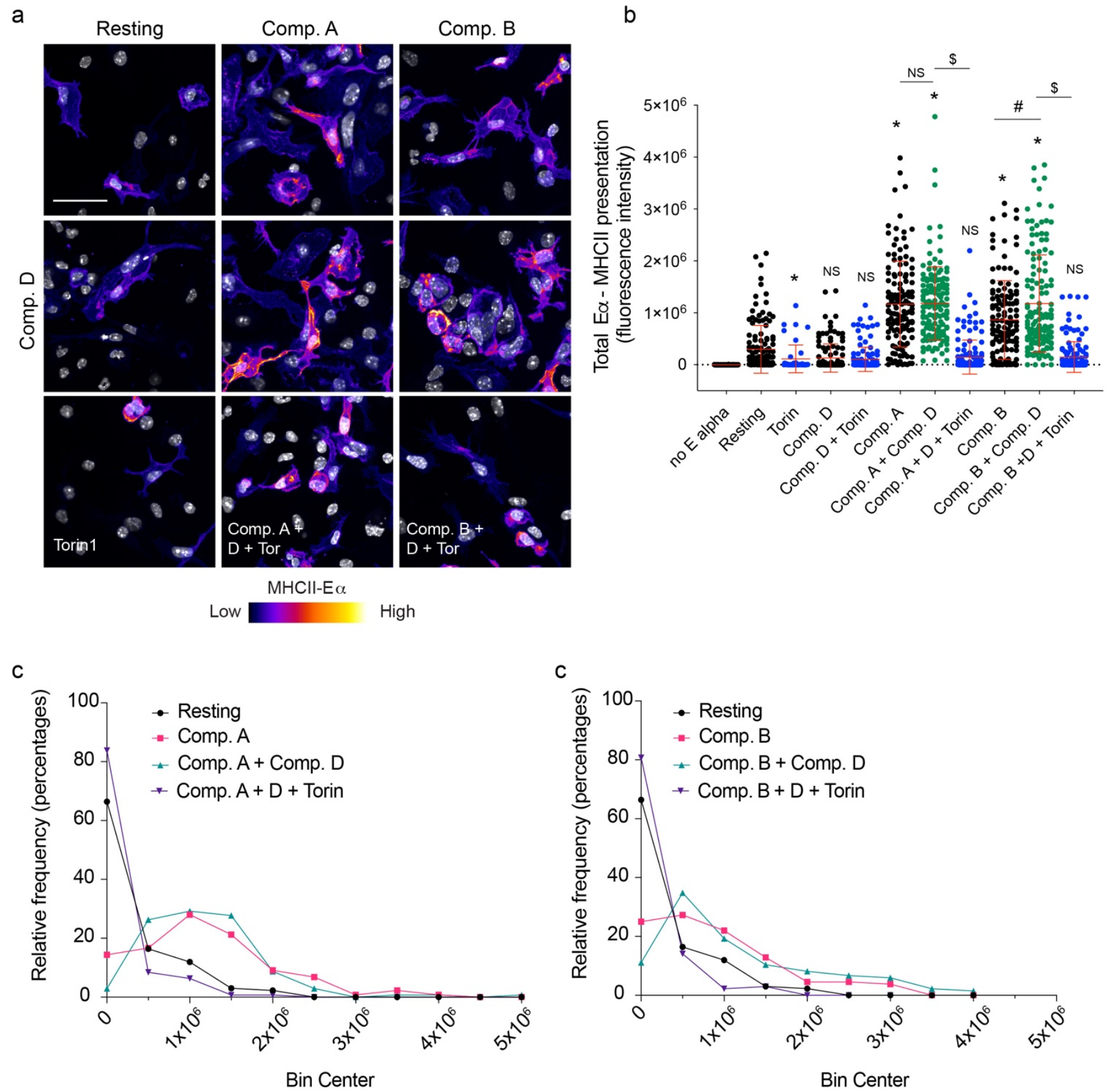
Figure 5.4: Adjuvant complexing can enhance Ag-presentation efficacy.

(a) BMDCs were incubated with $\mathrm{E} \alpha^{52-68}$ peptide for 6 hours in the presence or absence adjuvant compounds alone or complexed, with or without torin1. Cells were then fixed and stained with YAe antibodies to detect I-A $A^{\mathrm{b}}:: E \alpha^{52-68}$ complex formation and DAPI. I-A ${ }^{\mathrm{b}:: E \alpha^{52-68}}$ complexes (displayed in pseudo-colour) and DAPI (grayscale) are shown for BMDCs treated as indicated. (b)

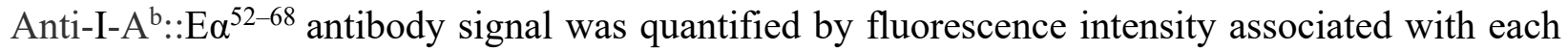
cell. Shown is the mean of the total fluorescence intensity of $\mathrm{I}-\mathrm{A}^{\mathrm{b}}:: \mathrm{E}^{52-68}$ complexes $\pm \mathrm{SD}$ from three experiments, where 50-100 cells were quantified for each. Data was analysed using ANOVA, whereby * indicates a difference between resting and compound-stimulated BMDCs exposed to $\mathrm{E} \alpha^{52-68}$, \# indicates a difference between compound treatment and complexed adjuvant, and $\$$ indicates a difference between complexed adjuvant and Torin1 treatment $(\mathrm{p}<0.05)$. Scale bar $=30$ $\mu \mathrm{m}$. Colour scale: $0-2500$ (low-high). (c) Quantified data from b illustrated with a binned distribution plot to demonstrate population shifts between adjuvant and combined adjuvant groups. Compound A data (left) and compound b data (right). Bins were automatically generated using Prism graphing software. 
a

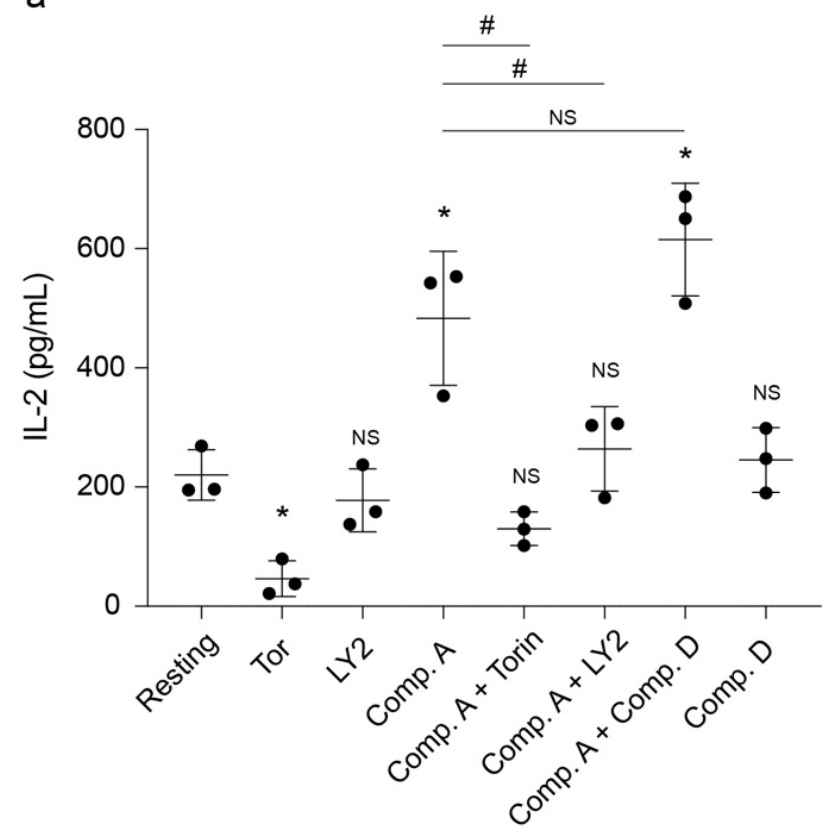

b
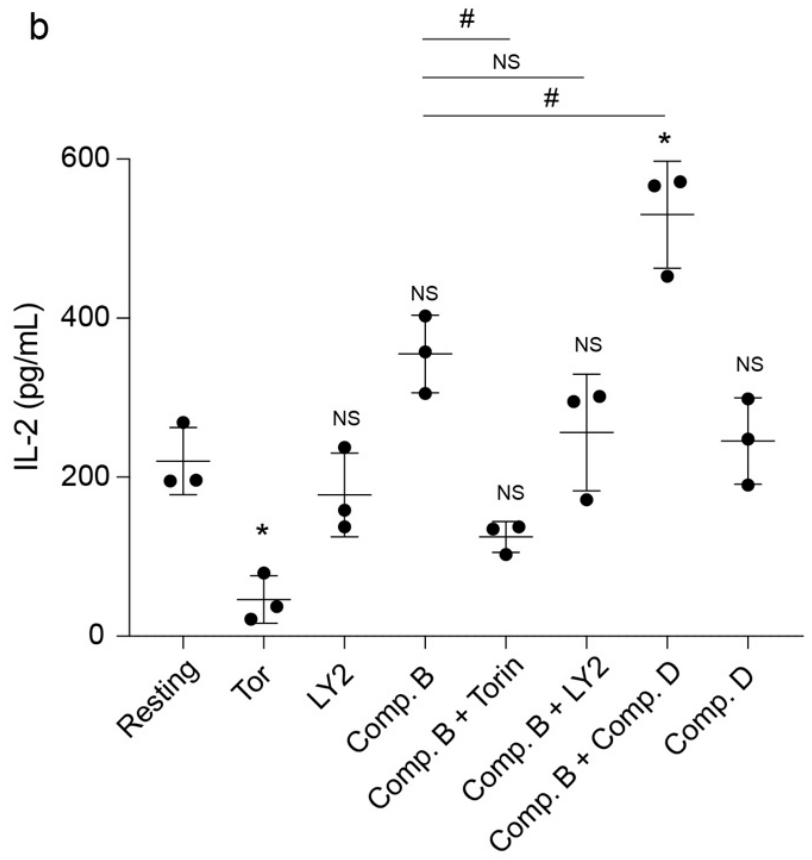

Figure 5.5: Lysosome remodelling in adjuvant-activated DCs enhances T-cell activation.

(a,b) BMDCs were fed $\mathrm{E}^{52-68}$ peptide for 6 hours in the presence or absence compound A (a) or compound B (b) with or without Torin1 and complexed with compound D. Following mild fixation, APCs were co-incubated with T-cells to measure I-A $\mathrm{A}^{\mathrm{b}}: \mathrm{E} \alpha^{52-68}$ complex induced T-cell activation. T-cell secreted Il-2 was measured using an ELISA system. All data was analysed using ANOVA, whereby * indicates a difference between resting and stimulated BMDCs exposed to $\mathrm{E} \alpha^{52-68}$ and \# indicates a difference between the corresponding compound treatment $(\mathrm{p}<0.05)$. 
Chapter 6: Discussion 


\subsection{The tubular phagocytic cup as a model to study phagosome maturation}

The stages of phagosome maturation are well-studied in cellular biology. A significant amount of research utilizes spheroidal targets to better understand the molecular machinery that drives each stage. As described in the introduction, in nature immune cells are challenged with targets of disparate shapes. Using fixed-filamentous bacterium to form a long tPC, in Chapter 3 I presented three main aspects in $\mathrm{TPC}$ biogenesis that contravene the canonical maturation pathway of phagosomes. Briefly, we identified that endosomal and lysosomal markers are recruited to the phagocytic cup prior to its scission from the plasma membrane, which is uncoupled from the acidification of the compartment. Second, whereas the early phagosomal proteins Rab5 and EEA1 arrive timely to growing tPCs, and disappear as the phagocytic cups elongate, PtdIns(3)P persists on tPCs. Third, PtdIns(3)P overlaps with endolysosomal markers as long as the $\mathrm{pH}$ of the tPCs

remains near neutral. Herein, I discuss the three main aspects of tPC maturation and their implications in better understanding the stages of phagosome maturation. Additionally, I propose future work to study the stages of maturation using the tubular phagosome as a model which offers both temporal and spatial resolution of phagocytosis.

\subsubsection{PtdIns(3)P remains on tPCs regardless of other early and late endosomal markers}

Canonical phagocytosis of spherical targets indicates that early endosomal markers Rab5, EEA1 and PtdIns(3)P are acquired following the scission and formation of a nascent phagosome (Flannagan, Jaumouillé, \& Grinstein, 2012). Additionally, it is well understood that the late endosomal markers Rab7 and LAMP1 are recruited upon the loss of the earlier markers (Botelho \& Grinstein, 2011; Vieira et al., 2002). When we investigated the fate of early and late endosomal markers on the growing tubular phagocytic cup, both early (Rab5, EEA1 and PtdIns(3)P) and late 
endosomal markers (LAMP1 and Rab7) were localized to the same growing tubular phagocytic cup prior to its closure and scission from the plasma membrane (Figure 3.1). When we followed the life-time of these markers over 30 minutes of uptake, the entire tPC lacked Rab5 and EEA1, but contained the late markers LAMP1 and Rab7, akin to the canonical model, in which early markers are replaced by late markers. Additionally, tPC length also dictated the localization of endosomal markers; on the same tPC, areas most proximal to the plasma membrane were positive for early endosomal markers and the most distal portions were enriched with late endosomal markers.

PtdIns(3)P is a critical regulator of endosome and phagosome maturation, orchestrating many signalling events. Localized synthesis of PtdIns(3)P allows for the recruitment of a number of adaptor proteins like EEA1 and Hrs (hepatocyte growth factor-regulated tyrosine kinase substrate) onto endosomes and phagosomes to regulate membrane fusion and recycling, as well as regulating protein sorting to the Golgi and multivesicular bodies (Levin, Grinstein, \& Schlam, 2015; Schink, Raiborg, \& Stenmark, 2013). While PtdIns(3)P synthesis is necessary for endosome and phagosome maturation, PtdIns(3)P turnover is also considered a hallmark in the maturation process. Interestingly, when we tracked PtdIns(3)P using fluorescently-tagged 2FYVE and p40PX, both markers persisted on tPCs despite a divestment of other early endosomal markers and the acquisition of endo-lysosomal proteins (Figure 3.1), suggesting that signals that normally couple PtdIns(3)P removal with maturation fail on tPCs.

As established with canonical phagosomes, the recruitment and retention of PtdIns(3)P at tPCs may result either from (i) excessive synthesis mediated by phosphoinositide-3 kinases (PI3Ks) or (ii) inhibition of its conversion/degradation via PIKfyve and/or myotubularins (Cao, Backer, Laporte, Bedrick, \& Wandinger-Ness, 2008; Hazeki et al., 2012; Ho et al., 2012; Jin, Lang, 
\& Weisman, 2016; Kim et al., 2014; McCartney et al., 2014). We investigated the former and determined that Class I PI3Ks were not involved in the synthesis on tPCs. Rather, PtdIns(3)P retention at tPCs was a result of synthesis by the Class III PI3K Vps34 (Vieira et al., 2001) (Figure 3.2). Indeed, inhibition of Vps34 depleted PtdIns(3)P on tPCs to an undetectable level. To examine PtdIns(3)P turnover, we investigated the role of PIKfyve, which phosphorylates PtdIns(3)P to produce PtdIns(3,5) $\mathrm{P}_{2}$ (Ho, Alghamdi, \& Botelho, 2012; McCartney, Zhang, \& Weisman, 2014). We found that inhibition of PIKfyve using apilimod strongly increased PtdIns(3)P accumulation onto tPCs. Together, these data suggest that PtdIns(3)P maintenance on tPCs is due to synthesis by Vps34, rather than a defect in its turnover. Interestingly, PtdIns(3)P was eventually eliminated by PIKfyve upon complete enclosure of the filamentous target or from the tPC regions that extended beyond a length of $20 \mu \mathrm{m}$, indicating that tPCs were competent for canonical turnover under length-dependent conditions (Figure 3.3).

\subsubsection{Phagosome acidification controls Vps34 complex II association and PtdIns(3)P levels}

Next, we investigated the interplay between PtdIns(3)P loss and acidification in tPCs, canonical phagosomes, as well as endosomes. Acidification of these compartments is a hallmark of endosome and phagosome maturation. Briefly, using live-cell microscopy and biochemical techniques, we resolved a pH-dependent mechanism that controls Vps34 complex II association with phagosomal membranes and PtdIns(3)P levels. Using pHRodo-conjugated filamentous bacteria, spheroidal phagosomes and zymosan particles, we found that $\operatorname{PtdIns}(3) \mathrm{P}$ markers dissociated from maturating phagosomes only upon luminal acidification (Figure 3.4). When we forced luminal acidification and neutralization of the phagosome PtdIns(3)P markers dissociated from and persisted on the phagosomal membranes, respectively (Figure 3.6 \& Figure 3.7). 
Following internal $\mathrm{pH}$ calibration experiments for several tPCs and phagosomes, we determined that a luminal $\mathrm{pH}$ between 6.1-6.3 triggers PtdIns(3)P loss from tPC and canonical phagosomal membranes (Figure 3.5 \& Figure 3.8). Our data provide a novel mechanism indicating that PtdIns(3)P levels are controlled through a $\mathrm{pH}$-dependent mechanism on maturing phagosomes. In addition, it goes against the canonical view of PtdIns(3)P turnover, in which it was suggested that phagosomal loss of early endosomal markers precedes luminal acidification, as it is accepted that the maturing phagosome undergoes acidification upon fusion with late endo-lysosomal compartments (Botelho \& Grinstein, 2011; Flannagan et al., 2012; Vieira et al., 2002). In contrast, our data suggest that the early phagosome may undergo mild acidification prior to fusion.

We were interested in investigating whether the Vps34 complex II machinery responded to phagosomal luminal $\mathrm{pH}$ to control PtdIns(3)P levels, and measured its association on isolated spheroidal phagosomes following forced acidification or alkalization. We found that subunits of the Vps34 complex (Vps15, UVRAG and Vps34) responded to luminal pH. Indeed, acidification or neutralization with ammonium chloride caused the subunits to disassociate or remain on the phagosomal membranes, respectively (Figure 3.9). Unfortunately, we were unable to optimize immunofluorescence staining of endogenous Vps34 complex II subunits with the antibodies currently available. In the future, it would be interesting to probe for their association with tPCs and to resolve whether their recruitment is associated with the $\mathrm{pH}$ gradient we observed in tPCs. If their recruitment was dependent on $\mathrm{pH}$, we would expect to see a similar gradient as observed with luminal $\mathrm{pH}$ for tPCs longer than $20 \mu \mathrm{m}$ in length. In addition, it would be interesting to obtain fluorescently-tagged constructs of Vps34, or CRISPR-edited cell-lines expressing tagged-subunits (Stjepanovic, Baskaran, Lin, \& Hurley, 2017; Su et al., 2017), and probe for the localization and activity of Vps34 in response to changes in pH. Furthermore, to better understand how Vps34 
and/or its subunits respond to or are modified in response to changes in luminal $\mathrm{pH}$, we could employ co-immunoprecipitation techniques using tandem affinity purification (TAP) followed by mass spectrometry (Dalvai et al., 2015; Rigaut et al., 1999). Using CRISPR editing technology, we could endogenously tag Vps34 and/or subunits to purify native protein complexes, then use mass spectrometry to analyze protein-protein interactions and post-translational modifications acquired in response to changes in $\mathrm{pH}$.

Altogether our data supports that acidification of the phagosomal lumen signals the cessation of PtdIns(3)P synthesis by triggering the dissociation of the Vps34 complex II subunits from membranes. Overall, we present evidence of a novel $\mathrm{pH}$-based mechanism that modulates the association of Vps34 with endo-membranes, controlling PtdIns(3)P synthesis (modeled in Figure 6.1).

\subsubsection{How can luminal pH control Vps34 association onto phagosomal membranes?}

In Chapter 3, I present data supporting a novel mechanism in which phagosome/endosome acidification during maturation, induces a $\mathrm{pH}$-gradient across the membrane that serves as a trigger to displace Vps34 complex II from membranes. One mechanism by which pH can control Vps34 association is through the action of a $\mathrm{pH}$-sensitive transmembrane protein, which may act as a sensor for changes in luminal $\mathrm{pH}$. This sensor may exhibit a $\mathrm{pH}$-dependent conformational change which is transmitted to onto the cytoplasmic face of the phagosome and signals to Vps34 and/or its subunits. Such $\mathrm{pH}-$ dependent conformational changes are seen in several proteins, such as in the viral envelope protein, hemagglutinin which undergoes a $\mathrm{pH}$-dependent conformational change to facilitate viral entry into the endosomal membrane and is essential for influenza infection (Kalani, Moradi, Moradi, \& Tajkhorshid, 2013). 
Additionally, our data showed that inhibition of the V-ATPase with concanamycin A, an inhibitor of proton translocation in subunit $\mathrm{C}$ of the $\mathrm{V}_{0}$ subunit hindering luminal acidification (Huss et al., 2002), altered PtdIns(3)P association onto tPC and canonical phagosome membranes. However, the V-ATPase itself may sense changes in $\mathrm{pH}$, as the a2-subunit of the endosomal VATPase isoform has been reported to function as a possible pH-sensor (Hurtado-Lorenzo et al., 2006; Marshansky, 2007; Smith et al., 2016). Additionally, the V-ATPase is well known to sense and transduce luminal concentrations of amino acids to regulate mTORC1 activity on lysosomal membranes during nutrient depleted conditions (Liron Bar-Peled \& Sabatini, 2014; Marshansky, Rubinstein, \& Grüber, 2014). Together these data suggest that the V-ATPase may serve as a pHsensor to control the association of Vps34 complex II onto phagosomal and endosomal membranes using an 'inside-out' mechanism, whereby it can transduce luminal signals into the cytosol and nearby proteins.

Another mechanism by which $\mathrm{pH}$ may regulate Vps34 complex II recruitment is through the diffusion of protons across endosomal and phagosomal membranes (Demaurex, 2002; Lukacs, Rotstein, \& Grinstein, 1990; Marshansky, 2007). Proton leakage driven by a variety of ion transporters may generate localized changes in the $\mathrm{pH}$ around endosomes and phagosomes that could be sensed by other $\mathrm{pH}$-sensitive signalling proteins in the cytoplasm (Casey, Grinstein, \& Orlowski, 2010; Johnson \& Casey, 2011; Koivusalo et al., 2010). For example, in order to maintain the electrochemical gradient across the endo-phagosomal membrane necessary to drive V-ATPase activity, select counterion channels may be active. A counterion channel candidate for endolysosomal organelles is the $\mathrm{ClC}-7 \mathrm{Cl}^{-} / \mathrm{H}^{+}$exchanger. This ion transporter moves two $\mathrm{Cl}^{-}$ions into the lumen and one $\mathrm{H}^{+}$proton into the cytosol to maintain the lower membrane potential necessary 
to drive V-ATPase activity (Ishida, Nayak, Mindell, \& Grabe, 2013). Here the $\mathrm{H}^{+}$leak into the cytosol may signal cytoplasmic $\mathrm{pH}$-sensitive proteins.

In addition, the acidic endosomal or phagosomal lumen may coordinate the activity of other pH-sensitive ion channels or transporters such as the lysosomal calcium channel TRPML2. TRPML2 releases $\mathrm{Ca}^{2+}$ into the cytosol by integrating the luminal $\mathrm{pH}$ of lysosomes and the second messenger nicotinic acid adenine dinucleotide (Pitt et al., 2010; Zhu, Evans, Ma, Parrington, \& Galione, 2010). Here, pH-dependent calcium efflux of TRPML2 from phagosomes may signal to $\mathrm{Ca}^{2+}$-sensitive cytoplasmic proteins to activate the recruitment of Vps34 complex II. Together, there are several means, and possible candidate channels and/or transporters that may drive a $\mathrm{pH}$ dependent ion release from endosomal and phagosomal membranes to facilitate the recruitment of Vps34 complex II machinery.

Lastly, we found that the phosphorylation pattern of UVRAG, a subunit of the Vps34 complex II, changed in response to acidification (Figure 3.9). These data suggest that UVRAG itself may be regulated by a $\mathrm{pH}$-dependent kinase that responds to changes in luminal $\mathrm{pH}$, directing Vps34 complex recruitment and activity. In fact, it was recently reported that UVRAG is phosphorylated by mTOR to increase Vps34 activity following autolysosome reformation and the authors proposed that other kinases may also act on UVRAG (Munson et al., 2015). This supports a hypothesis that a $\mathrm{pH}-$ dependent kinase that has yet been identified, or possibly mTOR itself, may coordinate UVRAG and Vps34 activity and association.

It would be interesting to investigate candidate $\mathrm{pH}$-sensitive kinases or proteins that may regulate Vps34 recruitment to endosomal membranes. In addition, it would be vital to understand if there are integral lysosomal proteins that are modified and/or act as a $\mathrm{pH}$ sensor. One way to approach this would be to isolate phagosomes under resting, acidified and neutralized conditions 
as I did in Chapter 3. Then using mass spectrometry, investigate the phosphorylation states or other post-translation modifications, of isolated proteins and see how they may be modified in response to changes in luminal $\mathrm{pH}$. Additionally, it would be interesting to employ a protein interaction assay, such as BioID, for Vps34 complex II subunits and identify candidate proteins that interact with these subunits in response to luminal $\mathrm{pH}$ changes. Together, these two experiments may help to identify candidate $\mathrm{pH}$-sensors that interact with the Vps34 complex II and that could be used as targets for downstream analysis.

\subsubsection{The tubular phagocytic cup as a model to study phagosome maturation}

As we and others have shown (Naufer et al., 2018; Prashar et al., 2013), the maturation of the tubular phagocytic cup is not limited to the recruitment of early endosomal markers. In fact, late endosomal and lysosomes fuse with and deliver their contents prior to tPC scission and do so independent of the presence of PtdIns(3)P. We suggest that the temporal and spatial resolution stemming from a long tubular phagocytic cup provides us with a robust model to resolve the molecular events of phagosome maturation more clearly. The ability for us to track PtdIns(3)P on the long tubular phagocytic cup over time enabled us to elucidate a novel $\mathrm{pH}$-dependent mechanism that controls the dynamics of this lipid. We believe that by using this model we will be able to gradually decipher the molecular events occurring during phagosome maturation that are missed during the rapid turnover of canonical spheroidal targets.

For example, it would be interesting to improve our understanding of the mechanisms that drive the changeover of Rab5 to Rab7, a necessary stage prior to early to late endosomal fusion (Nordmann et al., 2010). Our model supports the Rab5 to Rab7 transition, as active Rab7 is only recruited after Rab5 recruitment. Interestingly, preliminary work by Amriya Naufer (data not 
shown), shows that consistent with the canonical model, LAMP1 is recruited to tPCs once Rab5 and EEA1 are eliminated, however, Rab7 behaves differently. Rab7 localization to tPCs occurs as early as 5 mins following bacterial attachment, but is only activated at a significant rate following 30 minutes of phagocytosis initiation. It would be interesting to understand the requirements for Rab7 activation using our tPC model, as the canonical model suggests that Rab7 is recruited to the maturing phagosome, where Mon1/Ccz functions as a Rab7 GEF and activator (Nordmann et al., 2010). Interestingly, the mechanism of Mon1/Ccz activation remains elusive but its recruitment is Rab5-dependent. Thus, using the tPC's increased spatiotemporal resolution may help to elucidate the mechanism by which inactive Rab7 is recruited to phagosomal membranes and is turned on through active Mon1/Ccz. It would be interesting to investigate if PtdIns(3)P removal and luminal acidification is a prerequisite for Mon1/Ccz GEF activity, stimulating Rab7 activation. To investigate one could utilize the truncated form of RILP, RILP-c33, a known Rab7 effector that interacts with GTP-bound Rab7 (Cantalupo, Alifano, Roberti, Bruni, \& Bucci, 2001). Using this construct, we could investigate the timing of Rab7 activation during tubular phagocytic cup maturation and the interplay between PtdIns(3)P levels and phagosomal luminal acidification.

In addition to understanding the events of phagosome maturation, it would be useful to use tPCs as a model to study the role of endo-lysosomal markers in immunity and cellular homeostasis pathways. For example, PtdIns(3)P plays roles in several pathways that regulate immune responses. In the context of immunity, PtdIns(3)P may regulate overproduction of reactive oxygen species (ROS) on growing tPCs to help facilitate the antimicrobial response of macrophages. In fact, the p40phox subunit of NADPH oxidase is required for oxidase activation, thus excess PtdIns(3)P may lead to an over-activation of the complex and promote antimicrobial activity of macrophages (Tian et al., 2008). ROS over-production has been linked to pathological situations 
in chronic diseases leading to redox damage (Brieger, Schiavone, Miller, \& Krause, 2012). Furthermore, PtdIns(3)P has also been linked to autophagy induction by coordinating signalling complex formation and facilitating the biogenesis of omegasomes (Burman \& Ktistakis, 2010; Nascimbeni, Codogno, \& Morel, 2017), thus its retention on tPCs may imply a role for autophagy in this process. Overall, PtdIns(3)Ps evidently play important roles for many cellular events that support the antimicrobial and degradative functions of macrophages. Therefore, the persistence of this lipid on tPCs may have implications beyond phagocytosis that would be interesting to investigate using the spatiotemporal resolution imparted on tPCs to resolve the machineries and events that coordinate these processes. 


\subsection{Lysosome remodelling in activated phagocytes}

Phagocytes like macrophages and dendritic cells are highly plastic, adapting to an array of internal and external cues to exert inflammatory and anti-inflammatory responses. These immune cells must strategically adapt their cellular state and function in response to different environmental factors. Research has mainly focused on the ability of these cells to alter their metabolic state, cytokine and chemokine profiles and microbicidal agents (Janssens et al., 2014; Kelly \& O’Neill, 2015; Porta et al., 2015; Trombetta et al., 2003), whereas, little attention has been given to how they adapt organelles in response. Research has shown that macrophages and dendritic cells are able to reorganize their endomembrane system in response to immune stimulation with factors like lipopolysaccharides (reviewed in; Hipolito et al., 2018). In fact, literature reports that dendritic cells downregulate their proteolytic activity to help preserve antigens and that endomembrane organelles can transform from punctate structures to a tubular network. Lysosome tubulation has been linked to increased pinocytic uptake, exchange of antigen content and MHCII-peptide presentation. Although, the mechanisms that control these events are not well understood.

In Chapter 4, I present data examining a novel mechanism underlying the reorganization of the endo-lysosomal system in activated immune cells. First, we discovered that lysosomal volume and retention capacity is rapidly amplified in LPS-activated phagocytes, which relies on de novo protein synthesis via the mTORC1-S6K-4E-BP1 axes and is independent of transcriptional mechanisms. Interestingly, we showed that LPS-driven lysosomal expansion depends on mTORC1-dependent selective and enhanced translation of lysosomal proteinencoding mRNAs. Ultimately, we present evidence that mTORC1- and translation-dependent increase in lysosome size and holding capacity is critical for efficient and rapid antigen presentation by dendritic cells. Additionally, in Chapter 5, I present data identifying a role for 
lysosome remodelling and adaptation following adjuvant stimulation, revealing potential applicability of this phenomenon in vaccine development.

\subsubsection{Acute phagocyte activation bolsters lysosomal volume and holding capacity}

Functionally, macrophages and dendritic cells are efficient at macropinocytosis and phagocytosis, whereby they take-up material and internalize them for degradation and/or antigen processing and presentation. We showed that phagocytes activated with LPS remodel their lysosome system into an expanded tubular network and increase holding capacity. To this end, using fixed-cell, live-cell and super-resolution microscopy we report that phagocytes with pre-labeled lysosomes increase lysosomal volume after LPS exposure (Figure 4.1 \& Figure 4.2). This increase in volume was accompanied with an enhancement in lysosome biogenesis (Figure 4.5 \& Figure A4). Moreover, we demonstrate that phagocytes stimulated with LPS rapidly increased their endocytic capacity compared to resting cells, whereas, they do not differ in recycling - therefore augmenting retention capacity (Figure $4.3 \&$ Figure 4.4). Together these data support a theory that lysosomes are remodelled to boost holding capacity in phagocytes following LPS exposure. The data is consistent with work by Swanson et al. completed in the 1980s, showing that phorbol ester-activated macrophages retain fluid-phase material more effectively than resting macrophages (J. A. Swanson et al., 1985; J Swanson et al., 1987). We found that increased volume was dependent on de novo protein synthesis, as treatment with cycloheximide blunted lysosome biogenesis and volume. Overall, activated phagocytes not only remodel their lysosomes into a tubular network, but also expand this organelle.

Lysosomal expansion may play a regulatory role in boosting a phagocyte's inflammatory response as the capacity to engulf and process large volumes of material is one of the main characteristics of 'professional' phagocytes. In fact, it has been reported that macrophages and 
dendritic cells can engulf synthetic particles up to 30 microns in diameter, in addition, they can internalize 10-30 IgG-opsonized beads (Zent \& Elliott, 2017). Evidently, their capacity to internalize excess material must be balanced by cellular remodelling, which may be limited by the amount of available membrane. Depending on the target, macrophages adapt and increase their surface area from 20-500\% (G J Cannon \& Swanson, 1992; Lam, Herant, Dembo, \& Heinrich, 2009), which is thought to be driven by increased focal exocytosis (Masters, Pontes, Viasnoff, Li, \& Gauthier, 2013; Zent \& Elliott, 2017). Macrophages do enhance plasma membrane remodelling to balance the phagocytic demand, however, increased lysosomal holding capacity may indeed play a vital role here, where activation of phagocytes may boost lysosome biogenesis to bolster holding capacity following uptake. Moreover, it has been shown that prior exposure of bonemarrow derived macrophages to apoptotic cells can result in a prophagocytic 'priming' effect enhancing phagocytic capacity by nearly 60\% (A-Gonzalez et al., 2009; Mukundan et al., 2009), characterized by increased plasma membrane remodelling and synthesis; possibly lysosome biogenesis is being enhanced to balance the increase in holding capacity. Similarly, immune cells primed with bacterial factors whose lysosomes are remodelled may be better suited to fight a persistent infection. In fact, it would be interesting to test this, by pre-activating phagocytes with LPS and then introduce remodelled cells to bacteria and measure their bacterial killing capacity and/or ability to process them through antigen presentation.

However, mature dendritic cells are reported to have reduced endocytosis (Barois et al., 2002; Garrett et al., 2000), we showed that dendritic cells still exhibit extensive pinocytosis for at least 8 hours post-activation, providing ample time for cells to internalize and accumulate antigenic material. This data is also consistent with reports showing that DCs are still able to internalize significant amounts of extracellular content (Drutman \& Trombetta, 2010; Platt et al., 2010). 
Consistent with this concept, we showed here that LPS stimulation of BMDCs significantly increased presentation of two distinct antigenic peptides, HEL and $\mathrm{E}_{\alpha}$, peptides as early as 4-6 $\mathrm{h}$ of antigen uptake (Figure 4.12). Importantly, antigen presentation was highly dependent on the activities of mTOR and S6K, suggesting that up-regulated translation coupled to lysosome expansion helps drive antigen presentation efficiency. Additional mechanisms may also assist antigen presentation in BMDCs including LPS-mediated alteration of lysosome luminal properties like $\mathrm{pH}$ and exocytosis of lysosome tubules, which also depends on mTOR (Marianne Boes et al., 2002; Chow et al., 2002; Saric et al., 2016).

The extent to which lysosome tubulation, expansion, secretion and luminal remodelling contribute to antigen presentation will need to be assessed in future studies. For example, it would be interesting to investigate the impact of the luminal properties of the tubular network on antigen presentation. Are lysosomal tubules more acidic or less acidic than punctate lysosomes in activated cells? I provide preliminary data for this in section 6.2.4. To investigate the role lysosome tubulation plays on antigen presentation may be difficult, as we have not yet identified a lysosomespecific mediator of tubulation. Many of the inhibitors or protein targets we know that coordinate lysosome tubulation (i.e. Torin1, microtubule depolymerizing agents etc.) play important roles in other pathways that may confound the analysis. To that end, it would be ideal to identify a lysosome tubule mediator first, such as a lysosomal tethering protein that coordinates lysosome coalescence or the role fission machinery may play upon LPS-activation.

Organelle tubulation is not reserved to lysosomes, as endoplasmic reticula and mitochondria form vast tubular networks (Schwarz \& Blower, 2016; C. Wang et al., 2015). In fact, it is well known that the mitochondrial tubulation is coordinated by fusion and fission machinery in response to the cell's energy levels (Rambold, Kostelecky, Elia, \& Lippincott-Schwartz, 2011; 
Wang et al., 2015). Interestingly, starvation-induced mitochondrial elongation is mediated by phosphorylation-induced down-regulation of dynamin-related protein 1 (Drp1), a regulator of dynamin fission machinery, leading to unopposed mitochondrial fusion and tubulation (Feng \& Kornmann, 2018; Morita et al., 2017). An alternatively spliced DRP1 isoform, DRP1ABCD, was found to localize onto lysosomes, late endosomes and the plasma membrane (K. Itoh et al., 2018). Whether DRP1ABCD is active in phagocytes is unknown, nevertheless, if confirmed, it would be interesting to investigate if LPS exposure down regulates DRP1ABCD activity, blocking fission machinery and driving lysosome tubule formation. Moreover, another candidate protein that would be interesting to investigate is Spinster, a putative lysosomal efflux permease, that is needed for ALR tubule formation and has also been reported to coordinate lysosome tubule network formation in Drosophila muscle cells (Johnson et al., 2015; Rong et al., 2011). Investigating the potential role these proteins may play in tubule formation would add to our understanding of the machinery that controls lysosome tubulation and will provide us with a model to study this phenomenon more explicitly in immune cells.

\subsection{2 mTOR-S6K-4E-BP axes control lysosome expansion}

Our work shows that phagocyte activation leads to a rapid expansion of lysosome volume within two hours of LPS exposure. This expansion is driven by de novo protein synthesis as indicated by the cycloheximide-mediated block in lysosome expansion. Surprisingly, our data also suggests that rapid expansion of lysosome volume and holding capacity in response to LPS stimulation, is not dependent on transcriptional changes to lysosome protein-encoding genes, since the corresponding mRNA levels did not increase, even after $6 \mathrm{~h}$ post-LPS activation (Figure 4.6). Consistent with this, the timing of TFEB and TFE3 translocation into the nucleus did not parallel 
initial lysosome enlargement and was consistent with previously reported kinetics of LPS-induced TFEB and TFEE3 nuclear translocation (Pastore et al., 2016), and their deletion did not impair tubulation or rapid lysosome expansion. In contrast to transcriptional regulation, we observed that mTOR-dependent translational mechanisms play a key role in LPS-mediated lysosome expansion (Figure 4.7, Figure 4.8 \& Figure 4.9). First, LPS activates mTORC1, as indicated by increased phosphorylation of S6K and 4E-BP1, and enhanced protein synthesis, whereas its inhibition abrogated lysosome tubulation and expansion. Second, while translation machinery is governed by a plethora of mechanisms, including mTOR-independent pathways, our data reveal that S6Ks and 4E-BPs play a major role in governing lysosome size in response to stress (Figure $4.10 \&$ Figure 4.11). Altogether, we provide a novel mechanism by which phagocytes respond to activation by remodelling the lysosome population by scaling up lysosome biogenesis through mTOR-dependent translational control, independent of transcription. Additionally, we present data illustrating that the S6K-mTOR signalling axis regulates antigen presentation in APCs likely through lysosome remodelling.

Nonetheless, the mechanism by which mTOR controls lysosome-specific mRNA translation is not fully understood. Potentially, mTORC1-mediated inhibition of 4E-BPs releases the translation brake imposed on the translation initiation factor eIF4E (Roux \& Topisirovic, 2018; Thoreen et al., 2012). The role of S6Ks in this process is potentially more complex given its numerous targets that modulate translation (Figure 1.3). Active ribosomal S6K regulates ribosomal protein rpS6 increasing ribosomal activity and translation efficiency, the translation initiator factor eIF4B promoting translation initiation, PDCD4 promoting the helicase function of eIF4A, eEF2K which governs elongation rates, and SKAR that may promote mRNA splicing and maturation (Ma, Yoon, Richardson, Jülich, \& Blenis, 2008; Nandagopal \& Roux, 2015; Roux \& Topisirovic, 2018). 
Altogether, mTORC1's regulation of 4E-BP1 and S6K activity may broadly promote efficient mRNA translation driving lysosomal remodelling. To provide a mechanistic insight into how 4EBPs and S6Ks coordinate lysosome remodelling and antigen presentation, it would be beneficial to use genetic models. Using a mouse strain double deleted for S6K1 and S6K2, and the 4EBP1 ${ }^{\text {Ala }}$ transgenic mice $\left(4 \mathrm{EBP} 1^{\mathrm{M}}\right)$ which uses a tetracycline inducible system (Hsieh et al., 2010), we can investigate whether 4E-BP1 and S6K directly regulate lysosome remodelling in primary BMDMs and BMDCs. Using these primary BMDCs and BMDMs generated from these mice models, we can target downstream proteins of S6K using pharmacological and genetic approaches to further elucidate the mechanism.

We do suspect that mTORC1-regulated mRNA translation is not reserved to only lysosomal mRNAs and is a mechanism that broadly serves to rapidly scale the activity and size of other organelles in response to metabolic alterations and stress. Consistent with this idea, inhibition of TSC1/2, an inhibitor of mTORC1, or overexpression of S6Ks increased the size and length of cilia in Chlamydomonas reinhardtii and zebrafish (Yuan et al., 2012). Future work should focus on investigating the impact phagocyte activation has on the translation of mRNA targets that control the function of other organelles. To this end, it would be interesting to perform global RNA- sequencing (RNA-seq) on pooled or individual heavy-polysomal fractions to investigate whether subsets of organelle mRNAs are hyper-translated following LPS in an mTOR-dependent manner. For example, we may find an upregulation of mRNAs that control the endoplasmic reticulum's UPR pathway, to compensate for the boost in protein synthesis and folding, paralleling with this pathway's activation following LPS stimulation (Janssens et al., 2014).

Moreover, this screen may reveal additional lysosomal mRNA targets that we did not initially probe for. Indeed, the cell may scale subsets of lysosomal mRNAs depending on the signal 
context, cellular state and activation timing. For example, it may be beneficial to quickly scale lysosomal membrane protein levels in order to expand the lysosomal membrane through translation mechanisms. Whereas, delayed transcriptional mechanisms may regulate lysosomal functional proteins such as V-ATPase subunits and cathepsin protein levels. Moreover, investigating the role of membrane remodelling in this process would be interesting. Perhaps, mTOR-dependent translation increases the levels of lipid biogenesis mediators, driving lysosomal membrane biogenesis, or membrane influx in order to support the increase in lysosome volume and membrane extension. As previously discussed, global RNA-seq analysis of polysomal fractions may elucidate the role of target lipid biogenesis enzymes.

Our polysome and protein data show that LPS activation selectively activates the translation of a subset of lysosomal mRNAs. How can mTORC1 selectively target the translation of a pool of mRNAs? It is well accepted that in addition to its effects on global protein synthesis, mTOR selectively increases the translation of specific mRNA subsets. Of these, the best characterized mRNAs are those with a $5^{\prime}$ terminal oligopyrimidine sequence (5'TOP), which renders its translation mTOR-sensitive (Gaard et al., 2008; Hsieh et al., 2012; Meyuhas, 2000; Thoreen et al., 2012). The most studied 5'TOP mRNAs are those that encode for translation and ribosomal protein machinery and respond to changes in cellular energy levels and state in an mTOR-dependent manner, whereas, other genes tend to be overlooked. Interestingly, in a human cell-line, it was reported that mRNAs encoding for proteins other than translation machinery also contain 5 'TOPs, in fact, they found that $2 \%$ of the 5'TOP candidate genes encoded for lysosomal proteins (Yamashita et al., 2008). However, based on our searches the murine lysosomal mRNAs encoding LAMP1 and V-ATPase subunits that were probed for do not contain the classical 5'TOP sequences. 
It is possible that lysosomal mRNA translational regulations might have evolutionary diverged (Odom et al., 2007), whereby murine and human mTOR-sensitive mRNA sequences differ. In fact, it was determined that a significant number of mTOR-sensitive mRNAs lack the 5'TOP motif and contain other structural markers such as elongated unstructured 5'UTRs or 3'UTRs that regulate translation (Bilanges et al., 2007; Gandin et al., 2016; Larsson et al., 2012). Future work should investigate whether the 5' and/or 3' UTRs of our lysosomal targets are sensitive to mTOR regulation in response to LPS. To test this, we could design a reporter screen by cloning candidate UTRs into luciferase reporter plasmids and measure luciferase levels in response to LPS and mTOR inhibition (Campos-Melo, Droppelmann, Volkening, \& Strong, 2014).

We suspect that transcripts that encode for lysosomal proteins may be a useful functional model to identify novel mRNA sequence or structural motifs that prompt mTOR-dependent translation. Additionally, this information may shed light onto the role of mTOR-dependent mRNA translation in other cells-types, including Drosophila muscle cells and exocrine acinar cells, where the occurrence of lysosomal tubulation is less understood (Bendayan \& Gisiger, 2001; A. E. Johnson et al., 2015). Identifying novel pools of mTOR-sensitive mRNAs may provide useful insight into how organelle function and protein pathways are scaled in response to stress in other cell-types.

\subsubsection{Lysosome remodelling during vaccine response}

Our data suggest that lysosomal remodelling plays a functional role in LPS-activated phagocytes, boosting antigen presentation and T-cell priming. Within the vaccine discovery sector, there is great interest in natural and synthetic toll-like receptor agonists as adjuvants, as they strongly induce antibody titer and quality, immune memory, type of immunity, and can reduce the antigen 
dose within a vaccine needed to develop immunity (Gutjahr et al., 2016). In fact, many adjuvants bind to and activate the same signalling pathways of natural adjuvants. For example, monophosphoryl-lipid A, the adjuvant used in common HPV vaccines, signals through the tolllike receptor 4 pathway, as does LPS. We therefore speculated whether lysosomal remodelling in APCs may play an important role in the immunostimulatory effects of adjuvant molecules commonly used today.

Our preliminary data determined that two of the tested adjuvant compounds induced lysosome tubulation and volume increase, whereas two others did not (Figure 5.1). Thus, identifying two clinically relevant compounds that induce lysosome remodelling. Indeed, both activated mTOR signalling, as measured by S6K phosphorylation, and promoted antigen presentation, as determined by increased MHCII-peptide complex formation in APCs and T-cell dependent IL-2 secretion (Figure 5.2Figure 5.3, Figure 5.5). Moreover, lysosomal remodelling and antigen presentation relied on mTOR, and likely S6K activity as their inhibition abrogated antigen presentation and T-cell priming (Figure 5.5). Interestingly, adjuvant mixing augmented compound B-induced antigen presentation and $\mathrm{T}$-cell activation, corresponding with clinically relevant adjuvant complex systems such AS04 used in HPV vaccines (Garçon et al., 2011).

Our data provides novel evidence that lysosomal tubulation and remodelling is not only reserved to Toll-like receptor signalling, as one of our compounds is a NOD receptor-agonist. Suggesting that the mTOR-S6K signalling pathway may serve as a convergence point for multiple inflammatory pathways that promote phagocyte activation through lysosome remodelling and increased antigen presentation. In the future, our lysosome tubulation and holding capacity assays may be used as a model to test for the potency and efficacy of candidate adjuvants. We suspect that adjuvants that prompt lysosome remodelling in APCs are good candidates for downstream 
analysis. If we could design a high-throughput approach to measure lysosomal tubulation efficiency, such as using high-content fluorescent scanners coupled with automated algorithms that can analyze lysosomal tubule index, it would be feasible to apply this on a large-scale format capable of testing several targets.

Future studies should focus on uncovering the signalling pathways that coordinate lysosome remodelling following adjuvant stimulation. Whether they signal through the same LPSmTOR-S6K axis remains an enigma. By using the $\mathrm{S} 6 \mathrm{~K}$ and $4 \mathrm{EBP} 1^{\mathrm{M}}$ genetic mouse models previously described, in combination with polysome profiling assays, we can elucidate the signalling pathways activated following adjuvant activation. Additionally, it would be important to dissect how lysosome remodelling bolsters antigen presentation in activated-APCs. Is the rapid remodelling necessary for all or some of the following stages: i) antigen uptake ii) antigen processing iii) antigen-MHCII complex formation and iv) trafficking of antigen-MHCII complexes to the plasma membrane. Altogether, we present novel data linking lysosome remodelling in adjuvant-activated APCs bolstering antigen prestation, likely promoting immunity during vaccination.

\subsubsection{Biochemical properties and function of lysosomal tubulation}

Our data supports a hypothesis that lysosomal reorganization increases the retention capacity of cargo and facilitates antigen presentation in activated cells. Why else would tubulating the lysosomal lumen be advantageous to the cell? A plausible physiological function for immune cells is to create an inter-connected network of organelles with a homogenized lumen. Luminal homogenization may be beneficial to antigen presenting cells to enhance cargo exchange and the sampling of antigenic material during antigen processing and presentation. Due to the high 
polymorphisms in the MHCII peptide-binding groove, MIIC compartments may hold different MHCII molecules, giving rise to lysosomal heterogeneity in terms of the antigen molecule that can be recognized. Forming an inter-connected network and homogenizing the lumen may statistically increase the probability of antigen-MHCII combinations by allowing for free diffusion of antigens through the lumen, and free lateral diffusion of MHCII on the lysosomal membrane. Moreover, the subcellular distribution of lysosomes impacts lysosomal $\mathrm{pH}$ and proteolytic activity, where peripheral lysosomes are less acidic then perinuclear lysosomes (Johnson et al., 2016). Homogenization of the lysosomal lumen may increase the overall degradative capacity of the lysosomal population by liberty of access to proteolytic enzymes and V-ATPases to acidify the lumen.

It would be interesting to test the homogenization theory and determine its potential impact on antigen processing and presentation. To test this, we could use photoactivatable probes for the lysosomal lumen and membrane, such as a photoactivatable GFP-LAMP1 (Michael Davidson; Addgene plasmid \# 57143) and measure their distribution throughout the lysosomal network upon activation. Following selective activation, one would measure the spread of the fluorescent signal across pre-labeled lysosomes over time. We would expect to see faster diffusion in activated cells, if tubular lysosomes form an inter-connected network, compared to the diffusion in resting cells with punctate lysosomes.

Furthermore, lysosomal tubulation may facilitate the trafficking of internalized content. As we have previously reported, lysosomal tubules are more motile than punctate lysosomes in activated cells (Mrakovic et al., 2012) and lysosomal tubulation increases MHCII-antigen complex delivery to the plasma membrane (Saric et al., 2016; Vyas et al., 2007). Thus, we speculated whether lysosomal tubules acquire internalized material more quickly than punctate lysosomes to 
promote cargo uptake and exchange. I pursued this and tested whether punctate and tubular lysosomes acquire incoming cargo at different rates, within LPS-activated RAW macrophages. To test this, I pre-labeled lysosomes using our standard pulse-chase protocol with Alexa-546 dextran and then induced lysosomal tubulation (Figure 6.3a). Then, I exposed tubulating cells to a secondary fluid-phase dye (Alexa-488 dextran) for 10 minutes to allow internalization and followed its accumulation into pre-labeled tubules and puncta. Using Pearson's correlation coefficient we quantified the degree of colocalization and found that there was no difference between the rate at which tubules and puncta acquired the second probe over time (Figure 6.3a). Our preliminary data therefore suggests that tubulation may not function to enhance the acquisition of incoming cargo, although it would be interesting to do the same with receptor-mediated cargo, such as aggregated IgG.

Additionally, lysosomal membrane extension may impact luminal properties, giving rise to lysosomes of differential function that may bolster phagocyte function. In fact, the subcellular distribution and localization of lysosomes alters lysosomal luminal $\mathrm{pH}$ and proteolytic activity (Johnson et al., 2016). Moreover, the extension of spheroidal lysosomes into tubular structures may impact luminal ion levels and concentrations, which could translate into changes in electrochemical gradients and impact the function of transporters, channels and luminal proteins. Alterations in the luminal environment may affect the activity of cysteine cathepsins, the recruitment of signalling complexes onto the lysosomal membrane, as well as, coordinate the recruitment of motor proteins necessary for lysosomal motility (Li et al., 2016; Pu et al., 2016; Zoncu, Bar-Peled, et al., 2011). Indeed, both lysosomal calcium and $\mathrm{pH}$ are important for phagosome maturation, lysosome motility and structure, and bacterial clearance (Dayam, Saric, Shilliday, \& Botelho, 2015; Li et al., 2016). Therefore, we briefly investigated whether luminal 
calcium and $\mathrm{pH}$ differed between tubules and puncta within activated cells and performed preliminary experiments. We used a fluid-phase calcium-sensitive fluorescent probe, Calcium green-1 dextran, to compare calcium in punctate and tubular lysosomes of activated BMDMs (Figure 6.4a) and found that relative calcium levels were similar between the two lysosome pools. Yet, it is possible that the activity of calcium channels in tubular and punctate lysosomes may differ, giving rise to differential signalling and effector protein recruitment (X. Li et al., 2016).

Interestingly, when we used pHRodo-dextran, a pH-sensitive fluid-phase marker whose fluorescence increases with decreasing $\mathrm{pH}$, we found that tubular lysosomes appeared less fluorescent than punctate lysosomes in activated BMDMs (Figure 6.4b). Surprisingly, this data suggests that tubular lysosomes may be less acidic than puncta. We attempted to measure the $\mathrm{pH}$ of tubular lysosomes by calibrating lysosomal $\mathrm{pH}$ with known $\mathrm{pH}$ buffers and ionophores, however, addition of ionophores caused tubules to collapse (data not shown), suggesting that luminal $\mathrm{pH}$ and/or $\mathrm{pH}$ gradients may coordinate lysosomal tubule formation. In addition, we also acquired live-cell confocal videos of pH-Rodo labeled lysosomes in activated BMDMs and observed an interesting phenotype, where bright $\mathrm{pH}$-Rodo labeled puncta localized to the tips of growing tubules (Figure $6.4 \mathrm{c}$ ). Together the preliminary data suggest that $\mathrm{pH}$ may play a role in lysosome tubule formation and that tubules may differ from punctate lysosomes in function and luminal properties. Future work should focus on determining how lysosomal $\mathrm{pH}$ may differ in the two pools, such as investigating V-ATPase complex recruitment and activity within the two lysosomal pools.

The degradative capacity of lysosomes is well understood to be controlled by $\mathrm{pH}$-sensitive proteases. Given the differences in luminal $\mathrm{pH}$ we observed, we wondered whether tubular and punctate lysosomes are functionally distinct in activated cells. We measured the proteolytic 
activity of both pools using a fluid-phase protease-sensitive protein, DQ-BSA (ThermoFisher Scientific). The DQ-BSA protein is heavily conjugated with self-quenching BODIPY dyes and when in the presence of hydrolytic enzymes, it is cleaved into smaller peptides, relieving the quenching effect (Figure 6.5a). We measured the fluorescence of DQ-BSA in tubules and punctate lysosomes in activated RAW cells, BMDMs and BMDCs and determined that tubules in macrophages appear to be more degradative than puncta, whereas, they do not differ in dendritic cells (Figure 6.5b). This cell-type dependent response is interesting, as it follows previous reports of APCs downregulating proteolysis to retain peptides for antigen presentation, whereas lysosomes in macrophages are 50-times more functionally degradative to readily clear infection (Delamarre et al., 2005).

Nevertheless, we did measure a relative increase in lysosomal $\mathrm{pH}$ in BMDM tubular lysosomes compared to puncta, seemingly contradicting the functional output of tubules in BMDMs. Therefore, future studies should measure the $\mathrm{pH}$ of the lysosomal pools in both BMDMs and BMDCs. One may be interested in investigating how luminal $\mathrm{pH}$ in tubules and puncta regulate lysosomal cathepsin activity by co-staining lysosomes with pHRodo-dextran, distinguishing punctate and tubular lysosomes, with cathepsin-specific Magic Red protease assays (ImmunoChemistry Technologies) and measure if there are differences in activity. Moreover, it would be fascinating if we could separate tubular and punctate lysosomes following standard lysosome membrane fractionation, then determine the amount of and maturation state of cathepsin targets within the two pools.

Tubulation and remodelling of the lysosomes may affect several of lysosome-related biochemical and functional properties in LPS-activated phagocytes. Extension of the membrane may impact luminal properties and therefore the function of resident proteins, as well as, increase 
the amount of membrane surface area available for signalling complex recruitment onto the lysosomal membrane. Remarkably, this may promote and increase mTORC1 complex recruitment and localization to the lysosomal membrane, whereby its activated by the Rheb GTPase, which in turn is also controlled by the lysosomal lumen. In fact, phagocyte activation may promote lysosome remodelling to increase the number and activation-state of mTORC1 complexes to enhance mTORC1-dependent activation of proinflammatory signals and possibly mTORC1dependent translation of other proinflammatory mRNA. 
6.3 Figures 
Figure 6.1

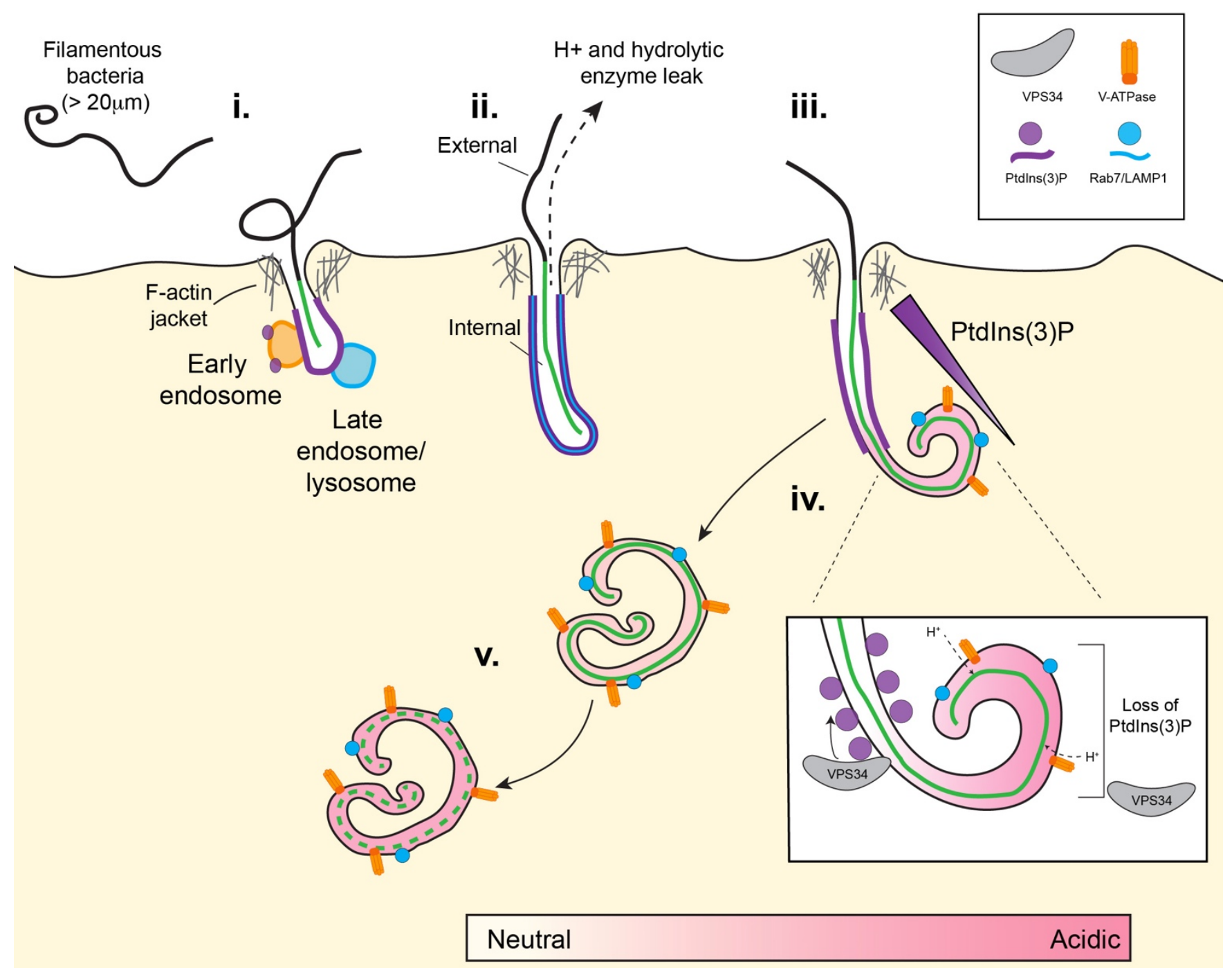


Figure 6.1: Schematic illustrating stages of tubular phagocytic cup maturation and PtdIns(3)P dynamics.

(i) Phagocytosis of filamentous bacterial targets proceeds through a tPC, which is enriched in Factin near the plasma membrane. The bases of tPCs lose F-actin and undergo maturation event, through fusion with endosomes and lysosomes. PtdIns(3)P (purple) is acquired on phagocytic cups in this way. (ii) Despite persistence of PtdIns(3)P, unsealed cups recruit the late endosomal markers, Rab7 and LAMP1 (blue). During internalization the cup remains open, causing the leakage of protons and low molecular weight hydrolytic enzymes, creating a relatively neutral structure. (ii) As filaments greater than 20 micrometers are internalized, tPCs display inverted gradients for PtdIns(3)P recruitment and acidification. The regions of tPCs close to the plasma membrane recruits PtdInsP(3)P and is relatively neutral. As the coiled regions of PCs begins to acidify, Vps34 falls off between a pH of 6.1-6.3 and PtdIns(3)P synthesis is terminated. (iv) Eventually, tPCs seal into tubular phagosomes whose lumen acidifies promoting its hydrolytic capacity. (v) The resultant antimicrobial environment allows for the complete degradation of the target. (internal bacteria: green, external bacteria: black). 
Figure 6.2

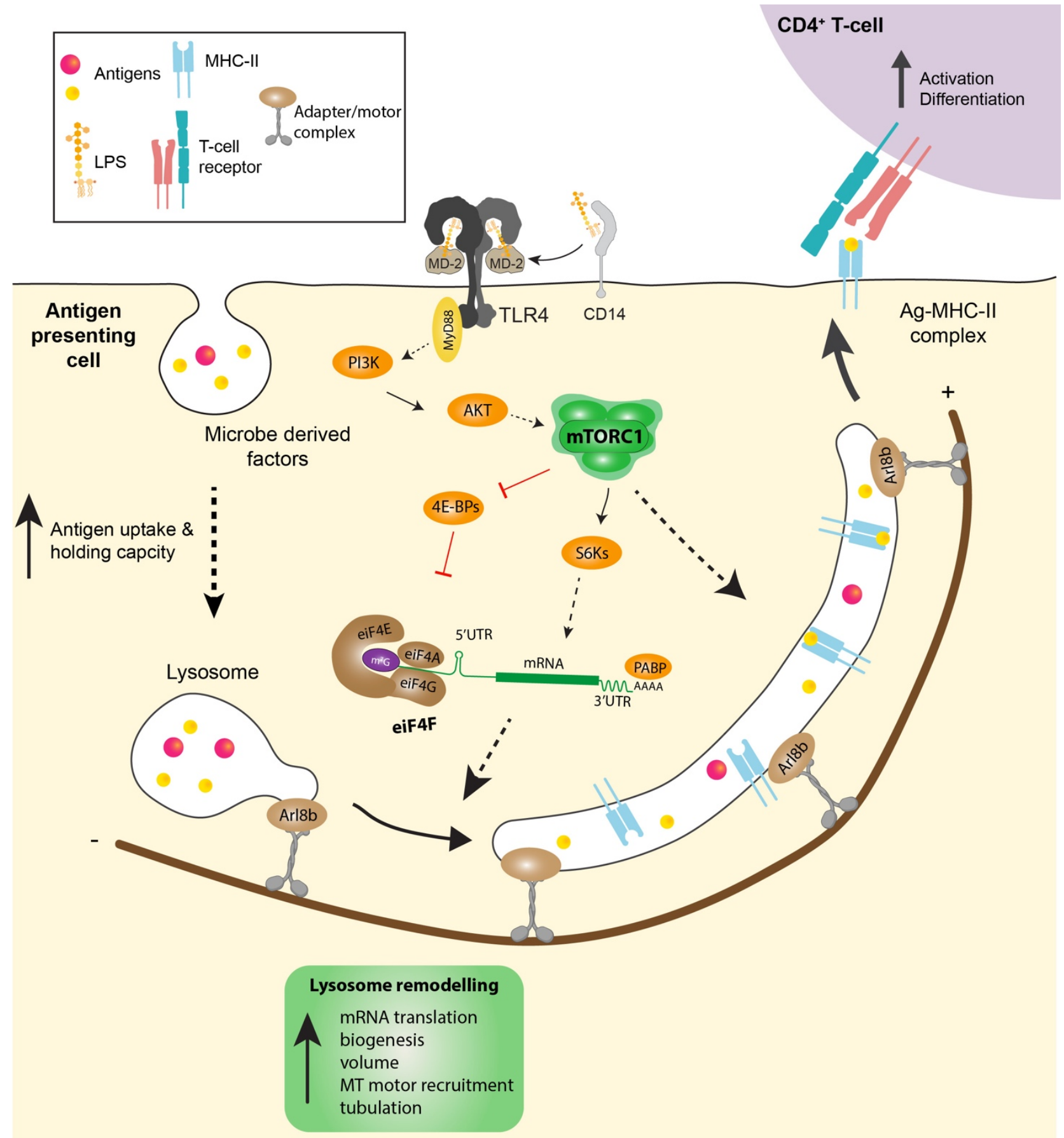


Figure 6.2: Schematic illustrating signalling pathways and molecular mediators that control lysosome remodelling in activated phagocytes.

LPS engages the PI3K-AKT-mTOR signal axis downstream of TLR4. mTOR activity stimulates lysosome remodelling by activating S6K and inhibiting 4E-BP1 activities. Together, this leads to enhanced and selective translation of lysosomal mRNA targets, boosting lysosomal biogenesis and expansion. mTOR activity augments Arl8b GTPase levels on the lysosome membrane, which likely boosts kinesin-1 recruitment to coordinate lysosome extension (Hipolito et al., 2018; Saric et al., 2016). Lysosomal remodelling boosts antigen uptake and processing to promote MHC-II antigen $(\mathrm{Ag})$ complex presentation to the plasma membrane and $\mathrm{CD}^{+} \mathrm{T}$-cell activation to bolster immunity. Solid arrows indicate established interactions or outcomes, while dashed arrows indicate indirect or unclear mechanisms. 
Figure 6.3

a

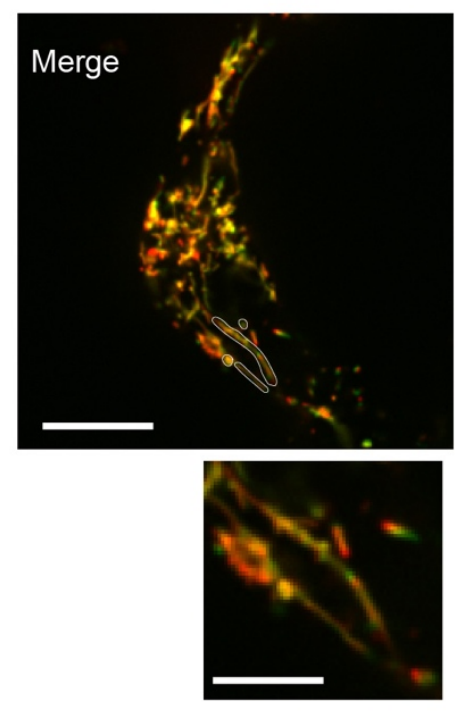

b
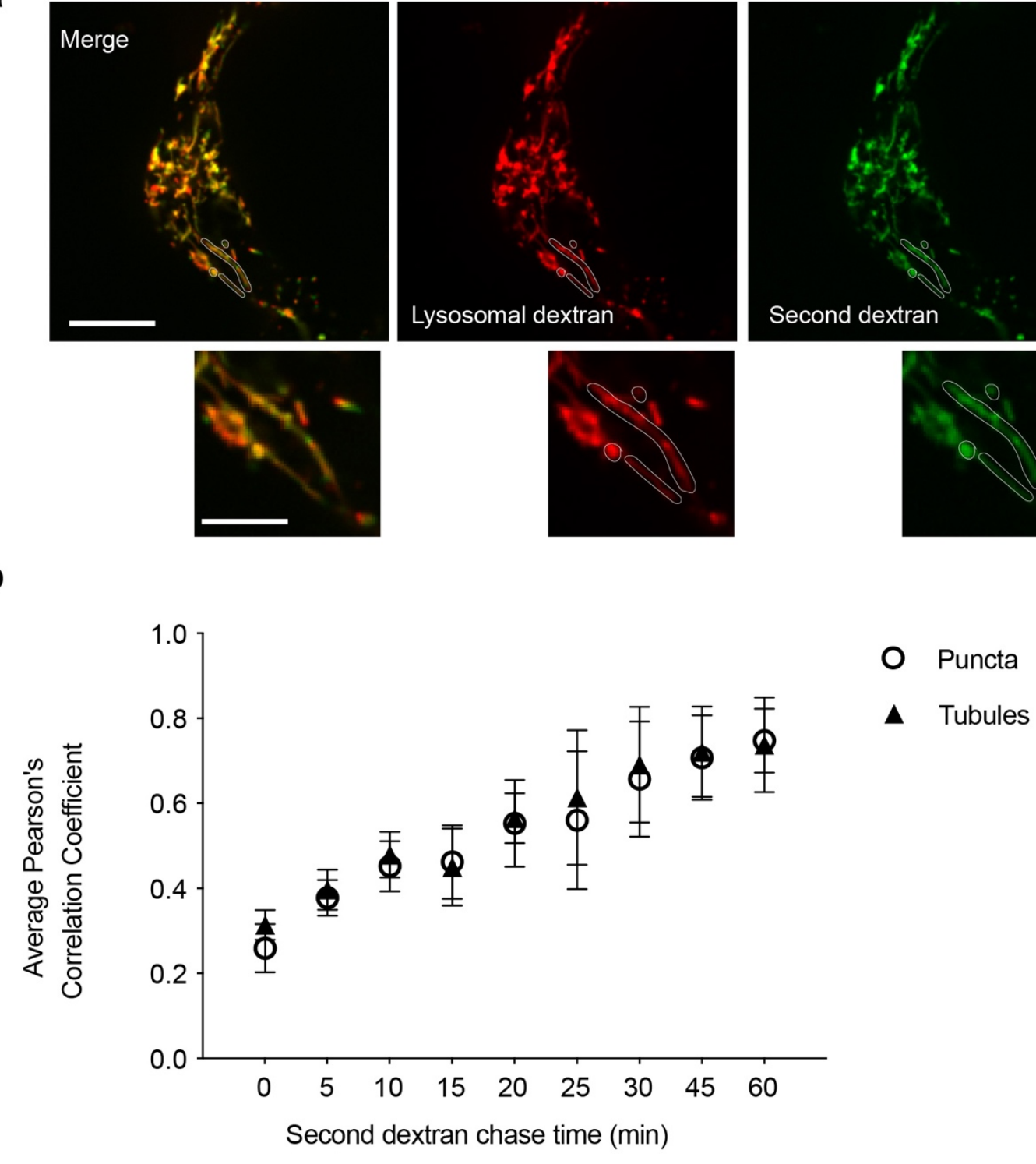

Second dextran
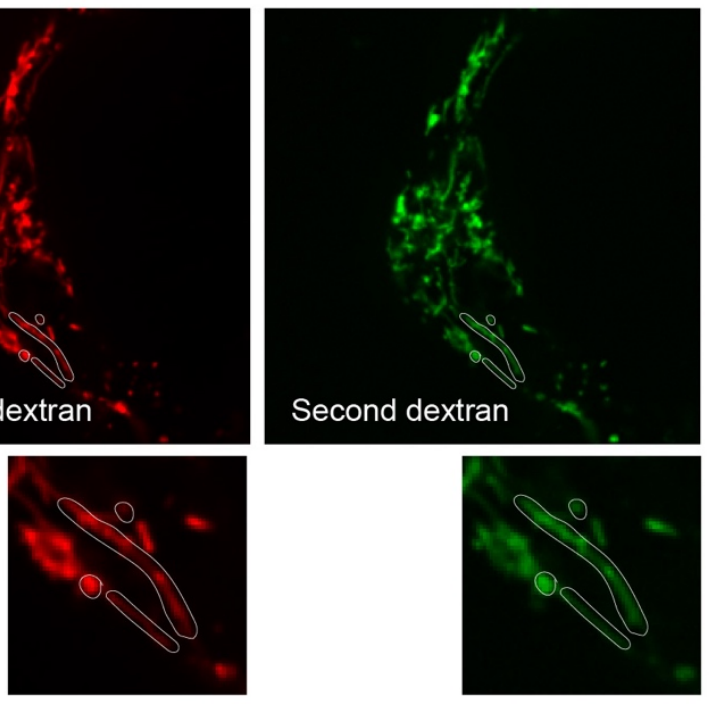

Second dextran (30 min chase) 
Figure 6.3: Fluid-phase cargo traffic to tubular and punctate lysosomes at the same rate.

(a) Method for measuring cargo trafficking to punctate and tubular lysosomes in RAW macrophages. RAW macrophage lysosomes were prelabelled with $75 \mu \mathrm{g} / \mathrm{mL}$ of Alexa-546 dextran (30 min pule, $1 \mathrm{~h}$ chase), then activated with LPS for 2 hours to induce tubulation. Next, cells were pulsed with $150 \mu \mathrm{g} / \mathrm{mL}$ of Alexa-488 dextran for $10 \mathrm{~min}$, and then chased in dextran-free media for the indicated time points. Sample micrograph shows 30 min chase time. Individual tubular or punctate lysosomes were identified using Alexa-546 as a marker and outlined. Using Pearson's correlation coefficient, we measured the degree of Alexa-488 signal in Alexa-546 pre-labeled structures. (b) Quantification using approach in a from three independent experiments. At least 10 tubules and puncta were measured across 20 cells for each experiment. Data are represented as an average Pearson's correlation coefficient for each time point. ANOVA analysis was performed, and no significant difference was found between tubules and puncta for the time points measured. Scale bar $=10 \mu \mathrm{m}$ for large micrographs and $5 \mu \mathrm{m}$ for magnified insets. 
Figure 6.4
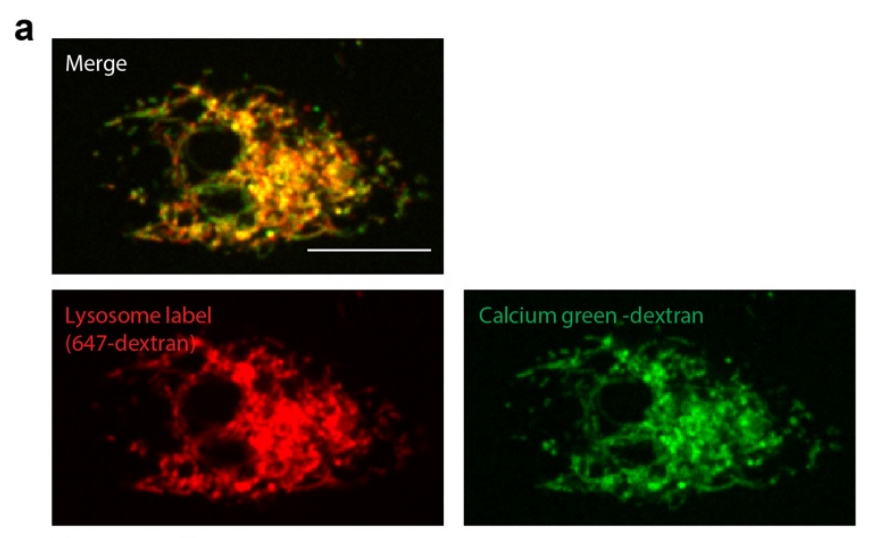

b
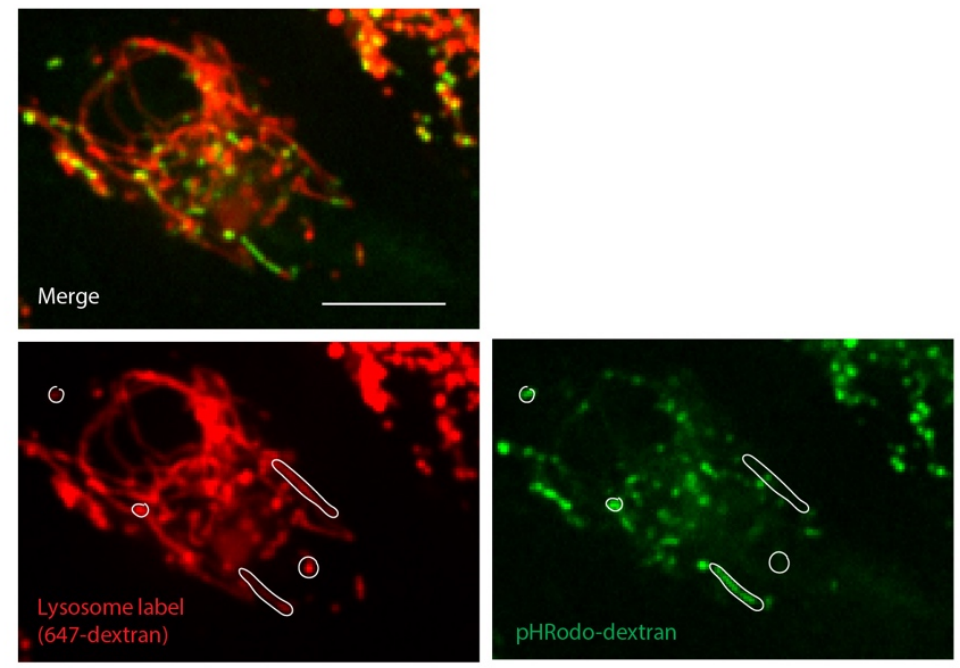

c

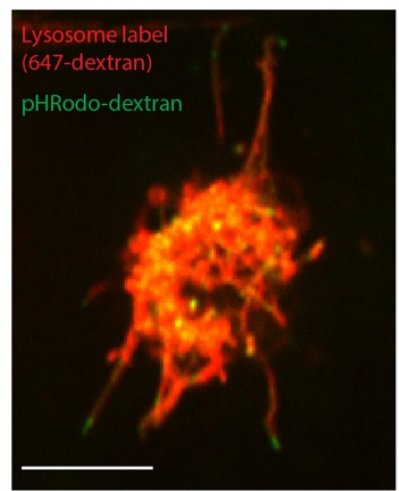

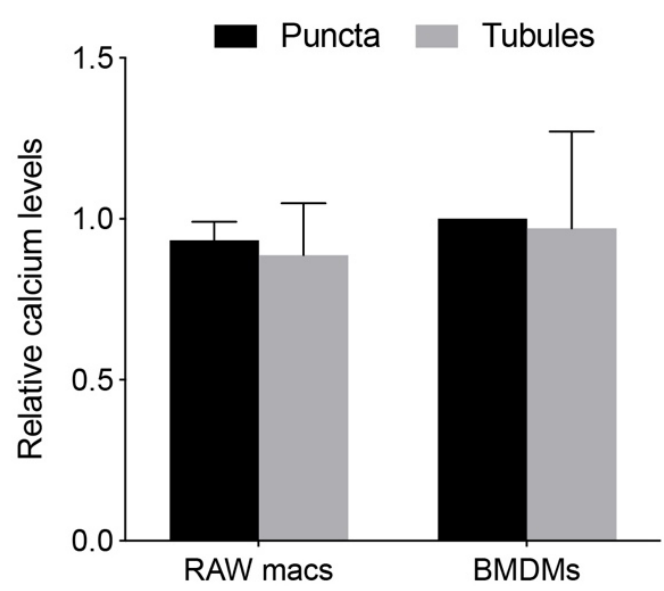

Cell Type

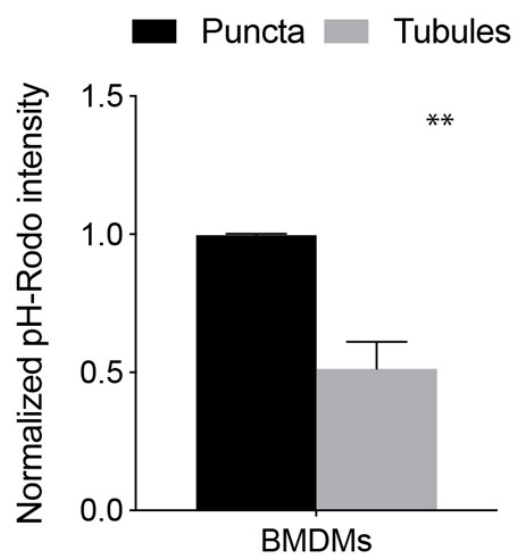


Figure 6.4: Luminal properties in tubular and punctate lysosomes differ in activated phagocytes.

(a) (left) BMDM lysosomes were co-labeled with Alex647-dextran (loading dextran) and calcium green-1 dextran using the standard pulse-chase lysosome labeling technique. Following lysosomal labeling, cells were activated with LPS for 2 hours to induce tubulation. (right) Quantification of relative calcium levels in tubules and punctate lysosomes in RAW and primary macrophages. Calcium green-1 fluorescence was normalized to the loading dextran for each ROI measured. There is no apparent difference between calcium levels in tubular and punctate lysosomes. (b) (right) BMDM lysosomes were co-labeled with Alex647-dextran (loading dextran) and $\mathrm{pH}$ sensitive pHRodo dextran using standard pulse-chase lysosome labeling technique. Cells were then activated with LPS for 2 hours to induce tubulation. ROIs in white high-light sample displaying differential pHRodo labeling in tubules and puncta. (left) pHRodo quantification of tubular and punctate lysosomes in BMDMs and BMDCs, where pHRodo intensity was normalized to the loading dextran. pHRodo intensity appears to decrease in BMDM lysosome tubules compared to puncta, suggesting a difference in lysosomal $\mathrm{pH}$. (c) Micrograph of cells labeled as in $\mathbf{b}$. Yellow ROIs indicate regions where strongly-labeled pHRodo puncta localize to the tips of tubular lysosomes. At least 10 tubules and puncta were measured across 20 cells for each experiment $(\mathrm{n}=3)$. Data are represented as averaged pHRodo intensity or calcium levels normalized to punctate lysosomes. ANOVA analysis was performed, where * indicates a statistically significant difference $(\mathrm{p}<0.05)$. Scale bar $=10 \mu \mathrm{m}$. 
Figure 6.5

a

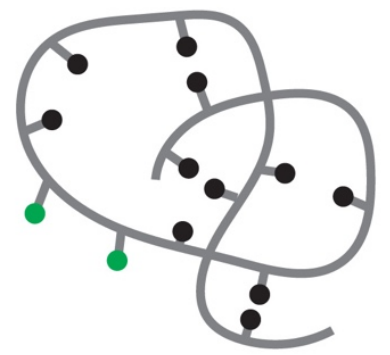

DQ-BSA

(BODIPY fluorescence quenching)
Fluorescent products

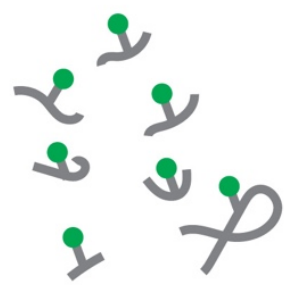

Hydrolytic enzymes

Cleaved peptide (Relieved queching)

b
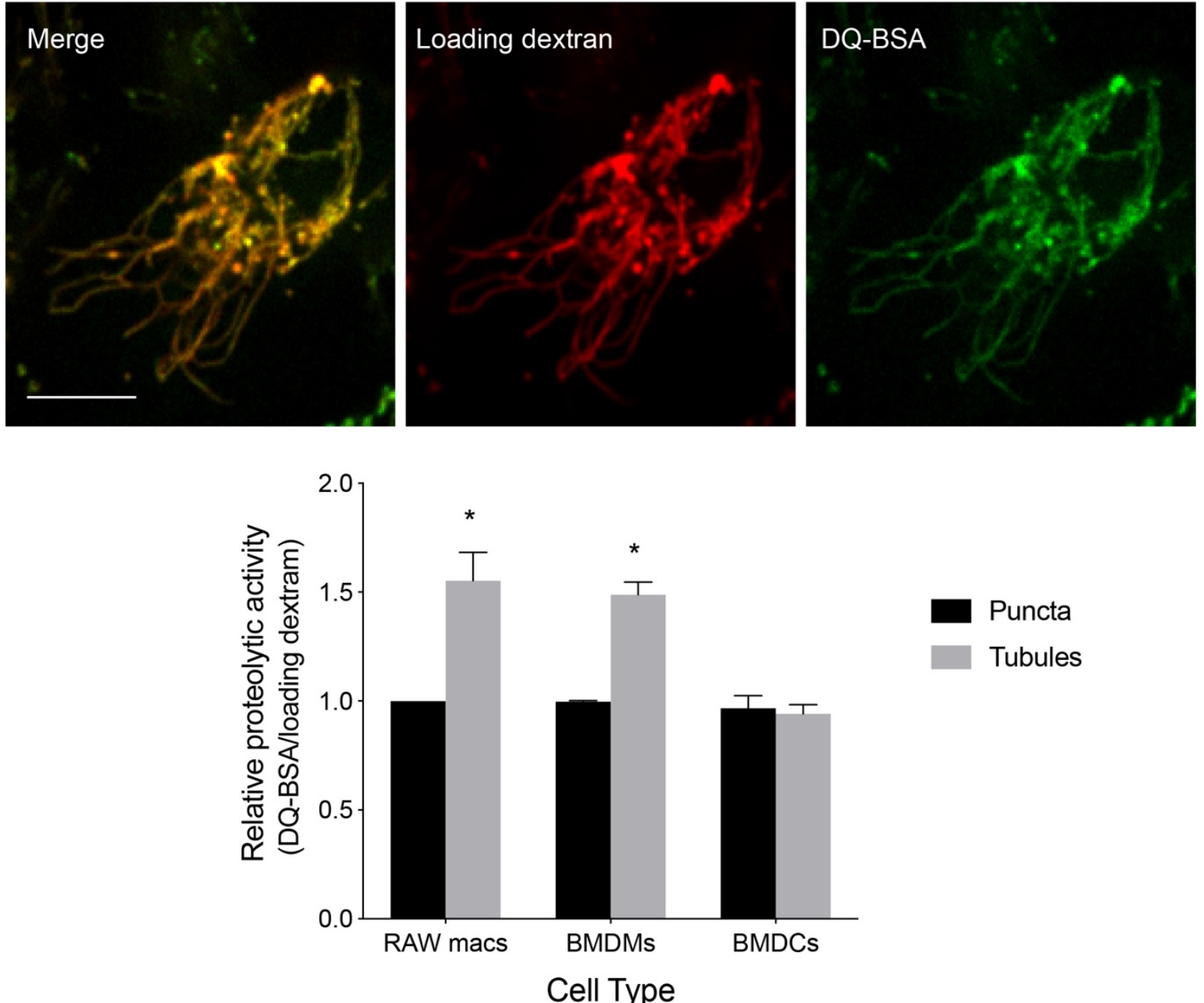

Puncta

Tubules

Cell Type 
Figure 6.5: Tubular lysosomes may be more proteolytic than punctate lysosomes in macrophages.

(a) Schematic of DQ-BSA proteolytic assay. (b) Lysosomes in BMDMs were co-labeled with DQBSA and Alexa-546 loading dextran using a standard pulse-chase lysosome labeling technique. Following lysosomal labeling, cells were activated with LPS for 2 hours to induce tubulation. Graph showing relative proteolytic activity of tubular and puncta lysosome. DQ-BSA fluorescence was normalized to the loading dextran for at least 10 tubules and puncta across 20 cells, for each experiment $(n=3)$. Data are represented as average proteolytic activity normalized to punctate lysosomes. ANOVA analysis was performed, where * indicates a statistically significant difference $(\mathrm{p}<0.05)$. Scale bar $=10 \mu \mathrm{m}$. 
Chapter 7: Conclusion 
In this dissertation I present two major projects investigating the mechanisms that control organelle identity and adaptation in phagocytic immune cells.

First, I present a novel mechanism showing that PtdIsn(3)P signal termination is controlled by the acidification of phagosomes and endosomes. We showed that organelles employ a $\mathrm{H}^{+}$ gradient across their membranes triggering the dissociation of the Vps34 complex II and depletion of PtdIns(3)P, coupling the progressive acidification of phagosomes and endosomes to the disappearance of PtdIns(3)P from these organelles as they mature. We also show that target length can also impact maturation. As proton leakage into the extracellular space permits $\operatorname{Ptd} \operatorname{Ins}(3) \mathrm{P}$ to persist on the tPC membrane, despite the loss of other early endosomal markers and acquisition of endolysosomal properties. When tPCs become sufficiently long or seal into a phagosome, then a $\mathrm{H}^{+}$gradient is generated that signals PtdIns(3)P depletion. Ultimately, this mechanism may be of relevance for the many cellular processes governed by PtdIns(3)P and its effector proteins. Indeed PtdIns(3)P-binding proteins not only play roles in vesicular trafficking and protein sorting along the endosomal and phagosomal pathways, but also in cytokinesis and metabolic signalling. Our findings expand on the current knowledge of the machinery and events that control phagosome maturation and may have implications in better understanding how bacteria can exploit these mechanisms to promote their pathogenesis and survival.

Secondly, I present a previously unknown mechanism of rapid lysosome expansion in activated phagocytes, whereby mTORC1 remodels lysosomes by modulating the translation of lysosomal mRNAs. Activated phagocytes dramatically reorganize their lysosomal system by expanding and forming a tubular lysosome network augmenting lysosomal holding capacity and their ability to internalize and retain more extracellular cargo, contributing to enhanced antigen presentation. We show that this process is governed by the mTOR-S6K-4E-BP axes, enhancing 
selective translation of lysosomal mRNAs. Collectively, we propose that mTORC1 and the regulated translation machinery is an important mechanism employed by cells to scale and adapt the size and volume of organelles in response to stress signals. We also present data suggesting that lysosome remodelling may have implications in adjuvant-induced antigen presentation. Together our data suggest that this pathway may play an important role in vaccine development, as well as, further our understanding on the cell biology of immune cells and how they can quickly adapt and scale organelles in response to environmental cues and cellular state. 
Appendix 
a

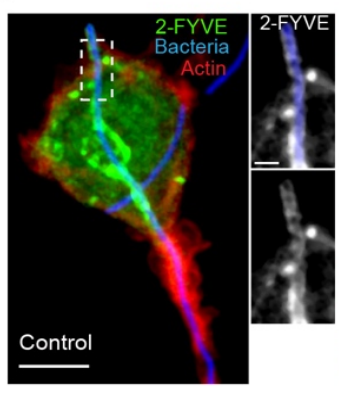

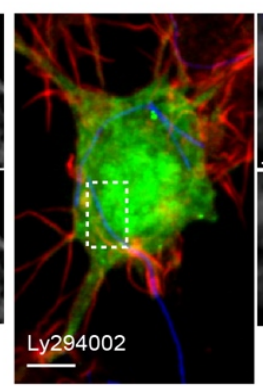
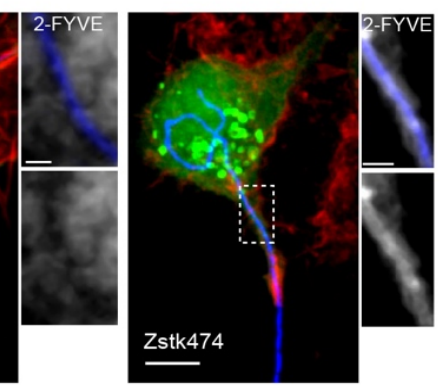

d

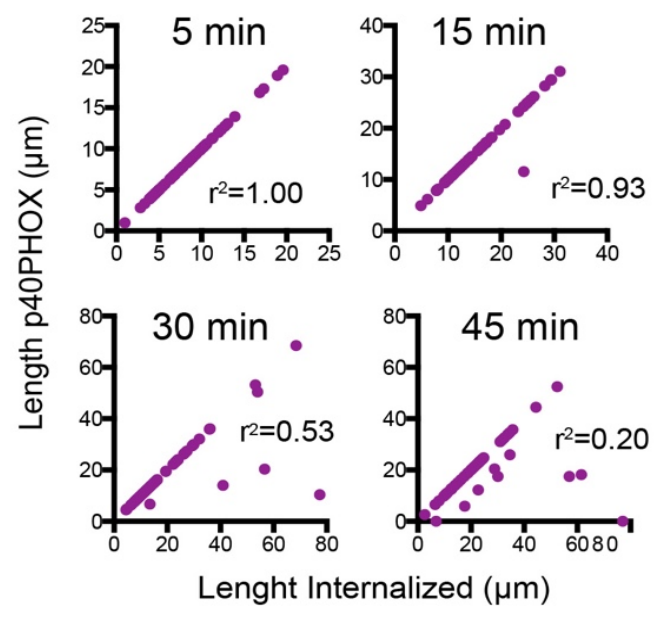

b

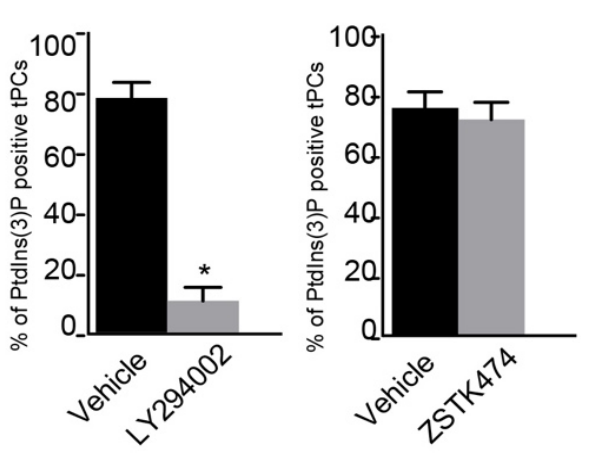

C

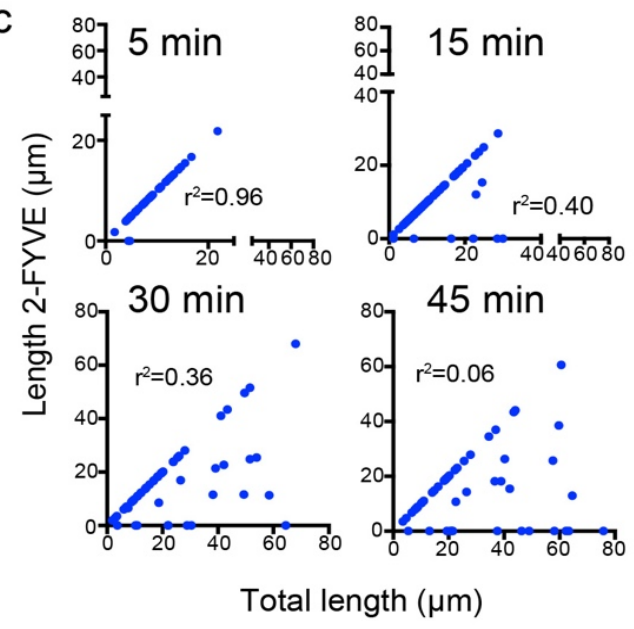

Figure A1: PI3K and tPC length-dependent recruitment of 2FYVE-GFP and p40PX-GFP.

(a) RAW cells expressing 2FYVE-GFP (green) were treated with DMSO (control), $100 \mu \mathrm{M}$ LY294002, or $1 \mu \mathrm{M}$ ZSTK4741 h prior to the onset of phagocytosis. (b) 2FYVE-GFP recruitment to tPCs were scored in all treatments. Data shown are means \pm SEMs from three independent experiments ( $\mathrm{n}=35$ for each). $\mathrm{P}<0.05$. Bars $=5 \mu \mathrm{m}$, and $1 \mu \mathrm{m}$ for the enlarged areas. (c, d) RAW macrophages expressing 2-FYVE and p40PX-GFP were challenged with f-LP and fixed after 5, 15,30 and $45 \mathrm{~min}$ of phagocytosis. The length of filamentous bacteria positive for each probe was plotted against the bacterial length internalized at each time point. The corresponding correlation coefficients $\left(\mathrm{r}^{2}\right)$ were calculated. This was measured in 3 independent experiments, $n=30$ for each condition. (Note: Figure experiment and data completed by Amriya Naufer). 
a
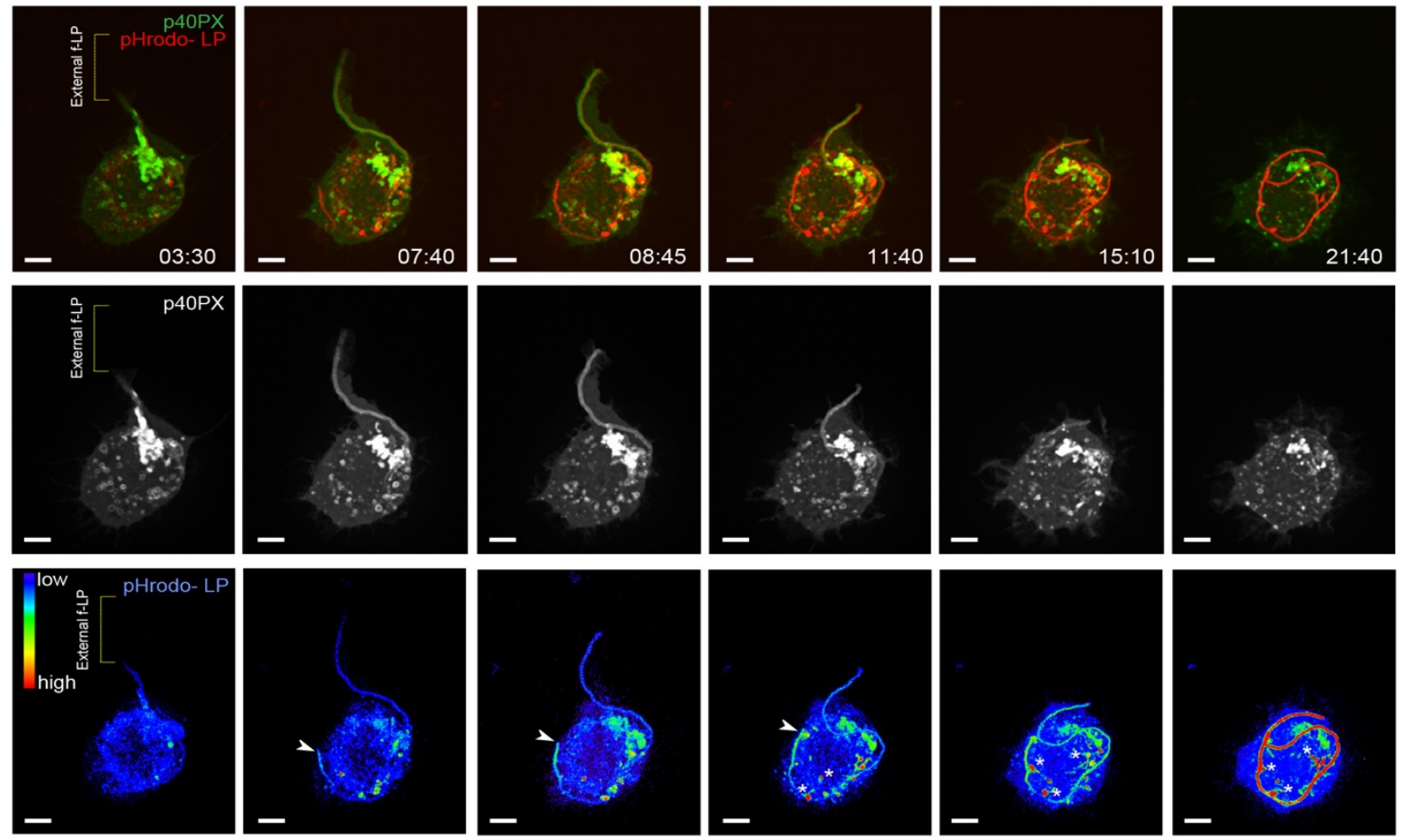

pHrodo

p40PX-GFP

b
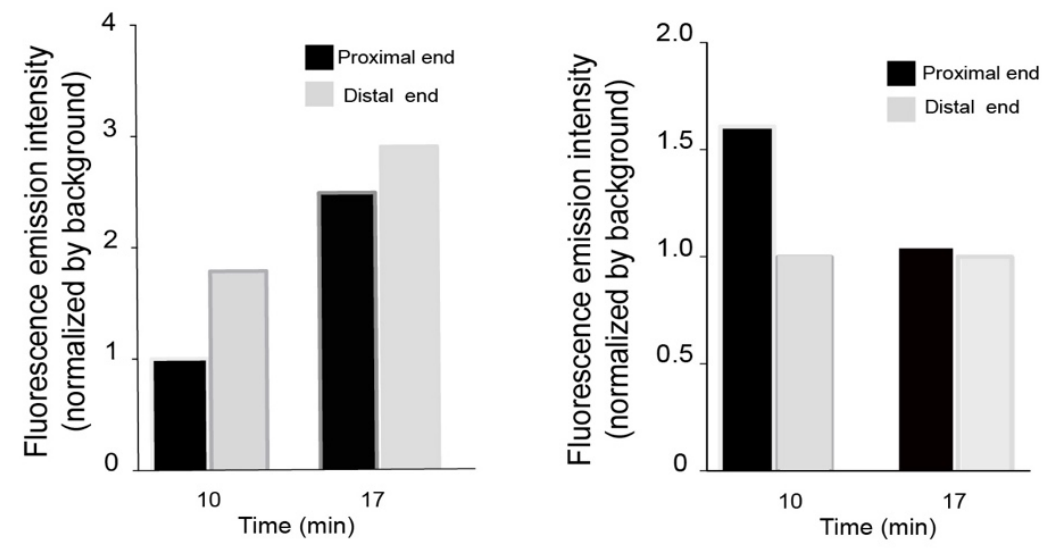

Figure A2: Loss of PtdIns(3)P is correlated with the acidification of distal tPCs.

(a) Evolution of p40PX-GFP and pHrodo fluorescence in growing tPCs. RAW macrophages expressing p40PX-GFP (green in top white in middle) challenged with pHrodo-LP and imaged live. pHrodo fluorescence (red: top row \& pseudo: bottom row, where blue is neutral pH and red is acidic $\mathrm{pH}$ ). Arrowheads in lower panel indicates distal tip of the tPCs. Asterisk indicates bacterial-associated pHrodo fluorescence extracted into cytoplasmic vesicles as the tPC remodels during internalization. Scale bars $=5 \mu \mathrm{m}$. (b) Relative pHRodo and p40PX-GFP fluorescence at distal and proximal tPCs, at 10 and 17 min of phagocytosis. (Note: Experiment by A. Naufer). 
a
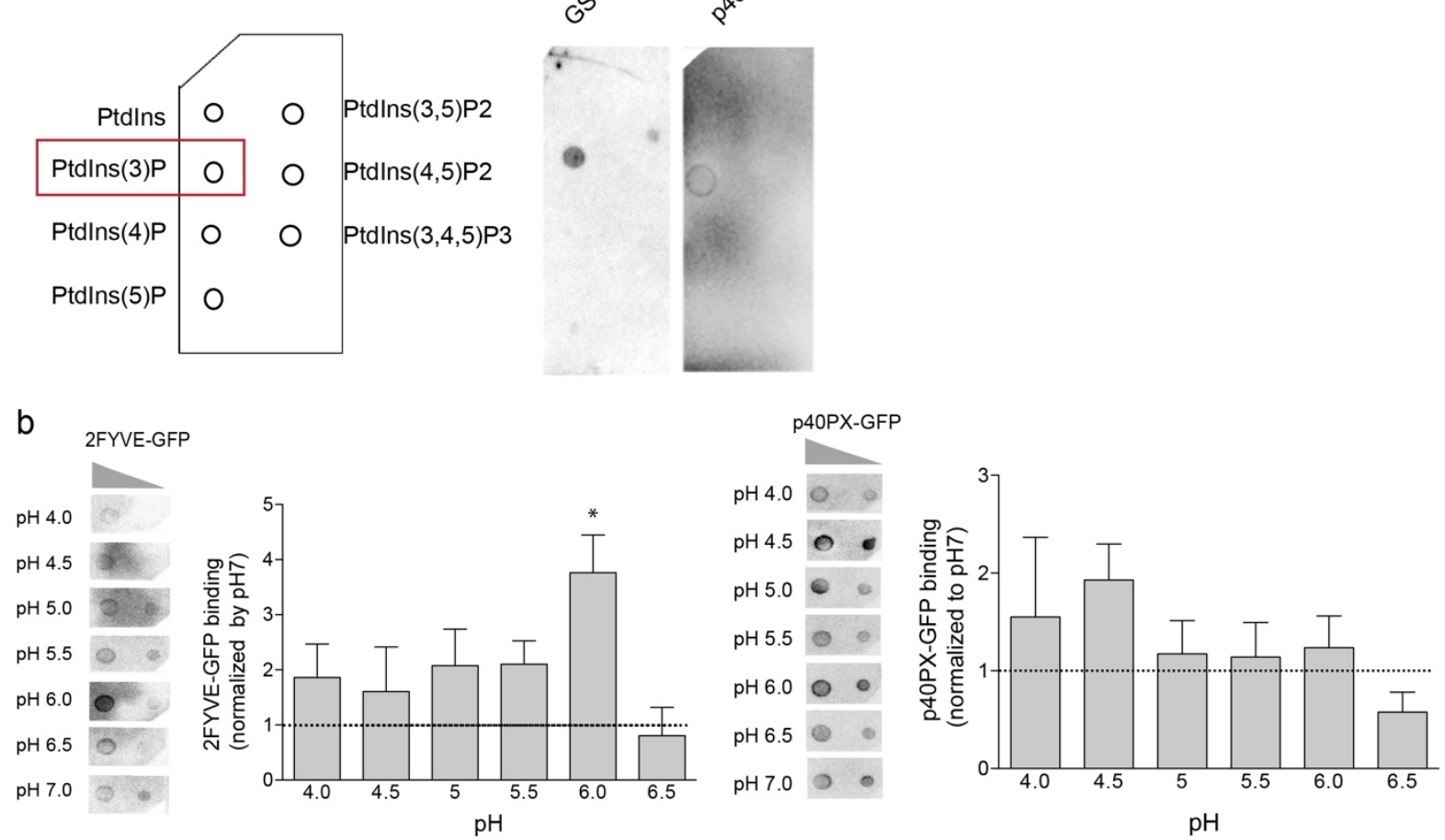

Figure A3: 2FYVE-GFP and p40PX-GFP respond to $\mathrm{pH}$.

(a) Recombinant GST-GFP-2FYVE, PX-GFP-V5-His6 and GST-GFP fusion protein purification was done as described in (Naufer et al., 2018). The indicated phosphoinositides (200 pmoles) were spotted onto nitrocellulose membranes and protein lipid overlays were performed using $0.5 \mu \mathrm{g} / \mathrm{ml}$ GST-GFP-2FYVE or PX-GFP-V5-His6 recombinant protein preparations. (b) Protein-lipid overlay (PLO) using recombinant GST-GFP-2FYVE and PX-GFP-V5-His6, and membranes containing 400 and 200 pmols of PtdIns(3)P. Representative experiment of three independent experiments performed with different preparations of recombinant protein. Right, densitometry of spots from each PLO expressed relative to that observed at $\mathrm{pH}: 7$ from three independent experiments. Results expressed as average $\pm \operatorname{SEM}(* \mathrm{p}<0.01)$. (Note: Experiment and data by Suriakarthiga Ganesan \& Vanina Zaremberg). 
Figure A4

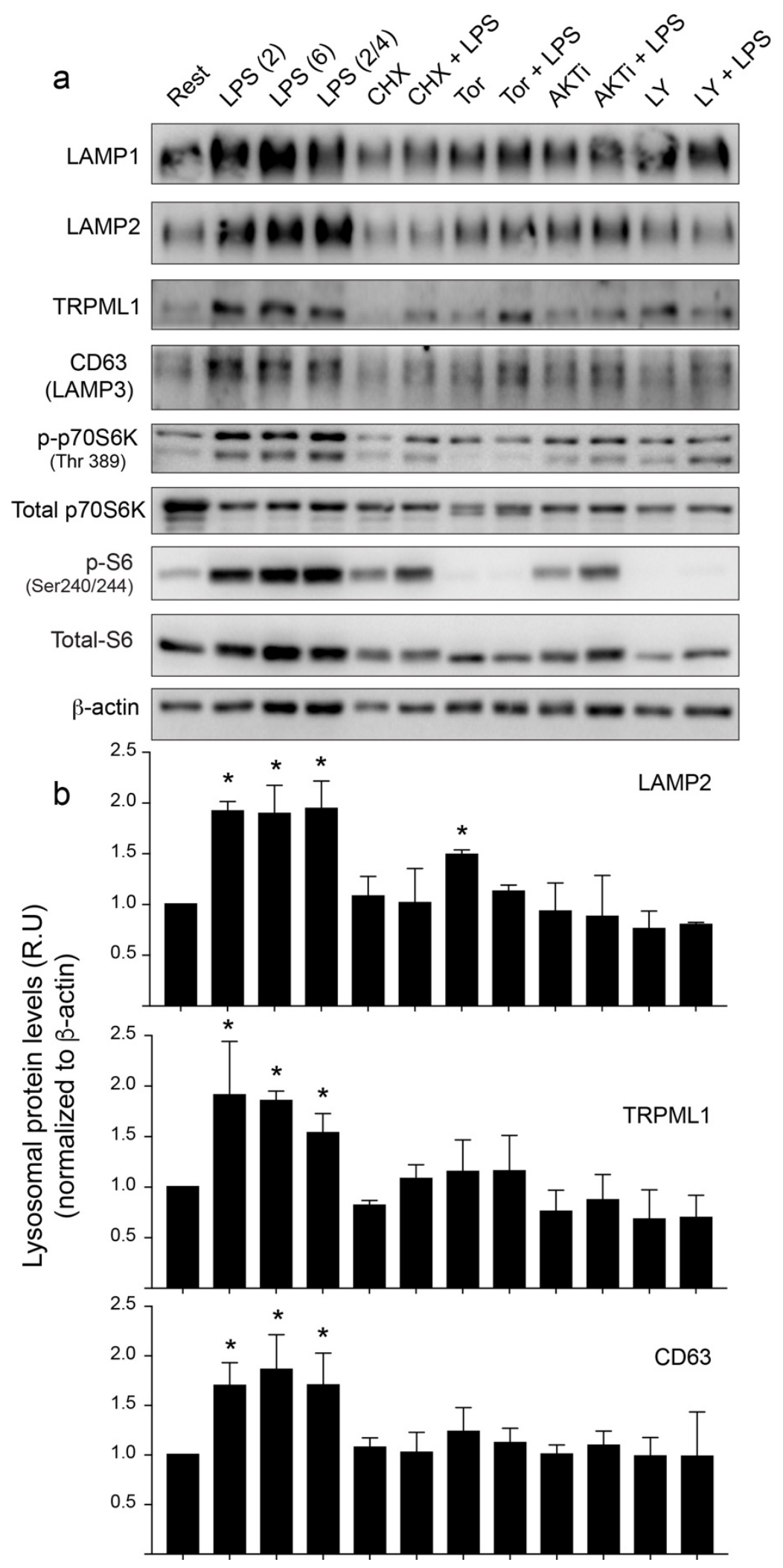


Figure A4: LPS increases lysosomal protein synthesis through mTOR and S6K.

(a) Western blot analysis of additional lysosomal proteins from whole cell lysates of resting primary macrophages or macrophages exposed to the indicated combinations and time of LPS, cycloheximide (CHX), Torin1, LY258470 (LY), AKT inhibitor (AKTi). (b) Quantification of Western blots showing the levels of LAMP2, TRPML1 and CD63 normalized to actin. Data shown as the mean \pm SEM from at least 3 independent experiments. For A and B, " $2 / 4$ " indicates cells stimulated with $2 \mathrm{~h}$ of LPS, followed by a $4 \mathrm{~h}$ chase, whereas 2 and $6 \mathrm{~h}$ represent cells continuously exposed to LPS. 
Figure A5
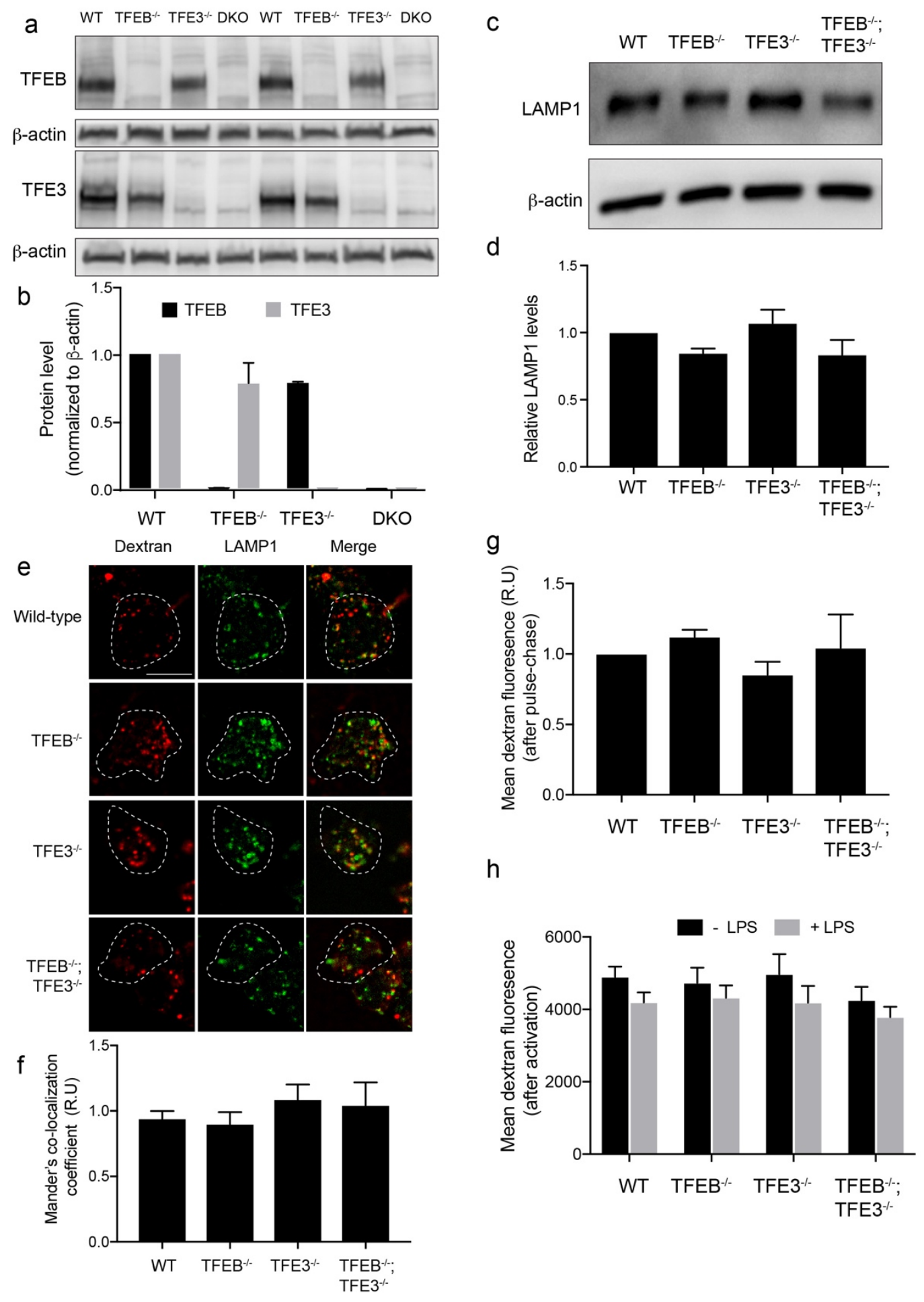
Figure A5: Basal lysosome properties and trafficking is indistinguishable in wild-type RAWs and strains deleted for TFEB and/or TFE3.

(a-b) Western blot analysis of TFEB, TFE3 and double deleted cell-lines. (c) LAMP1 levels in whole cell lysates from wild-type and deletion mutants of TFEB and/or TFE3. (d) Quantification of LAMP1 levels in knock-out cells. LAMP1 levels were normalized to $\beta$-actin to control for loading. Statistical analysis using ANOVA analysis, determined that LAMP1 levels did not vary across strains. (e) Co-localization of dextran and LAMP1 in wild-type and deletion strains. Right, middle and left panels show dextran (red), endogenous LAMP1 (green) and merge, respectively. Scale bar $=5 \mu \mathrm{m}$. (f) Mander's coefficient of dextran co-localizing in LAMP1 structures. Data shown as relative units (R.U), normalized to wild-type strain. (g) Pinocytosis label after a 1 h pulse and $1 \mathrm{~h}$ chase of fluorescent dextran in resting wild-type and deletion RAW strains, measured by microscopy and image analysis. Mean fluorescence intensity was normalized to wild-type strain and is represented as relative units (R.U). (h) Dextran fluorescence in RAW and deletion strains 2 $\mathrm{h}$ after LPS exposure or vehicle. For all data, shown are the mean \pm SD from at least three independent experiments.(Experiment and data for $\mathbf{c}, \mathbf{d}, \mathbf{g}, \mathbf{h}$ was completed by Jacqueline Diaz) 
Figure A6

a
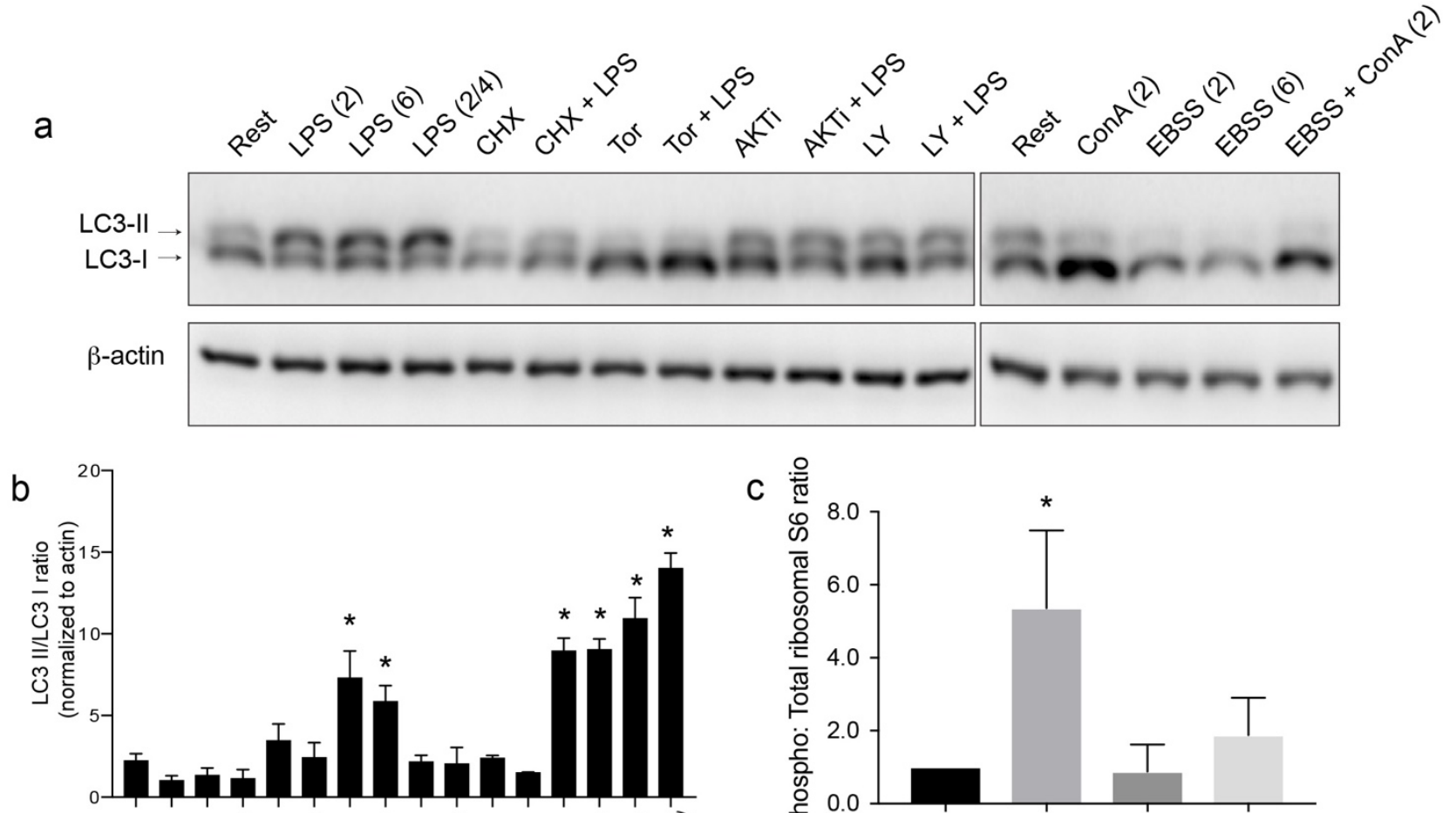

C 오중
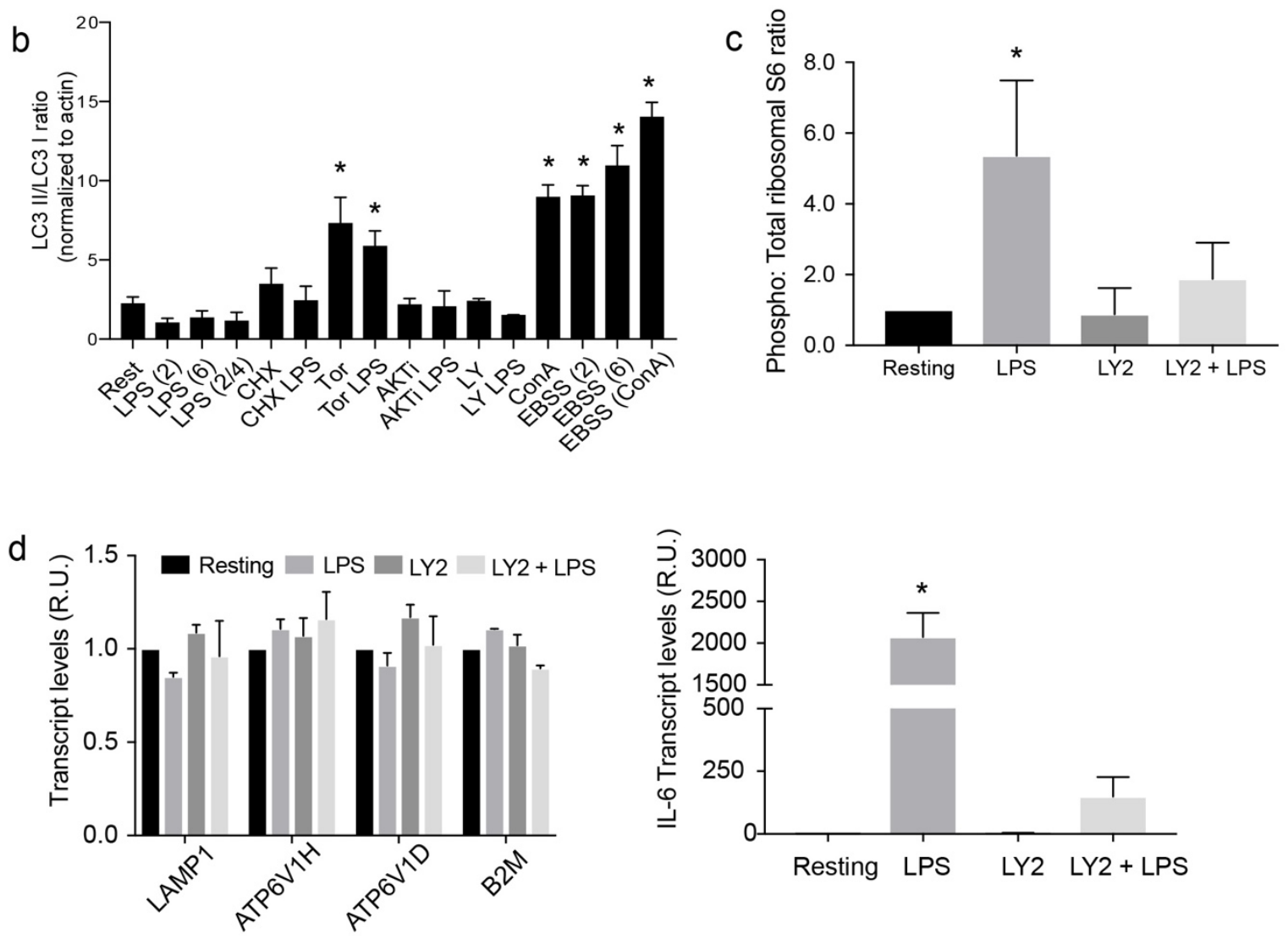
Figure A6: Impact of pharmacological inhibitors on autophagy induction and LY258470 treatment on S6K activity during and general protein synthesis.

(a) Western blot analysis of LC3I to LC3II conversion to measure treatment effect on autophagy induction. BMDMs were activated with LPS in the presence or absence of mTOR and S6K inhibitors for the time points indicated. Concanamycin A (ConA) and EBSS treatment was used as a positive control for autophagy induction. (b) Quantification of a from three independent experiments. Ratio of LC3II to LC3I levels was normalized to actin loading control. (c) Normalized ratio of phosphorylated ribosomal S6 to total ribosomal S6 as depicted in Fig. 8d, in primary macrophages treated with LY258470 (LY2) alone, or co-incubated with LPS for $2 \mathrm{~h}$. Shown is the mean \pm standard deviation of the mean from five independent blots. (d) Relative mRNA levels of select lysosomal genes (right) or interleukin-6 (left) in LPS and/or LY2 treated primary macrophages relative to Abt1 housekeeping gene and normalized against resting cells. Quantification was done with qRT-PCR by measuring the $\Delta \Delta \mathrm{Ct}$ as described in methods. Shown is the mean \pm SEM from four independent experiments. 
$\rightarrow$ Vehicle $\rightarrow$ Torin1 $(2 \mathrm{~h})$
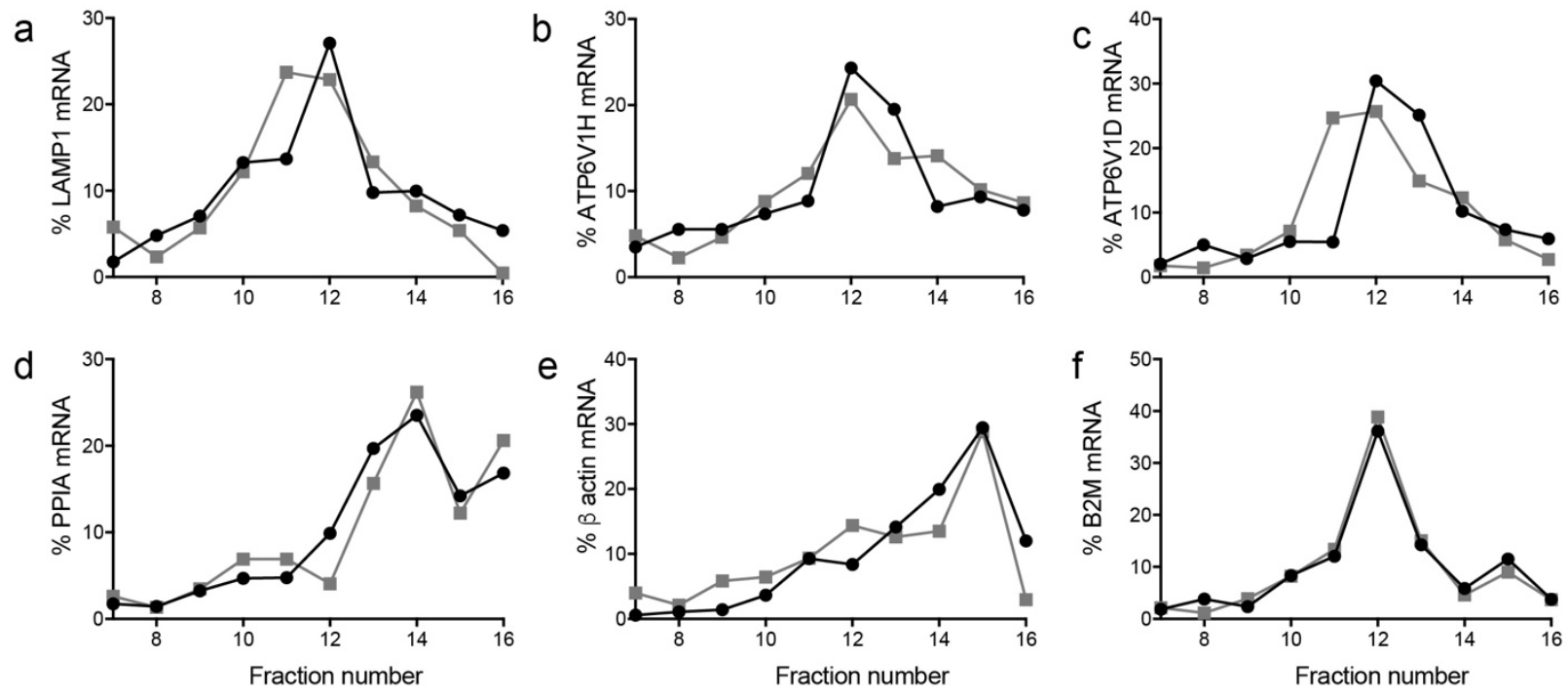

Figure A7: The effects of Torin1 on RAW macrophage protein synthesis.

a-d. Percent of target mRNA (a: LAMP1, b: ATP6V1H, c: ATP6V1D d: PPIA, e: $\beta$-actin and f. $\mathrm{B} 2 \mathrm{M})$ associated with each ribosome fraction in resting and torin $1(2 \mathrm{~h} ; 100 \mathrm{nM})$ treated cells. From data set presented in Figure 4.9. 
Figure A8.
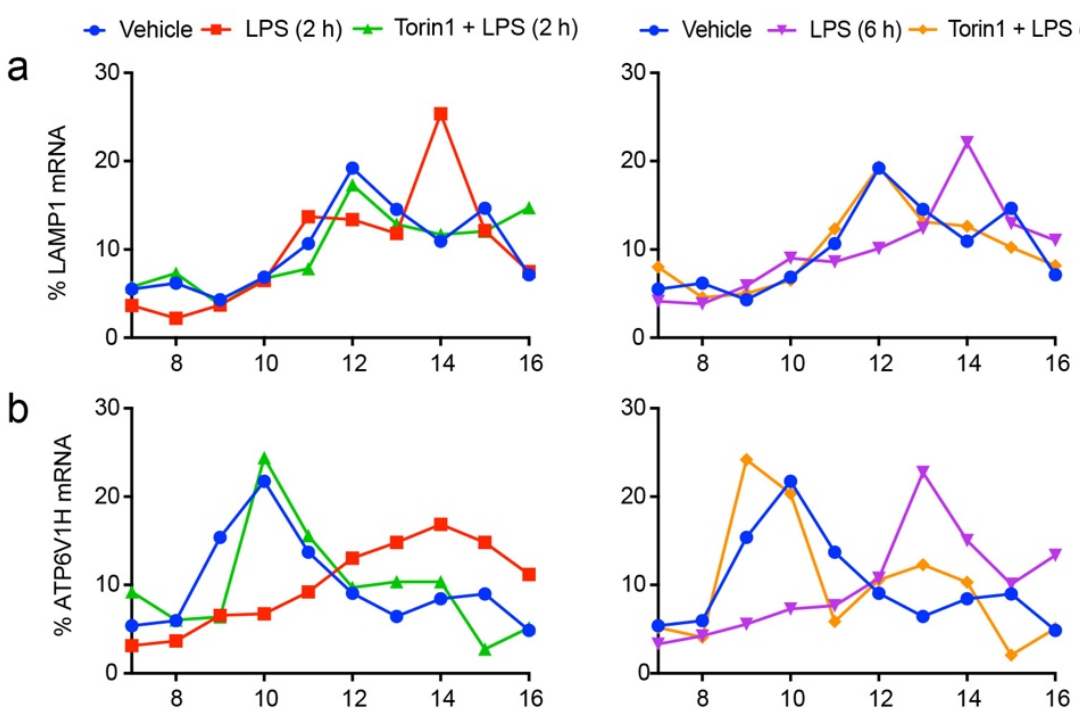

C
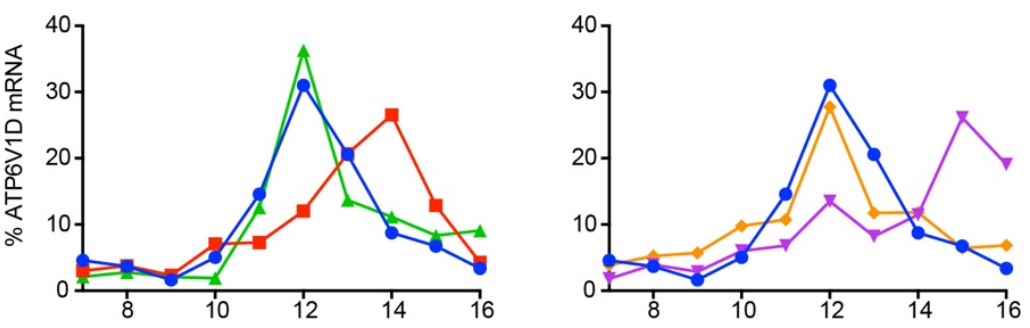

d

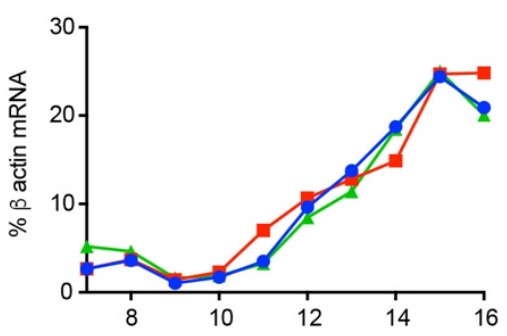

e
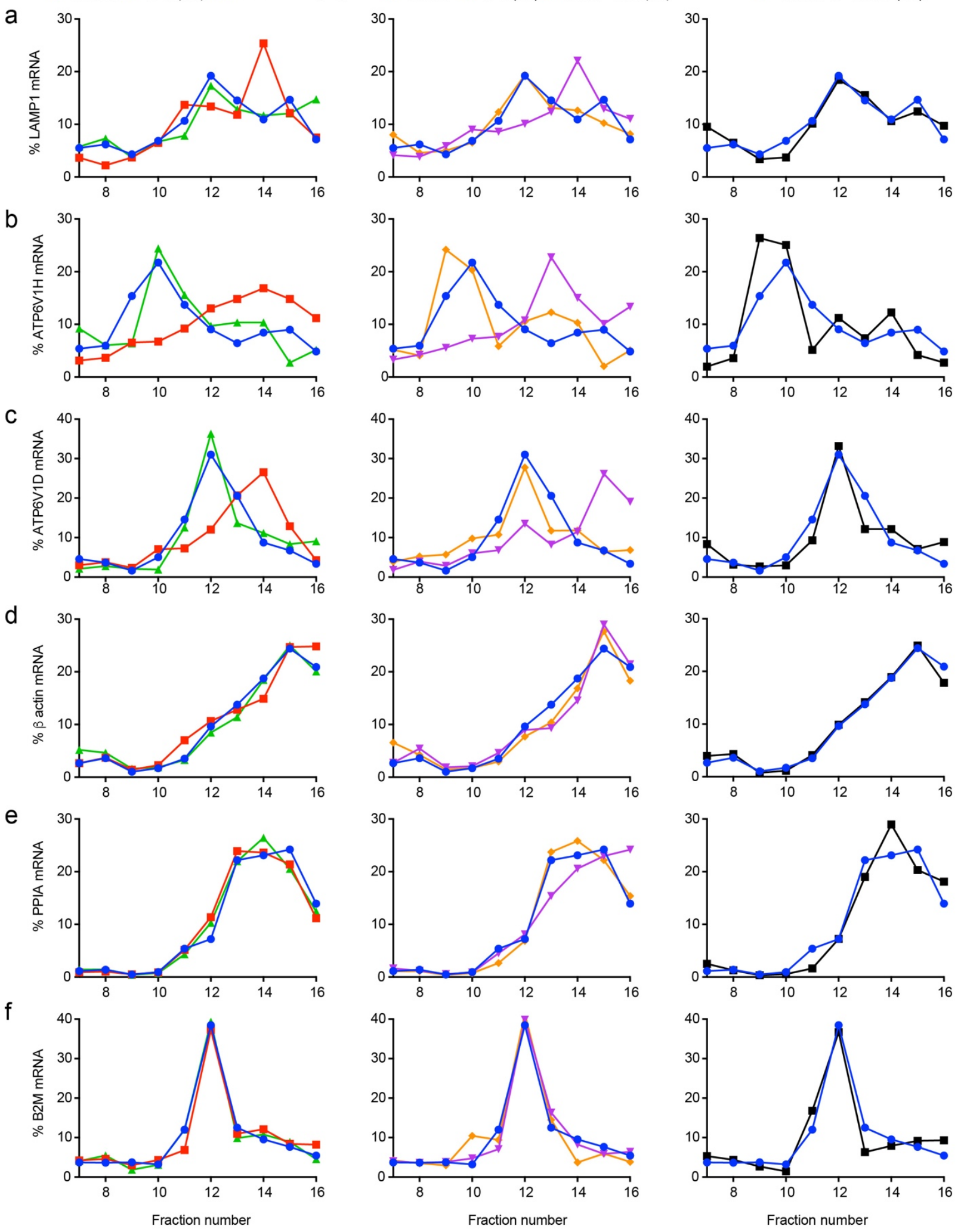
Figure A8: Polysome profiling of RAW macrophages: additional replicate data.

Percent of target mRNA (a: LAMP1, b: ATP6V1H, c: ATP6V1D d: $\beta$-actin e: PPIA and f. B2M) associated with each ribosome fraction in resting, LPS-treated macrophages and macrophages coexposed to LPS and torin1, or treated with torin1 alone. Left, middle and right panels show 2 h, 6 $\mathrm{h}$ and torin $1(2 \mathrm{~h})$ treatments, respectively. Shown, is an additional biological replicate of the experiment described in Figure 4.9. 

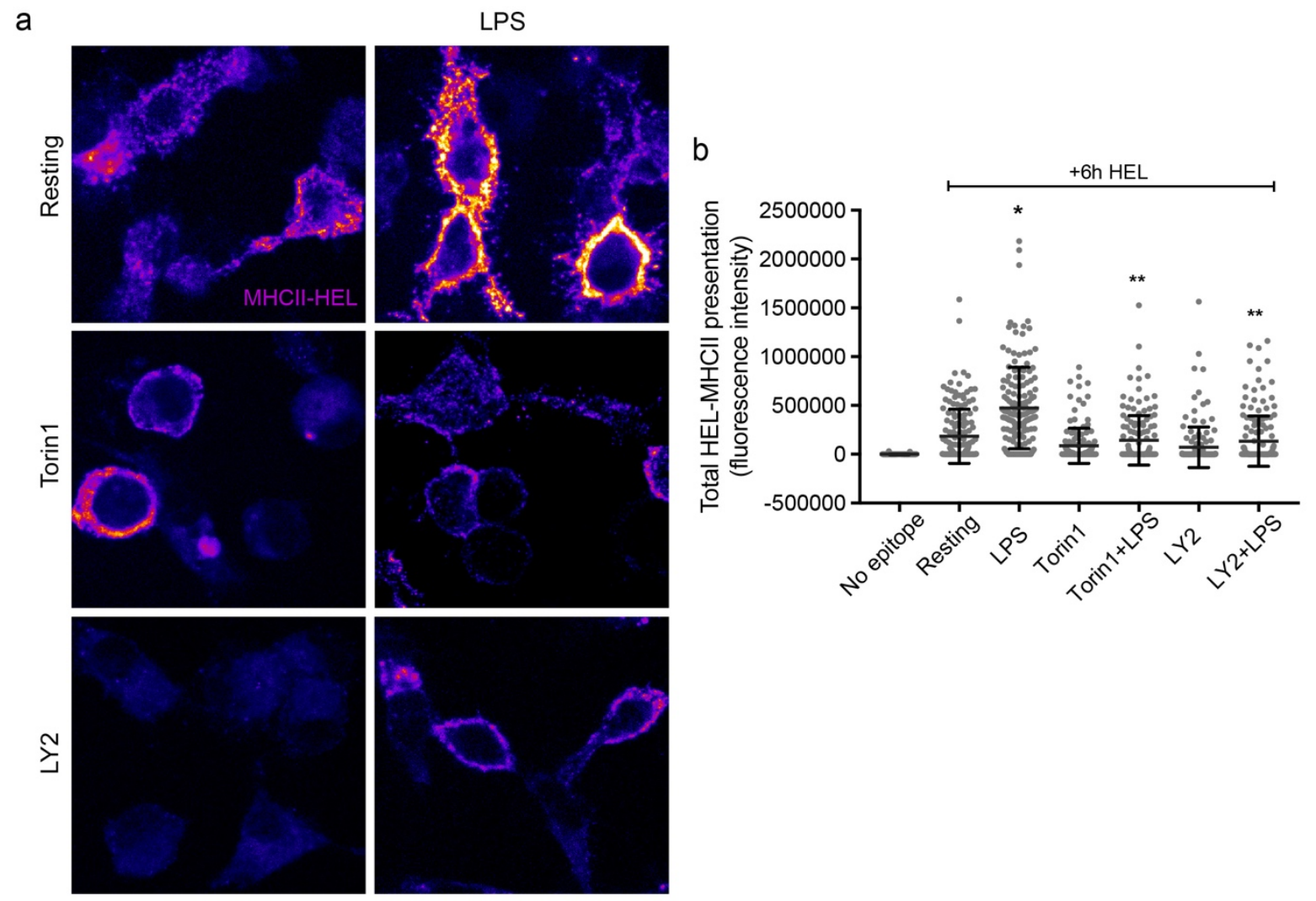

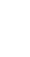

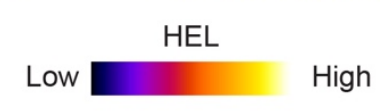

Figure A9: Effect of LY258470 and Torin1 treatments on HEL presentation by BMDCs.

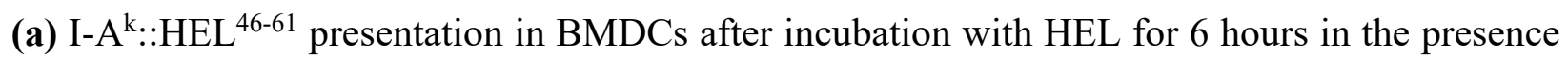
and/or absence of LPS, torin1 and LY2. I-A $:: \mathrm{HEL}^{46-61}$ cell surface levels were detected by staining unpermeabilized cells with the monoclonal antibody Aw3.18.14 (b) Quantification of total average

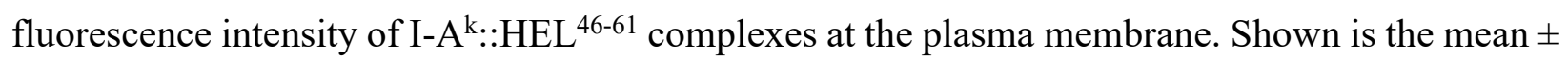
SD from three experiments, where 50-100 cells were quantified for each. Data was analyzed using ANOVA, whereby * indicates a difference compared to the Resting + HEL condition and ** indicates a difference compared to HEL + LPS $(p<0.05)$. Scale bar $=15 \mu \mathrm{m}$. Colour scale: $0-12000$ (low-high). 
Figure A10
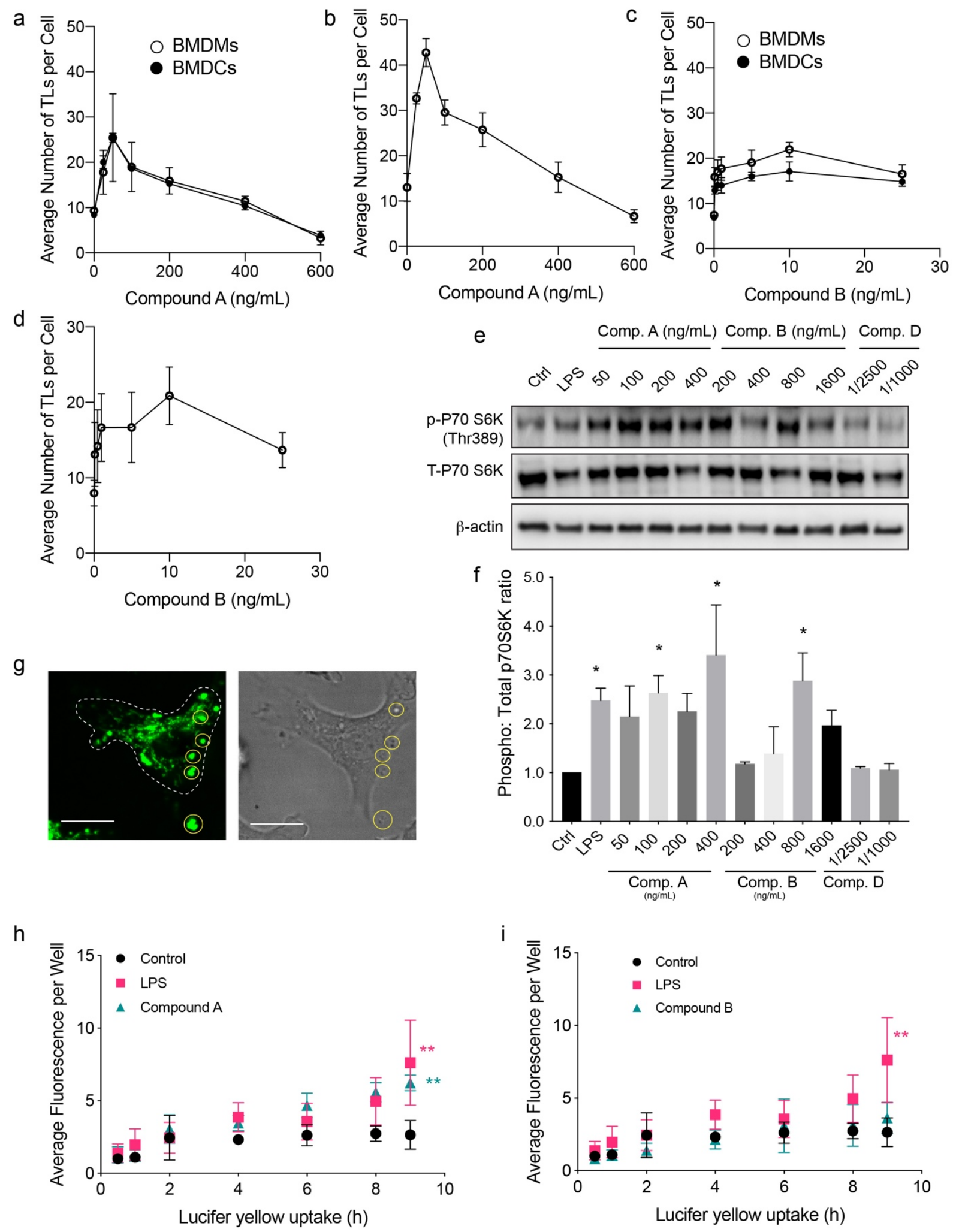
Figure A10: Adjuvant-induced lysosome remodelling supporting data.

(a-d) Lysosomes in primary BMDMs and BMDCs were labeled using standard pulse-chase assay and then stimulated with varying doses of compound A or B. The average number of lysosome tubules per cell longer than $4 \mu \mathrm{m}$ in length were determined in BMDMs and BMDCs differentiated from (a, b) $\mathrm{C} 57 \mathrm{BL} / 6 \mathrm{~J}$ and $(\mathbf{c}, \mathbf{d}) \mathrm{C} 3 \mathrm{H} / \mathrm{HeN}$ mice bone-marrow. Shown is the mean $\pm \mathrm{SD}$ from three experiments, where 50-100 cells were quantified for each. (Experiment and data analysis completed by Neha Chauhan).

(e-f) Western blot from BMDC whole-cell lysis showing mTOR activation following compound A and B stimulation. (e) Levels of phosphorylated p70-S6K, total p70-S6K and $\beta$-actin following compound A and B treatment. (f) Quantification showing the ratio of phosphorylated p70-S6K to total $\mathrm{p} 70$-S6K, normalized to $\beta$-actin. Shown is the mean \pm SD from three experiments. Data was analyzed using ANOVA, whereby * indicates a difference compared to the Resting $(\mathrm{p}<0.05)$.

(g) Fluorescently labeled lysosomes (left) and DIC-phase contrast (right) of BMDC stimulated with compound D. Auto-fluorescent compound D molecules outlined in yellow circles.

(h-i) Fluid-phase uptake of lucifer yellow overtime in BMDCs treated with compound A and LPS (h) or compound B and LPS (i). Shown is the mean fluorescence of lucifer yellow per well $\pm \mathrm{SD}$ from three experiments. Experiment performed used standard fluorescent plate reader. Data was analyzed using ANOVA, whereby ** indicates a difference compared to the Resting $(\mathrm{p}<0.05)$. (Experiment and data analysis completed by Neha Chauhan). 
References

A-Gonzalez, N., Bensinger, S. J., Hong, C., Beceiro, S., Bradley, M. N., Zelcer, N., ... Castrillo, A. (2009). Apoptotic cells promote their own clearance and immune tolerance through activation of the nuclear receptor LXR. Immunity, 31(2), 245-258. https://doi.org/10.1016/j.immuni.2009.06.018

Abe, N., Inoue, T., Galvez, T., Klein, L., \& Meyer, T. (2008). Dissecting the role of PtdIns(4,5)P2 in endocytosis and recycling of the transferrin receptor. Journal of Cell Science, 121(9), 1488-1494. https://doi.org/10.1242/jcs.020792

Abrahamson, D. R., \& Rodewald, R. (1981). Evidence for the sorting of endocytic vesicle contents during the receptor-mediated transport of IgG across the newborn rat intestine. The Journal of Cell Biology, 91(1), 270-280. https://doi.org/10.1083/jcb.91.1.270

Adachi, T., Arai, K., \& Ohkuma, S. (1996). A comparative study of (Ca(2+)-Mg2+)-ATPase on the lysosomal membrane and ecto-ATPase on the plasma membrane from rat liver. Biological \& Pharmaceutical Bulletin, 19(10), 1291-1297.

Amaral, E. P., Riteau, N., Moayeri, M., Maier, N., Mayer-Barber, K. D., Pereira, R. M., ... Andrade, B. B. (2018). Lysosomal Cathepsin Release Is Required for NLRP3-Inflammasome Activation by Mycobacterium tuberculosis in Infected Macrophages. Frontiers in Immunology, 9, 1427. https://doi.org/10.3389/fimmu.2018.01427

Andrei, C., Dazzi, C., Lotti, L., Torrisi, M. R., Chimini, G., \& Rubartelli, A. (2013). The Secretory Route of the Leaderless Protein Interleukin 1beta Involves Exocytosis of Endolysosomerelated Vesicles. Molecular Biology of the Cell. https://doi.org/10.1091/mbc.10.5.1463

Andrei, C., Margiocco, P., Poggi, A., Lotti, L. V., Torrisi, M. R., \& Rubartelli, A. (2004). From The Cover: Phospholipases C and A2 control lysosome-mediated IL-1 secretion: Implications for inflammatory processes. Proceedings of the National Academy of Sciences, 101(26), 9745-9750. https://doi.org/10.1073/pnas.0308558101

Andrews, N. W. (2002). Lysosomes and the plasma membrane: trypanosomes reveal a secret relationship. The Journal of Cell Biology, 158(3), 389-394. https://doi.org/10.1083/jcb.200205110 
Appelmans, F., Wattiaux, R., \& De Duve, C. (1955). Tissue fractionation studies. 5. The association of acid phosphatase with a special class of cytoplasmic granules in rat liver. The Biochemical Journal, 59(1951), 438-445.

Appelqvist, H., Waster, P., Kagedal, K., \& Ollinger, K. (2013). The lysosome: from waste bag to potential therapeutic target. Journal of Molecular Cell Biology, 5(4), 214-226. https://doi.org/10.1093/jmcb/mjt022

Askew, D., Gatewood, J., Olivas, E., Havenith, K., \& Walker, W. S. (1995). A subset of splenic macrophages process and present native antigen to naive antigen-specific CD4+ T-cells from mice transgenic for an alpha beta T-cell receptor. Cellular Immunology, 166(1), 62-70. https://doi.org/10.1006/cimm.1995.0008

Avni, D., Biberman, Y., \& Meyuhas, O. (1997). The 5' terminal oligopyrimidine tract confers translational control on TOP mRNAs in a cell type- and sequence context-dependent manner. Nucleic Acids Research, 25(5), 995-1001.

Backer, J. M. (2016). The intricate regulation and complex functions of the Class III phosphoinositide 3-kinase Vps34. Biochemical Journal, 473(15), 2251-2271. https://doi.org/10.1042/BCJ20160170

Bago, R., Malik, N., Munson, M. J., Prescott, A. R., Davies, P., Sommer, E., ... Alessi, D. R. (2014). Characterization of VPS34-IN1, a selective inhibitor of Vps34, reveals that the phosphatidylinositol 3-phosphate-binding SGK3 protein kinase is a downstream target of class III phosphoinositide 3-kinase. Biochemical Journal. https://doi.org/10.1042/BJ20140889

Balderhaar, H. J. k., \& Ungermann, C. (2013). CORVET and HOPS tethering complexes coordinators of endosome and lysosome fusion. Journal of Cell Science. https://doi.org/10.1242/jcs.107805

Balla, T. (2013). Phosphoinositides: tiny lipids with giant impact on cell regulation. Physiological Reviews, 93(3), 1019-1137. https://doi.org/10.1152/physrev.00028.2012

Banchereau, J., \& Steinman, R. M. (1998). Dendritic cells and the control of immunity. Nature, 392(March), 245-252. https://doi.org/10.1038/32588 
Bandyopadhyay, U., Kaushik, S., Varticovski, L., \& Cuervo, A. M. (2008). The chaperonemediated autophagy receptor organizes in dynamic protein complexes at the lysosomal membrane. Molecular and Cellular Biology, 28(18), 5747-5763. https://doi.org/10.1128/MCB.02070-07

Bar-Peled, L., Chantranupong, L., Cherniack, A. D., Chen, W. W., Ottina, K. A., Grabiner, B. C., ... Sabatini, D. M. (2013). A Tumor Suppressor Complex with GAP Activity for the Rag GTPases That Signal Amino Acid Sufficiency to mTORC1. Science, 340(6136), 1100-1106. https://doi.org/10.1126/science.1232044

Bar-Peled, Liron, \& Sabatini, D. M. (2014). Regulation of mTORC1 by amino acids. Trends in Cell Biology. https://doi.org/10.1016/j.tcb.2014.03.003

Bar-Peled, Liron, Schweitzer, L. D., Zoncu, R., \& Sabatini, D. M. (2012). Ragulator is a GEF for the rag GTPases that signal amino acid levels to mTORC1. Cell, 150(6), 1196-1208. https://doi.org/10.1016/j.cell.2012.07.032

Barois, N., De Saint-Vis, B., Lebecque, S., Geuze, H. J., \& Kleijmeer, M. J. (2002). MHC class II compartments in human dendritic cells undergo profound structural changes upon activation. Traffic. https://doi.org/10.1034/j.1600-0854.2002.31205.x

Bauerfeld, C. P., Rastogi, R., Pirockinaite, G., Lee, I., Huttemann, M., Monks, B., .. Samavati, L. (2012). TLR4-Mediated AKT Activation Is MyD88/TRIF Dependent and Critical for Induction of Oxidative Phosphorylation and Mitochondrial Transcription Factor A in Murine Macrophages. The Journal of Immunology, 188(6), 2847-2857. https://doi.org/10.4049/jimmunol.1102157

Beg, a a, Finco, T. S., Nantermet, P. V, \& Baldwin, a S. (1993). Tumor necrosis factor and interleukin-1 lead to phosphorylation and loss of I kappa B alpha: a mechanism for NF-kappa B activation. Molecular and Cellular Biology, 13(6), 3301-3310. https://doi.org/10.1128/MCB.13.6.3301.Updated

Behnia, R., \& Munro, S. (2005). Organelle identity and the signposts for membrane traffic. Nature, 438(7068), 597-604. https://doi.org/10.1038/nature04397

Bendayan, M., \& Gisiger, V. (2001). Demonstration of Acetylcholinesterase Molecular Forms in a Continuous Tubular Lysosomal System of Rat Pancreatic Acinar Cells. Journal of 
Histochemistry $\quad \& \quad$ Cytochemistry, $\quad 49(1), \quad 29-39$. https://doi.org/10.1177/002215540104900104

Bertho, N., Cerny, J., Kim, Y.-M., Fiebiger, E., Ploegh, H., \& Boes, M. (2003). Requirements for T Cell-Polarized Tubulation of Class II+ Compartments in Dendritic Cells. The Journal of Immunology, 171(11), 5689-5696. https://doi.org/10.4049/jimmunol.171.11.5689

Bilanges, B., Argonza-Barrett, R., Kolesnichenko, M., Skinner, C., Nair, M., Chen, M., \& Stokoe, D. (2007). Tuberous sclerosis complex proteins 1 and 2 control serum-dependent translation in a TOP-dependent and -independent manner. Molecular and Cellular Biology, 27(16), 5746-5764. https://doi.org/10.1128/MCB.02136-06

Blott, E. J., \& Griffiths, G. M. (2002). Secretory lysosomes. Nature Reviews Molecular Cell Biology, 3(2), 122-131. https://doi.org/10.1038/nrm732

Bluestone, J. A. (2011). Mechanisms of tolerance. Immunological Reviews, 241(1), 5-19. https://doi.org/10.1111/j.1600-065X.2011.01019.X

Blum, J. S., Wearsch, P. A., \& Cresswell, P. (2013). Pathways of Antigen Processing. Annual Review of Immunology, 31(1), 443-473. https://doi.org/10.1146/annurev-immunol-032712095910

Boes, M., Bertho, N., Cerny, J., Op den Brouw, M., Kirchhausen, T., \& Ploegh, H. (2003). T Cells Induce Extended Class II MHC Compartments in Dendritic Cells in a Toll-Like ReceptorDependent Manner. The Journal of Immunology, 171(8), 4081-4088. https://doi.org/10.4049/jimmunol.171.8.4081

Boes, Marianne, Cerny, J., Massol, R., Op den Brouw, M., Kirchhausen, T., Chen, J., \& Ploegh, H. L. (2002). T-cell engagement of dendritic cells rapidly rearranges MHC class II transport. Nature, 418(6901), 983-988. https://doi.org/10.1038/nature01004

Bone, L. N., Dayam, R. M., Lee, M., Kono, N., Fairn, G. D., Arai, H., .. Antonescu, C. N. (2017). The acyltransferase LYCAT controls specific phosphoinositides and related membrane traffic. Molecular Biology of the Cell, 28(1), 161-172. https://doi.org/10.1091/mbc.E16-090668

Botelho, R. J., \& Grinstein, S. (2011). Phagocytosis. Current Biology: CB, 21(14), R533-8. 
https://doi.org/10.1016/j.cub.2011.05.053

Bretou, M., Sáez, P. J., Sanséau, D., Maurin, M., Lankar, D., Chabaud, M., ... Lennon-Duménil, A.-M. (2017). Lysosome signaling controls the migration of dendritic cells. Science Immunology, 2(16), eaak9573. https://doi.org/10.1126/sciimmunol.aak9573

Brieger, K., Schiavone, S., Miller, F. J., \& Krause, K. H. (2012). Reactive oxygen species: From health to disease. Swiss Medical Weekly. https://doi.org/10.4414/smw.2012.13659

Brubaker, S. W., Bonham, K. S., Zanoni, I., \& Kagan, J. C. (2015). Innate Immune Pattern Recognition: A Cell Biological Perspective. Annual Review of Immunology (Vol. 33). https://doi.org/10.1146/annurev-immunol-032414-112240

Burd, C. G., \& Emr, S. D. (1998). Phosphatidylinositol(3)-phosphate signaling mediated by specific binding to RING FYVE domains. Molecular Cell, 2(1), 157-162.

Burman, C., \& Ktistakis, N. T. (2010). Regulation of autophagy by phosphatidylinositol 3phosphate. FEBS Letters. https://doi.org/10.1016/j.febslet.2010.01.011

Burnett, P. E., Barrow, R. K., Cohen, N. A., Snyder, S. H., \& Sabatini, D. M. (1998). RAFT1 phosphorylation of the translational regulators p70 S6 kinase and 4E-BP1. Proceedings of the National Academy of Sciences, 95(4), 1432-1437. https://doi.org/10.1073/pnas.95.4.1432

Buszczak, M., Signer, R. A. J., \& Morrison, S. J. (2014, October). Cellular differences in protein synthesis regulate tissue homeostasis. Cell. https://doi.org/10.1016/j.cell.2014.09.016

Bygrave, F. L., \& Benedetti, A. (1996). What is the concentration of calcium ions in the endoplasmic reticulum? Cell Calcium, 19(6), 547-551. https://doi.org/10.1016/S01434160(96)90064-0

Cai, X., Xu, Y., Cheung, A. K., Tomlinson, R. C., Alcázar-Román, A., Murphy, L., ... Huang, Q. (2013). PIKfyve, a Class III PI Kinase, Is the Target of the Small Molecular IL-12/IL-23 Inhibitor Apilimod and a Player in Toll-like Receptor Signaling. Chemistry \& Biology, 20(7), 912-921. https://doi.org/10.1016/j.chembiol.2013.05.010

Campos-Melo, D., Droppelmann, C. A., Volkening, K., \& Strong, M. J. (2014). Comprehensive luciferase-based reporter gene assay reveals previously masked up-regulatory effects of miRNAs. International Journal of Molecular Sciences. 
https://doi.org/10.3390/ijms150915592

Cañedo-Dorantes, L., \& Cañedo-Ayala, M. (2019). Skin Acute Wound Healing: A Comprehensive Review. International Journal of Inflammation, 2019, 1-15. https://doi.org/10.1155/2019/3706315

Cannon, G J, \& Swanson, J. A. (1992). The macrophage capacity for phagocytosis. Journal of Cell Science.

Cannon, Gregory J, \& Swanson, J. A. (1992). The macrophage capacity for phagocytosis. Journal of Cell Science, 101, 907-913.

Cantalupo, G., Alifano, P., Roberti, V., Bruni, C. B., \& Bucci, C. (2001). Rab-interacting lysosomal protein (RILP): the Rab7 effector required for transport to lysosomes. The EMBO Journal, 20(4), 683-693. https://doi.org/10.1093/emboj/20.4.683

Cao, C., Backer, J. M., Laporte, J., Bedrick, E. J., \& Wandinger-Ness, A. (2008). Sequential Actions of Myotubularin Lipid Phosphatases Regulate Endosomal PI(3)P and Growth Factor Receptor Trafficking. Molecular Biology of the Cell. https://doi.org/10.1091/mbc.e08-040367

Carlton, J. G., \& Cullen, P. J. (2005). Coincidence detection in phosphoinositide signaling. Trends in Cell Biology. https://doi.org/10.1016/j.tcb.2005.08.005

Carroll, B., \& Dunlop, E. A. (2017). The lysosome: a crucial hub for AMPK and mTORC1 signalling. Biochemical Journal. https://doi.org/10.1042/bcj20160780

Casey, J. R., Grinstein, S., \& Orlowski, J. (2010). Sensors and regulators of intracellular pH. Nature Reviews Molecular Cell Biology. https://doi.org/10.1038/nrm2820

Cella, M., Engering, A., Pinet, V., Pieters, J., \& Lanzavecchia, A. (1997). Inflammatory stimuli induce accumulation of MHC class II complexes on dendritic cells. Nature, 388(6644), 782787. https://doi.org/10.1038/42030

Champion, J. A., \& Mitragotri, S. (2006). Role of target geometry in phagocytosis. Proceedings of the National Academy of Sciences, 103(13), 4930-4934. https://doi.org/10.1073/pnas.0600997103

Champion, Julie A., \& Mitragotri, S. (2009). Shape induced inhibition of phagocytosis of polymer 
particles. Pharmaceutical Research, 26(1), 244-249. https://doi.org/10.1007/s11095-0089626-Z

Champion, Julie A, Walker, A., \& Mitragotri, S. (2008). Role of Particle Size in Phagocytosis of Polymeric Microspheres. Pharmaceutical Research, 25(8), 1815-1821. https://doi.org/10.1007/s11095-008-9562-y

Chan, Y.-H. M., Reyes, L., Sohail, S. M., Tran, N. K., \& Marshall, W. F. (2016). Organelle Size Scaling of the Budding Yeast Vacuole by Relative Growth and Inheritance. Current Biology, 26(9), 1221-1228. https://doi.org/10.1016/j.cub.2016.03.020

Chantranupong, L., Scaria, S. M., Saxton, R. A., Gygi, M. P., Shen, K., Wyant, G. A., ... Sabatini, D. M. (2016). The CASTOR Proteins Are Arginine Sensors for the mTORC1 Pathway. Cell, 165(1), 153-164. https://doi.org/10.1016/j.cell.2016.02.035

Chaplin, D. D. (2010). Overview of the immune response. The Journal of Allergy and Clinical Immunology, 125(2 Suppl 2), S3-23. https://doi.org/10.1016/j.jaci.2009.12.980

Chen, H., Vermulst, M., Wang, Y. E., Chomyn, A., Prolla, T. A., McCaffery, J. M., \& Chan, D. C. (2010). Mitochondrial Fusion Is Required for mtDNA Stability in Skeletal Muscle and Tolerance of mtDNA Mutations. Cell, 141(2), 280-289. https://doi.org/10.1016/j.cell.2010.02.026

Chow, A., Toomre, D., Garrett, W., \& Mellman, I. (2002). Dendritic cell maturation triggers retrograde MHC class II transport from lysosomes to the plasma membrane. Nature, 418(6901), 988-994. https://doi.org/10.1038/nature01006

Choy, C. H., Han, B.-K., \& Botelho, R. J. (2017). Phosphoinositide Diversity, Distribution, and Effector Function: Stepping Out of the Box. BioEssays, 39(12), 1700121. https://doi.org/10.1002/bies.201700121

Christensen, K. a, Myers, J. T., \& Swanson, J. a. (2002). pH-dependent regulation of lysosomal calcium in macrophages. Journal of Cell Science, 115, 599-607.

Christoforidis, S., McBride, H. M., Burgoyne, R. D., \& Zerial, M. (1999). The Rab5 effector EEA1 is a core component of endosome docking. Nature, 397(6720), 621-625. https://doi.org/10.1038/17618 
Christoforidis, S., Miaczynska, M., Ashman, K., Wilm, M., Zhao, L., Yip, S.-C., ... Zerial, M. (1999). Phosphatidylinositol-3-OH kinases are Rab5 effectors. Nature Cell Biology, 1(4), 249-252. https://doi.org/10.1038/12075

Coffey, J. W., \& De Duve, C. (1968). Digestive activity of lysosomes. I. The digestion of proteins by extracts of rat liver lysosomes. The Journal of Biological Chemistry, 243(12), 3255-3263.

Corno, G., \& Jürgens, K. (2006). Direct and indirect effects of protist predation on population size structure of a bacterial strain with high phenotypic plasticity. Applied and Environmental Microbiology, 72(1), 78-86. https://doi.org/10.1128/AEM.72.1.78

Corradin, S. B., Mauël, J., Gallay, P., Heumann, D., Ulevitch, R. J., \& Tobias, P. S. (1992). Enhancement of murine macrophage binding of and response to bacterial lipopolysaccharide (LPS) by LPS-binding protein. Journal of Leukocyte Biology, 52(4), 363-368.

Covarrubias, A. J., Aksoylar, H. I., \& Horng, T. (2015). Control of macrophage metabolism and activation by mTOR and Akt signaling. Seminars in Immunology, 27(4), 286-296. https://doi.org/10.1016/j.smim.2015.08.001

Dadaglio, G., Nelson, C. A., Deck, M. B., Petzold, S. J., \& Unanue, E. R. (1997). Characterization and Quantitation of Peptide-MHC Complexes Produced from Hen Egg Lysozyme Using a Monoclonal Antibody. Immunity, 6(6), 727-738. https://doi.org/10.1016/S10747613(00)80448-3

Dalvai, M., Loehr, J., Jacquet, K., Huard, C. C., Roques, C., Herst, P., ... Doyon, Y. (2015). A Scalable Genome-Editing-Based Approach for Mapping Multiprotein Complexes in Human Cells. Cell Reports. https://doi.org/10.1016/j.celrep.2015.09.009

Daniele, T., Hackmann, Y., Ritter, A. T., Wenham, M., Booth, S., Bossi, G., ... Griffiths, G. M. (2011). A Role for Rab7 in the Movement of Secretory Granules in Cytotoxic $\mathrm{T}$ Lymphocytes. Traffic, 12(7), 902-911. https://doi.org/10.1111/j.1600-0854.2011.01194.x

Dayam, R. M., Saric, A., Shilliday, R. E., \& Botelho, R. J. (2015). The Phosphoinositide-Gated Lysosomal Ca 2+ Channel, TRPML1, Is Required for Phagosome Maturation. Traffic, 16(9), 1010-1026. https://doi.org/10.1111/tra.12303

Dayam, R. M., Sun, C. X., Choy, C. H., Mancuso, G., Glogauer, M., \& Botelho, R. J. (2017). The 
Lipid Kinase PIKfyve Coordinates the Neutrophil Immune Response through the Activation of the Rac GTPase. The Journal of Immunology. https://doi.org/10.4049/jimmunol.1601466

Delamarre, L., Pack, M., Chang, H., Mellman, I., \& Trombetta, E. S. (2005). Differential lysosomal proteolysis in antigen-presenting cells determines antigen fate. Science (New York, N.Y.), 307(5715), 1630-1634. https://doi.org/10.1126/science.1108003

Dell'Angelica, E. C., Mullins, C., Caplan, S., \& Bonifacino, J. S. (2000). Lysosome-related organelles. FASEB Journal : Official Publication of the Federation of American Societies for Experimental Biology, 14(10), 1265-1278.

Demaurex, N. (2002). pH Homeostasis of cellular organelles. News in Physiological Sciences : An International Journal of Physiology Produced Jointly by the International Union of Physiological Sciences and the American Physiological Society.

Deretic, V., \& Levine, B. (2018). Autophagy balances inflammation in innate immunity. Autophagy, 14(2), 243-251. https://doi.org/10.1080/15548627.2017.1402992

Di Pasquale, A., Preiss, S., Tavares Da Silva, F., \& Garçon, N. (2015). Vaccine Adjuvants: from 1920 to 2015 and Beyond. Vaccines, 3(2), 320-343. https://doi.org/10.3390/vaccines3020320

Doherty, G. P., Bailey, K., \& Lewis, P. J. (2010). Stage-specific fluorescence intensity of GFP and mCherry during sporulation In Bacillus Subtilis. BMC Research Notes, 3(1), 303. https://doi.org/10.1186/1756-0500-3-303

Drutman, S. B., \& Trombetta, E. S. (2010). Dendritic Cells Continue To Capture and Present Antigens after Maturation In Vivo. The Journal of Immunology, 185(4), 2140-2146. https://doi.org/10.4049/jimmunol.1000642

Durchfort, N., Verhoef, S., Vaughn, M. B., Shrestha, R., Adam, D., Kaplan, J., \& Ward, D. M. (2012). The enlarged lysosomes in beige $\mathrm{j}$ cells result from decreased lysosome fission and not increased lysosome fusion. Traffic, 13, 108-119. https://doi.org/10.1111/j.16000854.2011.01300.x

Egner, A., Jakobs, S., \& Hell, S. W. (2002). Fast 100-nm resolution three-dimensional microscope reveals structural plasticity of mitochondria in live yeast. Proceedings of the National Academy of Sciences of the United States of America, 99(6), 3370-3375. 
https://doi.org/10.1073/pnas.052545099

Ellson, C. D., Anderson, K. E., Morgan, G., Chilvers, E. R., Lipp, P., Stephens, L. R., \& Hawkins, P. T. (2001). Phosphatidylinositol 3-phosphate is generated in phagosomal membranes. Current Biology, 11(20), 1631-1635. https://doi.org/10.1016/S0960-9822(01)00447-X

Erie, C., Sacino, M., Houle, L., Lu, M. L., \& Wei, J. (2015). Altered lysosomal positioning affects lysosomal functions in a cellular model of Huntington's disease. European Journal of Neuroscience, 42(3), 1941-1951. https://doi.org/10.1111/ejn.12957

Eskelinen, E.-L. (2006). Roles of LAMP-1 and LAMP-2 in lysosome biogenesis and autophagy. Molecular Aspects of Medicine, 27(5-6), 495-502. https://doi.org/10.1016/J.MAM.2006.08.005

Eskelinen, E. L., Tanaka, Y., \& Saftig, P. (2003). At the acidic edge: Emerging functions for lysosomal membrane proteins. Trends in Cell Biology, 13(3), 137-145. https://doi.org/10.1016/S0962-8924(03)00005-9

Ezaki, J., Himeno, M., \& Kato, K. (1992). Purification and characterization of (Ca2+-Mg2+)ATPase in rat liver lysosomal membranes. Journal of Biochemistry, 112(1), 33-39.

Fadden, P., Haystead, T. A. J., \& Lawrence, J. C. (1997). Identification of Phosphorylation Sites in the Translational Regulator, PHAS-I, That Are Controlled by Insulin and Rapamycin in Rat Adipocytes. Journal of Biological Chemistry, 272(15), 10240-10247. https://doi.org/10.1074/jbc.272.15.10240

Fairn, G. D., \& Grinstein, S. (2012). How nascent phagosomes mature to become phagolysosomes. Trends in Immunology. https://doi.org/10.1016/j.it.2012.03.003

Feng, Q., \& Kornmann, B. (2018). Mechanical forces on cellular organelles. Journal of Cell Science. https://doi.org/10.1242/jcs.218479

Fennelly, C., \& Amaravadi, R. K. (2017). Lysosomal Biology in Cancer. Methods in Molecular Biology (Clifton, N.J.), 1594, 293-308. https://doi.org/10.1007/978-1-4939-6934-0_19

Filipek, P. A., de Araujo, M. E. G., Vogel, G. F., De Smet, C. H., Eberharter, D., Rebsamen, M., ... Huber, L. A. (2017). LAMTOR/Ragulator is a negative regulator of Arl8b- and BORCdependent late endosomal positioning. The Journal of Cell Biology, 216(12), 4199-4215. 
https://doi.org/10.1083/jcb.201703061

Flannagan, R. S., Jaumouillé, V., \& Grinstein, S. (2012). The Cell Biology of Phagocytosis. Annual Review of Pathology: Mechanisms of Disease, 7(1), 61-98. https://doi.org/10.1146/annurev-pathol-011811-132445

Fleisher, T. A. (2012). Immune Dysregulation Disorders. In Textbook of Clinical Pediatrics (pp. 1307-1314). Berlin, Heidelberg: Springer Berlin Heidelberg. https://doi.org/10.1007/978-3642-02202-9_126

Fox, C. B., Kramer, R. M., Barnes V, L., Dowling, Q. M., \& Vedvick, T. S. (2014). Working together: interactions between vaccine antigens and adjuvants. Therapeutic Advances in Vaccines. https://doi.org/10.1177/2051013613480144

Gaard, E., Gislason, A., Falkenhaug, T., Søiland, H., Musaeva, E., Vereshchaka, A., \& Vinogradov, G. (2008). Horizontal and vertical copepod distribution and abundance on the Mid-Atlantic Ridge in June 2004. Deep-Sea Research Part II: Topical Studies in Oceanography, 55(1-2), 59-71. https://doi.org/10.1016/j.dsr2.2007.09.012

Gandin, V., Masvidal, L., Hulea, L., Gravel, S. P., Cargnello, M., McLaughlan, S., ... Topisirovic, I. (2016). NanoCAGE reveals 5' UTR features that define specific modes of translation of functionally related MTOR-sensitive mRNAs. Genome Research, 26(5), 636-648. https://doi.org/10.1101/gr.197566.115

Gandin, V., Sikström, K., Alain, T., Morita, M., McLaughlan, S., Larsson, O., \& Topisirovic, I. (2014). Polysome fractionation and analysis of mammalian translatomes on a genome-wide scale. Journal of Visualized Experiments : JoVE, (87). https://doi.org/10.3791/51455

Ganley, I. G., Lam, D. H., Wang, J., Ding, X., Chen, S., \& Jiang, X. (2009). ULK1·ATG13·FIP200 complex mediates mTOR signaling and is essential for autophagy. Journal of Biological Chemistry, 284(18), 12297-12305. https://doi.org/10.1074/jbc.M900573200

Gantner, B. N., Simmons, R. M., Canavera, S. J., Akira, S., \& Underhill, D. M. (2003). Collaborative Induction of Inflammatory Responses by Dectin-1 and Toll-like Receptor 2. The Journal of Experimental Medicine, 197(9), 1107-1117. https://doi.org/10.1084/jem.20021787 
Garami, A., Zwartkruis, F. J. ., Nobukuni, T., Joaquin, M., Roccio, M., Stocker, H., ... Thomas, G. (2003). Insulin Activation of Rheb, a Mediator of mTOR/S6K/4E-BP Signaling, Is Inhibited by TSC1 and 2. Molecular Cell, 11(6), 1457-1466. https://doi.org/10.1016/S10972765(03)00220-X

Garçon, N., Morel, S., Didierlaurent, A., Descamps, D., Wettendorff, M., \& Van Mechelen, M. (2011). Development of an AS04-Adjuvanted HPV Vaccine with the Adjuvant System Approach. BioDrugs, 25(4), 217-226. https://doi.org/10.2165/11591760-000000000-00000

Garg, S., Sharma, M., Ung, C., Tuli, A., Barral, D. C., Hava, D. L., .. Brenner, M. B. (2011). Lysosomal Trafficking, Antigen Presentation, and Microbial Killing Are Controlled by the Arf-like GTPase Arl8b. Immunity. https://doi.org/10.1016/j.immuni.2011.06.009

Garrett, W. S., Chen, L. M., Kroschewski, R., Ebersold, M., Turley, S., Trombetta, S., ... Mellman, I. (2000). Developmental control of endocytosis in dendritic cells by Cdc42. Cell. https://doi.org/10.1016/S0092-8674(00)00038-6

Gary, J. D., Wurmser, A. E., Bonangelino, C. J., Weisman, L. S., \& Emr, S. D. (1998). Fab1p is essential for PtdIns(3)P 5-kinase activity and the maintenance of vacuolar size and membrane homeostasis. The Journal of Cell Biology, 143(1), 65-79.

Gay, N. J., Symmons, M. F., Gangloff, M., \& Bryant, C. E. (2014). Assembly and localization of Toll-like receptor signalling complexes. Nature Reviews Immunology, 14(8), 546-558. https://doi.org/10.1038/nri3713

Genc, G. E., Hipolito, V. E. B., Botelho, R. J., \& Gumuslu, S. (2018). Lysophosphatidic acid represses autophagy in prostate carcinoma cells. Biochemistry and Cell Biology, bcb-20180164. https://doi.org/10.1139/bcb-2018-0164

Gerisch, G., Ecke, M., Schroth-Diez, B., Gerwig, S., Engel, U., Maddera, L., \& Clarke, M. (2009). Self-organizing actin waves as planar phagocytic cup structures. Cell Adhesion and Migration. https://doi.org/10.4161/cam.3.4.9708

Gharbi, S. I., Zvelebil, M. J., Shuttleworth, S. J., Hancox, T., Saghir, N., Timms, J. F., \& Waterfield, M. D. (2007). Exploring the specificity of the PI3K family inhibitor LY294002. Biochemical Journal. https://doi.org/10.1042/bj20061489 
Gillooly, D. J., Morrow, I. C., Lindsay, M., Gould, R., Bryant, N. J., Gaullier, J. M., ... Stenmark, H. (2000). Localization of phosphatidylinositol 3-phosphate in yeast and mammalian cells. The EMBO Journal, 19(17), 4577-4588. https://doi.org/10.1093/emboj/19.17.4577

Gingras, A. C., Gygi, S. P., Raught, B., Polakiewicz, R. D., Abraham, R. T., Hoekstra, M. F., ... Sonenberg, N. (1999). Regulation of 4E-BP1 phosphorylation: a novel two-step mechanism. Genes \& Development, 13(11), 1422-1437.

Golebiewska, U., Kay, J. G., Masters, T., Grinstein, S., Im, W., Pastor, R. W., .. McLaughlin, S. (2011). Evidence for a fence that impedes the diffusion of phosphatidylinositol 4,5bisphosphate out of the forming phagosomes of macrophages. Molecular Biology of the Cell. https://doi.org/10.1091/mbc.e11-02-0114

Gomes, E., \& Shorter, J. (2018). The molecular language of membraneless organelles. Journal of Biological Chemistry, jbc.TM118.001192. https://doi.org/10.1074/jbc.TM118.001192

Graves, A. R., Curran, P. K., Smith, C. L., \& Mindell, J. A. (2008). The Cl-/H+ antiporter ClC-7 is the primary chloride permeation pathway in lysosomes. Nature, 453(7196), 788-792. https://doi.org/10.1038/nature06907

Gray, M. A., Choy, C. H., Dayam, R. M., Ospina-Escobar, E., Somerville, A., Xiao, X., ... Botelho, R. J. (2016). Phagocytosis Enhances Lysosomal and Bactericidal Properties by Activating the Transcription Factor TFEB. Current Biology, 26(15), 1955-1964. https://doi.org/10.1016/j.cub.2016.05.070

Gray, M., \& Botelho, R. J. (2017). Phagocytosis: Hungry, hungry cells. In Methods in Molecular Biology (Vol. 1519, pp. 1-16). https://doi.org/10.1007/978-1-4939-6581-6_1

Grosshans, B. L., Ortiz, D., \& Novick, P. (2006). Rabs and their effectors: achieving specificity in membrane traffic. Proceedings of the National Academy of Sciences of the United States of America, 103(32), 11821-11827. https://doi.org/10.1073/pnas.0601617103

Guardia, C. M., Farías, G. G., Jia, R., Pu, J., \& Bonifacino, J. S. (2016). BORC Functions Upstream of Kinesins 1 and 3 to Coordinate Regional Movement of Lysosomes along Different Microtubule Tracks. Cell Reports, 17(8), 1950-1961. https://doi.org/10.1016/j.celrep.2016.10.062 
Guertin, D. A., \& Sabatini, D. M. (2007). Defining the Role of mTOR in Cancer. Cancer Cell, 12(1), 9-22. https://doi.org/10.1016/j.ccr.2007.05.008

Guo, H., Callaway, J. B., \& Ting, J. P. Y. (2015). Inflammasomes: mechanism of action, role in disease and therapeutics. Nature Medicine, 21(7), 677-687. https://doi.org/10.1038/nm.3893

Gustafsson, M. G. L. (2005). Nonlinear structured-illumination microscopy: Wide-field fluorescence imaging with theoretically unlimited resolution. Proceedings of the National Academy of Sciences, 102(37), 13081-13086. https://doi.org/10.1073/pnas.0406877102

Gutjahr, A., Tiraby, G., Perouzel, E., Verrier, B., \& Paul, S. (2016). Triggering Intracellular Receptors for Vaccine Adjuvantation. Trends in Immunology, 37(9), 573-587. https://doi.org/10.1016/j.it.2016.07.001

Gwinn, D. M., Shackelford, D. B., Egan, D. F., Mihaylova, M. M., Mery, A., Vasquez, D. S., ... Shaw, R. J. (2008). AMPK Phosphorylation of Raptor Mediates a Metabolic Checkpoint. Molecular Cell, 30(2), 214-226. https://doi.org/10.1016/j.molcel.2008.03.003

Hammond, G. R. V., Machner, M. P., \& Balla, T. (2014). A novel probe for phosphatidylinositol 4-phosphate reveals multiple pools beyond the Golgi. The Journal of Cell Biology, 205(1), 113-126. https://doi.org/10.1083/jcb.201312072

Hao, X., Wang, Y., Ren, F., Zhu, S., Ren, Y., Jia, B., ... Chang, Z. (2011). SNX25 regulates TGF$\beta$ signaling by enhancing the receptor degradation. Cellular Signalling. https://doi.org/10.1016/j.cellsig.2011.01.022

Harding, C., Heuser, J., \& Stahl, P. (1983). Receptor-mediated endocytosis of transferrin and recycling of the transferrin receptor in rat reticulocytes. The Journal of Cell Biology, 97(2), 329-339. https://doi.org/10.1083/jcb.97.2.329

Harrison, R. E., Bucci, C., Vieira, O. V., Schroer, T. A., \& Grinstein, S. (2003). Phagosomes fuse with late endosomes and/or lysosomes by extension of membrane protrusions along microtubules: role of Rab7 and RILP. Molecular and Cellular Biology, 23(18), 6494-6506. https://doi.org/10.1128/MCB.23.18.6494-6506.2003

Haupts, U., Maiti, S., Schwille, P., \& Webb, W. W. (1998). Dynamics of fluorescence fluctuations in green fluorescent protein observed by fluorescence correlation spectroscopy. Proceedings 
of the National Academy of Sciences, 95(23), 13573-13578. https://doi.org/10.1073/pnas.95.23.13573

Hazeki, K., Nigorikawa, K., Takaba, Y., Segawa, T., Nukuda, A., Masuda, A., ... Hazeki, O. (2012). Essential roles of PIKfyve and PTEN on phagosomal phosphatidylinositol 3phosphate dynamics. FEBS Letters, 586(22), 4010-4015. https://doi.org/10.1016/j.febslet.2012.09.043

He, J., Vora, M., Haney, R. M., Filonov, G. S., Musselman, C. A., Burd, C. G., ... Kutateladze, T. G. (2009). Membrane insertion of the FYVE domain is modulated by pH. Proteins: Structure, Function and Bioinformatics. https://doi.org/10.1002/prot.22392

Heinsbroek, S. E. M., Kamen, L. A., Taylor, P. R., Brown, G. D., Swanson, J., \& Gordon, S. (2009). Actin and Phosphoinositide Recruitment to Fully Formed Candida albicans Phagosomes in Mouse Macrophages. Journal of Innate Immunity, 1(3), 244-253. https://doi.org/10.1159/000173694

Herre, J., Marshall, A. S. J., Caron, E., Edwards, A. D., Williams, D. L., Schweighoffer, E., ... Brown, G. D. (2004). Dectin-1 uses novel mechanisms for yeast phagocytosis in macrophages. Blood, 104(13), 4038-4045. https://doi.org/10.1182/blood-2004-03-1140

Heuser, J. (1989). Changes in lysosome shape and distribution correlated with changes in cytoplasmic pH. Journal of Cell Biology, 108(3), 855-864. https://doi.org/10.1083/jcb.108.3.855

Hipolito, V. E. B., Ospina-Escobar, E., \& Botelho, R. J. (2018). Lysosome remodelling and adaptation during phagocyte activation. Cellular Microbiology, 20(4), e12824. https://doi.org/10.1111/cmi.12824

Ho, C. Y., Alghamdi, T. A., \& Botelho, R. J. (2012). Phosphatidylinositol-3,5-bisphosphate: no longer the poor PIP2. Traffic (Copenhagen, Denmark), 13(1), 1-8. https://doi.org/10.1111/j.1600-0854.2011.01246.x

Ho, C. Y., Choy, C. H., \& Botelho, R. J. (2016). Radiolabeling and Quantification of Cellular Levels of Phosphoinositides by High Performance Liquid Chromatography-coupled Flow Scintillation. Journal of Visualized Experiments. https://doi.org/10.3791/53529 
Hofmann, I. (2006). An N-terminally acetylated Arf-like GTPase is localised to lysosomes and affects their motility. Journal of Cell Science, 119(8), 1494-1503. https://doi.org/10.1242/jcs.02958

Hollenbeck, P. J., \& Swanson, J. a. (1990). Radial extension of macrophage tubular lysosomes supported by kinesin. Nature, 346, 864-866. https://doi.org/10.1038/346864a0

Holz, M. K., Ballif, B. A., Gygi, S. P., \& Blenis, J. (2005). mTOR and S6K1 mediate assembly of the translation preinitiation complex through dynamic protein interchange and ordered phosphorylation events. Cell, 123(4), 569-580. https://doi.org/10.1016/j.cell.2005.10.024

Horiuchi, H., Lippé, R., McBride, H. M., Rubino, M., Woodman, P., Stenmark, H., ... Zerial, M. (1997). A novel Rab5 GDP/GTP exchange factor complexed to Rabaptin-5 links nucleotide exchange to effector recruitment and function. Cell, 90(6), 1149-1159. https://doi.org/10.1016/S0092-8674(00)80380-3

Horvath, D. J., Li, B., Casper, T., Partida-Sanchez, S., Hunstad, D. A., Hultgren, S. J., \& Justice, S. S. (2011). Morphological plasticity promotes resistance to phagocyte killing of uropathogenic Escherichia coli. Microbes and Infection, 13(5), 426-437. https://doi.org/10.1016/j.micinf.2010.12.004

Hosokawa, N., Hara, T., Kaizuka, T., Kishi, C., Takamura, A., Miura, Y., ... Mizushima, N. (2009). Nutrient-dependent mTORC1 association with the ULK1-Atg13-FIP200 complex required for autophagy. Molecular Biology of the Cell, 20(7), 1981-1991.

Hsieh, A. C., Costa, M., Zollo, O., Davis, C., Feldman, M. E., Testa, J. R., ... Ruggero, D. (2010). Genetic Dissection of the Oncogenic mTOR Pathway Reveals Druggable Addiction to Translational Control via 4EBP-eIF4E. Cancer Cell, 17(3), 249-261. https://doi.org/10.1016/j.ccr.2010.01.021

Hsieh, A. C., Liu, Y., Edlind, M. P., Ingolia, N. T., Janes, M. R., Sher, A., ... Ruggero, D. (2012). The translational landscape of mTOR signalling steers cancer initiation and metastasis. Nature, 485(7396), 55-61. https://doi.org/10.1038/nature10912

Huang, N.-N., Becker, S., Boularan, C., Kamenyeva, O., Vural, A., Hwang, I.-Y., ... Kehrl, J. H. (2014). Canonical and Noncanonical G-Protein Signaling Helps Coordinate Actin Dynamics To Promote Macrophage Phagocytosis of Zymosan. Molecular and Cellular Biology, 34(22), 
4186-4199. https://doi.org/10.1128/MCB.00325-14

Huffman, T. A., Mothe-Satney, I., \& Lawrence, J. C. (2002). Insulin-stimulated phosphorylation of lipin mediated by the mammalian target of rapamycin. Proceedings of the National Academy of Sciences, 99(2), 1047-1052. https://doi.org/10.1073/pnas.022634399

Hunziker, W., Simmen, T., \& Höning, S. (1996). Trafficking of lysosomal membrane proteins in polarized kidney cells. Nephrologie, 17(7), 347-350.

Hurtado-Lorenzo, A., Skinner, M., Annan, J. El, Futai, M., Sun-Wada, G.-H., Bourgoin, S., ... Marshansky, V. (2006). V-ATPase interacts with ARNO and Arf6 in early endosomes and regulates the protein degradative pathway. Nature Cell Biology, 8(2), 124-136. https://doi.org/10.1038/ncb1348

Huss, M., Ingenhorst, G., König, S., Gaßel, M., Dröse, S., Zeeck, A., .. Wieczorek, H. (2002). Concanamycin A, the Specific Inhibitor of V-ATPases, Binds to the V o Subunit c. Journal of Biological Chemistry, 277(43), 40544-40548. https://doi.org/10.1074/jbc.M207345200

Inaba, K., Inaba, M., Romani, N., Aya, H., Deguchi, M., Ikehara, S., .. Steinman, R. M. (1992). Generation of large numbers of dendritic cells from mouse bone marrow cultures supplemented with granulocyte/macrophage colony-stimulating factor. The Journal of Experimental Medicine, 176(6), 1693-1702. https://doi.org/10.1084/jem.176.6.1693

Inoki, K., Li, Y., Zhu, T., Wu, J., \& Guan, K. (2002). TSC2 is phosphorylated and inhibited by Akt and suppresses mTOR signalling. Nature Cell Biology, 4(9), 648-657. https://doi.org/10.1038/ncb839

Inoki, K., Zhu, T., \& Guan, K.-L. (2003). TSC2 mediates cellular energy response to control cell growth and survival. Cell, 115(5), 577-590. https://doi.org/10.1016/S0092-8674(03)00929-2

Inpanathan, S., \& Botelho, R. J. (2019). The Lysosome Signaling Platform: Adapting With the Times. Frontiers in Cell and Developmental Biology, 7(June), 1-22. https://doi.org/10.3389/fcell.2019.00113

Ishida, Y., Nayak, S., Mindell, J. A., \& Grabe, M. (2013). A model of lysosomal pH regulation. The Journal of General Physiology, 141(6), 705-720. https://doi.org/10.1085/jgp.201210930

Itoh, K., Adachi, Y., Yamada, T., Suzuki, T. L., Otomo, T., McBride, H. M., ... Sesaki, H. (2018). 
A brain-enriched Drp1 isoform associates with lysosomes, late endosomes, and the plasma membrane. Journal of Biological Chemistry, 293(30), 11809-11822. https://doi.org/10.1074/jbc.RA117.001253

Itoh, T., Koshiba, S., Kigawa, T., Kikuchi, A., Yokoyama, S., \& Takenawa, T. (2001). Role of the ENTH domain in phosphatidylinositol-4,5-bisphosphate binding and endocytosis. Science, 291(5506), 1047-1051. https://doi.org/10.1126/science.291.5506.1047

Iwasaki, A., \& Medzhitov, R. (2015). Control of adaptive immunity by the innate immune system. Nature Immunology, 16(4), 343-353. https://doi.org/10.1038/ni.3123

Jancic, C., Savina, A., Wasmeier, C., Tolmachova, T., El-Benna, J., Dang, P. M.-C., ... Amigorena, S. (2007). Rab27a regulates phagosomal pH and NADPH oxidase recruitment to dendritic cell phagosomes. Nature Cell Biology, 9(4), 367-378. https://doi.org/10.1038/ncb1552

Janssens, S., Pulendran, B., \& Lambrecht, B. N. (2014). Emerging functions of the unfolded protein response in immunity. Nature Immunology, 15(10), 910-919. https://doi.org/10.1038/ni.2991

Jastrzebski, K., Hannan, K. M., Tchoubrieva, E. B., Hannan, R. D., \& Pearson, R. B. (2007). Coordinate regulation of ribosome biogenesis and function by the ribosomal protein S6 kinase, a key mediator of mTOR function. Growth Factors, 25(4), 209-226. https://doi.org/10.1080/08977190701779101

Jefferies, H. B., Reinhard, C., Kozma, S. C., \& Thomas, G. (1994). Rapamycin selectively represses translation of the "polypyrimidine tract" mRNA family. Proceedings of the National Academy of Sciences of the United States of America, 91(10), 4441-4445.

Jensen, S. S., Aaberg-Jessen, C., Christensen, K. G., \& Kristensen, B. (2013). Expression of the lysosomal-associated membrane protein-1 (LAMP-1) in astrocytomas. International Journal of Clinical and Experimental Pathology, 6(7), 1294-1305.

Jewell, J. L., Russell, R. C., \& Guan, K.-L. (2013). Amino acid signalling upstream of mTOR. Nature Reviews. Molecular Cell Biology, 14(3), 133-139. https://doi.org/10.1038/nrm3522

Jin, N., Lang, M. J., \& Weisman, L. S. (2016). Phosphatidylinositol 3,5-bisphosphate: regulation 
of cellular events in space and time. Biochemical Society Transactions. https://doi.org/10.1042/bst20150174

Johansson, M., Rocha, N., Zwart, W., Jordens, I., Janssen, L., Kuijl, C., ... Neefjes, J. (2007). Activation of endosomal dynein motors by stepwise assembly of Rab7-RILP-p150Glued, ORP1L, and the receptor BIII spectrin. Journal of Cell Biology. https://doi.org/10.1083/jcb.200606077

Johnson, A. E., Shu, H., Hauswirth, A. G., Tong, A., \& Davis, G. W. (2015). VCP-dependent muscle degeneration is linked to defects in a dynamic tubular lysosomal network in vivo. ELife, 4(JULY2015), 1-20. https://doi.org/10.7554/eLife.07366

Johnson, D. E., \& Casey, J. R. (2011). Cytosolic H + microdomain developed around AE1 during AE1-mediated Cl - /HCO 3 - exchange. The Journal of Physiology, 589(7), 1551-1569. https://doi.org/10.1113/jphysiol.2010.201483

Johnson, D. E., Ostrowski, P., Jaumouillé, V., \& Grinstein, S. (2016). The position of lysosomes within the cell determines their luminal pH. The Journal of Cell Biology, 212(6), 677-692. https://doi.org/10.1083/jcb.201507112

Jongsma, M. L. M., Berlin, I., Wijdeven, R. H. M., Janssen, L., Janssen, G. M. C., Garstka, M. A., ... Neefjes, J. (2016). An ER-Associated Pathway Defines Endosomal Architecture for Controlled Cargo Transport. Cell, 166(1), 152-166. https://doi.org/10.1016/j.cell.2016.05.078

Jordens, I., Fernandez-Borja, M., Marsman, M., Dusseljee, S., Janssen, L., Calafat, J., ... Neefjes, J. (2001). The Rab7 effector protein RILP controls lysosomal transport by inducing the recruitment of dynein-dynactin motors. Current Biology, 11(21), 1680-1685. https://doi.org/10.1016/S0960-9822(01)00531-0

Jung, C. H., Jun, C. B., Ro, S.-H., Kim, Y.-M., Otto, N. M., Cao, J., .. Kim, D.-H. (2009). ULKAtg13-FIP200 Complexes Mediate mTOR Signaling to the Autophagy Machinery. Molecular Biology of the Cell, 20(7), 1992-2003. https://doi.org/10.1091/mbc.E08-12-1249

Justice, S. S., Hunstad, D. A., Seed, P. C., \& Hultgren, S. J. (2006). Filamentation by Escherichia coli subverts innate defenses during urinary tract infection. Proceedings of the National Academy of Sciences, 103(52), 19884-19889. https://doi.org/10.1073/pnas.0606329104 
Justice, Sheryl S., Hunstad, D. A., Cegelski, L., \& Hultgren, S. J. (2008). Morphological plasticity as a bacterial survival strategy. Nature Reviews Microbiology. https://doi.org/10.1038/nrmicro1820

Kahvejian, A., Svitkin, Y. V, Sukarieh, R., M'Boutchou, M.-N., \& Sonenberg, N. (2005). Mammalian poly(A)-binding protein is a eukaryotic translation initiation factor, which acts via multiple mechanisms. Genes \& Development, 19(1), 104-113. https://doi.org/10.1101/gad.1262905

Kalani, M. R., Moradi, A., Moradi, M., \& Tajkhorshid, E. (2013). Characterizing a histidine switch controlling ph-dependent conformational changes of the influenza virus hemagglutinin. Biophysical Journal. https://doi.org/10.1016/j.bpj.2013.06.047

Kanai, F., Liu, H., Field, S. J., Akbary, H., Matsuo, T., Brown, G. E., ... Yaffe, M. B. (2001). The PX domains of p47phox and p40phox bind to lipid products of PI(3)K. Nature Cell Biology, 3(7), 675-678. https://doi.org/10.1038/35083070

Katzmann, D. J., Babst, M., \& Emr, S. D. (2001). Ubiquitin-dependent sorting into the multivesicular body pathway requires the function of a conserved endosomal protein sorting complex, ESCRT-I. Cell, 106(2), 145-155. https://doi.org/10.1016/S0092-8674(01)00434-2

Kelly, B., \& O’Neill, L. A. (2015). Metabolic reprogramming in macrophages and dendritic cells in innate immunity. Cell Research, 25(7), 771-784. https://doi.org/10.1038/cr.2015.68

Kim, D.-H., Sarbassov, D. D., Ali, S. M., Latek, R. R., Guntur, K. V. P., Erdjument-Bromage, H., ... Sabatini, D. M. (2003). G $\beta$ L, a Positive Regulator of the Rapamycin-Sensitive Pathway Required for the Nutrient-Sensitive Interaction between Raptor and mTOR. Molecular Cell, 11(4), 895-904. https://doi.org/10.1016/S1097-2765(03)00114-X

Kim, G. H. E., Dayam, R. M., Prashar, A., Terebiznik, M., \& Botelho, R. J. (2014). PIKfyve inhibition interferes with phagosome and endosome maturation in macrophages. Traffic. https://doi.org/10.1111/tra.12199

Kim, J. E., \& Chen, J. (2004). Regulation of peroxisome proliferator-activated receptor-gamma activity by mammalian target of rapamycin and amino acids in adipogenesis. Diabetes, 53(11), 2748-2756. 
Kinchen, J. M., \& Ravichandran, K. S. (2010). Identification of two evolutionarily conserved genes regulating processing of engulfed apoptotic cells. Nature, 464(7289), 778-782. https://doi.org/10.1038/nature08853

Kinoshita, E., Kinoshita-Kikuta, E., Takiyama, K., \& Koike, T. (2006). Phosphate-binding Tag, a New Tool to Visualize Phosphorylated Proteins. Molecular \& Cellular Proteomics. https://doi.org/10.1074/mcp.T500024-MCP200

Kinoshita, E., Takahashi, M., Takeda, H., Shiro, M., \& Koike, T. (2004). Recognition of phosphate monoester dianion by an alkoxide-bridged dinuclear zinc(ii) complex. Journal of the Chemical Society. Dalton Transactions. https://doi.org/10.1039/b400269e

Kirk, S. J., Cliff, J. M., Thomas, J. A., \& Ward, T. H. (2010). Biogenesis of secretory organelles during B cell differentiation. Journal of Leukocyte Biology, 87(February), 245-255. https://doi.org/10.1189/jlb.1208774

Kleijmeer, M., Ramm, G., Schuurhuis, D., Griffith, J., Rescigno, M., Ricciardi-Castagnoli, P., ... Geuze, H. J. (2001). Reorganization of multivesicular bodies regulates MHC class II 11 antigen presentation by dendritic cells. Journal of Cell Biology, 155(1), 53-63. https://doi.org/10.1083/jcb.200103071

Kleveta, G., Borzęcka, K., Zdioruk, M., Czerkies, M., Kuberczyk, H., Sybirna, N., ... Kwiatkowska, K. (2012). LPS induces phosphorylation of actin-regulatory proteins leading to actin reassembly and macrophage motility. Journal of Cellular Biochemistry, 113(1), 8092. https://doi.org/10.1002/jcb.23330

Koivusalo, M., Welch, C., Hayashi, H., Scott, C. C., Kim, M., Alexander, T., ... Grinstein, S. (2010). Amiloride inhibits macropinocytosis by lowering submembranous $\mathrm{pH}$ and preventing Rac1 and Cdc42 signaling. Journal of Cell Biology. https://doi.org/10.1083/jcb.200908086

Kong, D., \& Yamori, T. (2007). ZSTK474 is an ATP-competitive inhibitor of class I phosphatidylinositol 3 kinase isoforms. Cancer Science. https://doi.org/10.1111/j.13497006.2007.00580.x

Korolchuk, V. I., Saiki, S., Lichtenberg, M., Siddiqi, F. H., Roberts, E. A., Imarisio, S., ... Rubinsztein, D. C. (2011). Lysosomal positioning coordinates cellular nutrient responses. Nature Cell Biology, 13(4), 453-460. https://doi.org/10.1038/ncb2204 
Koromilas, A. E., \& Sonenberg, N. (1992). mRNAs containing extensive secondary structure in their 5 ' non-coding region translate efficiently in cells overexpressing initiation factor elF4E, 1(1), 4153-4158. https://doi.org/10.1002/j.1460-2075.1992.tb05508.x

Kraft, C., Kijanska, M., Kalie, E., Siergiejuk, E., Lee, S. S., Semplicio, G., ... Peter, M. (2012). Binding of the Atg1/ULK1 kinase to the ubiquitin-like protein Atg8 regulates autophagy. The EMBO Journal, 31(18), 3691-3703. https://doi.org/10.1038/emboj.2012.225

Krämer, L., \& Ungermann, C. (2011). HOPS drives vacuole fusion by binding the vacuolar SNARE complex and the Vam7 PX domain via two distinct sites. Molecular Biology of the Cell, 22(14), 2601-2611. https://doi.org/10.1091/mbc.e11-02-0104

Kropshofer, H., Arndt, S. O., Moldenhauer, G., Hämmerling, G. J., \& Vogt, A. B. (1997). HLA$\mathrm{DM}$ acts as a molecular chaperone and rescues empty HLA-DR molecules at lysosomal pH. Immunity, 6(3), 293-302. https://doi.org/10.1016/S1074-7613(00)80332-5

Lacombe, J., Karsenty, G., \& Ferron, M. (2013). Regulation of lysosome biogenesis and functions in osteoclasts. Cell Cycle (Georgetown, Tex.), 12(17), 2744-2752. https://doi.org/10.4161/cc.25825

Lacy, P. (2006). Mechanisms of Degranulation in Neutrophils. Allergy, Asthma \& Clinical Immunology, 2(3), 98. https://doi.org/10.1186/1710-1492-2-3-98

Lam, J., Herant, M., Dembo, M., \& Heinrich, V. (2009). Baseline Mechanical Characterization of J774 Macrophages. Biophysical Journal, 96(1), 248-254. https://doi.org/10.1529/biophysj.108.139154

Lancaster, C. E., Ho, C. Y., Hipolito, V. E. B., Botelho, R. J., \& Terebiznik, M. R. (2019). Phagocytosis: what's on the menu? Biochemistry and Cell Biology, 97(1), 21-29. https://doi.org/10.1139/bcb-2018-0008

Land, S. C., \& Tee, A. R. (2007). Hypoxia-inducible Factor $1 \alpha$ Is Regulated by the Mammalian Target of Rapamycin (mTOR) via an mTOR Signaling Motif. Journal of Biological Chemistry, 282(28), 20534-20543. https://doi.org/10.1074/jbc.M611782200

Laplante, M., \& Sabatini, D. M. (2009). mTOR signaling at a glance. Journal of Cell Science, 122(20), 3589-3594. https://doi.org/10.1242/jcs.051011 
Larsson, O., Morita, M., Topisirovic, I., Alain, T., Blouin, M.-J., Pollak, M., \& Sonenberg, N. (2012). Distinct perturbation of the translatome by the antidiabetic drug metformin. Proceedings of the National Academy of Sciences, 109(23), 8977-8982. https://doi.org/10.1073/pnas.1201689109

Lawe, D. C., Chawla, A., Merithew, E., Dumas, J., Carrington, W., Fogarty, K., ... Corvera, S. (2002). Sequential roles for phosphatidylinositol 3-phosphate and Rab5 in tethering and fusion of early endosomes via their interaction with EEA1. Journal of Biological Chemistry. https://doi.org/10.1074/jbc.M109239200

Lee, D., Kuo, H., Chen, C., Hsu, J., Chou, C., Wei, Y., ... Hung, M.-C. (2007). IKK $\beta$ Suppression of TSC1 Links Inflammation and Tumor Angiogenesis via the mTOR Pathway. Cell, 130(3), 440-455. https://doi.org/10.1016/j.cell.2007.05.058

Lee, S. A., Eyeson, R., Cheever, M. L., Geng, J., Verkhusha, V. V., Burd, C., ... Kutateladze, T. G. (2005). Targeting of the FYVE domain to endosomal membranes is regulated by a histidine switch. Proceedings of the National Academy of Sciences, 102(37), 13052-13057. https://doi.org/10.1073/pnas.0503900102

Legendre-Guillemin, V., Wasiak, S., Hussain, N. K., Angers, A., \& McPherson, P. S. (2004). ENTH/ANTH proteins and clathrin-mediated membrane budding. Journal of Cell Science, 117(Pt 1), 9-18. https://doi.org/10.1242/jcs.00928

Lehman, H. K. (2015). Autoimmunity and Immune Dysregulation in Primary Immune Deficiency Disorders. Current Allergy and Asthma Reports, 15(9), 53. https://doi.org/10.1007/s11882015-0553-x

Lei, N., Franken, L., Ruzehaji, N., Offenhauser, C., Cowin, A. J., \& Murray, R. Z. (2012). Flightless, secreted through a late endosome/lysosome pathway, binds LPS and dampens cytokine secretion. Journal of Cell Science, 125(18), 4288-4296. https://doi.org/10.1242/jcs.099507

Levin, R., Grinstein, S., \& Canton, J. (2016, September). The life cycle of phagosomes: formation, maturation, and resolution. Immunological Reviews. https://doi.org/10.1111/imr.12439

Levin, R., Grinstein, S., \& Schlam, D. (2015). Phosphoinositides in phagocytosis and macropinocytosis. Biochimica et Biophysica Acta - Molecular and Cell Biology of Lipids. 
https://doi.org/10.1016/j.bbalip.2014.09.005

Levy, D. L., \& Heald, R. (2012). Mechanisms of Intracellular Scaling. Annual Review of Cell and Developmental Biology, 28(1), 113-135. https://doi.org/10.1146/annurev-cellbio-092910154158

Li, J., Yin, H. L., \& Yuan, J. (2008). Flightless-I regulates proinflammatory caspases by selectively modulating intracellular localization and caspase activity. The Journal of Cell Biology, 181(2), 321-333. https://doi.org/10.1083/jcb.200711082

Li, X., Rydzewski, N., Hider, A., Zhang, X., Yang, J., Wang, W., ... Xu, H. (2016). A molecular mechanism to regulate lysosome motility for lysosome positioning and tubulation. Nature Cell Biology, 18(4), 404-417. https://doi.org/10.1038/ncb3324

Lim, C. Y., \& Zoncu, R. (2016). The lysosome as a command-and-control center for cellular metabolism. Journal of Cell Biology, 214(6), 653-664. https://doi.org/10.1083/jcb.201607005

Lin, S. Y., Hsu, W. H., Lo, J. M., Tsai, H. C., \& Hsiue, G. H. (2011). Novel geometry type of nanocarriers mitigated the phagocytosis for drug delivery. Journal of Controlled Release, 154(1), 84-92. https://doi.org/10.1016/j.jconrel.2011.04.023

Lippé, R., Miaczynska, M., Rybin, V., Runge, A., \& Zerial, M. (2001). Functional synergy between Rab5 effector Rabaptin-5 and exchange factor Rabex -5 when physically associated in a complex. Molecular Biology of the Cell, 12(7), 2219-2228. https://doi.org/10.1091/mbc.12.7.2219

Lloyd-Evans, E., \& Platt, F. M. (2011). Lysosomal $\mathrm{Ca}(2+)$ homeostasis: Role in pathogenesis of lysosomal storage diseases. Cell Calcium, 50(2), 200-205. https://doi.org/10.1016/j.ceca.2011.03.010

Long, X., Lin, Y., Ortiz-Vega, S., Yonezawa, K., \& Avruch, J. (2005). Rheb Binds and Regulates the mTOR Kinase. Current Biology, 15(8), 702-713. https://doi.org/10.1016/j.cub.2005.02.053

Lukacs, G. L., Rotstein, O. D., \& Grinstein, S. (1990). Phagosomal acidification is mediated by a vacuolar-type $\mathrm{H}(+)$-ATPase in murine macrophages. The Journal of Biological Chemistry, 
265(34), 21099-21107.

Lullmann-Rauch, R. (2005). History and morphology of the lysosome. Lysosomes, (July), 1-16.

Ma, X. M., Yoon, S.-O., Richardson, C. J., Jülich, K., \& Blenis, J. (2008). SKAR Links Pre-mRNA Splicing to mTOR/S6K1-Mediated Enhanced Translation Efficiency of Spliced mRNAs. Cell, 133(2), 303-313. https://doi.org/10.1016/j.cell.2008.02.031

Mantegazza, A. R., Savina, A., Vermeulen, M., Pérez, L., Geffner, J., Hermine, O., ... Amigorena, S. (2008). NADPH oxidase controls phagosomal $\mathrm{pH}$ and antigen cross-presentation in human dendritic cells. Blood. https://doi.org/10.1182/blood-2008-01-134791

Mantegazza, A. R., Zajac, A. L., Twelvetrees, A., Holzbaur, E. L. F., Amigorena, S., \& Marks, M. S. (2014). TLR-dependent phagosome tubulation in dendritic cells promotes phagosome cross-talk to optimize MHC-II antigen presentation. Proceedings of the National Academy of Sciences of the United States of America, 111(43), 15508-15513. https://doi.org/10.1073/pnas.1412998111

Marshansky, V. (2007). The V-ATPase a2-subunit as a putative endosomal pH-sensor. Biochemical Society Transactions, 35(5), 1092-1099. https://doi.org/10.1042/BST0351092

Marshansky, Vladimir, Rubinstein, J. L., \& Grüber, G. (2014). Eukaryotic V-ATPase: Novel structural findings and functional insights. Biochimica et Biophysica Acta - Bioenergetics. https://doi.org/10.1016/j.bbabio.2014.01.018

Martina, J. A., Diab, H. I., Lishu, L., Jeong-A, L., Patange, S., Raben, N., \& Puertollano, R. (2014). The nutrient-responsive transcription factor TFE3 promotes autophagy, lysosomal biogenesis, and clearance of cellular debris. Science Signaling. https://doi.org/10.1126/scisignal.2004754

Martina, J. A., \& Puertollano, R. (2017). TFEB and TFE3: The art of multi-tasking under stress conditions. Transcription, 8(1), 48-54. https://doi.org/10.1080/21541264.2016.1264353

Masiero, E., Agatea, L., Mammucari, C., Blaauw, B., Loro, E., Komatsu, M., ... Sandri, M. (2009). Autophagy Is Required to Maintain Muscle Mass. Cell Metabolism. https://doi.org/10.1016/j.cmet.2009.10.008

Masters, T. A., Pontes, B., Viasnoff, V., Li, Y., \& Gauthier, N. C. (2013). Plasma membrane 
tension orchestrates membrane trafficking, cytoskeletal remodeling, and biochemical signaling during phagocytosis. Proceedings of the National Academy of Sciences. https://doi.org/10.1073/pnas.1301766110

Masvidal, L., Hulea, L., Furic, L., Topisirovic, I., \& Larsson, O. (2017). mTOR-sensitive translation: Cleared fog reveals more trees. RNA Biology, 14(10), 1299-1305. https://doi.org/10.1080/15476286.2017.1290041

Mathaes, R., Winter, G., Besheer, A., \& Engert, J. (2014). Influence of particle geometry and PEGylation on phagocytosis of particulate carriers. International Journal of Pharmaceutics, 465(1-2), 159-164. https://doi.org/10.1016/j.ijpharm.2014.02.037

Matsuura, M. (2013). Structural Modifications of Bacterial Lipopolysaccharide that Facilitate Gram-Negative Bacteria Evasion of Host Innate Immunity. Frontiers in Immunology, 4, 109. https://doi.org/10.3389/fimmu.2013.00109

Maxson, M. E., Naj, X., O’Meara, T. R., Plumb, J. D., Cowen, L. E., \& Grinstein, S. (2018). Integrin-based diffusion barrier separates membrane domains enabling the formation of microbiostatic frustrated phagosomes. ELife, 7. https://doi.org/10.7554/eLife.34798

McCartney, A. J., Zhang, Y., \& Weisman, L. S. (2014). Phosphatidylinositol 3,5-bisphosphate: Low abundance, high significance. BioEssays, 36(1), 52-64. https://doi.org/10.1002/bies.201300012

Medina, D. L., Di Paola, S., Peluso, I., Armani, A., De Stefani, D., Venditti, R., ... Ballabio, A. (2015). Lysosomal calcium signalling regulates autophagy through calcineurin and TFEB. Nature Cell Biology, 17(3), 288-299. https://doi.org/10.1038/ncb3114

Melchionda, M., Pittman, J. K., Mayor, R., \& Patel, S. (2016). Ca2 $+/ \mathrm{H}+$ exchange by acidic organelles regulates cell migration in vivo. The Journal of Cell Biology, 212(7), 803-813. https://doi.org/10.1083/jcb.201510019

Meyuhas, O. (2000). Synthesis of the translational apparatus is regulated at the translational level. European Journal of Biochemistry, 267(21), 6321-6330.

Michelet, X., Garg, S., Wolf, B. J., Tuli, A., Ricciardi-Castagnoli, P., \& Brenner, M. B. (2015). MHC class II presentation is controlled by the lysosomal small GTPase, Arl8b. Journal of 
Immunology (Baltimore, $\quad$ Md. : 1950), $\quad$ 194(5), 2079-2088. https://doi.org/10.4049/jimmunol.1401072

Migliano, S. M., \& Teis, D. (2018). ESCRT and Membrane Protein Ubiquitination (Vol. 57, pp. 107-135). Springer International Publishing. https://doi.org/10.1007/978-3-319-96704-2_4

Mijaljica, D., Prescott, M., \& Devenish, R. J. (2011). Microautophagy in mammalian cells: revisiting a 40-year-old conundrum. Autophagy, 7(7), 673-682. https://doi.org/10.4161/auto.7.7.14733

Milde, R., Ritter, J., Tennent, G. A., Loesch, A., Martinez, F. O., Gordon, S., ... Helming, L. (2015). Multinucleated Giant Cells Are Specialized for Complement-Mediated Phagocytosis and Large Target Destruction. Cell Reports, 13(9), 1937-1948. https://doi.org/10.1016/j.celrep.2015.10.065

Millat, G., Marçais, C., Tomasetto, C., Chikh, K., Fensom, A. H., Harzer, K., .. Vanier, M. T. (2001). Niemann-Pick C1 disease: correlations between NPC1 mutations, levels of NPC1 protein, and phenotypes emphasize the functional significance of the putative sterol-sensing domain and of the cysteine-rich luminal loop. American Journal of Human Genetics, 68(6), 1373-1385. https://doi.org/10.1086/320606

Mills, J. C., \& Taghert, P. H. (2012). Scaling factors: Transcription factors regulating subcellular domains. BioEssays, 34(1), 10-16. https://doi.org/10.1002/bies.201100089

Miloslavski, R., Cohen, E., Avraham, A., Iluz, Y., Hayouka, Z., Kasir, J., .. Meyuhas, O. (2014). Oxygen sufficiency controls TOP mRNA translation via the TSC-Rheb-mTOR pathway in a 4E-BP-independent manner. Journal of Molecular Cell Biology, 6(3), 255-266. https://doi.org/10.1093/jmcb/mju008

Mohamed, M. M., \& Sloane, B. F. (2006). multifunctional enzymes in cancer. Nature Reviews Cancer, 6(10), 764-775. https://doi.org/10.1038/nrc1949

Möller, J., Luehmann, T., Hall, H., \& Vogel, V. (2012). The race to the pole: How high-aspect ratio shape and heterogeneous environments limit phagocytosis of filamentous Escherichia coli bacteria by macrophages. Nano Letters, 12(6), 2901-2905. https://doi.org/10.1021/n13004896 
Monteiro, P., Rossé, C., Castro-Castro, A., Irondelle, M., Lagoutte, E., Paul-Gilloteaux, P., ... Chavrier, P. (2013). Endosomal WASH and exocyst complexes control exocytosis of MT1MMP at invadopodia. The Journal of Cell Biology, 203(6), 1063-1079. https://doi.org/10.1083/jcb.201306162

Mony, V. K., Benjamin, S., \& O’Rourke, E. J. (2016). A lysosome-centered view of nutrient homeostasis. Autophagy, 12(4), 619-631. https://doi.org/10.1080/15548627.2016.1147671

Moon, J.-S., Hisata, S., Park, M.-A., DeNicola, G. M., Ryter, S. W., Nakahira, K., \& Choi, A. M. K. (2015). mTORC1-Induced HK1-Dependent Glycolysis Regulates NLRP3 Inflammasome Activation. Cell Reports, 12(1), 102-115. https://doi.org/10.1016/j.celrep.2015.05.046

Morita, M., Prudent, J., Basu, K., Goyon, V., Katsumura, S., Hulea, L., ... Sonenberg, N. (2017). mTOR Controls Mitochondrial Dynamics and Cell Survival via MTFP1. Molecular Cell, 67(6), 922-935.e5. https://doi.org/10.1016/j.molcel.2017.08.013

Mrakovic, A., Kay, J. G., Furuya, W., Brumell, J. H., \& Botelho, R. J. (2012). Rab7 and Arl8 GTPases are necessary for lysosome tubulation in macrophages. Traffic, 13(12), 1667-1679.

Mu, F.-T., Callaghan, J. M., Steele-Mortimer, O., Stenmark, H., Parton, R. G., Campbell, P. L., ... Toh, B.-H. (1995). EEA1, an Early Endosome-Associated Protein. Journal of Biological Chemistry, 270(22), 13503-13511. https://doi.org/10.1074/jbc.270.22.13503

Mukundan, L., Odegaard, J. I., Morel, C. R., Heredia, J. E., Mwangi, J. W., Ricardo-Gonzalez, R. R., ... Chawla, A. (2009). PPAR-delta senses and orchestrates clearance of apoptotic cells to promote tolerance. Nature Medicine, 15(11), 1266-1272. https://doi.org/10.1038/nm.2048

Mullins, C., \& Bonifacino, J. S. (2001, April). The molecular machinery for lysosome biogenesis. BioEssays. John Wiley \& Sons, Inc. https://doi.org/10.1002/bies.1048

Munson, M. J., Allen, G. F., Toth, R., Campbell, D. G., Lucocq, J. M., \& Ganley, I. G. (2015). mTOR activates the VPS34-UVRAG complex to regulate autolysosomal tubulation and cell survival. The EMBO Journal, 34(17), 2272-2290. https://doi.org/10.15252/embj.201590992

Murphy, D. B., Rath, S., Pizzo, E., Rudensky, A. Y., George, A., Larson, J. K., \& Janeway, C. A. (1992). Monoclonal antibody detection of a major self peptide. MHC class II complex. Journal of Immunology (Baltimore, Md. : 1950), 148(11), 3483-3491. 
Muta, T., \& Takeshige, K. (2001). Essential roles of CD14 and lipopolysaccharide-binding protein for activation of toll-like receptor (TLR)2 as well as TLR4: Reconstitution of TLR2- and TLR4-activation by distinguishable ligands in LPS preparations. European Journal of Biochemistry, 268(16), 4580-4589. https://doi.org/10.1046/j.1432-1327.2001.02385.x

Naik, U., \& Harrison, R. E. (2013). Phagocytosis. Colloquium Series on Building Blocks of the Cell: Cell Structure and Function, 1(3), 1-105. https://doi.org/10.4199/C00081ED1V01Y201304BBC004

Nakamura, N., Lill, J. R., Phung, Q., Jiang, Z., Bakalarski, C., de Mazière, A., ... Mellman, I. (2014). Endosomes are specialized platforms for bacterial sensing and NOD2 signalling. Nature, 509(7499), 240-244. https://doi.org/10.1038/nature13133

Nandagopal, N., \& Roux, P. P. (2015). Regulation of global and specific mRNA translation by the mTOR signaling pathway. Translation, 3(1), e983402. https://doi.org/10.4161/21690731.2014.983402

Nascimbeni, A. C., Codogno, P., \& Morel, E. (2017). Phosphatidylinositol-3-phosphate in the regulation of autophagy membrane dynamics. The FEBS Journal, 284(9), 1267-1278. https://doi.org/10.1111/febs.13987

Naufer, A., Hipolito, V. E. B., Ganesan, S., Prashar, A., Zaremberg, V., Botelho, R. J. R. J., \& Terebiznik, M. R. M. R. (2018). pH of endophagosomes controls association of their membranes with Vps34 and PtdIns(3)P levels. The Journal of Cell Biology, 217(1), 329-346. https://doi.org/10.1083/jcb.201702179

Neiss, W. F. (1984). A coat of glycoconjugates on the inner surface of the lysosomal membrane in the rat kidney. Histochemistry. https://doi.org/10.1007/BF02400979

Nie, Z., Hirsch, D. S., \& Randazzo, P. A. (2003). Arf and its many interactors. Current Opinion in Cell Biology, 15(4), 396-404. https://doi.org/10.1016/S0955-0674(03)00071-1

Nordmann, M., Cabrera, M., Perz, A., Bröcker, C., Ostrowicz, C., Engelbrecht-Vandré, S., \& Ungermann, C. (2010). The Mon1-Ccz1 complex is the GEF of the late endosomal Rab7 homolog Ypt7. Current Biology. https://doi.org/10.1016/j.cub.2010.08.002

O'Neill, L. A., \& Bowie, A. G. (2007). The family of five: TIR-domain-containing adaptors in 
Toll-like receptor signalling. Nature Reviews Immunology, 7(5), 353-364. https://doi.org/10.1038/nri2079

Odom, D. T., Dowell, R. D., Jacobsen, E. S., Gordon, W., Danford, T. W., MacIsaac, K. D., ... Fraenkel, E. (2007). Tissue-specific transcriptional regulation has diverged significantly between human and mouse. Nature Genetics. https://doi.org/10.1038/ng2047

Ohkuma, S, Moriyama, Y., \& Takano, T. (1982). Identification and characterization of a proton pump on lysosomes by fluorescein-isothiocyanate-dextran fluorescence. Proceedings of the National Academy of Sciences of the United States of America, 79(9), 2758-2762.

Ohkuma, Shoji, Moriyama, Y., \& Takano, T. (1983). Electrogenic nature of lysosomal proton pump as revealed with a cyanine dye. Journal of Biochemistry, 94(6), 1935-1943. https://doi.org/10.1093/oxfordjournals.jbchem.a134547

Oliver, C. (1983). Lysosomal heterogeneity in exocrine acinar cells. The Journal of Histochemistry and Cytochemistry: Official Journal of the Histochemistry Society, 31(1A Suppl), 222-223.

Owen, K. A., Meyer, C. B., Bouton, A. H., \& Casanova, J. E. (2014). Activation of Focal Adhesion Kinase by Salmonella Suppresses Autophagy via an Akt/mTOR Signaling Pathway and Promotes Bacterial Survival in Macrophages. PLoS Pathogens, 10(6), e1004159. https://doi.org/10.1371/journal.ppat.1004159

Ozinsky, A., Underhill, D. M., Fontenot, J. D., Hajjar, A. M., Smith, K. D., Wilson, C. B., ... Aderem, A. (2000). The repertoire for pattern recognition of pathogens by the innate immune system is defined by cooperation between toll-like receptors. Proceedings of the National Academy of Sciences of the United States of America, 97(25), 13766-13771. https://doi.org/10.1073/pnas.250476497

Palmieri, M., Impey, S., Kang, H., di Ronza, A., Pelz, C., Sardiello, M., \& Ballabio, A. (2011). Characterization of the CLEAR network reveals an integrated control of cellular clearance pathways. Human Molecular Genetics, 20(19), 3852-3866. https://doi.org/10.1093/hmg/ddr306

Pankiv, S., Alemu, E. a., Brech, A., Bruun, J.-A. A., Lamark, T., Øvervatn, A., ... Johansen, T. (2010). FYCO1 is a Rab7 effector that binds to LC3 and PI3P to mediate microtubule plus end - Directed vesicle transport. Journal of Cell Biology, 188(2), 253-269. 
https://doi.org/10.1083/jcb.200907015

Parzych, K. R., \& Klionsky, D. J. (2014). An overview of autophagy: morphology, mechanism, and regulation. Antioxidants \& Redox Signaling, 20(3), 460-473. https://doi.org/10.1089/ars.2013.5371

Pastore, N., Brady, O. A., Diab, H. I., Martina, J. A., Sun, L., Huynh, T., ... Puertollano, R. (2016). TFEB and TFE3 Cooperate in the Regulation of the Innate Immune Response in Activated Macrophages. Autophagy, (May), 00-00. https://doi.org/10.1080/15548627.2016.1179405

Pauwels, A. M., Trost, M., Beyaert, R., \& Hoffmann, E. (2017, June). Patterns, Receptors, and Signals: Regulation of Phagosome Maturation. Trends in Immunology. https://doi.org/10.1016/j.it.2017.03.006

Peterson, T. R., Laplante, M., Thoreen, C. C., Sancak, Y., Kang, S. A., Kuehl, W. M., ... Sabatini, D. M. (2009). DEPTOR Is an mTOR Inhibitor Frequently Overexpressed in Multiple Myeloma Cells and Required for Their Survival. Cell, 137(5), 873-886. https://doi.org/10.1016/j.cell.2009.03.046

Peterson, T. R., Sengupta, S. S., Harris, T. E., Carmack, A. E., Kang, S. A., Balderas, E., ... Sabatini, D. M. (2011). mTOR Complex 1 Regulates Lipin 1 Localization to Control the SREBP Pathway. Cell, 146(3), 408-420. https://doi.org/10.1016/j.cell.2011.06.034

Piehler, A. P., Grimholt, R. M., Øvstebø, R., \& Berg, J. P. (2010). Gene expression results in lipopolysaccharide-stimulated monocytes depend significantly on the choice of reference genes. BMC Immunology. https://doi.org/10.1186/1471-2172-11-21

Pitt, S. J., Funnell, T. M., Sitsapesan, M., Venturi, E., Rietdorf, K., Ruas, M., ... Sitsapesan, R. (2010). TPC2 is a novel NAADP-sensitive Ca2+ release channel, operating as a dual sensor of luminal $\mathrm{pH}$ and $\mathrm{Ca} 2+$. Journal of Biological Chemistry. https://doi.org/10.1074/jbc.M110.156927

Platt, C. D., Ma, J. K., Chalouni, C., Ebersold, M., Bou-Reslan, H., Carano, R. A. D., ... Delamarre, L. (2010). Mature dendritic cells use endocytic receptors to capture and present antigens. Proceedings of the National Academy of Sciences, 107(9), 4287-4292. https://doi.org/10.1073/pnas.0910609107 
Platt, F. M., Boland, B., \& van der Spoel, A. C. (2012). The cell biology of disease: lysosomal storage disorders: the cellular impact of lysosomal dysfunction. The Journal of Cell Biology, 199(5), 723-734. https://doi.org/10.1083/jcb.201208152

Poincloux, R., Lizárraga, F., Chavrier, P., Berghe, P. V. E. van den, Speirs, C., Dozynkiewicz, M. A., ... Norman, J. C. (2009). Matrix invasion by tumour cells: a focus on MT1-MMP trafficking to invadopodia. Journal of Cell Science, 122(Pt 17), 3015-3024. https://doi.org/10.1242/jcs.034561

Porstmann, T., Santos, C. R., Griffiths, B., Cully, M., Wu, M., Leevers, S., ... Schulze, A. (2008). SREBP Activity Is Regulated by mTORC1 and Contributes to Akt-Dependent Cell Growth. Cell Metabolism, 8(3), 224-236. https://doi.org/10.1016/j.cmet.2008.07.007

Porta, C., Riboldi, E., Ippolito, A., \& Sica, A. (2015, August). Molecular and epigenetic basis of macrophage polarized activation. Seminars in Immunology. https://doi.org/10.1016/j.smim.2015.10.003

Posch, T., Jezbera, J., Vrba, J., Šimek, K., Pernthaler, J., Andreatta, S., \& Sonntag, B. (2001). Size selective feeding in Cyclidium glaucoma (Ciliophora, Scuticociliatida) and its effects on bacterial community structure: A study from a continuous cultivation system. Microbial Ecology, 42(3), 217-227. https://doi.org/10.1007/s002480000114

Poteryaev, D., Datta, S., Ackema, K., Zerial, M., \& Spang, A. (2010). Identification of the switch in early-to-late endosome transition. Cell, 141(3), 497-508. https://doi.org/10.1016/j.cell.2010.03.011

Prashar, A., Bhatia, S., Gigliozzi, D., Martin, T., Duncan, C., Guyard, C., \& Terebiznik, M. R. (2013). Filamentous morphology of bacteria delays the timing of phagosome morphogenesis in macrophages. The Journal of Cell Biology, 203(6), 1081-1097. https://doi.org/10.1083/jcb.201304095

Prashar, A., \& Terebiznik, M. R. (2015). Legionella pneumophila: Homeward bound away from the phagosome. Current Opinion in Microbiology, 23(3), 86-93. https://doi.org/10.1016/j.mib.2014.11.008

Pu, J., Guardia, C. M., Keren-Kaplan, T., \& Bonifacino, J. S. (2016). Mechanisms and functions of lysosome positioning. Journal of Cell Science, 129(23), 4329-4339. 
https://doi.org/10.1242/jcs. 196287

Pu, J., Keren-Kaplan, T., \& Bonifacino, J. S. (2017). A Ragulator-BORC interaction controls lysosome positioning in response to amino acid availability. The Journal of Cell Biology, jcb.201703094. https://doi.org/10.1083/jcb.201703094

Pu, J., Schindler, C., Jia, R., Jarnik, M., Backlund, P., \& Bonifacino, J. S. (2015). BORC, a Multisubunit Complex that Regulates Lysosome Positioning. Developmental Cell, 33(2), 176-188. https://doi.org/10.1016/j.devcel.2015.02.011

Radtke, S., Wüller, S., Yang, X., Lippok, B. E., Mütze, B., Mais, C., ... Hermanns, H. M. (2010). Cross-regulation of cytokine signalling: pro-inflammatory cytokines restrict IL-6 signalling through receptor internalisation and degradation. Journal of Cell Science. https://doi.org/10.1242/jcs. 065326

Rambold, A. S., Kostelecky, B., Elia, N., \& Lippincott-Schwartz, J. (2011). Tubular network formation protects mitochondria from autophagosomal degradation during nutrient starvation. Proceedings of the National Academy of Sciences of the United States of America, 108(25), 10190-10195. https://doi.org/10.1073/pnas.1107402108

Rebsamen, M., Pochini, L., Stasyk, T., de Araújo, M. E. G., Galluccio, M., Kandasamy, R. K., ... Superti-Furga, G. (2015). SLC38A9 is a component of the lysosomal amino acid sensing machinery that controls mTORC1. Nature, 519(7544), 477-481. https://doi.org/10.1038/nature14107

Reczek, D., Schwake, M., Schröder, J., Hughes, H., Blanz, J., Jin, X., ... Saftig, P. (2007). LIMP2 Is a Receptor for Lysosomal Mannose-6-Phosphate-Independent Targeting of $\beta$ Glucocerebrosidase. Cell, 131(4), 770-783. https://doi.org/10.1016/J.CELL.2007.10.018

Reddy, A., Caler, E. V, \& Andrews, N. W. (2001). Plasma membrane repair is mediated by Ca(2+)regulated exocytosis of lysosomes. Cell, 106(2), 157-169. https://doi.org/10.1016/S0092$8674(01) 00421-4$

Reed, S. G., Hsu, F. C., Carter, D., \& Orr, M. T. (2016). The science of vaccine adjuvants: Advances in TLR4 ligand adjuvants. Current Opinion in Immunology. https://doi.org/10.1016/j.coi.2016.06.007 
Rigaut, G., Shevchenko, A., Rutz, B., Wilm, M., Mann, M., \& Seraphin, B. (1999). A generic protein purification method for protein complex characterization and proteome exploration. Nature Biotechnology. https://doi.org/10.1038/13732

Rink, J., Ghigo, E., Kalaidzidis, Y., \& Zerial, M. (2005). Rab conversion as a mechanism of progression from early to late endosomes. Cell, 122(5), 735-749. https://doi.org/10.1016/j.cell.2005.06.043

Robinson, J. M. (1986). Unusual lysosomes in aortic smooth muscle cells: presence in living and rapidly frozen cells. The Journal of Cell Biology, 102(5), 1615-1622. https://doi.org/10.1083/jcb.102.5.1615

Roche, P., \& Cresswell, P. (1990). Invariant chain association with HLA-DR molecules inhibits immunogenic peptide binding. Letters To Nature, 346, 183-187. https://doi.org/10.1038/346183a0

Roczniak-Ferguson, A., Petit, C. S., Froehlich, F., Qian, S., Ky, J., Angarola, B., ... Ferguson, S. M. (2012). The Transcription Factor TFEB Links mTORC1 Signaling to Transcriptional Control of Lysosome Homeostasis. Science Signaling, 5(228), ra42-ra42. https://doi.org/10.1126/scisignal.2002790

Rong, L., Livingstone, M., Sukarieh, R., Petroulakis, E., Gingras, A.-C. C., Crosby, K., ... Sonenberg, N. (2008). Control of eIF4E cellular localization by eIF4E-binding proteins, 4EBPs. $R N A, 14(7), 1318-1327$. https://doi.org/10.1261/rna.950608

Rong, Y., Liu, M., Ma, L., Du, W., Zhang, H., Tian, Y., ... Yu, L. (2012). Clathrin and phosphatidylinositol-4,5-bisphosphate regulate autophagic lysosome reformation. Nature Cell Biology, 14(9), 924-934. https://doi.org/10.1038/ncb2557

Rong, Y., Mcphee, C., Deng, S., Chen, L., Liu, M., Tracy, K., .. Marshall, J. J. (2011). Correction for Rong et al., Spinster is required for autophagic lysosome reformation and mTOR reactivation following starvation. Proceedings of the National Academy of Sciences, 108(27), 11297. https://doi.org/10.1073/pnas.1108410108

Rosa-Ferreira, C., \& Munro, S. (2011). Arl8 and SKIP Act Together to Link Lysosomes to Kinesin-1. Developmental Cell, 21(6), 1171-1178. https://doi.org/10.1016/j.devcel.2011.10.007 
Rossi, A., Deveraux, Q., Turk, B., \& Sali, A. (2004). Comprehensive search for cysteine cathepsins in the human genome. Biological Chemistry. https://doi.org/10.1515/BC.2004.040

Rostislavleva, K., Soler, N., Ohashi, Y., Zhang, L., Pardon, E., Burke, J. E., ... Williams, R. L. (2015). Structure and flexibility of the endosomal Vps34 complex reveals the basis of its function on membranes. Science, 350(6257), aac7365-aac7365. https://doi.org/10.1126/science.aac7365

Roux, P. P., \& Topisirovic, I. (2018). Signaling Pathways Involved in the Regulation of mRNA Translation. Molecular and Cellular Biology. https://doi.org/10.1128/mcb.00070-18

Sancak, Y., Bar-Peled, L., Zoncu, R., Markhard, A. L., Nada, S., \& Sabatini, D. M. (2010). Ragulator-Rag complex targets mTORC1 to the lysosomal surface and is necessary for its activation by amino acids. Cell, 141(2), 290-303. https://doi.org/10.1016/j.cell.2010.02.024

Sancak, Y., Peterson, T. R., Shaul, Y. D., Lindquist, R. A., Thoreen, C. C., Bar-Peled, L., \& Sabatini, D. M. (2008). The Rag GTPases Bind Raptor and Mediate Amino Acid Signaling to mTORC1. Science, 320(5882), 1496-1501. https://doi.org/10.1126/science.1157535

Sandri, M. (2010). Autophagy in skeletal muscle. FEBS Letters, 584(7), 1411-1416. https://doi.org/10.1016/j.febslet.2010.01.056

Sardiello, M., Palmieri, M., di Ronza, A., Medina, D. L., Valenza, M., Gennarino, V. A., ... Ballabio, A. (2009). A Gene Network Regulating Lysosomal Biogenesis and Function. Science, (July), 473-477. https://doi.org/10.1126/science.1174447

Saric, A., Hipolito, V. E. B., Kay, J. G., Canton, J., Antonescu, C. N., \& Botelho, R. J. (2016). mTOR controls lysosome tubulation and antigen presentation in macrophages and dendritic cells. Molecular Biology of the Cell, 27(2), 321-333. https://doi.org/10.1091/mbc.E15-050272

Sasaki, T, Kikuchi, A., Araki, S., Hata, Y., Isomura, M., Kuroda, S., \& Takai, Y. (1990). Purification and characterization from bovine brain cytosol of a protein that inhibits the dissociation of GDP from and the subsequent binding of GTP to smg p25A, a ras p21-like GTP-binding protein. The Journal of Biological Chemistry, 265(4), 2333-2337.

Sasaki, Takehiko, Takasuga, S., Sasaki, J., Kofuji, S., Eguchi, S., Yamazaki, M., \& Suzuki, A. 
(2009). Mammalian phosphoinositide kinases and phosphatases. Progress in Lipid Research, 48(6), 307-343. https://doi.org/10.1016/j.plipres.2009.06.001

Savina, A., \& Amigorena, S. (2007, October). Phagocytosis and antigen presentation in dendritic cells. Immunological Reviews. https://doi.org/10.1111/j.1600-065X.2007.00552.x

Savina, A., Jancic, C., Hugues, S., Guermonprez, P., Vargas, P., Moura, I. C., ... Amigorena, S. (2006). NOX2 controls phagosomal $\mathrm{pH}$ to regulate antigen processing during crosspresentation by dendritic cells. Cell, 126(1), 205-218. https://doi.org/10.1016/j.cell.2006.05.035

Scheffzek, K., Ahmadian, M. R., Kabsch, W., Wiesmüller, L., Lautwein, A., Schmitz, F., \& Wittinghofer, A. (1997). The Ras-RasGAP complex: structural basis for GTPase activation and its loss in oncogenic Ras mutants. Science (New York, N.Y.), 277(5324), 333-338.

Schink, K. O., Raiborg, C., \& Stenmark, H. (2013). Phosphatidylinositol 3-phosphate, a lipid that regulates membrane dynamics, protein sorting and cell signalling. BioEssays, n/a-n/a. https://doi.org/10.1002/bies.201300064

Schmid, A. C., Wise, H. M., Mitchell, C. A., Nussbaum, R., \& Woscholski, R. (2004). Type II phosphoinositide 5-phosphatases have unique sensitivities towards fatty acid composition and head group phosphorylation. FEBS Letters, 576(1-2), 9-13. https://doi.org/10.1016/j.febslet.2004.08.052

Schott, J., Reitter, S., Philipp, J., Haneke, K., Schäfer, H., \& Stoecklin, G. (2014). Translational Regulation of Specific mRNAs Controls Feedback Inhibition and Survival during Macrophage Activation. PLoS Genetics. https://doi.org/10.1371/journal.pgen.1004368

Schröder, B. A., Wrocklage, C., Hasilik, A., \& Saftig, P. (2010). The proteome of lysosomes. Proteomics. https://doi.org/10.1002/pmic.201000196

Schwarz, D. S., \& Blower, M. D. (2016). The endoplasmic reticulum: structure, function and response to cellular signaling. Cellular and Molecular Life Sciences : CMLS, 73(1), 79-94. https://doi.org/10.1007/s00018-015-2052-6

Semenza, G L. (2010). Defining the role of hypoxia-inducible factor 1 in cancer biology and therapeutics. Oncogene, 29(5), 625-634. https://doi.org/10.1038/onc.2009.441 
Semenza, Gregg L, Nejfelt, M. K., Chi, S. M., \& Antonarakis, S. E. (1991). Hypoxia-inducible nuclear factors bind to an enhancer element located 3' to the human erythropoietin gene. Proceedings of the National Academy of Sciences of the United States of America, 88(13), $5680-5684$.

Seto, E. S., Bellen, H. J., \& Lloyd, T. E. (2002). When cell biology meets development: Endocytic regulation of signaling pathways. Genes and Development, 16(713), 1314-1336. https://doi.org/10.1101/gad.989602

Settembre, C., \& Ballabio, A. (2014). Lysosomal Adaptation: How the Lysosome Responds to External Cues. Cold Spring Harbor Perspectives in Biology, 6(6), a016907-a016907. https://doi.org/10.1101/cshperspect.a016907

Settembre, C., Di Malta, C., Polito, V. A., Arencibia, M. G., Vetrini, F., Erdin, S., ... Ballabio, A. (2011). TFEB Links Autophagy to Lysosomal Biogenesis. Science, 332(6036), 1429-1433. https://doi.org/10.1126/science.1204592

Settembre, C., Zoncu, R., Medina, D. L., Vetrini, F., Erdin, S., Erdin, S., ... Ballabio, A. (2012). A lysosome-to-nucleus signalling mechanism senses and regulates the lysosome via mTOR and TFEB. The EMBO Journal, 31(5), 1095-1108. https://doi.org/10.1038/emboj.2012.32

Shahbazian, D., Roux, P. P., Mieulet, V., Cohen, M. S., Raught, B., Taunton, J., ... Sonenberg, N. (2006). The mTOR/PI3K and MAPK pathways converge on eIF4B to control its phosphorylation and activity. The EMBO Journal, 25(12), 2781-2791. https://doi.org/10.1038/sj.emboj.7601166

Shimazu, R., Akashi, S., Ogata, H., Nagai, Y., Fukudome, K., Miyake, K., \& Kimoto, M. (1999). MD-2, a molecule that confers lipopolysaccharide responsiveness on Toll-like receptor 4. The Journal of Experimental Medicine, 189(11), 1777-1782. https://doi.org/10.1084/jem.189.11.1777

Shorter, J. (2016). Phasing in and out. Nature Chemistry, 8(6), 528-530. https://doi.org/10.1038/nchem.2534

Shulga, Y. V., Anderson, R. A., Topham, M. K., \& Epand, R. M. (2012). Phosphatidylinositol-4phosphate 5-Kinase Isoforms Exhibit Acyl Chain Selectivity for Both Substrate and Lipid Activator. Journal of Biological Chemistry, 287(43), 35953-35963. 
https://doi.org/10.1074/jbc.M112.370155

Smith, G. A., Howell, G. J., Phillips, C., Muench, S. P., Ponnambalam, S., \& Harrison, M. A. (2016). Extracellular and Luminal $\mathrm{pH}$ Regulation by Vacuolar H+-ATPase Isoform Expression and Targeting to the Plasma Membrane and Endosomes. The Journal of Biological Chemistry, 291(16), 8500-8515. https://doi.org/10.1074/jbc.M116.723395

Son, S. M., Park, S. J., Lee, H., Siddiqi, F., Lee, J. E., Menzies, F. M., \& Rubinsztein, D. C. (2019). Leucine Signals to mTORC1 via Its Metabolite Acetyl-Coenzyme A. Cell Metabolism. https://doi.org/10.1016/j.cmet.2018.08.013

Steinman, R M, \& Swanson, J. (1995). The endocytic activity of dendritic cells. The Journal of Experimental Medicine, 182(2), 283-288.

Steinman, Ralph M., Brodie, S. E., \& Cohn, Z. A. (1976). Membrane flow during pinocytosis: A stereologic analysis. Journal of Cell Biology. https://doi.org/10.1083/jcb.68.3.665

Stephen, T. L., Fabri, M., Groneck, L., Röhn, T. A., Hafke, H., Robinson, N., ... Kalka-Moll, W. M. (2007). Transport of Streptococcus pneumoniae capsular polysaccharide in MHC Class II tubules. PLoS Pathogens, 3(3), e32. https://doi.org/10.1371/journal.ppat.0030032

Stjepanovic, G., Baskaran, S., Lin, M. G., \& Hurley, J. H. (2017). Vps34 Kinase Domain Dynamics Regulate the Autophagic PI 3-Kinase Complex. Molecular Cell, 67(3), 528534.e3. https://doi.org/10.1016/j.molcel.2017.07.003

Stoddard, A., \& Rolland, V. (2019). I see the light! Fluorescent proteins suitable for cell wall/apoplast targeting in Nicotiana benthamiana leaves. Plant Direct. https://doi.org/10.1002/pld3.112

Stumptner, P., \& Benaroch, P. (1997). Interaction of MHC class II molecules with the invariant chain: role of the invariant chain (81-90) region. The EMBO Journal, 16(19), 5807-5818. https://doi.org/10.1038/sj.emboj.7590555

Su, H., Yang, F., Wang, Q., Shen, Q., Huang, J., Peng, C., .. Liu, W. (2017). VPS34 Acetylation Controls Its Lipid Kinase Activity and the Initiation of Canonical and Non-canonical Autophagy. Molecular Cell, 676), 907-921.e7. https://doi.org/10.1016/J.MOLCEL.2017.07.024 
Sun-Wada, G.-H., Tabata, H., Kawamura, N., Aoyama, M., \& Wada, Y. (2009). Direct recruitment of H+-ATPase from lysosomes for phagosomal acidification. J Cell Sci, 122(14), 2504-2513. https://doi.org/10.1242/JCS.050443

Svitkin, Y. V, Pause, A., Haghighat, A., Pyronnet, S., Witherell, G., Belsham, G. J., \& Sonenberg, N. (2001). The requirement for eukaryotic initiation factor 4A (elF4A) in translation is in direct proportion to the degree of mRNA 5' secondary structure. RNA (New York, N.Y.), 7(3), $382-394$.

Swanson, J. A., Yirinec, B. D., \& Silverstein, S. C. (1985). Phorbol esters and horseradish peroxidase stimulate pinocytosis and redirect the flow of pinocytosed fluid in macrophages. Journal of Cell Biology, 100(3), 851-859. https://doi.org/10.1083/jcb.100.3.851

Swanson, J., Burke, E., \& Silverstein, S. C. (1987). Tubular lysosomes accompany stimulated pinocytosis in macrophages. Journal of Cell Biology, 104(5), 1217-1222. https://doi.org/10.1083/jcb.104.5.1217

Swanson, J a, Johnson, M. T., Beningo, K., Post, P., Mooseker, M., \& Araki, N. (1999). A contractile activity that closes phagosomes in macrophages. Journal of Cell Science, 112 ( Pt $3,307-316$.

Swanson, Joel, Bushnell, A., \& Silverstein, S. C. (1987). Tubular lysosome morphology and distribution within macrophages depend on the integrity of cytoplasmic microtubules. Proceedings of the National Academy of Sciences, 84(7), 1921-1925. https://doi.org/10.1073/pnas.84.7.1921

ten Broeke, T., Wubbolts, R., \& Stoorvogel, W. (2013). MHC Class II Antigen Presentation by Dendritic Cells Regulated through Endosomal Sorting. Cold Spring Harbor Perspectives in Biology, 5(12), a016873-a016873. https://doi.org/10.1101/cshperspect.a016873

Thoreen, C. C. (2017). The molecular basis of mTORC1-regulated translation. Biochemical Society Transactions, 45(1), 213-221. https://doi.org/10.1042/BST20160072

Thoreen, C. C., Chantranupong, L., Keys, H. R., Wang, T., Gray, N. S., \& Sabatini, D. M. (2012). A unifying model for mTORC1-mediated regulation of mRNA translation. Nature, 485(7396), 109-113. https://doi.org/10.1038/nature11083 
Tian, W., Li, X. J., Stull, N. D., Ming, W., Suh, C.-I., Bissonnette, S. A., .. Dinauer, M. C. (2008).

Fc R-stimulated activation of the NADPH oxidase: phosphoinositide-binding protein p40phox regulates NADPH oxidase activity after enzyme assembly on the phagosome. Blood, 112(9), 3867-3877. https://doi.org/10.1182/blood-2007-11-126029

Tomasevic, N., Jia, Z., Russell, A., Fujii, T., Hartman, J. J., Clancy, S., ... Sakowicz, R. (2007). Differential regulation of WASP and N-WASP by Cdc42, Rac1, Nck, and PI(4,5)P2. Biochemistry, 46(11), 3494-3502. https://doi.org/10.1021/bi062152y

Trombetta, E. S., Ebersold, M., Garrett, W., Pypaert, M., \& Mellman, I. (2003). Activation of lysosomal function during dendritic cell maturation. Science, 299(5611), 1400-1403. https://doi.org/10.1126/science.1080106

Tuli, A., Thiery, J., James, A. M., Michelet, X., Sharma, M., Garg, S., .. Brenner, M. B. (2013). Arf-like GTPase Arl8b regulates lytic granule polarization and natural killer cell-mediated cytotoxicity. Molecular Biology of the Cell, 24(23), 3721-3735. https://doi.org/10.1091/mbc.e13-05-0259

Valvezan, A. J., \& Manning, B. D. (2019). Molecular logic of mTORC1 signalling as a metabolic rheostat. Nature Metabolism, 1(3), 321-333. https://doi.org/10.1038/s42255-019-0038-7

Vellodi, A. (2005). Lysosomal storage disorders. British Journal of Haematology, 128(4), 413431. https://doi.org/10.1111/j.1365-2141.2004.05293.x

Verbsky, J. W., \& Chatila, T. A. (2014). Immune Dysregulation Leading to Chronic Autoimmunity. In Stiehm's Immune Deficiencies (pp. 497-516). Elsevier. https://doi.org/10.1016/B978-0-12-405546-9.00023-6

Vieira, O. V., Botelho, R. J., Rameh, L., Brachmann, S. M., Matsuo, T., Davidson, H. W., ... Grinstein, S. (2001). Distinct roles of class I and class III phosphatidylinositol 3-kinases in phagosome formation and maturation. The Journal of Cell Biology, 155(1), 19-26. https://doi.org/10.1083/jcb.200107069

Vieira, O. V, Botelho, R. J., \& Grinstein, S. (2002). Phagosome maturation: aging gracefully. The Biochemical Journal, 366(Pt 3), 689-704. https://doi.org/10.1042/BJ20020691

Vyas, J. M., Kim, Y.-M., Artavanis-Tsakonas, K., Love, J. C., Van der Veen, A. G., \& Ploegh, H. 
L. (2007). Tubulation of class II MHC compartments is microtubule dependent and involves multiple endolysosomal membrane proteins in primary dendritic cells. Journal of Immunology (Baltimore, $\quad$ Md. : 1950$), \quad$ 178(11), $\quad$ 7199-7210. https://doi.org/10.4049/jimmunol.178.11.7199

Wang, C., Du, W., Su, Q. P., Zhu, M., Feng, P., Li, Y., .. Yu, L. (2015). Dynamic tubulation of mitochondria drives mitochondrial network formation. Cell Research, 25(10), 1108-1120. https://doi.org/10.1038/cr.2015.89

Wang, L., Harris, T. E., Roth, R. A., \& Lawrence, J. C. (2007). PRAS40 Regulates mTORC1 Kinase Activity by Functioning as a Direct Inhibitor of Substrate Binding. Journal of Biological Chemistry, 282(27), 20036-20044. https://doi.org/10.1074/jbc.M702376200

Wang, S., Tsun, Z.-Y., Wolfson, R. L., Shen, K., Wyant, G. A., Plovanich, M. E., ... Sabatini, D. M. (2015). Lysosomal amino acid transporter SLC38A9 signals arginine sufficiency to mTORC1. Science, 347(6218), 188-194. https://doi.org/10.1126/science.1257132

Wang, X., Li, W., Williams, M., Terada, N., Alessi, D. R., \& Proud, C. G. (2001). Regulation of elongation factor 2 kinase by p90(RSK1) and p70 S6 kinase. The EMBO Journal, 20(16), 4370-4379. https://doi.org/10.1093/emboj/20.16.4370

Watts, C. (1997). Capture and processing of exogenous antigens for presentation on MHC molecules. Annual Review of Immunology, 15, 821-850. https://doi.org/10.1146/annurev.immunol.15.1.821

Weischenfeldt, J., \& Porse, B. (2008). Bone Marrow-Derived Macrophages (BMM): Isolation and Applications. Cold Spring Harbor Protocols, 2008(12), pdb.prot5080-pdb.prot5080. https://doi.org/10.1101/pdb.prot5080

Wildschutte, H., Wolfe, D. M., Tamewitz, A., \& Lawrence, J. G. (2004). Protozoan predation, diversifying selection, and the evolution of antigenic diversity in Salmonella. Proceedings of the National Academy of Sciences, 101(29), 10644-10649. https://doi.org/10.1073/pnas.0404028101

Wittinghofer, A. (2014). Ras Superfamily Small G Proteins: Biology and Mechanisms 2. (A. Wittinghofer, Ed.), Transport. Cham: Springer International Publishing. https://doi.org/10.1007/978-3-319-07761-1 
Wolfson, R. L., Chantranupong, L., Saxton, R. A., Shen, K., Scaria, S. M., Cantor, J. R., \& Sabatini, D. M. (2016). Sestrin2 is a leucine sensor for the mTORC1 pathway. Science, 351(6268), 43-48. https://doi.org/10.1126/science.aab2674

Xiong, J., \& Zhu, M. X. (2016). Regulation of lysosomal ion homeostasis by channels and transporters. Science China Life Sciences, 59(8), 777-791. https://doi.org/10.1007/s11427016-5090-x

Xu, H., \& Ren, D. (2015). Lysosomal Physiology. Annual Review of Physiology, 77(1), 57-80. https://doi.org/10.1146/annurev-physiol-021014-071649

Yamaguchi, S., Jha, A., Li, Q., Soyombo, A. A., Dickinson, G. D., Churamani, D., ... Muallem, S. (2011). Transient receptor potential mucolipin 1 (TRPML1) and two-pore channels are functionally independent organellar ion channels. The Journal of Biological Chemistry, 286(26), 22934-22942. https://doi.org/10.1074/jbc.M110.210930

Yamashita, R., Suzuki, Y., Takeuchi, N., Wakaguri, H., Ueda, T., Sugano, S., \& Nakai, K. (2008). Comprehensive detection of human terminal oligo-pyrimidine (TOP) genes and analysis of their characteristics. Nucleic Acids Research, 36(11), 3707-3715. https://doi.org/10.1093/nar/gkn248

Yang, D. C., Blair, K. M., \& Salama, N. R. (2016). Staying in Shape: the Impact of Cell Shape on Bacterial Survival in Diverse Environments. Microbiology and Molecular Biology Reviews, 80(1), 187-203. https://doi.org/10.1128/MMBR.00031-15

Yu, Le, Wu, W. K. K., Gu, C., Zhong, D., Zhao, X., Kong, Y., ... Liu, S. (2016). Obatoclax impairs lysosomal function to block autophagy in cisplatin-sensitive and -resistant esophageal cancer cells. Oncotarget, 7(12), 14693-14707. https://doi.org/10.18632/oncotarget.7492

Yu, Li, McPhee, C. K., Zheng, L., Mardones, G. a, Rong, Y., Peng, J., ... Lenardo, M. J. (2010). Termination of autophagy and reformation of lysosomes regulated by mTOR. Nature, 465(June), 942-946. https://doi.org/10.1038/nature09076

Yuan, S., Li, J., Diener, D. R., Choma, M. A., Rosenbaum, J. L., \& Sun, Z. (2012). Target-ofrapamycin complex 1 (Torc1) signaling modulates cilia size and function through protein synthesis regulation. Proceedings of the National Academy of Sciences of the United States of America, 109(6), 2021-2026. https://doi.org/10.1073/pnas.1112834109 
Zent, C. S., \& Elliott, M. R. (2017). Maxed out macs: physiologic cell clearance as a function of macrophage phagocytic capacity. The FEBS Journal, 284(7), 1021-1039. https://doi.org/10.1111/febs.13961

Zhang, C. S., Jiang, B., Li, M., Zhu, M., Peng, Y., Zhang, Y. L., ... Lin, S. C. (2014). The lysosomal v-ATPase-ragulator complex is a common activator for AMPK and mTORC1, acting as a switch between catabolism and anabolism. Cell Metabolism, 20(3), 526-540. https://doi.org/10.1016/j.cmet.2014.06.014

Zhen, Y., \& Stenmark, H. (2015). Cellular functions of Rab GTPases at a glance. Journal of Cell Science, 128(17), 3171-3176. https://doi.org/10.1242/jcs.166074

Zhu, M. X., Evans, A. M., Ma, J., Parrington, J., \& Galione, A. (2010). Two-pore channels for integrative $\mathrm{Ca} 2+$ signaling. Communicative and Integrative Biology. https://doi.org/10.4161/cib.3.1.9864

Zoncu, R., Bar-Peled, L., Efeyan, A., Wang, S., Sancak, Y., \& Sabatini, D. M. (2011). mTORC1 senses lysosomal amino acids through an inside-out mechanism that requires the vacuolar H+-ATPase. Science, 334(6056), 678-683. https://doi.org/10.1126/science.1207056

Zoncu, R., Efeyan, A., \& Sabatini, D. M. (2011). MTOR: From growth signal integration to cancer, diabetes and ageing. Nature Reviews Molecular Cell Biology. https://doi.org/10.1038/nrm3025 